Dietrich Kettler, Alfred Simon, Reiner Anselm, Volker Lipp, Gunnar Duttge (Hg.)

\title{
Selbstbestimmung am Lebensende
}

Ringvorlesung im Wintersemester 2005/06

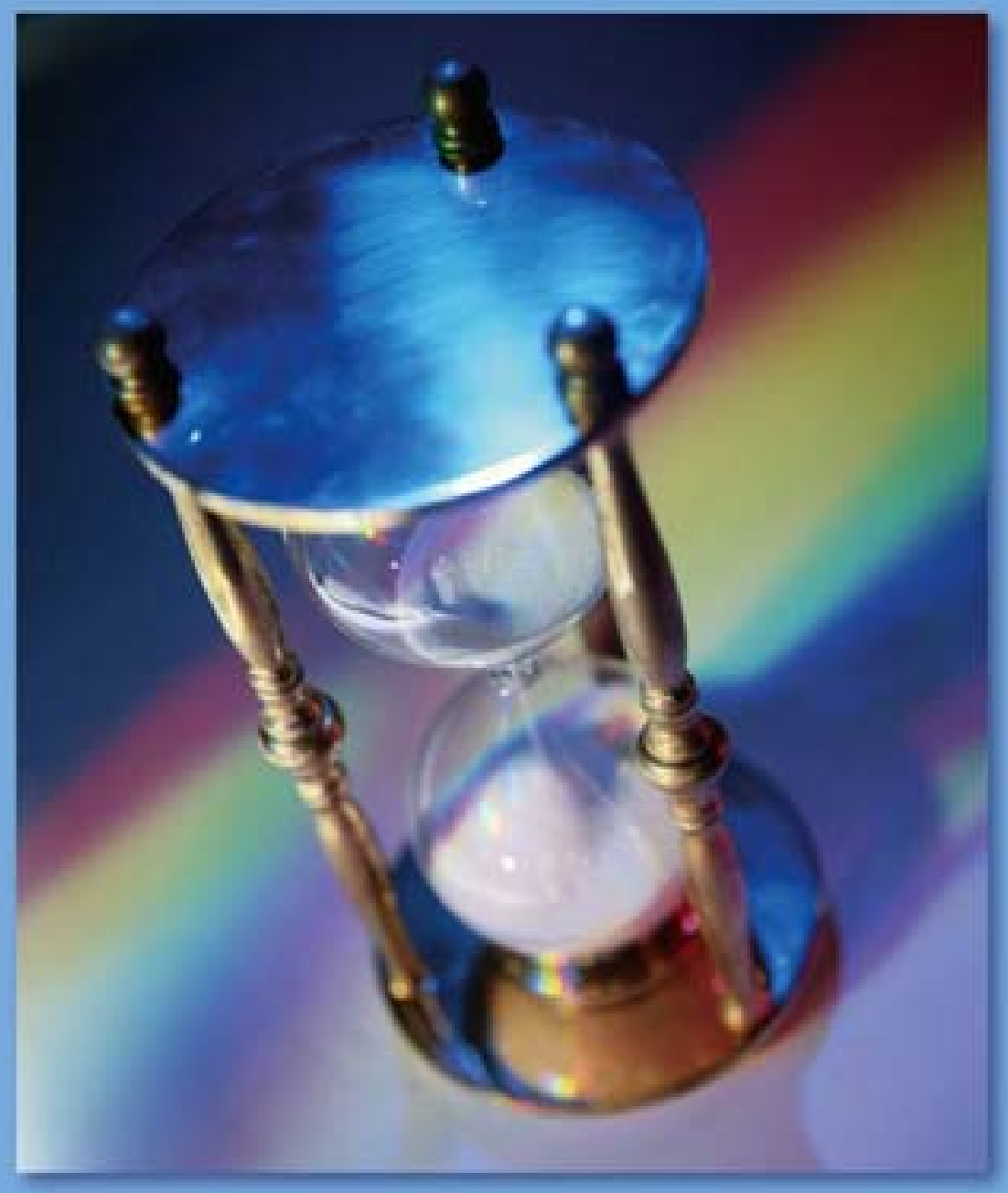

\section{A Universitätsverlag Göttingen}



Dietrich Kettler, Alfred Simon, Reiner Anselm, Volker Lipp und Gunnar Duttge (Hg.)

Selbstbestimmung am Lebensende 
erschienen

im Universitätsverlag Göttingen 2006 
Dietrich Kettler, Alfred Simon,

Reiner Anselm, Volker Lipp und

Gunnar Duttge (Hg.)

Selbstbestimmung am

Lebensende

Ringvorlesung im Wintersemester 2005/06

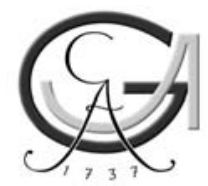

Universitätsverlag Göttingen 2006 


\section{Bibliographische Information der Deutschen Nationalbibliothek}

Die Deutsche Nationalbibliothek verzeichnet diese Publikation in der Deutschen Nationalbibliographie; detaillierte bibliographische Daten sind im Internet über $<$ http://dnb.ddb.de> abrufbar

Die Herausgeber:

Prof.(em.) Dr. med. Dr. h.c. Dietrich Kettler, Medizinische Fakultät

Dr. phil. Alfred Simon, Akademie für Ethik in der Medizin e. V.

Prof. Dr. theol. Reiner Anselm, Theologische Fakultät

Prof. Dr. jur. Volker Lipp, Juristische Fakultät

Prof. Dr. jur. Gunnar Duttge, Juristische Fakultät

Georg-August-Universität Göttingen

http://www.uni-goettingen.de

Dieses Buch ist auch als freie Onlineversion über die Homepage des Verlags sowie über den OPAC der Niedersächsischen Staats- und Universitätsbibliothek (http://www.sub.uni-goettingen.de) erreichbar und darf gelesen, heruntergeladen sowie als Privatkopie ausgedruckt werden [Es gelten die Lizenzbestimmungen der Onlineversion]. Es ist nicht gestattet, Kopien oder gedruckte Fassungen der freien Onlineversion zu veräußern.

Satz und Layout: Andrea Bojarra

Titelabbildung: Sanduhr, Hintergrund des Plakats zur gleichnamingen Ringvorlesung im Wintersemester 2005/06

Umschlaggestaltung: Kilian Klapp, Maren Büttner

(C) 2006 Universitätsverlag Göttingen

http://univerlag.uni-goettingen.de

ISBN-10: 3-938616-65-2

ISBN-13: 978-3-938616-65-9 


\section{Inhaltsverzeichnis}

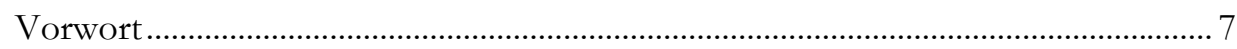

Sterben in Deutschland (Hannes Friedrich) .............................................................. 9

Rechtliche Typenbildung: Aktive und passive, direkte und indirekte Sterbehilfe

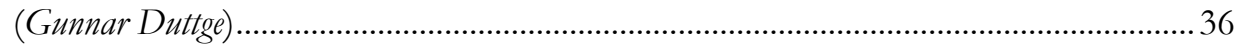

Selbstbestimmung bei Wachkoma und Demenz (Hilmar Prange)................................ 69

Rechtliche Grundlagen der Entscheidung über den Einsatz lebenserhaltender

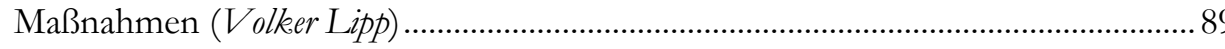

Die Praxis der aktiven Sterbehilfe in den Niederlanden und Belgien (Alfred Simon).

„Terminale Sedierung“ - Ausweg im Einzelfall, Mittelweg oder schiefe Ebene? (Christof Müller-Busch)

Menschenwürdig sterben. Die Diskussion um die Sterbehilfe aus der Sicht der evangelischen Kirche und der evangelischen Ethik (Reiner Anselm)

Sterben wo und wie? Palliativmedizin - die vierte Dimensionen in der modernen Gesundheitsversorgung (Dietrich Kettler) 150

Autorenverzeichnis. 161 



\section{Vorwort}

Ärztliches und pflegerisches Handeln war über viele Jahrhunderte hinweg geprägt durch das Primat der Fürsorge. Respekt vor der Autonomie spielte demgegenüber eine oft untergeordnete Rolle. In traditionellen ärztlichen und pflegerischen Kodizes wie dem hippokratischen Eid (ca. 400 v. Chr.), dem Eid von Florence Nightingale (1820-1910), aber auch dem Genfer Ärztegelöbnis von 1949, das die Präambel der (Muster-)Berufsordnung der deutschen Ärzteschaft bildet, fehlt jeglicher Hinweis auf den Willen des Patienten.

Das hat sich in den letzten Jahrzehnten stark verändert. Eng verbunden mit dem gesellschaftlichen Wertewandel hin zu mehr Individualität und Eigenverantwortlichkeit hat das Selbstbestimmungsrecht des Patienten deutlich an Bedeutung gewonnen. Aktuelle Entscheidungen des Bundesgerichtshofs sowie die Grundsätze der Bundesärztekammer zur ärztlichen Sterbebegleitung von 1998 und 2004 betonen den Vorrang des Patientenwillens gegenüber dem, was Ärzte und Pflegende als das Wohl des Patienten ansehen. Rechtsinstrumente wie die Patientenverfügung ermöglichen es dem (künftigen) Patienten, sein Selbstbestimmungsrecht auch in Situationen wahrzunehmen, in denen er selbst nicht mehr kommunikations- und entscheidungsfähig ist.

Mit den medizinischen, ethischen und rechtlichen Auswirkungen dieser Entwicklung auf Entscheidungen am Ende des menschlichen Lebens beschäftigte sich die Ringvorlesung „Selbstbestimmung am Lebensende“ im Wintersemester 2005/06 an der Georg-August-Universität Göttingen. Veranstalter waren die Medizinische Fakultät, die Juristische Fakultät, die Theologische Fakultät sowie die Akademie für Ethik in Medizin e. V.

Der vorliegende Sammelband enthält die für die Publikation überarbeiteten und zum Teil erweiterten Beiträge der Ringvorlesung, die damit einem breiteren Publikum verfügbar gemacht werden.

Unser besonderer Dank gilt Frau Andrea Bojarra, M.A., für ihre redaktionelle Mitarbeit bei der Erstellung des Sammelbandes.

Göttingen, im Oktober 2006

Die Herausgeber 



\section{Sterben in Deutschland}

\section{Hannes Friedrich}

Wenn man verschiedene Personen unterschiedlicher Altersgruppen bittet, ihr bisheriges Leben von Geburt an bis heute grafisch darzustellen, dann erhält man meistens relativ einförmig folgende Kurve (Abb. 1):

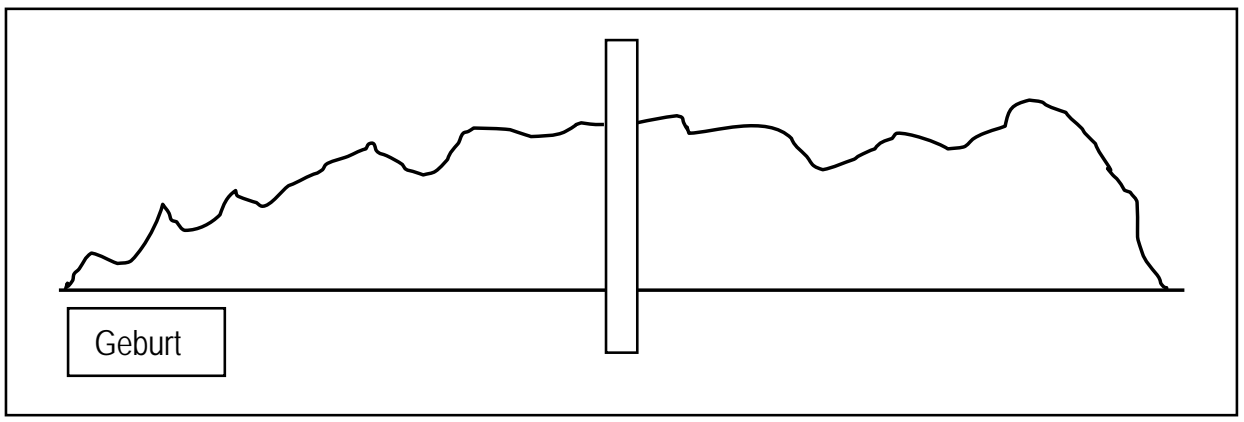

Abb. 1: Biografiekurve 
Wenn man sie dann bittet, ihr weiteres Leben zu projizieren, geht es meistens in derselben Weise weiter, um dann ganz plötzlich zum Schluss nach unten abzustürzen. In der Regel sind diese projektiven, durchschnittlich zu erwartenden Biografiekurven angelehnt an die üblichen Erwartungsnormen psychischer und sozialer Entwicklung, die meistens nach der Regel verlaufen: durch Krisen wachsen wir, die Entwicklung geht stetig positiv - trotz aller Widrigkeiten - nach oben, um sich dann auf einem bestimmten Niveau zu stabilisieren, die biografischen Lebensphasen enthalten entsprechende Determinanten: Kindheit/Jugend, junges Erwachsensein mit Eheschließung und Familiengründung, Familienphase mit schulpflichtigen Kindern, Familiephase mit erwachsenen, die Familie verlassenden Kindern, die Ruhestandsphase und neuerdings - als neueste Etappe - neben dem dritten Alter - das sog. „,vierte Alter“, das sich inzwischen bis über das 80ste Lebensjahr hinweg erweitert hat. In diesen Entwürfen sind meistens nur Akutkrankheiten angedacht, wie wir es meist aus der eigenen Entwicklung als Kinder und Jugendliche und junge Erwachsene kennen, Krankheiten, die kommen und gehen, bewältigt werden können und verschwinden. Selten wird daran gedacht, dass es im Leben Krankheiten gibt, die akut sind, aber lebensgefährlich, die kommen und nicht wieder gehen, im Sinne von chronischen Krankheiten, und vor allem denken die wenigsten daran, dass nach dem 45 . Lebensjahr die häufigsten chronischen Krankheiten ausbrechen, die in ihrer Mehrheit zumeist auch den Anfang des Verfalls enthalten in dem Sinne, dass bei vielen chronischen und degenerativen Erkrankungen auch Sterbemöglichkeiten in den späteren Lebensphasen enthalten sind. Wenn man daran denkt, dass in der Medizin mittlerweile über 50.000 Krankheiten bekannt sind, kann man sich gut vorstellen, in welchem Ausmaße wir als Objekte der Natur, der Umwelt und unserer Psyche der Gefahr von Krankheiten ausgesetzt sind, die uns nicht nur in unseren Möglichkeiten einschränken und beeinträchtigen, sondern die auch schnell, langsam, schleichend, manifest oder latent zum Verlust von Gesundheit, Leistungskraft, Lebenserwartungen und zum Sterben und Tod führen können. Aber diese Möglichkeiten werden von den meisten Menschen in Bezug auf die Betrachtung ihres eigenen Lebens lange ausgeblendet und geleugnet.

Diese Art der Ausblendung hat auch etwas mit meinem Thema „Sterben in Deutschland“ zu tun. Betrachtet man sich die vorliegenden Daten, Erkenntnisse und empirischen Forschungen über Sterbewirklichkeiten in Deutschland, so kann man feststellen, dass es in bezog Bezug auf die in der gesellschaftlichen Wirklichkeit vorkommenden Vorgänge des Sterbens und des Todes sehr wenige empirische Untersuchungen und Ergebnisse gibt. Es gibt eine Fülle von Veröffentlichungen, Film- und Fernsehbeiträgen über ausgewählte Bereiche des Sterbens und des Todes, geradezu massenhaft sind die Beiträge über Palliativmedizin und pflege, Hospizversorgung, aktive und passive Sterbehilfe angewachsen, es gibt eine ganze Reihe von Studien über Leben und Sterben durch Krebs, während beispielsweise Sterben und Tod durch andere Krankheiten relativ wenig doku- 
mentiert und untersucht worden sind. Zur allgemeinen Wirklichkeit des Sterbens außerhalb von Hospizen und Palliativstationen und ausgewählten Krebsstationen gibt es stattdessen viele Schlagworte, Zerrbilder, ängstigende Vorurteile über menschenunwürdige Medizin, Auslieferung an die medizinische Technologie, abgeschoben und isoliert im Krankenhaus sterben, Depersonalisierung und Dehumanisierung von Sterbenden und von multimorbiden alten Patienten, vom Siechtum in Alten- und Pflegeheimen. Aber Statistiken, epidemiologische Befunde, empirische Untersuchungen sind eher selten. Ich will versuchen, einige Aspekte in meinem Vortrag in Bezug auf Sterbewirklichkeiten in Deutschland zu beleuchten.

Der Tod kommt für die wenigsten Menschen plötzlich: $95 \%$ der Menschen sterben an Krankheiten, die schon länger bestehen. Für sie gibt es eine Zeit des Sterbens. Tage, Wochen, Monate und sogar Jahre.

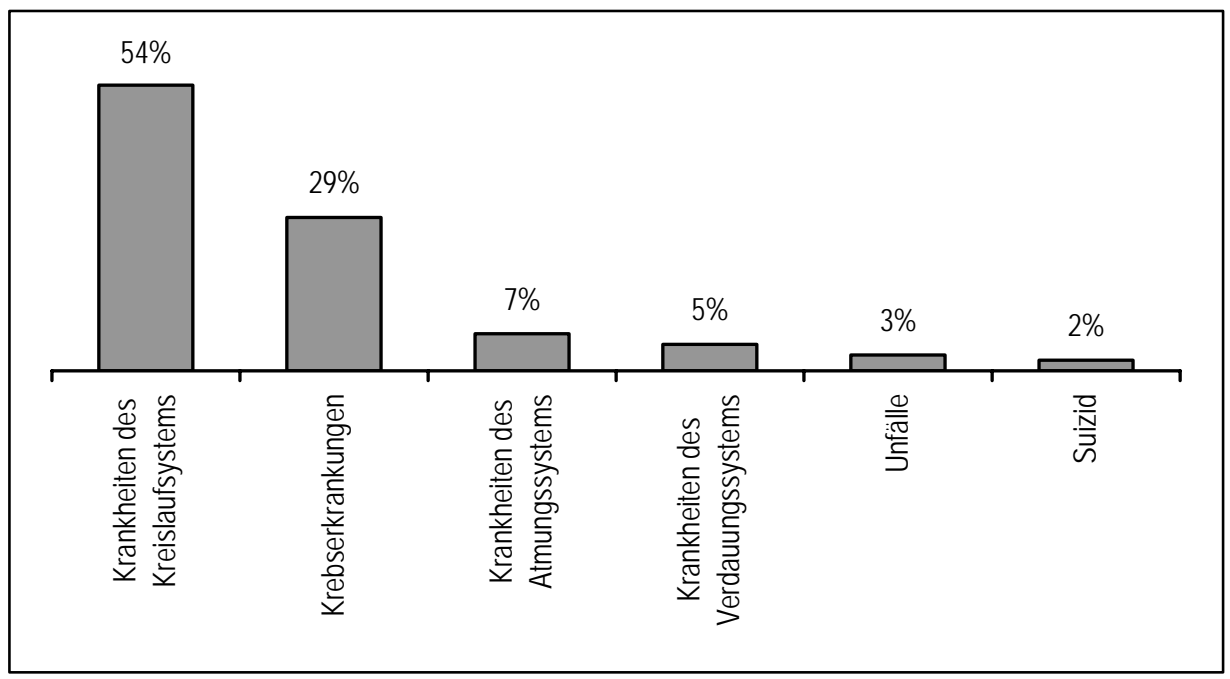

Abb. 2: Todesursachen in Deutschland (Quelle: Statistisches Bundesamt Wiesbaden, 2001)

Befragt man die Menschen, wie sie sich mit dem eigenen Sterben auseinandersetzen und das Thema bei sich zulassen, kommt ein typisches Bild zum Zug (vgl. Abb. 3): $60 \%$ wünschen sich einen schnellen und plötzlichen Tod, $25 \%$ haben sich bisher keine Gedanken darüber gemacht. Der Schluss liegt nahe: Die meisten Menschen sind nicht bereit, sich realititätsbezogen mit ihrem eigenen Sterben und Tod auseinanderzusetzen, eine Diskrepanz zwischen Wunsch und Wirklichkeit des Sterbens besteht. 


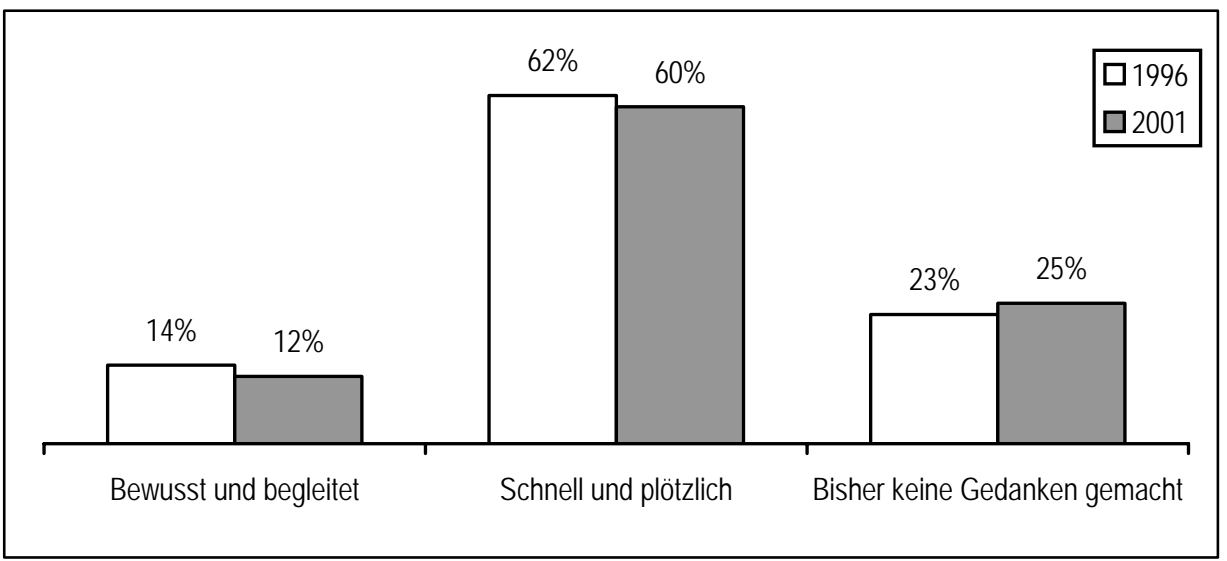

Abb. 3: Wie möchten Sie sterben? (Quelle: Deutsche Hospizstiftung, Emnid-Umfrage 2001)

Geschlechtsspezifisch gibt es Unterschiede (vgl. Abb. 4): Frauen sind eher bereit, sich mit der Realität des Sterbens auseinander zu setzen, sie machen sich mehr Gedanken und wollen eher bewusst und begleitet sterben; Männer neigen stärker zur Verdrängung.

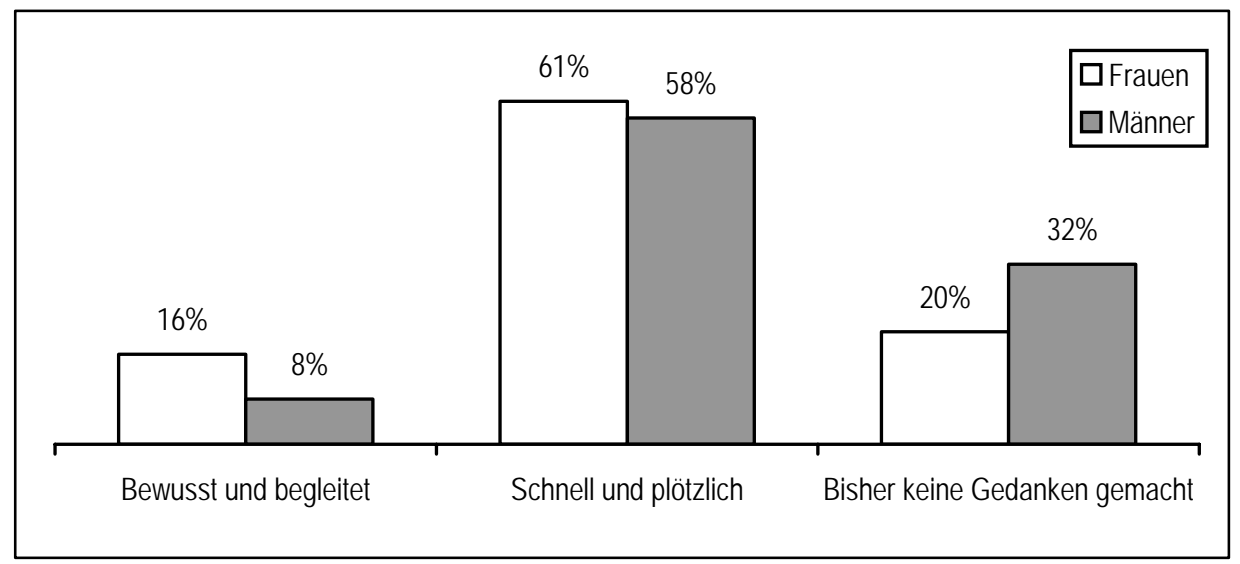

Abb. 4: Eigener Sterbewunsch in Abhängigkeit vom Geschlecht (Quelle: Deutsche Hospizstiftung, Emnid-Umfrage 2001)

Auch die Sterbesituation in Deutschland (vgl. Abb. 5) wird eher idealisiert, 1996 und 2001 glaubte jeder Zweite, dass die meisten Menschen in Deutschland human, natürlich und würdig sterben. Das Sterben der anderen wird idealisiert, immerhin sind $40 \%$ schon eher kritisch. 


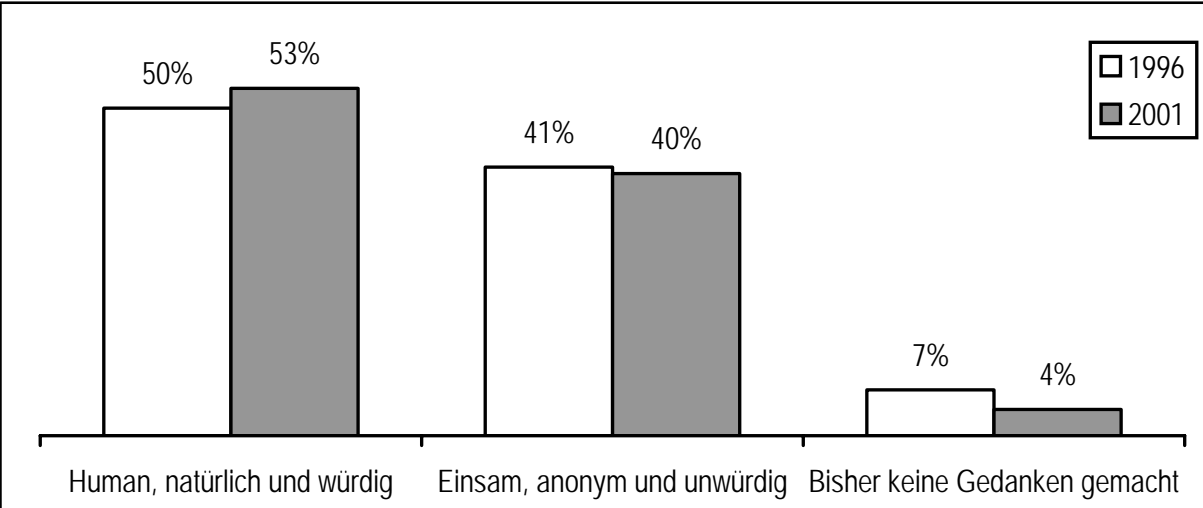

Abb. 5: Wie sterben die meisten Menschen in Deutschland? (Quelle: Deutsche Hospizstiftung, Emnid-Umfrage 2001)

Wie erwartet verleugnen die Männer das Sterben in Deutschland stärker als die Frauen, zwei Drittel von ihnen beurteilen das Sterben als „human“ oder geben an, sich keine Gedanken gemacht zu haben, bei den Frauen ist das eher die Hälfte der Befragten (vgl. Abb. 6). Aber wie immer bei ambivalenten Themen, ergibt sich auch eine neue Tendenz, man beginnt, sich mit anderen Aspekten des Sterbens auseinanderzusetzen.

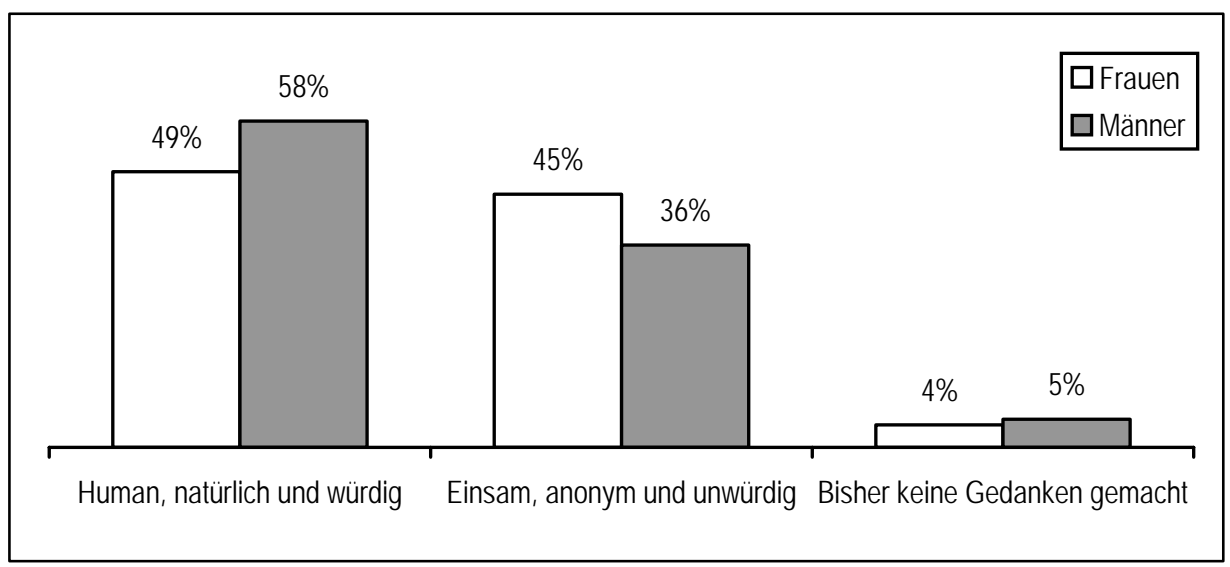

Abb. 6: Meinungen zum Sterben in Deutschland in Abhängigkeit vom Geschlecht (Quelle: Deutsche Hospizstiftung, Emnid-Umfrage 2001)

So ist der Bekanntheitsgrad des Begriffes „Hospiz“ in der Zeit von 1996 bis 2001 immer größer geworden (vgl. Abb. 7), er hat sich enorm gesteigert, wenn auch nur 
jeder Dritte weiß, was ein Hospiz ist. Das setzt sich fort in Bezug auf die geschlechtsspezifischen Unterschiede. Mehr als ein Drittel der Frauen kennt den Begriff „Hospiz“, jedoch nur jeder 4. Mann (vgl. Abb. 8).

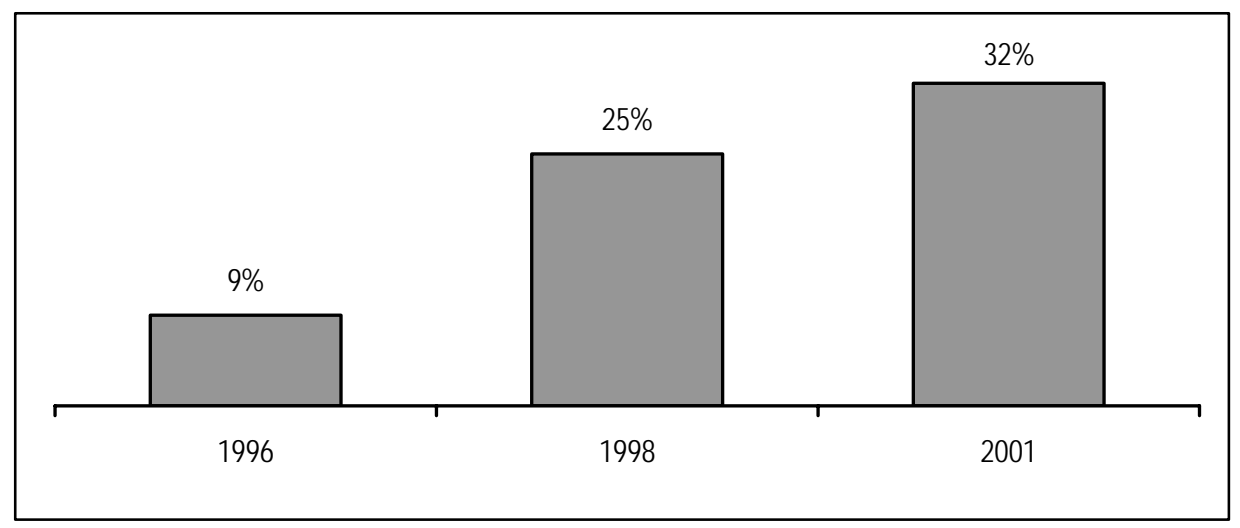

Abb. 7: Bekanntheitsgrad des Begriffs „Hospiz“ (Quelle: Deutsche Hospiz-Stiftung, Emnid-Umfrage 2001)

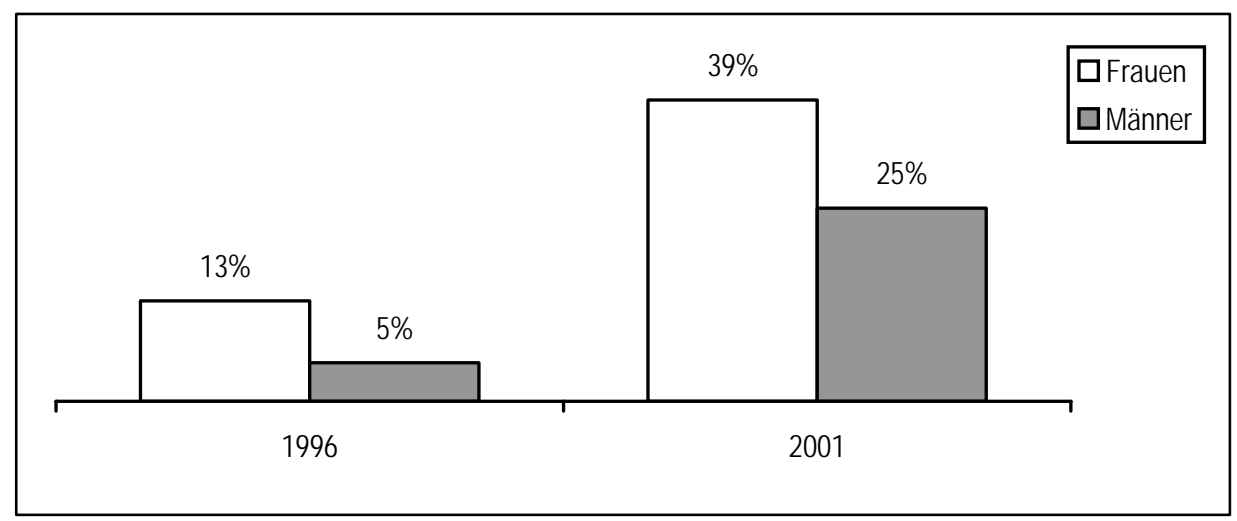

Abb. 8: Bekanntheitsgrad des Begriffs „Hospiz“ in Abhängigkeit vom Geschlecht (Quelle: Deutsche Hospiz-Stiftung, Emnid-Umfrage 2001)

Grenzsituationen par excellence, Erschütterung aller Gewissheiten, Stabilitätsverlust und Angst: Das hat gemeinhin vor Augen, wer an das Sterben denkt. In einer Gesellschaft wie der deutschen, die keine sozial geteilten Sinngebungen mehr kennt, sieht sich das Individuum mit dem Tod alleingelassen und überfordert. Dem einzelnen Menschen steht kaum mehr eine Möglichkeit zur Verfügung, das Lebensende in einen Sinnzusammenhang zu integrieren, der gesellschaftlich abge- 
sichert über den Alltag hinausweist. Ein Vakuum ist an die Stelle der Religion getreten, und die Kirche hat ihre allumfassende Funktion als Sinnstifterin verloren.

Die Deutung des Todes ist zur innersubjektiven oder zumindest privaten Angelegenheit geworden. In seinem Essay über den Tod schreibt der Soziologe und Sozialphilosoph Norbert Elias, es verbreite sich „die Vorstellung, dass ein Mensch - man selbst - ganz für sich allein einen Sinn haben müsse."1 Die Fragilisierung und Gefährdung, die der moderne Tod auslöst, verstärken sich infolge des demografischen Umstandes, dass das Sterben heute eher zum „seltenen Sterben“ geworden ist. Vor über 100 Jahren hat ein Mensch mit 35 Jahren in der Regel mehrere schwere Epidemien überlebt und eine Reihe von Geschwistern, Freunden oder Altersgenossen sterben sehen. Seither jedoch ist der Tod zu einem seltenen Ereignis geworden, denn Menschen sterben immer später. Gegenwärtig beläuft sich die Lebenserwartung in den modernen Industriegesellschaften in Europa auf 75 bis 85 Jahre. Man hat geschätzt, dass in einer Familie in Deutschland heute ungefähr alle 10 bis 15 Jahre ein Todesfall vorkommt, der das Sterben als ein Phänomen in seiner ganzen Tragweite bewusst werden lässt. Menschen, die so selten mit dem Tod von Mitmenschen konfrontiert sind, haben vergleichsweise geringe Chancen, sich Fähigkeiten anzueignen, welche die Verarbeitung dieser Erfahrung erleichtern. Zu einem Inhalt der Sozialisation können Sterben und Tod unter den Bedingungen des seltenen Sterbens nicht werden. Hinter allen Diskussionen, die in den letzten Jahren geführt worden sind, steht das Erleben des Unvermögens, angesichts des Todes Gelassenheit zu wahren. Dieses Unvermögen ist zu einem zentralen Merkmal modern-städtischer Lebenszusammenhänge geworden. In der Beschäftigung mit Sterbewirklichkeiten in der heutigen Zeit muss immer auch der historische Hintergrund mitbedacht werden. So möchte ich ein paar kurze Aspekte beleuchten, wie die Tabuisierung des Sterbens in der Neuzeit sich etabliert hat, um in der zweiten Hälfte des 20. Jahrhunderts durch eine neue Form der Institutionalisierung des Sterbens abgelöst zu werden.

Den Hintergrund bildet die historische Entwicklung der ständigen Erhöhung der Lebenserwartung und als Folge davon der Herausbildung der Form des langen Sterbens $^{2}$. Betrug die Lebenserwartung noch Ende des 19. Jahrhunderts 42 Jahre, war sie schon 1980 bei 76 Jahren und hat um 2000 ungefähr 80 Jahre erreicht. Dahinter steht eine Entwicklung, die man auch bezeichnen kann als eine „Pazifizierung des Todes“, wie es Elias genannt hat. Große Schicksalsschläge, unvorhersehbare Einbrüche, physische Bedrohungen, Hungerkatastrophen und Epidemien gehen zurück und haben eine relative Befriedung des Sterbens zur Folge. In de-

1 Elias N (1982): Über die Einsamkeit der Sterbenden in unseren Tagen. Suhrkamp, Frankfurt, S. 85

2 Streckeisen U (2001): Die Medizin und der Tod. Über berufliche Strategien zwischen Klinik und Pathologie. Opladen, Leske und Budrich; Göckenjan G \& Dreßke S (2002): Wandlungen des Sterbens im Krankenhaus und die Konflikte zwischen Krankenrolle und Sterberolle. Österreichische Zeitschrift für Soziologie, 27, 80-96 
mografischer Hinsicht ${ }^{3}$ spielen neben der Erhöhung der Lebenserwartung vor allem die Veränderung der Todesursachen und der Sterblichkeit eine große Rolle. Diese Entwicklung verläuft in drei Abschnitten:

1. Es gibt eine hohe Sterblichkeit in der Vor- und Frühindustrialisierung, die mit hoher Kinder- und Säuglingssterblichkeit zusammenhängt. Ansonsten sterben die Menschen an Epidemien und an Hungersnöten.

2. Im 19. Jahrhundert nimmt die Sterblichkeit langsam ab und die Lebenserwartung steigert sich. Das Bevölkerungswachstum wird kontinuierlicher und beschleunigt sich. Die Epidemien verschwinden, und die Infektionskrankheiten verwandeln sich in handhabbare, behandelbare Krankheiten, die Qualität der Ernährung verbessert sich, ebenfalls wird Hygiene und Gesundheitsförderung verbessert.

3. Im Zuge des epidemiologischen Übergangs werden jetzt Infektionskrankheiten durch degenerative und chronische Krankheiten ersetzt. Während um 1900 Lungenentzündung, Influenza, Tuberkulose, Magenschleimhautentzündung, Zwölffingerdarmentzündung, ansteckende Kinderkrankheiten und anderes als Todesursache noch weit verbreitet sind, stehen jetzt in der zweiten Hälfte des 20. Jahrhunderts Tumorkrankheiten, Herzkrankheiten und gefäßbedingte Schädigungen des zentralen Nervensystems (z.B. Schlaganfall) in den ersten Rängen der Todesursachen. Im Gegensatz zu Infektionskrankheiten kommen degenerative und chronische Krankheiten eher später im Leben vor. Ein gewisses Alter muss erreicht sein, damit sie überhaupt auftreten und jemand daran sterben kann. Als typische, direkt altersgebundene Krankheiten gelten z.B. die Demenz und die AlzheimerKrankheit, die von Gedächtnisschwund bis hin zu totaler Unselbständigkeit und Zerstörung der Persönlichkeit reichen können. So werden Alterskrankheiten jetzt unterschieden in:

- Alternde Krankheiten: Das sind Organfunktionsstörungen im Alter, die durch krankhafte Prozesse verursacht sind, die das Altern schon von frühen Lebensphasen her begleiten und einen chronischen Verlauf aufweisen.

- Primäre Alterskrankheiten: Das sind Krankheiten, die im Alter erstmals auftreten und in ihrer Häufigkeit an das höhere Lebensalter gebunden sind (z.B. Altersdiabetes, Parkinson).

- Krankheiten im Alter: Das sind Krankheiten, die in allen Lebensabschnitten auftreten, aber unter den Bedingungen der biologischen Situation des alternden Menschen atypisch verlaufen, höhere Risiken mit sich bringen etc., wie z.B. Infektionen, beispielsweise Lungenentzündung, Influenza.

3 Imboff A (1981): Die gewonnenen Jahre. Von der Zunahme unserer Lebensspanne seit dreihundert Jahren oder von der Notwendigkeit einer neuen Einstellung zu Leben und Sterben. C.H. Beck, München 
Eine Kombination aus allen drei Krankheiten können wir in den sieben apokalyptischen Reitern für das Alter sehen: Arteriosklerose, Bluthochdruck, Altersdiabetes, Fettleibigkeit, Formen seniler Demenz, Krebs und eine geschwächte Immunabwehr. Oft leiden alte Menschen an mehreren dieser Erkrankungen, nicht selten kommt es vor, dass Schwerstkranke an allen sieben gleichzeitig leiden ${ }^{4}$.

In der Zeit des epidemiologischen Übergangs nimmt die Sterblichkeit ab, pendelt sich auf einem niedrigeren Niveau ein, und die durchschnittliche Lebenserwartung steigt an. Die Lebenserwartung hat sich in Deutschland in sämtlichen sozialen Schichten erhöht, was die gegenwärtige Sterbewirklichkeit bestimmt, nämlich für sämtliche soziale Schichten gilt mehr oder weniger, dass ein „langes Leben“ immer mehr zum Allgemeingut geworden ist ${ }^{5}$. Dies hängt mit der Ausdehnung des wirtschaftlichen Wohlstands, auch mit der Ausweitung der medizinischen Grundversorgung auf die gesamte Bevölkerung zusammen. Vorbeugemaßnahmen, wie aber auch die Heilung von Krankheiten, die früher tödlich verlaufen wären, sind jetzt allen sozialen Bevölkerungsgruppen zugänglich. Die Folge ist, was für die gegenwärtige Situation in Deutschland gilt: Der „vorzeitige“ Tod von früher ist radikal zurückgedrängt worden und ein immer größerer Teil der Bevölkerung kann mit einem hohen Alter rechnen.

Aber die Verbreitung und medizinische Kontrolle und Steuerung von degenerativen und chronischen Krankheiten erhöht nicht allein die Lebenserwartung, sie verändert auch den Sterbeprozess. Mit dem Aufkommen der chronischen Krankheiten ist ein neues Phänomen entstanden: das „lange Sterben“ - oder anders ausgedrückt: die „lange Sterbedauer“ bzw. „das langsame Sterben“ sind zum Bestandteil des Lebenslaufs geworden ${ }^{6}$. Chronische und degenerative Krankheiten konfrontieren die Betroffenen mit der Möglichkeit eines langen Leidens und eines damit möglicherweise zusammenhängenden lang andauernden Sterbeprozesses. Das heutige Sterben, das wir in Deutschland vor allem in den Sterbeorten des Krankenhauses und der Alten- und Pflegeheime, aber auch zu geringeren Teilen in der häuslichen Umgebung, in den Familien beobachten können, kann manchmal Wochen, Monate, oft Jahre andauern, im Gegensatz zu früher, wo etwa die Inkubationszeit der Pest ein bis fünf Tag in Anspruch nahm und der Tod bereits innerhalb von einer halben Woche eintrat, bei Cholera zwischen Ansteckung und Ausbruch zwei bis fünf Tage lagen und in zwei bis fünf Tagen mit dem Ableben zu rechnen war.

Zur Sterbewirklichkeit gehört heute auch eine Veränderung des Sterbegriffs ${ }^{7}$. Man muss verschiedene Formen des Sterbens auseinander halten, die vielleicht als einziges gemeinsames Merkmal die kalendarisch-zeitliche Ausdehnung kennen. So unterscheidet man heute den sog. „engen Sterbegriff“, womit das Im-Sterben-

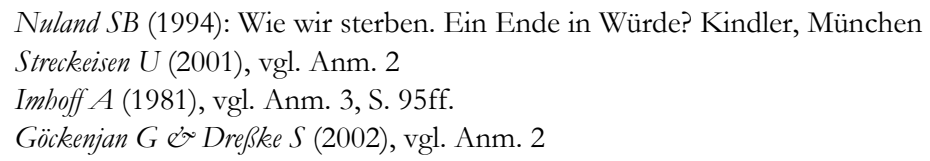


Liegen gemeint ist, zu welchem in der Regel die Agonie gehört. Mit dem „weiten Sterbebegriff“ ist der Prozess des Übergangs vom Leben zum Nicht-mehr-Leben angesprochen, es geht um die Schlussphase im Lebens- und Alterungsprozess, die schwierig einzugrenzen und in der medizinischen Praxis - insbesondere im Krankenhaus - oft umstritten ist, weil sie kurativ im Sinne der Lebenserhaltung definiert wird. Schließlich kann man das Sterben in einem „sehr weiten“ Sinne verstehen, nämlich jene Zeit, während derer das Lebensende zur zentralen Einstellung eines Menschen wird. Das Sterben bildet im letzteren Fall eine lebenszyklische Phase mit dem wichtigen Merkmal, dass sich der Sterbende auf den Tod ausrichtet; dieser Sterbebegriff trifft eher auf moderne Formen der Palliativ- und Hospizbewegung zu.

Ähnliche Unterscheidungen werden vorgenommen in Bezug auf die klinische Praxis, wenn unterschieden wird ${ }^{8}$ :

- die erwartete schnelle Sterbebahn, wie z.B. der Unfalltod oder

- die unerwartete schnelle Sterbebahn, wie z.B. Komplikationen nach einer Routineoperation und

- die zögernde Sterbebahn im Falle von chronischen Erkrankungen.

Ähnlich kann unterschieden werden zwischen dem plötzlichen Tod (z.B. Lungenembolie), der akuten Erkrankung mit anschließendem Tod (z.B. Herzinfarkt), dem akuten tödlichen Schub bei chronischer Krankheit und dem langsamen Sterben an chronischer Krankheit wie etwa bei bestimmten Tumorerkrankungen und der Kombination von mehreren chronischen Krankheiten.

In der Gegenwart ist die Hauptform das „lange Sterben“, das für die Sterbewirklichkeit in Deutschland gilt. Aus der Häufigkeitsverteilung der Todesursachen ist dies zu erkennen: Infektionserkrankungen machen heute nur noch einen sehr geringen Anteil an den Todesursachen aus - nur im höheren Alter spielen sie eine signifikante Rolle. Junge Menschen sterben heute nicht an Infektionen, sondern eher durch Unfälle und Selbstmord. Aber die Häufigkeit dieser Todesursache ist weit geringer als die damalige Häufigkeit des Todes in Folge von Infektionen. Degenerative und chronische Krankheiten, die in den oberen Rängen stehen, sind mit einem Tod im hohen Alter verbunden. Aber auch bei degenerativen und chronischen Krankheiten kann man in den letzten Jahrzehnten einen Rückgang des Sterberisikos beobachten: Herzinfarkte und Tumore führen heute nicht mehr so schnell zum Tod, vor allem bei Frauen ist die Sterblichkeit infolge von Tumorerkrankungen zurückgegangen, während die Todesfälle bei Männern infolge von Lungenkarzinomen angestiegen sind. In den Industriegesellschaften - und damit auch in Deutschland - hat sich die Wahrscheinlichkeit, nicht nur alt zu werden, sondern sehr alt zu werden, in den vergangenen Jahrzehnten stark erhöht. Zum ersten Mal in der Geschichte unserer Gesellschaft wird Sterben zur Angelegenheiten von in der Mehrheit alten und hoch betagten Menschen. Das demografische

8 Corbin JM (1993): Chronizität und das Verlaufskurvenmodell. Syst Fam (System Familie), 6(3), 151-160 
Altern, die Vergrößerung des Anteils alter Menschen hat zwar bereits in der Zeit nach dem 2. Weltkrieg eingesetzt, war aber zunächst vor allem eine Folge der Reduktion der Anzahl der Kinder und Jugendlichen und weniger eine Folge des Anwachsens der Alten. Erst in den letzten Jahrzehnten ergibt sich auch von der Spitze her eine gewisse Alterung: Während sich früher eine Generation von gleichzeitig Geborenen im Verlaufe des Lebens kontinuierlich verkleinerte, (z.B. bei jedem Klassentreffen fehlten einige mehr), erreicht die Generation jetzt als Ganze ein relativ hohes Alter und verliert dann gesamthaft innerhalb kurzer Zeit das Leben.

Dies hat auch Folgen für die Sterbewirklichkeit: Die Erhöhung der Lebenserwartung und das damit verbundene lange Sterben hat zu verschiedenen Aspekten des sozialen Wandels beigetragen, unter anderem zur emotionalen Betroffenheit und Bedeutung des Todes eines nächsten Angehörigen. In der Familie hat die Verwandtschaft infolge der gestiegenen Lebenserwartung an Bedeutung verloren, dafür hat die Kernfamilie an Eigenständigkeit gewonnen, da sie als soziale Einheit durch Todesfälle weniger gefährdet ist. Angesichts eines langen Lebens ist es möglich, sich bei der Partnerwahl Zeit zu lassen und Gesichtspunkte der Zuneigung zu berücksichtigen. Ferner können sich zwischen Eltern und Kindern enge Gefühlsbeziehungen entwickeln. Die herausbildende Intensität des familialen Gefühlslebens betrifft jetzt sämtliche emotionale Dimensionen, sie führt von Liebe und Glück über den Hass bis hin zur Trauer. Bezogen auf Sterben und Tod hat dieses nun auch für die Sterbewirklichkeit zur Folge, dass Angehörige eines sterbenden oder gestorbenen Menschen emotional immer mehr berührt sind. Je weniger der Verstorbene ersetzt werden kann und je wichtiger seine emotionale Funktion im Alltag gewesen ist, umso schmerzhafter ist der Loslösungsprozess. Obendrein ist die Kernfamilie gegenüber den verlängerten Prozessen von Sterben und Tod in Bezug auf ihre subjektiven und objektiven Toleranzschwellen stärker betroffen: Je länger eine Krankheit dauert und je schwerwiegender diese ist, je mehr Pflegeaufwand diese erfordert, umso weniger hat die Familie subjektive und objektive Ressourcen zur Bewältigung dieses Prozesses, insbesondere wenn dieser Prozess den Wendepunkt zum Sterben erreicht hat ${ }^{9}$. Dies trägt vor allem auch dazu bei, dass sich der Ort des Sterbens von der Familie immer stärker in andere Institutionen der Gesellschaft verlagert und immer weiter verlagern wird, insbesondere in die Bereiche der Alten- und Pflegeheime und des Krankenhauses sowie der Palliativ- und Hospizeinrichtungen.

Die Erhöhung der Lebenserwartung und der damit einhergehende Anstieg des Anteils alter Menschen an der Bevölkerung ist Bestandteil eines einschneidenden Prozesses gesellschaftlicher Umwälzung, der auch die Vorstellung vom Alter und

9 Rohde JJ (1975): Strukturelle Momente der Inhumanität einer humanen Institution. In: Schwitajewski H, Rohde JJ (Hrsg.): Berufsprobleme der Krankenpflege. Urban \& Schwarzenberg, München, S. $12-14$ 
der letzten Lebensphase im hohen Maße verändert hat ${ }^{10}$. Unterschied man den Lebenslauf in Form einer Dreiteilung in:

1. Kindheit/Jugend (Vorbereitungsphase)

2. aktives Erwachsenenleben (Aktivitätsphase) und

3. Alter (Ruhephase),

so muss gerade die letztere in sich neu differenziert werden. Galt lange als Vorstellung vom Alter eine Defizitnorm, in dem Sinne, dass man immer mehr Funktionen und Fähigkeiten im Alter verliert, so ist heute das Ressourcenmodell im Sinne der Erhaltung und Entwicklung von spezifischen Leitlinien und Bewältigungsmöglichkeiten - im Vordergrund. Aber das Alter hat sich in seiner Ausdifferenzierung zwischen aktivem Ruhestandsalter und dem neuen vierten Alter, dem Greisen- bzw. Hochbetagten-Alter, verändert in dem Sinne, dass man heute das dritte Alter bezeichnet als „,autonome Phase“, insofern als nach der Pensionierung oder Berentung diese alten Menschen ein unabhängiges eigenes aktives Leben führen und gestalten können, was auch zur Veränderung der Freizeitaktivitäten geführt hat. An dieses entfaltungs- und progressionsversprechende dritte Alter, das mittlerweile zum Lebenslauf einer großen Mehrheit der Bevölkerung gehört, reiht sich nun das vierte Alter an, in welches immer mehr Menschen eintreten. Dieses vierte Alter ist jetzt durch Abhängigkeit gekennzeichnet: Der Mensch vermag seinen Alltag nicht mehr alleine zu bewältigen und ist auf die Hilfe von anderen angewiesen. Oft ist der Übergang in dieses vierte Alter mit der regelmäßigen Inanspruchnahme von professioneller Hilfe verbunden und/oder bringt einen Wechsel vom privaten Wohnen hin zu einer organisierten Wohnform (Pflegeheime und anderes) mit sich: Nicht zufällig steigt der Anteil der über 75-Jährigen exponentiell an in Bezug auf den Übertritt in Alten- und Pflegeheime.

Mit der Verlängerung der Lebenserwartung, dem gestiegenen Alter, der Zunahme chronischer und degenerativer Erkrankungen und damit auch der Verlängerung und Intensivierung der Sterbeprozesse ist es zunehmend auch zu einer gestiegenen Medikalisierung des Sterbens gekommen, in dem Sinne, dass der Prozess chronischer Erkrankung zunehmend Gegenstand der Medizin mit ihren gestiegenen Diagnostik- und Behandlungsmöglichkeiten - insbesondere auch den lebensverlängernden Techniken - geworden ist. Das bedeutet, dass zunehmend Prozesse des Alterns medizinalisiert und entsprechend Gegenstand von Experten geworden sind, was wiederum zu einer zunehmenden Distanzierung gegenüber Sterben und Tod geführt hat. In dem Maße, wie chronische und degenerative Erkrankungen zu einem zentralen Objekt der Medizin geworden sind, sind die Endphasen dieser Krankheitsverläufe aus dem gesellschaftlichen Leben ausgegrenzt und spezialisierten Einrichtungen wie dem Krankenhaus überantwortet worden. Das hat zur Folge, dass Sterben und Tod zunehmend an professionelle Institutionen wie Medizin, Krankenhaus, Pathologie, Leichenbestatter delegiert

10 Streckeisen $U$ (2001), vgl. Anm. 2 
wurden. So werden Sterbende hygienisch in Krankenhäuser gebracht und Verstorbene geruchlos ins Grab expediert. Die Isolierung der Sterbeprozesse, die Individualisierung, Vereinsamung und Vereinzelung des sterbenden Kranken sind $\mathrm{zu}$ vorherrschenden Phänomen geworden und bilden wohl auch den Hintergrund der Angst vieler Menschen vor der Macht der technologischen Medizin.

Zwar ist der Wunsch, zu Hause zu sterben, weit verbreitet (s. dazu Abb. 9), doch immer öfter verbringen Menschen die letzten Phasen ihres Lebens oder längere Episoden in den letzten Lebensabschnitten außerhalb ihres Privatbereichs, und Sterben und Todeseintritt erfolgt meist auch in außerfamilialen Institutionen. Unter den Organisationen, in denen Sterben stattfindet und der Tod eintritt, dominiert das Krankenhaus.

- Zurzeit sterben pro Jahr ca. 850.000 Menschen in Deutschland.

- 80 bis 90 Prozent der Befragten wünschen sich ein Sterben zu Hause.

- Doch die meisten - zwischen 50 und 58 \% - sterben im Krankenhaus.

- 18 bis 25 Prozent sterben in Einrichtungen der Altenhilfe.

Abb. 9: Sterben in Deutschland

Es ist schwierig, die exakte Verbreitung des Sterbens im Krankenhaus, Altersheimen, Hospizen etc. und im Privatbereich empirisch zu erfassen, denn dazu bräuchte man Daten zur Verteilung der Sterbenden auf diese sozialen Orte. Solche exakten Informationen liegen indessen nicht vor; in Deutschland gibt es eine sträfliche Vernachlässigung der Epidemiologie, die darüber Aufschluss geben könnte. Außerdem ist es oft schwierig, solche Daten zu erheben, da Schwerkranke während der Zeit des Sterbeprozesses oftmals ihren Aufenthaltsort wechseln, z.B. vom privaten Zuhause oder Heim ins Krankenhaus gehen, dann wieder zurückkehren und sich kurz vor dem Sterben ggf. noch einmal ins Krankenhaus begeben. Als Haupttendenz können wir dennoch feststellen, dass es eine deutliche Erhöhung des Anteils der im Krankenhaus gestorbenen Personen gibt. Für Deutschland können wir folgende Entwicklung sehen ${ }^{11}$ : In drei Jahrzehnten von den 50er Jahren bis in die 90er gibt es einen steigenden Anteil der im Krankhaus erfolgten Sterbefälle für die Bundesrepublik Deutschland:

1954 sind es $28 \%$,

1958 sind es $42 \%$,

1968 sind es $44 \%$,

1978 sind es $59 \%$,

1988 sind es $65 \%$.

Gegenwärtig sterben im Krankenhaus zwischen 50 und $58 \%$, das ist von demografischen Schwankungen abhängig. In Einrichtungen der Altenhilfe sterben zwischen 18 und $25 \%$.

11 Rohde JJ (1975), vgl. Anm. 9; Göckenjan G \& Dreßke S (2002), Streckeisen U (2001), vgl. Anm. 2 
Die Veränderungen sind vor allem bedeutsam in Bezug auf den privaten Bereich, weil der Prozentsatz der im Altenheim oder im Krankenhaus Sterbenden in den letzten Jahrzehnten zugenommen hat, und zwar komplementär zu einer Abnahme der Sterbefälle, die zu Hause eintraten. Dieses hat vor allem zu tun mit der systematischen Planung und dem Ausbau des Alters- und Pflegeheimbereichs, der in den später 70er Jahren einsetzte, immer mehr Bedeutung erlangt hat und sich zunehmend heute auch in seinem Sektor zwischen öffentlichen und privaten Einrichtungen differenziert hat. Das heißt, es kann damit gerechnet werden, dass viele Sterbeprozesse in solche spezialisierten Einrichtungen verlegt werden, auch aus dem Krankenhaus heraus verlegt werden, um schließlich in der kurzen Phase des akuten Sterbens und Todes dann wieder ins Krankenhaus zurücküberwiesen zu werden.

Die Orte der Sterbewirklichkeit sehen für Frauen und Männer teilweise ähnlich, teilweise aber auch verschieden aus. Die Abnahme des Sterbens zu Hause verläuft für die beiden Geschlechter bis zu einem bestimmten Alter fast gleich, für die Männer allerdings auf einem bis zu $10 \%$ höheren Niveau:

Bei Frauen geht der Anteil der Zu-Hause-Sterbenden zunehmend in den letzten Jahrzehnten zurück, wesentlich stärker als bei Männern. Viele Frauen werden bekanntlich älter als Männer und übernehmen - in Fortführung ihrer familialen Arbeitsrolle - häufig die materielle und immaterielle Sorge für den alternden, kranken und dann sterbenden Partner. Während für Männer nach dem Übertritt aus dem Erwerbsleben ins Rentendasein ein Defizit an Aufgaben gegeben sein kann, trifft für viele Frauen in diesem Alter eher das Gegenteil zu: Nicht nur fehlt oft der markante Einschnitt der Statuspassage Berentung oder Pensionierung, sondern ein abhängig werdender Ehemann lässt für die Frau innerfamilial ganz neue Aufgaben entstehen. Man kann in diesem Zusammenhang davon sprechen, dass die innerfamiliale Rollenkonfiguration der Ehefrau sich im Alter erweitert, während sich jene des Mannes reduziert.

Im Gegensatz zum Sterben und Tod zu Hause, sterben Frauen häufiger im Altersheim als Männer. Das lässt sich mit der höheren weiblichen Lebenserwartung erklären. Das heißt, je älter jemand ist, desto geringer ist die Chance, zu Hause zu sterben. Infolge der höheren Lebenserwartung von Frauen ist der Tod im Altersheim mehr ein kulturell weibliches als männliches Phänomen.

In Bezug auf das Sterben im Krankhaus sind kaum geschlechtsspezifische Unterschiede auszumachen, beide Anteile oszillieren zwischen 50 und 58 Prozent.

In Bezug auf die Stadt/Land-Unterschiede ist erwartungsgemäß festzustellen, dass in den städtischen Zentren das Krankenhaus der hauptsächliche gesellschaftliche Ort des Sterbens ist, während auf dem Lande der Sterbeort zu Hause noch etwas mehr ins Gewicht fällt. Allerdings ist auch hier zu beobachten, dass eine Angleichung stattfindet; auch im ländlichen Bereich reduziert sich immer mehr das Sterben im häuslichen Bereich. Es erfolgt eine Verlagerung hin zum Kranken- 
haus. Das Krankenhaus ist somit also der zentrale gesellschaftliche Ort des Sterbens und des Todes geworden.

Ob jemand im Krankenhaus stirbt oder nicht, hängt allerdings deutlich mit der Todesursache zusammen. Vor allem Krebserkrankungen führen zum Tod im Krankenhaus. Für Herz- und Kreislaufkrankheiten und den gewaltsamen Tod gilt das Gegenteil. Dass Unfälle, aber auch Suizid und Tötung keinen Todeseintritt im Krankenhaus mit sich bringen, mag damit zusammenhängen, dass für die Einlieferung ins Krankenhaus oftmals keine Zeit mehr bleibt, da die Zeitspanne zwischen dem Einsetzen der lebensgefährdenden Bedingungen und dem Eintritt des Todes kurz ist. Dies dürfte auch für gewisse Herz- und Kreislauferkrankungen gelten, wie z.B. Infarkt, Angina pectoris. Bei tumorkranken Menschen ist die Krankenhauseinweisung und das Sterben im Krankenhaus eher der Fall. Auch wenn Tumorkranke zwischen Krankenhaus und dem privaten Zuhause pendeln, was während der langen Krankheits- und Sterbephase oft der Fall ist, befinden sie sich in der Terminalphase doch oft dann im Krankenhaus, da hier eine intensive medizinische Betreuung möglich ist.

In Bezug auf das Alter fällt auf, dass der Anteil der im Krankenhaus Sterbenden ab 50 Jahren zunächst ansteigt, ab 60 Jahren eher flach verläuft und ab 80 Jahren deutlich zurückgeht. Das heißt der Satz: „Je älter, desto weniger wird im Krankenhaus gestorben“, gilt nur für die Gruppe der Hochbetagten. Das mag sich dadurch erklären, dass Hochbetagte eher als andere Betagte im Altersheim wohnen und bis zum Tode dort bleiben. Hier zeigt sich übrigens auch, dass bei einer guten personellen Ausstattung und einer ausreichenden Ausrüstung an Ressourcen Alten- und Pflegeheime ein geeigneter Ort sein können für Sterbebegleitung, allerdings nicht, wenn Personal und Ressourcen verknappt werden.

Das Alten- und Pflegeheim ist also an zweiter Stelle als gesellschaftlicher Sterbeort zu nennen. Je älter jemand ist, desto eher stirbt er oder sie im Heim. Die Todesursache hat keinen Einfluss. Nur wird deutlich, dass Krebskranke nicht im Heim sterben, sondern zumeist in einer spezialisierten Einrichtung des Krankenhauses. Auch der Familienstand ist hierbei von Belang, Alleinstehende bzw. Nicht-mehr-Verheiratete sterben häufiger als andere im Altersheim. Es handelt sich hier um Personen, die keine privaten Betreuungsmöglichkeiten haben und allein sind. Nicht von ungefähr sind die im Heim Sterbenden auch eher weiblichen denn männlichen Geschlechts. Auch soziale Faktoren spielen in Bezug auf den Sterbeprozess im Heim eine Rolle, sozial schlechter gestellte Menschen sterben eher häufig im Alten- und Pflegeheim, sozial besser Gestellte eher selten, es sei denn, sie befinden sich in spezialisierten Altersresidenzen mit angeschlossenen Pflege- und Behandlungsmöglichkeiten. Besser situierte Menschen haben allerdings auch einen besseren Zugang zu medizinischen Möglichkeiten und Versorgung als sozial schlechter gestellte. 
Der im Altersheim sterbende Mensch ist also häufig betagt bis hochbetagt, nicht mehr verheiratet bzw. alleinstehend, und oft gehört er eher den unteren sozialen Schichten an.

Ich möchte mich jetzt dem Krankenhaus als dem zentralen gesellschaftlichen Sterbeort zuwenden. Die Situation um Sterben und Tod ist im Krankenhaus ein Feld prekären sozialen Handelns. Weder die Institution Krankenhaus als Ganzes, noch ihre Rollenträger sind auf den Umgang mit Sterbenden eingestellt, das gilt nach wie vor - trotz aller Diskussionen über Palliativmedizin und Hospizbewegung. Sterben im Krankenhaus ist strukturell und organisatorisch nicht vorgesehen. Sterbende sind zuerst einmal Kranke und werden auch als solche definiert. Sie werden den kurativen Zielsetzungen unterworfen im Sinne der Krankheitsbehandlung. Das zeigt sich schon daran, dass eine medizinische Definition des Sterbens im Krankenhaus nicht existiert. Wie wird Sterben definiert und wie wird es zeitlich eingegrenzt?

Nehmen wir die Definition der Bundesärztekammer: „Ein Sterbender ist ein Kranker oder Verletzter mit irreversiblem Versagen einer oder mehrerer vitaler Funktionen, bei dem der Eintritt des Todes in kurzer Zeit zu erwarten ist.“ Diese Definition beschreibt einen begrenzten zeitlichen Ausschnitt (,in kurzer Zeit") und bleibt dabei dennoch auffällig vage. Zugleich ist der Sterbeprozess mit dem Ausfall vitaler Funktionen allein physiologisch-funktionell bestimmt. Wichtig ist das Kriterium der Irreversibilität, wenngleich es nicht näher ausgeführt ist. Zugespitzt formuliert könnte man sagen, - und so wird es von einigen medizinischen Autoren auch gesagt - dass der Sterbeprozess genau genommen immer nur medizinisch gesehen ex post zu bestimmen sei: „Das Sterben wird von seinem Ende her als Sterben begreifbar, niemals zuvor. Wir wissen erst dann, ob jemand das Sterben erfuhr, wenn er schlussendlich gestorben ist. Woher sollen wir aber nun wissen, wann dieses Sterben begonnen hat, welche Erfahrungen also dem reversiblen Zustand schwerster Krankheit und welche Erfahrung dem irreversiblen Prozess des Sterbens selbst zugerechnet werden dürfen?"“12 Für eine theoretische Diskussion mag eine solche Definition plausibel sein, aber für die in der realen Situation handelnden Personen auf einer Krankenstation in einem Krankenhaus ist dies jedoch nicht praktikabel. Die Sterbesituation stellt sich vielmehr als ein unsicheres Feld medizinischen und sozialen Handelns dar, und die Akteure müssen ihr Handeln an einer bestimmten Einschätzung des Prozesses orientieren. In etlichen Fällen wissen die Handelnden, dass ein Patient sterben wird, in anderen Fällen wird das Wissen eher ein Ahnen sein, in wieder anderen werden sie durch das Sterben überrascht. Bei allen Untersuchungen, die leider relativ spärlich vorhanden sind, wird immer wieder gefunden, dass für die meisten Handelnden im Krankenhaus die wahrscheinlich entscheidendste Phase des Sterbens dann beginnt, wenn die verschiedenen Gruppen im Krankenhaus - insbesondere Ärzte

12 Macho TH (1987): Todesmetaphern - Zur Logik der Grenzerfahrung. Ed. Suhrkamp 1419, Neue Folge Band 419. Suhrkamp, Frankfurt/M., S. 28 
und Pflegepersonal - das Gefühl entwickeln, bei aller Maximaltherapie für den Patienten „,nichts mehr tun“ zu können. Das bedeutet, dass für die Handelnden der Patient sich weder auf natürliche Weise, noch unter Einsatz aller verfügbaren ärztlichen Mittel noch einmal erholen wird. Aber wann wird der Wendepunkt von einem therapeutischen Handeln und der Beeinflussung durch kuratives Therapieren bei einer Krankheit bei einem Schwerstkranken zu erkennen sein? Wann verändert sich die Krankheit in einen Prozess des Sterbens? Denn tritt ein solcher Wendepunkt ein, dann müsste sich auch die Zielsetzung des Handelns verändern im Sinne der Wegbewegung von der Lebenserhaltung hin zur Erleichterung und Begleitung des Sterbens. Das heißt, der Beginn des Sterbens beginnt hier quasi mit dem Ende aktiver medizinischer Handlungsmöglichkeiten zur Lebenserhaltung und Lebensverlängerung, über die Dauer des Sterbens ist damit noch gar nichts gesagt.

Das moderne Krankenhaus nun wiederum ist durch zwei Merkmale gekennzeichnet, wie wir es für die Mehrheit der deutschen Krankenhäuser vorfinden ${ }^{13}$ : Das Erste ist die Tatsache, dass das Krankenhaus im Gegensatz zu früher mit der Behandlung von chronischen, oft unheilbaren Krankheiten zu tun hat. So ist das Krankenhaus also durch die unheilbaren und chronischen Erkrankungen verändert worden, aber im Gegensatz dazu tendiert das Personal, vor allem Ärzte, aber auch das Pflegepersonal, immer noch dazu, die Patienten so zu behandeln, als seien sie akut krank. Das zweite Kennzeichen ist der Einfluss der Technologie auf das Krankenhaus. Medizinisch ausgefeilte Technologie hilft Leben zu verlängern, Krankheiten zu mildern und in ihrem Verlauf zu steuern. Das macht aber sowohl Patienten als auch das therapeutische Personal zunehmend abhängig von der Technologie im Verlaufe einer chronischen Erkrankung. Medizinische Spezialisierung und Technologie befruchten einander wechselseitig, sie entwickeln sich parallel und auch mit zunehmender Dynamik und machen damit die Krankenhausstruktur zunehmend komplexer. Das führt dazu, dass die Behandlung chronischer Krankheiten durch die Technologie zunehmend fragmentiert wird mit der Folge, dass die Wahrscheinlichkeit von Fehlern zunimmt und mit ihr die Notwendigkeit, diese Fehler zu kontrollieren. Vor einigen Jahrzehnten war das Krankenhaus deutlich weniger differenziert, heute ist es geradezu ein Zentrum von explodierender, sich ständig weiter ausdifferenzierender medizinischer Spezialisierung, nicht zu vergessen dabei die ebenso drastisch gestiegene Zahl und Vielfalt an Service- und Unterstützungseinheiten - wie Labore, verschiedenste Therapieverfahren, Ernährung, Reparatur, Transport - und einer sich immer stärker ausweitenden Verwaltung, die keinen direkten Einfluss auf die Arbeit einzelner Abteilungen und Stationen hat, aber indirekt über ihre Entscheidungen deren Entscheidungen und Ressourcen steuert und damit auch das medizinische und pflegerische Handeln. Das verschärft den Druck auf die Effizienz des Handelns im Sinne der Steigerung der

13 Göckenjan G \& Dreßke S (2002), vgl. Anm. 2 
Effizienz von Therapien, also der kurativen Ziele in der Behandlung von Krankheiten und der Lebenserhaltung. Folglich ist in die Definition und die Zielsetzung des Krankenhauses nicht aufgenommen die Tatsache, dass Sterben immer häufiger im Krankenhaus stattfindet, dort aber eigentlich keinen Platz hat. Die empirische Forschung über das Sterben im Krankenhaus bestätigt dieses Dilemma immer wieder: Die Begleitung Sterbender ist im Grunde kein Ziel der Institution Krankenhaus. Die Befunde der meisten Untersuchungen sind oft sehr drastisch: Der Tod wird in der Klinik oft verleugnet, die Klinik ist die Arena für den Kampf gegen den Tod, auf Sterben und Tod selbst ist man räumlich und personell nicht eingerichtet, es werden Provisorien geschaffen. Zudem gibt es für den Umgang mit Sterbenden keine klaren Handlungskonzepte und Verhaltensregeln, wie sie im Gegensatz dazu für den Umgang mit den verschiedenartigsten Erkrankungen vorhanden sind. In einer nach wie vor relevanten in ihrer Feldstudie an bundesdeutschen Krankenhäusern konstatiert Lau, „dass die von der Institution gestellten Experten zwar Strategien zur Gesundung ihrer Patienten entwickeln, dass jedoch die Pflege von einmal aufgegebenen Kranken weitgehend strategielos verläuft"14.

Es gibt eine einzige, größer angelegte empirische Untersuchung über die Sterbebedingungen in bundesdeutschen Krankenhäusern, die Gießener Studien von George und anderen. Befragt wurden mit einem überwiegend standardisierten Fragebogen Ärzte und Ärztinnen und Pflegende in vier Bundesländern in insgesamt 70 Kliniken unterschiedlicher Träger und Fachdisziplinen. Ingesamt entstand dabei alles in allem ein, wie die Autoren selber sagen, ,düsteres Gesamtbild“15: So glaubt der überwiegende Teil der Befragten, dass der Krankenhaus-Tod oftmals menschenunwürdig und belastend für Sterbende und Mitarbeiter sei. Über die Hälfte hält die bestehenden räumlichen, zeitlichen und personellen Bedingungen im Krankenhaus für ungeeignet, um Schwerstkranke ihrer Krankheit gemäß betreuen zu können. Nur $25 \%$ beschreiben gute Bedingungen. Der bestehende Personal- und Zeitmangel führt nach ihren Aussagen dazu, dass nur ein geringer Teil (28\%) glaubt, sich die notwendige Zeit für die Betreuung Sterbender nehmen zu können. Die Bedeutung eines entspannten Arbeitsklimas für die Betreuung der Patienten auf den Stationen wird durchgängig anerkannt, gleichwohl sieht sich ein Viertel mangelnder Anerkennung durch seine Berufskollegen ausgesetzt, wenn sie sich Sterbenden verstärkt widmen. Dies gilt besonders für Ärzte. Ebenso wie die Berufserfahrenen haben die auf Station mit höherer medizinischer Präsenz Arbeitenden auf das Arbeitsklima viel weniger Einfluss. Dabei werden die Sterbeumstände auf den Stationen so erlebt, dass $51 \%$ der Befragten glauben, auf diese keinen verändernden Einfluss ausüben zu können. Durch die Berufserfahrung

14 Lau EE (1975): Tod im Krankhaus. Soziologische Aspekte des Sterbens in Institutionen. Soziologische Studien, Band 5. J.P. Bachem Verlag, Köln, S. 40

15 George W, Beckmann D \& V aitl D (1989): Aktuelle empirische Daten zu den Sterbebedingungen im Krankenhaus, Psychother Psychosom Med Psychol, 39(8), 307 
sieht sich der größte Teil nur sehr unzureichend auf das Sterben im Krankenhaus vorbereitet. Nur $10 \%$ geben an, für diese Tätigkeit gut ausgebildet worden zu sein. Entgegen dem allgemeinen Vorurteil wünschen sich die Befragten (92\%) einen aktiven Einbezug der Angehörigen. Diese Bereitschaft ist so stark ausgebildet, dass die Hälfte der Befragten sich vorstellen könnte, auch Laienhelfer mit einzubeziehen. Von der zukünftigen Entwicklung der Medizintechnik versprechen sich die Befragten wenig Hilfe. Über die Hälfte (53\%) gehen in ihrer Beurteilung sogar soweit, dass aufgrund der Art, wie die Medizintechnik und Therapie angewendet wird, diese Entwicklung den sterbenden Kranken keinerlei Erleichterung verschaffen würde. Demgegenüber sind es nur zwei Prozent, die durchgängig eine helfende Wirkung attestieren. In dieses Bild reiht sich der Befund ein, dass ein großer Teil $(62 \%)$ der Befragten der Überzeugung ist, dass grundsätzlich bei hoffnungslosen Fällen zu häufig lebensverlängernde Maßnahmen ergriffen werden. Ein Drittel (35\%) geben an, dass bei Sterbenden zu selten schmerzlindernde Mittel zur Anwendung gebracht würden. Im Umgang mit der Wahrheit am Krankenbett wird eine offene Beziehung zu dem Patienten angestrebt, man spricht sich für offene Gespräche mit Patienten aus, aber man fügt hinzu, dass nur ein Drittel aller Patienten über ihre Krankheitsprognose und den wahrscheinlichen Verlauf informiert waren. Diese Diskrepanz erklärt sich nicht zuletzt dadurch, dass über die Hälfte (58\%) der Befragten nach wie vor davon ausgeht, Patienten wollten nicht über ihren Krankheitsverlauf informiert werden. Ebenfalls wird das Vorurteil noch gehegt, Kundgabe der Art der Prognose des Leidens habe einen ungünstigen Einfluss auf den weiteren Krankheitsverlauf. Beides sind nach international gesicherten Erkenntnissen falsche Annahmen. $72 \%$ der Mitarbeiter sind der Ansicht, dass ein würdevolles Sterben im Krankenhaus nicht möglich sei. So geben auch $30 \%$ der Befragten an, aufgrund ihrer Erfahrungen mehr Angst vor dem Sterben zu haben. Dabei ist insgesamt anzumerken, dass das Pflegepersonal durch seine größere Nähe zu den Patienten deren Bedürfnisse und eventuell störende Elemente stärker wahrnimmt als Ärzte und Ärztinnen.

Befragte auf Intensivstationen sehen die Sterbesituation dort durch weniger Patientennähe gekennzeichnet im Gegensatz zu den umfassender orientierten Allgemein- und Onkologie-Stationen, wo bei geringerer medizinisch-technischer Präsenz die Patienten stärker in den therapeutischen Prozess einbezogen werden. Die Autoren der Studie fordern eine Verbesserung der Ausbildung, die Vermeidung der sich extrem fatal für Sterbende auswirkenden Personalnot, offene Kommunikation aller Beteiligten, die kritischere Überprüfung eines Zugangs zum Patienten versus eines somatisch-technologischen Krankheitsmanagements und schließlich die Einführung problemorientierter Gesprächsgruppen und Fortbildungsmöglichkeiten.

Freilich muss man diese Ergebnisse differenzieren: Untersuchungen in unserer Abteilung für Medizinische Soziologie sowie andere Studien an verschiedenen Krankenhäusern, Abteilungen und Stationen machen deutlich, dass die unter- 
schiedliche Fachausrichtung entscheidend für den Umgang mit der Sterbewirklichkeit in den einzelnen Institutionen ist. So zeichnen sich vor allem onkologische Stationen durch eine stärkere Zentrierung auf beide Schwerpunkte im Umgang mit schwerstkranken Patienten aus: Auf der einen Seite wird therapeutisch solange so viel getan, wie es möglich ist, den Krankheitsverlauf positiv und lebensverlängernd zu beeinflussen, ohne dass der Patient zu stark von den Nebenwirkungen der Therapie belastet wird und ausreichend Lebensqualität hat, auf der anderen Seite wird dem Wendepunkt von kurativen Bemühungen in Richtung Sterbeverlauf und dem Umkippen der Krankheitsverlaufskurve in eine Sterbeverlaufskurve eher Rechnung getragen und entsprechend auch sehr viel stärker Palliativ-Medizin und -Pflege betrieben. Dies gilt insbesondere für rein onkologische Stationen, während eine solche Haltung auf gemischten internistischen Stationen mit onkologischen Patienten eher schwieriger zu realisieren ist. Im besonders hohen Maße gilt dieses für pädiatrisch-onkologische Stationen, wo immer auch sehr früh die Familien mit einbezogen sind, das Kindeswohl im Vordergrund steht, kurative und palliative Elemente sehr früh aufeinander bezogen werden und entsprechend auch frühzeitiger auf Sterbebegleitung zentriert wird.

Ähnliches gilt für rein geriatrische Stationen, die allerdings in dieser Form noch nicht sehr verbreitet sind, sondern die meisten Allgemein-Internistischen Stationen enthalten sehr viel Elemente einer Geriatrie, ohne dass ein rein geriatrischer Ansatz berücksichtigt wird; in den rein geriatrischen und auch gerontopsychiatrischen Einrichtungen wird sehr viel schneller und früher auf den Wendepunkt geachtet, wenn ein Krankheitsverlauf umschlägt in einen Sterbeverlauf. Es ist sicherlich kein Zufall, dass gerade diese beiden Ausrichtungen am ehesten den Zielsetzungen von Palliativ-Medizin und -Pflege und Hospiz nahe kommen, sind doch auch die Patienten oft dieselben.

Ganz anders verhält es sich bei spezialisierten Stationen wie z.B. in der Kardio-Chirurgie, hier gibt es eindeutige Kernelemente, wie den spezifischen Behandlungsauftrag des Patienten zur Operation zur Lebensrettung und -erhaltung, überwiegende Elektiveingriffe und den „Alles-oder-nichts-Charakter“ der Eingriffe, die eng zusammenhängen. Die Kardio-Chirurgie sieht bezogen auf Ärzte und Pflegepersonal ihren Auftrag in der Lebenserhaltung, das Ziel besteht vorwiegend darin, die Erkrankung zu heilen, Sterbebegleitung und Betreuung gehören nicht zum Auftrag. Die häufig schnelle Genesung der Patienten nach der Operation, die hohe Operationsrate, die beschleunigten Arbeitsabläufe und die meist verkürzten Kontaktzeiten schaffen eine ganz eigene Atmosphäre, in der sich eingehendere Beziehungen zwischen Patienten und pflegerischem und ärztlichem Personal nicht entwickeln können. Sterbende sind selten, scheinen in gewisser Weise aber auch fehl am Platze zu sein. Den Patienten in der Kardio-Chirurgie ist meistens klar, dass die in Aussicht stehende Operation ihre einzige und letzte Chance zu überleben ist. In der kurzen Zeit vor der Operation kann das Thema "Sterben“ zur Sprache kommen. Wenn die Operation beginnt, sind die Würfel schon gefallen. 
Der Kampf um Leben und Tod ist in der Kardio-Chirurgie oft ein kurzer Kampf. Erfolge und Misserfolge werden schnell offenbar. Viele Patienten gesunden schnell, aber wenn sie auf der Intensivstation zu Langzeitpatienten werden, die nicht selten sterben, gibt es nur noch wenige Möglichkeiten, sich über die richtige Behandlung mit Ärzten und Pflegepersonal auszutauschen, weil die Patienten meist nicht mehr kommunikationsfähig sind. Es entstehen also hier ganz andere Beziehungsqualitäten und Zeitstrukturen als beispielsweise in der Onkologie oder in der Palliativmedizin ${ }^{16}$.

Die Sterbewirklichkeit auf allgemein internistischen Stationen wird von dem herkömmlich gemischt internistischen Krankheitsspektrum bestimmt. Aufgenommen werden oft ältere, nicht selten vielfältig kranke Menschen mit Krebserkrankungen, geriatrischen, zum Teil psychiatrischen Krankheiten sowie auch Patienten nach Schlaganfällen. Auch Alkoholabhängige mit ihren Folgeerkrankungen werden behandelt. Der Anteil der älteren, pflegeintensiven Patienten ist hoch, die meisten Patienten sind über 60 Jahre alt. Dementsprechend sind auf solchen Stationen Sterbefälle häufig. Die Schwierigkeiten entstehen daraus, dass es sich um Mischformen handelt, in denen die Ausrichtung auf kurative Behandlung und Pflege und die daneben bestehende Aufgabe eines palliativen, auf Sterbebegleitung ausgerichteten Umgangs mit Schwerstkranken nicht miteinander vereinbart werden können. Hinzu kommt, dass diese Stationen oft personell und in ihren organisatorischen und zeitlichen Möglichkeiten nicht auf solche Aufgaben vorbereitet sind. Andererseits muss man sich auch immer wieder vor Augen führen, dass die Einführung von Palliativelementen auf solchen Stationen äußerst schwierig ist, weil es sich hier - im Vergleich zu Palliativstationen - um eine Häufung von Schwerstkranken handelt.

Unsere Untersuchung über Sterbewirklichkeiten in den verschiedenen Krankenhäusern und Stationen zeigte auch die Dilemmata über die Sterbewirklichkeit auf Intensivstationen. Es gibt Patienten, die sehr lange auf einer Intensivstation liegen, auch wenn dies relativ gesehen nicht so häufig vorkommt. Diese Patienten sind nicht unbedingt Sterbende, oft sind es Patienten, die von der Beatmungsmaschine nicht mehr loskommen. Das Dilemma der Langzeitbehandlung auf Intensivstationen speist sich dabei - bezogen auf die Sterbewirklichkeit - aus zwei Quellen: Zum einen kann sich der Genesungsprozess bei Schwerstkranken und Schwerstverletzten sehr lange hinziehen und eine lange intensivmedizinische Un-

16 Pilz S (1993): Stationen des Sterbens - Sterben auf Station. Forschungsbericht an die DFG zum Projekt: „Empirische Untersuchungen zur Praxis der passiven Sterbehilfe“ unter der Leitung von Prof. Dr. phil. H. Friedrich, Abteilung Medizinische Soziologie, Universität Göttingen; Hampel E (1992): Empirische Untersuchungen zur Praxis der passiven Sterbehilfe. Teilbericht zum Themenbereich: „Therapieverzicht“ aus der Abteilung Medizinische Soziologie, (Leitung: Prof. Dr. phil. H. Friedrïh), Universität Göttingen; Webkamp K-H (1999): Klinische Arbeitswelt und ärztliche Therapieentscheidungen zwischen Maximaltherapie und Sterbehilfe. Abschlussbericht zum DFG-Projekt: „Praxis der passiven Sterbehilfe“ aus der Abteilung für Medizinische Soziologie, (Leitung: Prof. Dr. phil. H. Friedrich), Universität Göttingen 
terstützung notwendig machen. Zum anderen können die intensiv-medizinischen Maßnahmen selbst zu einer Verlängerung des Krankheitsverlaufs führen. Selbst ein Patient mit einer unweigerlich zum Tode führenden Erkrankung kann für eine gewisse, manchmal auch sehr lange Zeit mit Hilfe medizinischer Technik am Leben bleiben. Das Problem ist das der unbestimmten Prognose, das im medizinischen Handeln immer wieder auch auftaucht und das Dilemma eines möglicherweise zu langen Therapierens impliziert. Es erhält auf den Intensivstationen ein besonderes Gewicht durch das Ausmaß der Interventionen und der damit zusammenhängenden Beeinträchtigung durch intensivmedizinische Behandlung für die Schwerstkranken, bei denen man nicht weiß, inwieweit sie schon Sterbende sind. Besonders problematisch wird hier die Situation dadurch, dass es immer wieder auch Patienten gibt und gab, die man eigentlich schon aufgegeben hatte, die die Station aber dann doch lebend verließen. Abhängig von der jeweiligen Situation entstehen bei solchen Patienten zwischen Ärzten und Pflegenden, aber auch innerhalb der jeweiligen Gruppe Konflikte und Meinungsverschiedenheiten darüber, wie lange die Intensivbehandlung gerechtfertig sei, wie lange man Patienten Belastungen aussetzen darf, wenn der Behandlungserfolg zweifelhaft ist. Die Grenzen zwischen Lebensverlängerung und Sterbeverzögerung sind oft fließend; es wird häufig konstatiert, dass Entscheidungen über eine Fortführung oder ein Absetzen der Therapie lange dauern. Das führt dann zu einem erheblichen Dilemma, weil die technischen Möglichkeiten den Rahmen der Behandlungsdauer beeinflussen, und das Einstellen von Behandlungsmaßnahmen wird dann häufig als aktive Maßnahme erlebt, die als solche bei vielen Beteiligten unter den Ärzten und Pflegenden große moralische Bedenken und Skrupel auslöst. Jede medizinische Maßnahme scheint beim Absetzen ausführlich begründet werden zu müssen. Bestimmte Maßnahmen wie Essen, Luft, Wasser, Schmerzmittel, meist auch Sedierung gehören allerdings zum Basisprogramm; sie werden auch weniger als therapeutische Maßnahmen erlebt, vielmehr als unverzichtbar zur Deckung der elementaren Grundbedürfnisse des Menschen.

Ein besonderer Aspekt des Sterbens im Krankenhaus ist die Entscheidung zum Therapieverzicht, und hier interessiert jetzt nicht die ethische oder juristische Diskussion, sondern die empirische Situation, wie sie von den insbesondere betroffenen Ärzten erlebt und wahrgenommen wird. Auch wenn es keine expliziten Regeln oder Normen für Therapieverzicht bzw. Verzicht auf lebenserhaltende Maßnahmen gibt, existieren dennoch inoffiziell allgemeine Leitwerte beim Therapieverzicht, die nicht umstritten sind. Diese Regeln beim Verzicht auf lebenserhaltende Maßnahmen umfassen vor allem folgende ${ }^{17}$ :

17 Hampel E (1992), Wehkamp K-H (1999), vgl. Anm. 16 
- Das Therapiekonzept muss ,,ausgereizt“ sein.

- Maßnahmen müssen abgesetzt werden, wenn sie „sinnlos“ und belastend sind.

- Maßnahmen dürfen nicht mehr quälen als nützen.

- Schmerztherapie muss immer gewährleistet werden.

- Flüssigkeit muss immer gegeben werden.

- Niemand soll verhungern, verbluten oder ersticken (Ausnahmen möglich).

- Grundmedikation (z.B. Insulin) soll erhalten bleiben (Ausnahmen möglich).

- Der Faktor „Lebensalter“ spielt bedingt eine Rolle.

Grundvoraussetzung der Beendigung lebenserhaltender Maßnahmen ist die als weitgehend sicher erscheinende Einsicht, dass die Maßnahmen dem Patienten nicht mehr hilfreich sind. Sie müssen als ungeeignet erscheinen, einen Krankheitsverlauf noch wesentlich zu beeinflussen, die Lebenszeit noch wesentlich zu verlängern und die Lebensqualität zu verbessern. Das Arsenal möglicher medizinischer Hilfe muss also „ausgereizt“ sein. Ganz allgemein wird das Prinzip der Leidensvermeidung oder -verminderung erkennbar: Patienten sollen nicht leiden müssen. Ein Therapieverzicht oder eine Reduktion medizinischer Maßnahmen ist dann geboten, wenn dem Patienten damit Leiden erspart oder vermindert wird, die sich entweder aus der unmittelbaren medizinischen Behandlung ergeben oder daraus, dass durch diese Maßnahmen ein Sterbeprozess qualvoll verlängert wird. Daraus folgt, dass die Schmerztherapie und selbstverständlich die Grundpflege aus dem Verzicht ausgenommen werden.

Der Behandlungsverzicht darf sich niemals auf die Dehydrierung beziehen. Die Gabe von Flüssigkeit gilt immer als geboten. Patienten dürfen nicht verdursten. Mangelnde Versorgung eines Patienten mit Flüssigkeit ist für alle befragten Ärzte unakzeptabel. Das bedeutet aber nicht, dass in jedem Fall eine Infusionsbehandlung, beispielsweise über einen zentralen Venenkatheter, als notwendig erachtet wird. Wenn absehbar ist, dass der Tod in kürzester Zeit eintritt, so kann für viele der Befragten auf die künstliche Hydrierung verzichtet werden. Patienten sollen auch nicht verhungern, d.h. die Versorgung mit kalorischer Nahrung gilt ebenfalls als ein allgemeines Grundgesetz medizinischen Handelns. Gegenüber der Flüssigkeitsversorgung steht dieser Aspekt jedoch an zweiter Stelle. An dritter Stelle werden etwa gleich häufig genannt, ein Patient solle nicht verbluten und auch nicht ersticken. Diese beiden Regeln sind gegenüber der Flüssigkeitszufuhr klar nachrangig. Hinsichtlich der Gabe von Medikamenten gehen die Ansichten auseinander. Die Mehrheit der von uns befragten Ärzte geht davon aus, dass auch Herz-Kreislauf-Medikamente und Insulin nicht abgesetzt werden sollten, auf jeden Fall dann nicht, wenn jemand bisher damit behandelt wurde.

Eine eher atmosphärisch in die Verzichtsentscheidung eingehende Überlegung bezieht sich auf das Alter des Patienten. Dabei geht es sowohl um die Frage, über welche biologischen Reserven ein Patient verfugt, als auch darum, ob jemand sein Leben bereits gelebt hat oder nicht. Beim Erreichen von hohem Alter und einer 
schweren Erkrankung wird ein Therapieverzicht eher akzeptiert. Dabei ist die Einschätzung von Bedeutung, ob der Patient sein Leben gelebt hat, was in der Regel mit dem Alter verknüpft wird. Entsprechend fällt die Entscheidung bei jüngeren Patienten und bei Kindern zum Verzicht oder das Akzeptieren der Chancenlosigkeit medizinischer Maßnahmen offensichtlich am schwersten. Ich möchte aber ausdrücklich betonen, dass bei den von uns befragten Ärzten in keinem Fall eine unmittelbare Beziehung zwischen Alter und Verzichtsentscheidung hergestellt wird. Auch psychosoziale Kriterien - wie sozialer Status, psychische Befindlichkeit, Geisteszustand, Alkoholismus, Drogenabhängigkeit - spielen keine manifeste und explizite Rolle bei der Entscheidung von Verzicht auf lebensverlängernde Maßnahmen oder Therapiefortführung. Inwieweit ökonomische Kriterien sich auf das medizinische Handeln im Krankenhaus auswirken, kann nicht beurteilt werden. Ingesamt sind bisher noch keine ernsthaften Ressourcenmängel in dem Sinne bekannt geworden, dass sie sich als ökonomische Faktoren auf einen Therapieverzicht auswirken. Allerdings wird von einer „verdeckten Rationierung" ${ }^{\text {"18 }}$ gesprochen, die sich insbesondere bemerkbar macht in Gestalt von verknappten Personalressourcen, so dass oft nicht genug medizinisches und paramedizinisches Personal vorhanden ist, um eine adäquate Behandlung oder gute Pflege und schon gar nicht eine adäquate Sterbebegleitung zu ermöglichen.

Insgesamt kann für die Sterbewirklichkeit festgehalten werden, dass es so etwas wie einen primären und sekundären Therapieverzicht gibt, wenn er auch nicht offiziell immer so definiert wird. In der klinischen Praxis haben sich unterschiedliche Strategien des Therapieverzichts oder des Verzichts auf lebensverlängernde Maßnahmen entwickelt, die sich zumeist bemerkbar machen bei der Hauptform des in Krankenhäusern vorfindlichen Sterbens, dem langen bzw. verlängerten Sterben. Berichtet wird

1. von aktivem offensichtlichem Verzicht (z.B. auf Reanimationsmaßnahmen),

2. dem langsamen „Ausschleichen“ aus der Therapie, indem z.B. Perfusoren mit Katecholaminen nicht erneuert werden, wenn sie leer sind (Katecholamine sind alle kreislaufstabilisierenden biogenen und synthetischen Amine wie Adrenalin, Noradrenalin, Dopamin und Esoprenalin). Dazu gehören auch

3. aktiv entschiedene, aber verdeckt durchgeführte Aktionen, wie nur mit halber Kraft oder nur für eine kurze Zeit reanimieren oder ganz langsam zu arbeiten. Unter Umständen wird das Pflegepersonal angewiesen, den Arzt erst dann zu rufen, wenn sich die Situation des Patienten soweit geklärt hat, dass der Arzt nicht mehr einzugreifen braucht. Entscheidungen zum Therapieverzicht verbergen sich also auch oft hinter anscheinend sehr kleinen Maßnahmen, wie z.B. Nicht-Ersetzen lebenserhaltender Therapie oder or-

18 Webkamp K-H (1999), vgl. Anm. 16 
ganisatorisch herbeigeführter Umstände, die dann einen Verzicht auf lebensrettende Maßnahmen erlauben.

4. Therapieverzicht kann aber auch aktives Beenden einer bereits laufenden Behandlung sein, indem man z.B. bei einer Beatmung die Sauerstoffzufuhr auf $21 \%$ (das entspricht dem Sauerstoffgehalt von Raumluft) erhöht, den Patienten evtl. zusätzlich sediert, damit er nicht unter Luftnot leidet und ihn sterben lässt. Oder man versucht, den Patienten, auch auf die Gefahr hin, dass er dabei stirbt, vom Respirator abzutrainieren.

5. Die Indikationsstellung: Wenn man etwas unterlassen will, was evtl. angezeigt, weil technisch möglich wäre, so sagt man, es bestehe bei dem Patienten keine Indikation mehr bzw. eine bestimmte Maßnahme sei kontraindiziert.

In der Realität sieht die Praxis von Therapieverzicht allerdings so aus, dass solche Entscheidungen eher kollegial getroffen werden, zum anderen sehr viel Unsicherheit und Angst bei den behandelnden Ärzten bestehen, solche Entscheidungen zu treffen, insgesamt eine große Verunsicherung beim Therapieverzicht anzutreffen ist.

In unseren Interviews hatten wir oft als Ergebnis, dass die meisten Ärzte doch erhebliche Hemmungen gegenüber der praktischen Durchführung von primärem, besonders aber von sekundärem Therapieverzicht hatten, weil es für sie von Schuldgefühlen begleitet wurde. Die Unterlassung, das Nichteinsetzen medizinisch-therapeutischer Optionen empfanden viele als Mitwirken am Tod oder gar als Herbeiführung des Todes des Patienten. Therapiebeendigung wird gefürchtet, weil sie als aktives Handeln zum Nachteil des Patienten interpretiert wird, während das Vorenthalten oder das Zurückhalten lebensrettender bzw. lebenserhaltender Maßnahmen als passiv und damit noch als moralisch-ethisch zulässig empfunden wird. Wer handelt, zieht Verantwortung an sich. Wer eine mögliche Handlung unterlässt, glaubt unter Umständen, dass er damit auch nicht in eine verantwortliche Rolle kommt, folglich auch nicht schuldig werden kann.

Unter den befragten Ärzten war es im Wesentlichen nur eine kleinere Gruppe, die argumentierte, dass für sie auch Therapiebeendigung zu ihrer Arbeit gehöre, wenn das, was man mit dem Beginn der medizinischen Maßnahmen angestrebt habe, sich nicht als Erfolg einstelle. Sie betonen, dass sie sich sicherer fühlen, wenn sie alle medizinischen Möglichkeiten ausprobiert haben. In dieser Gruppe sind es vor allem Ärzte, bei denen auch solche medizinischen Entscheidungen häufig in ihren Alltag gehören, weil bei ihnen Therapiemethoden-Risiken häufiger vorkommen, also vor allem Kardio- und Neurochirurgen, Anästhesisten sowie Intensivmediziner.

Insgesamt kann aber festgestellt werden, dass in bundesdeutschen Kliniken keine verbindlichen Konzepte für die Situation bestehen, ob man die Therapie fortsetzt, wann und wie Therapieverzicht entschieden und durchgeführt wird. Der Behan- 
dlungsauftrag gibt den Kliniken für diese Situation keine Differenzierung vor, und dementsprechend findet auch für Ärzte und Pflegende kein Training und keine Ausbildung im Hinblick auf das Erkennen und Bewältigen solcher Umstände statt. Sterbende, Therapieverzicht und die praktischen Folgen, nämlich die daraus resultierende Sterbebegleitung stellen im Kontext der Klinik einen „nicht definierten Rest" in der ärztlichen und pflegerischen Tätigkeit dar. Diese Leistung der Sterbebegleitung zu erbringen, wird im klinischen Arbeitszusammenhang entsprechend mehr auf einem Niveau von Freiwilligkeit, denn als Teil der professionellen Aufgabe abgehandelt. So wundert es kaum, dass es für Sterbebegleitung, inklusive Angehörigenbetreuung, vor allem unter den Ärzten und insbesondere auch ihren Vorgesetzten wenig oder gar keine soziale Unterstützung und Anerkennung gibt.

Sterben in Deutschland bedeutet, dass es sich um eine Vielzahl von Sterbewirklichkeiten handelt, die sich verteilen auf den ambulanten Bereich, in dem Hausärzte den Familien helfen, mit ihren schwerstkranken und sterbenden Angehörigen umzugehen, wobei wir interessanterweise über diesen Bereich wenige empirische Kenntnisse haben. Zu vermuten ist, dass es sich hier oft auch um eine Überforderung der Hausärzte handeln kann, die in ihrer medizinischen Ausbildung jedenfalls nicht auf Sterben und Sterbeprozesse vorbereitet worden sind. Es ist zu hoffen, dass die ambulanten palliativen und Hospiz-Dienste ausgebaut werden, die in enger Kooperation mit den Hausärzten die Familien befähigen können, ihre sterbenden Angehörigen solange wie möglich bei sich zu behalten, sofern nicht ihre subjektiven und objektiven Toleranzschwellen dabei überschritten werden.

Ich hoffe, verdeutlicht zu haben, dass es auch viele Sterbewirklichkeiten in den Alten- und Pflegeheimen gibt, wobei bekannt ist, dass man umso problematischere Situationen in Bezug auf die Sterbewirklichkeit vorfindet, je schlechter die Personalsituation und die Ressourcenzuteilung ist. In letzter Zeit sind gerade die kassenärztlichen Vereinigungen und die Krankenkassen aufgefordert worden, mehr zu tun, um geriatrische Praxisverbunde herzustellen, die vor allem verstärkt für die Pflege- und Altenheime zur Verfügung stehen. Und schließlich zeichnen sich auch Veränderungen in Bezug auf den Umgang mit Sterben und Tod im Krankenhaus $\mathrm{ab}$, denn zunehmend ist man in der gesellschaftlichen Öffentlichkeit bereit, sich mit Sterben und Tod zu beschäftigen, es werden immer mehr Vorstellungen von einem „guten“ oder „würdigen Sterben“ entwickelt, deren Leitmotive Bewusstheit, Vorbereitet-Sein, Gefasstheit und die dazu nötigen Hilfestellungen, vor allem Schmerzfreiheit und Achtung der Person durch soziale und kommunikative Angebote darstellen. Diese neuen Sterbevorstellungen und Sterbeideale scheinen auch an die Krankenhäuser höhere Anforderungen zu stellen, wenn sie auch noch im Konflikt mit den üblichen Krankenhausroutinen und -einstellungen stehen. Dennoch werden die Krankenhäuser sich stärker damit auseinandersetzen müssen, da Sterben heute selbst wichtig genommen und in ausdrücklich zugewiesenen Rollenerwartungen als eigenständiger existentieller Wert ausgearbeitet werden soll. 
Es wird interessant sein, in welchem Ausmaße gerade die Individualisierung des Sterbens und des Todes durch die Palliativ- und Hospizbewegung auch die Praxis des Krankenhauses beeinflussen und prägen werden. 


\title{
Rechtliche Typenbildung: Aktive und passive, direkte und indirekte Sterbehilfe
}

\author{
Gunnar Duttge
}

\section{Der unübersehbare Klärungsbedarf}

Mitunter ist die Verwirrung mit Händen zu greifen: Was ist erlaubt, was verboten, wenn sich der Lauf unserer Lebensuhr ihrem Ende zuneigt und andere - insbesondere behandelnder Arzt und Pflegeteam, aber auch Angehörige oder gar das Vormundschaftsgericht - auf das Geschehen Einfluss nehmen, sei es unmittelbar (i.d.R. das Leben verlängernd, aber vielleicht auch das Sterben und Leiden) oder dadurch, dass eine durchaus bestehende Behandlungsmöglichkeit nicht mehr ergriffen oder fortgesetzt wird? Dürfen schmerzlindernde und beruhigende Mittel wie Opiate oder Sedativa auch dann eingesetzt werden, wenn nicht auszuschließen oder womöglich gar nicht unwahrscheinlich ist, dass sich hiermit ein lebensverkürzender oder wenigstens das Bewusstsein auf Dauer eintrübender oder ausschaltender Effekt verbindet? Wie lange müssen oder dürfen intensivmedizinische Maßnahmen in maximalem Umfang eingesetzt werden, bzw. umgekehrt: Wer bestimmt, wann eine lebenserhaltende (und damit -verlängernde) ${ }^{1}$ Therapie redu-

1 Noch immer wird gerne zwischen „guter“ lebenserhaltender und „böser“ (bloß) lebensverlängernder Therapie differenziert. Dieser Sichtweise liegt die Vorstellung von einem schicksalhaft vorgegebenen 
ziert wird? Darf ein unerträglich leidender Patient, für den keine Aussicht auf Besserung oder gar Heilung mehr besteht, sich selbst von seinen Leiden befreien - und sich dabei ggf. auch helfen lassen, selbst von einem Arzt? Ist es andererseits aber nicht wertungswidersprüchlich, ihm im Falle seiner gänzlichen Handlungsunfähigkeit den Weg zum „Gnadentod“ zu versperren, den ein anderer (z.B. Angehöriger, aber auch ein Arzt) an seiner Statt vornehmen müsste? Wie steht es mit Organisationen, die gegen „Aufwandsentschädigung“ eine Gelegenheit zum Suizid bereitstellen und dazu ihre Hilfe anbieten? Ist solches derzeit verboten, oder sollte es wenigstens alsbald mit den Mitteln des Strafrechts untersagt werden?

Wer unbefangen auf die gegenwärtigen Debatten und Informationsangebote blickt, wird es nicht leicht haben, sich ein zutreffendes Bild von der geltenden Rechtslage und den in Aussicht genommenen Reformvorschlägen zu machen: Da berichtet etwa eine renommierte überregionale Tageszeitung ${ }^{2}$ über die bevorstehende Haftentlassung des „berühmtesten Sterbehelfers“ der USA („Dr. Death“) ${ }^{3}$ unter Beschreibung der von ihm gebauten „Selbsttötungsmaschine“ sowie jener einen Tat, deretwegen er verurteilt wurde: „Einem unheilbar erkrankten Buchhalter setzte er eigenhändig die Todesspritze. Damit war er von der passiven zur aktiven Sterbehilfe gewechselt..." Kontext begegnenden Begrifflichkeiten mit dem Hinweis, dass zur sog. passiven Sterbehilfe „im weiteren Sinne“ auch die ausreichende Gabe schmerzstillender Mittel „unter Akzeptanz eventuell auftretender Nebenwirkungen“ zähle ${ }^{6}$. In einer kürzlich veröffentlichten Pressemitteilung plädiert die Deutsche Gesellschaft für Psychiatrie, Psychotherapie und Nervenheilkunde für eine (generelle?) „Vorrangigkeit" der ärztlichen „Schutz- und Fürsorgepflicht“ beim „Umgang mit Sterbenden“ gegenüber „einer mitunter falsch verstandenen Selbstbestimmungsgläubigkeit" ${ }^{\text {"8 }}$, ohne sich daran gehindert zu sehen, in Suizidfällen bei fehlender Rettungschance zugleich für eine Nicht-Reanimation zu plädieren: „eine zwar ethisch schwierige, aber ärztliche vertretbare Position, die sich aus dem Sterben lassen als einem aktiven Handeln (?) ergeben kann..." . Selbst hochrangige Vertreter der Rechtspolitik sind vor Missverständnissen nicht gefeit: So begründet etwa die niedersächsische Jus-

(,naturgegebenen“) Verlauf voraus - eine Vorstellung, die jedenfalls heute angesichts der weit reichenden Möglichkeiten maschineller und pharmakologischer Substitution verschiedenster Organfunktionen zur Fiktion geworden ist. Infolgedessen ist heute jede lebenserhaltende zugleich eine lebensverlängernde Maßnahme!

2 F.A.Z. v. 29.10 .2005

3 Bericht über seine Verurteilung bei: Benzenhöfer, DÄ 96 (1999), A-1708 f.

4 Ricbtig: Von der bloßen Suizidbeihilfe zur aktiv(-direkten) Sterbehilfe.

5 http://www.krebsinformation.de/Fragen_und_Antworten/sterbehilfe.html (Stand: 03.09.2006)

6 Ricbtig: Indirekte Sterbehilfe.

7 Presse-Information Nr. 11 v. 27.6.2006, http://www.dgppn.de/medien/presseinfo/2006/ pdf/PM11_Suizid-NatEthikrat.pdf (Stand: 03.09.2006)

8 Nach geltendem Recht allenfalls richtig bei der aktiv-direkten, nicht aber bei der passiven und indirekten Sterbehilfe.

9 Richtig: Fallgruppe der passiven Sterbehilfe. 
Rechtliche Typenbildung

tizministerin ihr Eintreten für eine Bestrafung der geschäftsmäßigen Sterbehilfevermittlung ${ }^{10}$ mit ihrer Sorge, dass „die Grenzen zwischen der nach bisherigem Recht zulässigen passiven bzw. indirekten Sterbehilfe und der verbotenen aktiven Sterbehilfe verschwimmen“ könnten, sollte sich „die Tätigkeit professioneller

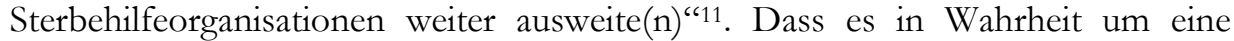
Neukriminalisierung der bisher erlaubten Suizidbeibilfe geht, erlaubt freilich schon de lege lata nur unter der Bedingung (ausnahmsweise) bestehender „Freiverantwortlichkeit“ der Selbsttötung, wird mit dem plakativen Hinweis auf die „Euthanasie-Erfahrungen des Dritten Reiches“12 selbst „grenzverschwimmend“ verdeckt. Und rätselhaft erscheint die dabei erhobene Forderung, wonach in Deutschland lediglich eine „Hilfe beim Sterben“ benötigt werde, nicht aber eine „Hilfe zum Sterben“"13: Soll künftig etwa die gleichermaßen todesursächliche und nicht weniger zielgerichtete Form der passiven Sterbehilfe ebenfalls strafbar sein - und einer intensivmedizinischen (,künstlichen“) Lebensverlängerung ad infinitum das Wort geredet werden?

Es überrascht somit nicht, wenn die hergebrachte „Sterbehilfetypologie“ neuerdings vermehrt als missverständlich angesehen und - in den Worten des früheren Bundesverfassungsrichters und heutigen Präsidenten des Deutschen Juristentages Paul Kirchbof $f^{4}$ - „neue strafrechtliche Kategorien im Umgang mit Sterbehilfe" angemahnt werden. Der Bonner Strafrechtslehrer Verrel, der das vorbereitende Gutachten zur strafrechtlichen Abteilung des diesjährigen DJT verfasst hat, beklagt ebenso wie zuvor bereits ein Arbeitskreis deutscher, österreichischer und schweizerischer Strafrechtslehrer („Alternativ-Professoren“) das Bestehen „begrifflicher Irritationen" 15 und fordert wie zuletzt auch der Nationale Ethikrat, die angestrebte „gesetzliche Absicherung“ der Grenzlinien „auf ein neues begriffliches Fundament" zu stellen ${ }^{16}$. Alle dahingehenden Stellungnahmen können sich auf empirische Studien berufen wie insbesondere auf die viel beachtete rheinlandpfälzische Ärztebefragung aus dem Jahre $2000^{17}$, die vor allem zur Abgrenzung von aktiver und passiver Sterbehilfe erhebliche Unsicherheiten zutage gefördert hat. Neuere Umfragen bestätigen dies wie auch den Umstand, dass Mediziner offenbar häufig der - juristisch irrelevanten - Differenzierung zwischen einem

10 Vgl. dazu den inzwischen auf den Weg gebrachten Gesetzentwurf des Bundesrates, BR-Drucks. $230 / 06$

11 Heister-Neumann, Recht und Politik 2006, 39, 41

12 Heister-Neumann, ebd.

13 Heister-Neumann, Recht und Politik 2006, 39

14 Berichtet u.a. in: DÄ v. 24.3.2006

15 Verrel, Patientenautonomie und Strafrecht bei der Sterbebegleitung, Gutachten C zum 66. Deutchen Juristentag 2006, C 56; ebenso Otto, NJW 2006, 2217

16 Verrel (Fn. 15), C 60; vgl. auch Nationaler Ethikrat, Selbstbestimmung und Fürsorge am Lebensende. Stellungnahme v. 13.7.2006, S. 26 ff., http://www.ethikrat.org/stellungnahmen/stellungnahmen.html (Stand: 03.09.2006); Schöch/Verrel, GA 2005, 553, 560

17 Vgl. Weber u.a., DÄ 98 (2001), A-3184 ff. 
anfänglichen (,primären“) Verzicht („withholding“) und einer erst späteren Beendigung/Reduktion der lebenserhaltenden Behandlung (,withdrawing“, „,sekundärer Therapieverzicht") eine entscheidende Bedeutung zumessen ${ }^{18}$ - mit Folgen für die tägliche Praxis ${ }^{19}$. Nicht zuletzt muss aber auch die Annahme zu denken geben, dass womöglich selbst bei Vormundschaftsrichtern/Innen „erhebliche Unsicherheiten im Hinblick auf die Unterscheidung von aktiver, passiver und indirekter Sterbehilfe bestehen“20. Was sind also die Gründe für die bisherige Typologie, was sind die materialen Wertgesichtspunkte, die jene Unterscheidungen zu tragen vermögen, und wie ist ihre Überzeugungskraft einzuschätzen - oder handelt es sich am Ende doch nur um juristisches „Kategoriengeklapper“21?

Denn eines sollte sich dabei von selbst verstehen: Es genügt keine isolierte Betrachtung einzelner Konstellationen oder Sonderfragen wie etwa jene der Patientenverfügung, auf deren gesetzesförmliche Fassung sich die aktuelle Rechtspolitik offenbar beschränken möchte 22 ; vielmehr bedarf es einer übergreifenden, konzeptionell überzeugenden, d.h. rechtsethisch fundierten Wertungsgrundlage, die das ausnahmslose Verbot einer (Fremd-)Tötung auf Verlangen ( $\int 216$ StGB) ebenso zu erklären vermag wie das (unter noch näher zu erörternden Voraussetzungen) gleichzeitige Erlaubtsein des nicht zielgerichtet (,indirekt"), des nur helfend („Beihilfe“) oder lediglich durch Unterlassen lebenserhaltender Maßnahmen („passiv“) mitbewirkten Zu-Tode-Bringens. Die höchstrichterliche Rechtsprechung hat trotz Erhellung einzelner Aspekte ${ }^{23}$ erklärtermaßen bisher kein in sich geschlossenes, in jeder Hinsicht widerspruchfreies und sachgerechtes System der unterschiedlichen Fallgruppen hervorgebracht ${ }^{24}$. Daraus speist sich, die zögerliche

18 Nachweise bei Verrel (Fn.15), C 54

19 So lässt sich belegen, dass - dem Grundanliegen der Lebenserhaltung zuwiderlaufend - eher auf lebenserhaltende Maßnahmen von vornherein verzichtet wird („Unterlassen“), als selbige erst im weiteren Verlauf (vermeintlich stets ,aktiv“) zurückzunehmen, vgl. die Ergebnisse der medizinsoziologischen Studie von Hampel, Therapieverzicht, 1997, S. 111 ff., im Internet unter http://webdoc. sub.gwdg.de/diss/1997/hampel/inhalt.htm (Stand: 03.09.2006); ebenso die Ergebnisse der schweizerischen Todesfallstudie, vgl. Bosshard u.a., Begrenzung lebenserhaltender Maßnahmen bei Patienten am Lebensende in der deutschsprachigen Schweiz - Resultate einer Todesfallstudie, DMW 130 (2005), 2887, 2889.

20 Simon/Tietze/Nickel/van Oorschot, MedR 2004, 303 ff.; zur Bedeutsamkeit medizinischen Wissens am Beispiel des ,apallischen Syndroms“ erhellend Schäfer, in: Höfling (Hrsg.), Das sog. Wachkoma. Rechtliche, medizinische und ethische Aspekte, 2005, S. 11, 17 ff., 27

21 Engisch, in: Jescheck (Hrsg.), Dreher-FS, 1977, 309, 315

22 Siehe etwa Zypries, Interview in der Berliner Zeitung v. 21.1.2006.

23 Aus der höchstrichterlichen Rechtsprechung: BGHSt 32, 367 ff. (Rettungspflicht bei freiverantwortlicher Selbsttötung); BGHSt 37, 376 ff. (Verbot der gezielten aktiven Tötung); BGHSt 40, 257 ff. (Behandlungsabbruch); BGHSt 42, 301 ff. (erlaubte Schmerzmedikation); siehe weiter OLG München NJW 1987, 2940 ff. (straflose Beihilfe zur freiverantwortlichen Selbsttötung); LG Ravensburg NStZ 1987, 229 (verlangter Behandlungsabbruch); näher zu den Entwicklungen der letzten 20 Jahre: Verrel (Fn. 15), C 13 ff.

24 So mit Bezug auf die Beteiligung Dritter an den verschiedenen Stadien eines freiverantwortlich ins Werk gesetzten Suizids explizit BGHSt 32, 367, 371; vgl. auch die kritische Sicht des Bundesgerichtshofs in Zivilsachen, BGH NJW 2005, 2385, 2386 
Rechtliche Typenbildung

Haltung der aktuellen Rechtspolitik nicht mehr länger duldsam hinnehmend, die Forderung der Strafrechtswissenschaft nach Herbeiführung einer den Gesamtkomplex der sog. „Sterbehilfe“ umfassenden Rechtssicherheit. Mit Recht betonen die Verfasser des im vergangenen Jahr vorgelegten „Alternativ-Entwurfs Sterbebegleitung“"25: „Die im Strafgesetzbuch enthaltenen Vorschriften über den Lebensschutz (Tötungsdelikte) entstammen einer Zeit, in der man sich noch keine Gedanken darüber gemacht hat, dass es einst möglich sein würde, dem Schicksal einer tödlichen Erkrankung entgegenzutreten und dabei einzelne Körperfunktionen auch über lange Zeit künstlich aufrechtzuerhalten“; es fehlen mithin bis heute „gesetzliche Regelungen darüber, in welchen Fällen es ausnahmsweise zulässig sein könnte, den Arzt von seiner Pflicht zur Lebenserhaltung zu entbinden oder ihm gar lebensverkürzende Eingriffe zu gestatten“26. Die große Schwierigkeit besteht allerdings darin: Jeder Vorschlag einer Gesamtregelung muss sich am skizzierten Leitbild einer übergreifenden Wertungskonsistenz messen lassen ${ }^{27}$.

\section{Die geltende Rechtslage und ihre Gründe}

1. Ausgangspunkt sämtlicher ins Grundsätzliche zielenden Überlegungen muss die Frage sein, ob es dem ohne sein Zutun in die Welt ,geworfenen“ Menschen als Individuum überhaupt „,erlaubt“ ist, über das eigene Leben frei zu verfügen - und zwar nicht nur hinsichtlich seiner inhaltlichen Ausgestaltung („Wie soll ich leben“?), sondern auch und vor allem mit Blick auf das „Ob“, also bzgl. einer Fortdauer oder Preisgabe des (eigenen?) Lebens. Denn wenn sich schon im Rahmen solcher Selbstbezüglichkeit (d.h. außerhalb eines Rechtsverhältnisses) ${ }^{28}$ begründete Vorbehalte formulieren lassen, wie sollte dann gerechtfertigt werden können, das Leben eines anderen zu beenden oder daran wenigstens mitzuwirken. Dass die Bedenken und Einsprüche gegen eine „Liberalisierung“ der Sterbehilfe noch immer teilweise durch eine zumindest ambivalente, mitunter sogar eindeutig missbilligende Haltung zum Suizid bedingt sind, wird schlaglichtartig deutlich, wenn der Bundesgerichtshof in Strafsachen noch im Jahre 2001 wie selbstverständlich eine (angeblich) von der Rechtsordnung vorgegebene „Rechtswidrigkeit“ der Selbsttötung behauptet ${ }^{29}$ und zum Beleg auf ein Präjudiz aus dem Jahre 1954 verweist, in dem es nicht weniger apodiktisch heißt: Es ist das „Sittengesetz“, das ,jeden Selbstmord - von äußersten Ausnahmefällen vielleicht abgesehen - streng missbilligt, da

25 Siehe bereits o. bei Fn. 15

26 Schöch/Verrel, GA 2005, 553, 555

27 Zu den kritischen Punkten des AE-StB 2005 vgl. näher Duttge, GA 2006, 573 ff.

28 Häufig wird übersehen, dass selbst die bei Kant gegen den „Selbstmord“ bemühte „Pflicht gegen sich selbst" lediglich in der Tugend-, nicht jedoch in der Rechtslehre behandelt wird; zu den Bedenken gegen die kantischen Argumente vgl. die erhellende Analyse von Wittwer, Kant-Studien 92 (2001), $180 \mathrm{ff}$.

29 BGH NStZ 2001, 324, 326 m. abl. Anm. Duttge, NStZ 2001, 546 ff. 
niemand selbstherrlich über sein eigenes Leben verfügen und sich den Tod geben darf ${ }^{4}$ “30.

Versuche einer solcherart ,naturrechtlichen“"Verurteilung des Freitodes haben in der Philosophiegeschichte bekanntlich eine lange Tradition ${ }^{31}$. Sie müssen freilich spätestens seit Humes nachdrücklichem Plädoyer für die „Wiedereinsetzung“ des Menschen in seine „angeborene Freiheit" ${ }^{“ 32}$ als widerlegt angesehen werden. Dies gilt insbesondere auch für die mitunter noch immer begegnende „Geschenk"-Metapher, wonach also - mit den Worten der päpstlichen Kongregation für die Glaubenslehre ${ }^{33}$ - das menschliche Leben ein „Geschenk der Liebe Gottes“" sei, so dass die Selbsttötung eine „Zurückweisung der Oberherrschaft Gottes und seiner liebenden Vorsehung" bedeute ${ }^{34}$ - oder wie es Thomas von Aquin noch etwas deutlicher formuliert hat: eine „Sünde gegen Gott“, ebenso groß wie jene, die geschieht, wenn jemand „einen fremden Sklaven tötet“"35. Diese wenigstens dem Namen nach in der (strafrechtlichen) Lehre vom „absoluten Lebensschutz“36 augenscheinlich fortwirkende Vorstellung von einer „Heiligkeit“ menschlichen Lebens erhöht ähnlich Schweitzers „Ethik der Ehrfurcht vor dem Leben“37 den Lebensschutz zu einem weltanschaulich fundierten Leitprinzip a priori, das mit einer säkularen, gegenüber allen Rechtsgenossen mit Rechtszwang ausgestatteten Rechtsordnung nicht verträglich ist: Denn wer die religiöse Perspektive von einer tieferen Verwurzelung des Menschen innerhalb einer göttlichen Schöpfungsordnung nicht teilt, kann sich an eine hieraus begründete Verpflichtung zum Weiterleben auch nicht gebunden sehen ${ }^{38}$. Ohnehin erkennt die geltende Rechtsordnung, die sich der Religionsfreiheit und der weltanschaulichen Neutralität der verfassten Staatsgewalt verpflichtet weiß (vgl. Art. 4 I, 140 GG i.V.m. Art. 137 I WRV), keineswegs ein ,absolutes“39 Lebensrecht an; vielmehr lässt das (Grund-)Recht (!) auf Leben aus Art. 2 II GG (im Unterschied zu Art. 1 I GG) sogar hoheitliche Eingriffe ,auf Grund eines Gesetzes“ zu (S. 3), was für die Anerkennung des (in sei-

\footnotetext{
30 BGHSt 6, 147, 153

31 Dazu die lesenswerte Monographie von Decher, Die Signatur der Freiheit, 1999 (dazu Bspr. Duttge, GA 2003, 179 ff.)

32 Vgl. Hume, Über Selbstmord, in: Kreimendahl (Hrsg.), Die Naturgeschichte der Religion, 1984, S. $89 \mathrm{ff}$.

33 Erklärung zur Euthanasie v. 05.05.1980, Ziff. I. http://www.vatican.va/roman_curia/congregations/cfaith/documents/rc_con_cfaith_doc_19800505_euthanasia_ge.html (Stand: 3.09.2006)

34 Hiergegen protestierend Schreiber, in: Thiele (Hrsg.), Aktive und Passive Sterbehilfe. Medizinische, rechtswissenschaftliche und philosophische Aspekte, 2005, S. 124

35 Thomas von Aquin, Summa Theologica, Bd. 18, 64. Untersuchung, 5. Artikel, 1987

36 Vgl. Gropp, in: Kreutzer (Hrsg.), Brauneck-Ehreng. 1999, S. 285, 290; Wessels/Hettinger, Strafrecht Besonderer Teil. Bd. 1. 29. Aufl. 2005, Rn 2.

37 Schweitzer, Kultur und Ethik, 1923, insbes. Kap. XXI (S. 328 ff.).

38 Duttge, ZfL 2004, 30, 32; Quante, Ethik in der Medizin 1998, 206, 216.

39 Zum „Absoluten im Recht“vgl. Duttge/Löwe, Jahrbuch für Recht und Ethik 2006 (im Erscheinen).
} 
nem Kernbereich unverfügbaren) Notwehrrechts („Ur-Recht“) ${ }^{40}$ geradezu conditio sine qua non ist. In diesem Sinne sieht auch die Europäische Menschenrechtskonvention eine Ausnahme vom Tötungsverbot vor (Art. 2 II lit. a EMRK).

Dass hier nicht mehr vom Schutz menschlichen Lebens, sondern vielmehr von einem Recht des einzelnen Subjekts die Rede ist, zeigt die fundamentale Divergenz der Betrachtungsweise. Denn einer Berechtigung ist stets die Freiheit der Wahl immanent: „Wer nicht zwischen Alternativen wählen darf, dem ist in Wahrheit gar kein Recht gegeben“41. Wenn Art. 2 II GG daher ein Recht auf Erhaltung des eigenen Lebens verbürgt, so schließt diese grundrechtliche Garantie denknotwendig die negative Freiheit in sich ein, mithin den Willen zum Nichtgebrauch des Rechts zum Leben, d.h. also zum Tode. Anders wäre etwa das im Rechtsdiskurs heute ganz und gar unstreitige ${ }^{42}$ und wohl inzwischen ebenso im Bereich der Medizin (selbst bei vitaler Indikation) weithin akzeptierte Vetorecht des Patienten gegenüber der Vornahme eines ärztlichen Heileingriffs - eben unter Berufung auf sein „Selbstbestimmungsrecht“ (Fall der einverständlichen „passiven Sterbehil$\left.\mathrm{fe}^{\mathrm{c}}\right)^{43}$ - überhaupt nicht begründbar. Dies ist bekanntlich jedoch umstritten, ${ }^{44}$ letztlich vor allem der Sorge wegen, dass die Anerkennung einer solchen Berechtigung womöglich entsprechende Pflichten der Hoheitsgewalt nach sich ziehen könnte ${ }^{45}$. Die gegenwärtige Situation beim (steuerfinanzierten!) ${ }^{46}$ Schwangerschaftsabbruch legt eine solche Schlussfolgerung durchaus nahe. Zur verallgemeinerungsfähigen These kann diese aber nicht befestigt werden, da sich dem Sozialstaatsprinzip (Art. 20 I, 28 I GG) keineswegs die Pflicht zu vollumfänglicher Förderung jedweder Willensbestimmung entnehmen lässt und noch sehr die Frage wäre, ob das hiernach nur mehr gewährleistete „Existenzminimum“47 staatliche Hilfen gerade zur Herbeiführung des Todes nach sich zieht. Wie im Falle der gegenwärtig diskutierten Kriminalisierung der organisierten Sterbehilfevermitt-

40 So etwa Engisch, Auf der Suche nach der Gerechtigkeit, 1971, S. 275 f.; geschichtliche Hinweise bei Leipziger Kommentar zum Strafgesetzbuch (LK)/Spendel (Hrsg.: Jähnke), 11. Aufl. 2003, \32 Rn $1 \mathrm{ff}$.

41 Duttge, ZfL 2004, 30, 32

42 Grdl. BGHSt 11, $111 \mathrm{ff}$.

43 Dazu näher u. 4

44 Siehe die Nachweise bei Verrel (Fn. 15), C 75 Fn. 337; zuletzt abl. Hillgruber, ZfL 2006, Heft 3 (im Erscheinen); Rosenau, in: Heun/Honecker u.a. (Hrsg.), Evangelisches Staatslexikon, 2006, Sp. 2369, der jedoch über den (Um-)Weg des Art. 1 I GG ein „Recht auf einen würdevollen Tod“ herleitet.

45 Vgl. Beckmann, in: Beckmann/Löhr/Schätzle (Hrsg.), Sterben in Würde, 2004, S. 224: „,...dann wäre auch ärztliche Hilfe geboten"; dies ist jedoch bereits wegen der Eigenwertigkeit des ärztlichen Ethos nicht zu befürchten, s.u. bei Fn. 66.

46 Die Gesundheitsminister der Länder sind offenbar inzwischen um eine Reduzierung der Kosten bemüht, berichtet in: KNA v. 29.6.2006.

47 Vgl. etwa Benda, in: Benda/Maihofer/Vogel (Hrsg.), Handbuch des Verfassungsrechts der Bundesrepublik, 1994, \17 Rn 116; zur Notwendigkeit restriktiver Auslegung siehe Her₹og, in: Maunz/ Dürig (Hrsg.), Grundgesetz, 2005, Art. 20 VIII Rn 24 ff. 
lung ${ }^{48}$ sollte stärker darauf geachtet werden, dass die Verantwortungsbereiche nicht miteinander vermischt werden: Wer nicht mit Rechtszwang interveniert, erkennt zwar den Raum freiheitlicher Betätigung als solchen, nicht aber zugleich die Richtung des geübten Freiheitsgebrauchs an. Läge es anders, bliebe von solcher „Freiheit“ in Wahrheit nichts übrig; selbige stünde vielmehr von vornherein unter dem Vorbehalt vorgefertigter Bilder (von Staats wegen) gesollter „Glückse-

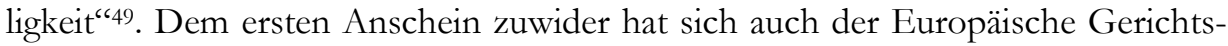
hof für Menschenrechte im Fall Diane Pretty ${ }^{50}$ - in einer Konstellation verlangter Fremdtötung, nicht eines beabsichtigten Suizids - für das (mögliche) Bestehen eines Selbstbestimmungsrechts zum Tode hin ausgesprochen, freilich nicht aus Art. 2, sondern aus Art. 8 EMRK („Recht auf Achtung des Privatlebens“) begründet. Die im Kontext des Lebensrechts (Art. 2 EMRK) zum Vorschein kommende Haltung eines verabsolutierten Lebensschutzes (,sanctity of life“) ${ }^{51}$ ist daher nur eine scheinbare, die sich erst aus der unterschiedlichen Reichweite der zwischen beiden Rechten bestehenden Beschränkungsmöglichkeiten erklärt ${ }^{52}$.

Was somit nach alledem nur zweifelhaft sein kann, ist nicht etwa das Bestehen eines selbst gegen das Weiterleben gerichteten Selbstbestimmungsrechts, sondern vielmehr die hierzu nötige Befähigung zum „freiheitlichen“ Gebrauch im jeweiligen lebensweltlichen Kontext. Der auf Erhaltung des Selbst gerichtete „Naturtrieb“ spricht doch prima vista gegen eine „wohlüberlegte“, begründete „Selbstbestimmung zum Tode“ hin. Die eingangs schon erwähnte Stellungnahme der Deutschen Gesellschaft für Psychiatrie, Psychotherapie und Nervenheilkunde ${ }^{53}$ beruft sich in ihrem Plädoyer für eine Stärkung der ärztlichen Fürsorgepflicht daher nicht ohne Grund auf den hohen Anteil an Suizidenten mit zum Teil massiven psychosozialen Beeinträchtigungen. Eine erst vor wenigen Jahren im Auftrag des Bundesministeriums für Familie, Senioren, Frauen und Jugend erstellte Expertise spricht - eine Mehrzahl empirischer Studien jüngeren Datums zusammenfassend - von einer nachweislich „häufigen“ Verbindung des Suizids mit psychischen Erkrankungen (insbesondere Depressionen), ohne dass diese allerdings als alleinige Ursachen gelten können ${ }^{54}$. So bleibt in einem häufiger auf wenigstens $5 \%$ aller Fälle geschätzten

48 Siehe bereits o. bei Fn. 10
49 Zur Renaissance, „perfektionistischer“ Positionen vgl. Pauer-Studer, in: Schmücker/Steinvorth
(Hrsg.), Gerechtigkeit und Politik. Philosophisches Perspektiven, 2002, S. 77 ff.
50 EGMR NJW 2002, 2851 ff.
51 Ebd., 2852 Ziff. 39. Krit. wie hier auch Verrel (Fn. 15), C 76 m. Fn. 342
52 Dazu näher Duttge, KHuka - Archiv des öffentlichen Rechts 2005, 174 ff. - Der EGMR hat ein Recht auf Fremdtötung in der Sache unter Hinweis auf den Bestand der Rechtsordnung („,demokratische Gesellschaft") abgelehnt.

53 Siehe o. Fn. 7: „In einem solchen Fall kann nicht von einer selbstbestimmten Entscheidung gesprochen werden, sondern die adäquate Behandlung der Erkrankung muss erst die Fähigkeit zu einer selbstbestimmten Urteilsfähigkeit wiederherstellen“.

54 Vgl. Erlemeier, in: Bundesministerium für Familie, Senioren, Frauen und Jugend (Hrsg.), S. 65 ff., 86; ähnlich Netə, in: Wolfslast/Schmidt (Hrsg.), Suizid und Suizidversuch. Ethische und rechtliche Herausforderung im klinischen Alltag, 2005, S. 81, 82 f. sowie kürzlich auch die Stellungnahme der 
Umfang55 sehr wohl die Möglichkeit eines nicht krankheitsbedingten, „reiflich überlegten“ Suizids einzukalkulieren, insbesondere dort, „wo unheilbare körperliche Erkrankung, Mangel aller Mittel und völlige Isolierung in der Welt zusammenkommen "56. Dem entspricht in der (Straf-)Rechtswissenschaft ungeachtet aller im Detail bestehenden rechtsdogmatischen Meinungsverschiedenheiten die Unterscheidung zwischen dem (im Zweifel - zugunsten des Lebens - anzunehmenden) ${ }^{57}$ Regelfall eines (nicht freiverantwortlichen) „Appell-“58 und dem Ausnahmefall eines „Bilanz-Suizids“. Erweisen sich also nach menschlichem Ermessen Zweifel an der erforderlichen „Freiverantwortlichkeit“ ausnahmsweise als unbegründet, was als Option anzuerkennen für all jene unausweichlich ist, die Suizidwillige nicht pauschaliter „psychiatrisieren“ wollen, so lassen sich insoweit gegen eine demgemäß „selbstbestimmte“ Selbsttötung keine begründeten Bedenken mehr erheben.

Das in solchen Fällen zum Tragen kommende „Autonomieprinzip“ sperrt nach dem Willen des deutschen Gesetzgebers wegen Vorliegens einer erlaubten Tat

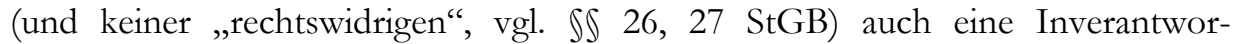
tungnahme hieran bloß „teilnehmender" Personen (also von Gehilfen und Anstiftern, $\ 28$ II StGB). Strafrechtsdogmatisch zeigt sich darin das sog. „Akzessorietätsprinzip“, das den Schluss auf das Vorliegen einer strafbaren „Teilnahme“ verbietet, wo die helfende oder motivierende (den „Tatentschluss“ hervorrufende) Beteiligung auf eine „Haupttat“ zielt, die selbst kein Unrecht darstellt (weshalb die Qualifizierung der Selbsttötung - allenfalls ein Verstoß gegen das „Sittengesetz“ oder auch gegen die Rechtsordnung - von erheblicher Relevanz ist) ${ }^{59}$. Ein Blick über die Grenzen des nationalen Rechts hinweg erweist jedoch, dass die Erweiterung des Freiraums auf Anstifter und Gehilfen nicht überall geteilt wird: ${ }^{60}$ Europaweit zeigt sich in der Mehrzahl sogar ein striktes Verbot der Suizidteilnahme, das etwa in Österreich (vgl. \78 ö-StGB) selbst im Strafrahmen zu einer Gleichstellung mit der (vom Täter „beherrschten“) Tötung auf Verlangen geführt hat. Das niederländische Recht postuliert mit der Durchbrechung des grundsätzlich

Nationalen Ethikkeommission der Schweiz im Bereich Humanmedizin Nr. 9/2005, S. 27: „Meist ist aus psychiatrischer Sicht die Freiheit der Entscheidung bei Suizidalität nur bedingt vorhanden“, sowie ebd., S. 28: psychische Erkrankungen als „wichtigster Risikofaktor“.

55 Z.B.: Kutzer, in: Wolfslast/Schmidt (Fn. 54), S. 181, 186; deutlich höhere Schätzung bei Held, in: Brudermüller/Marx u.a. (Hrsg.), Suizid und Sterbehilfe, 2003, S. 165: „bei $30 \%$ auch keine hypothetische Zuständigkeit der Psychiatrie“.

56 Jaspers, Philosophie, Bd. II, 1956, S. 308 f.: Hier „kann in höchster Klarheit ohne Nihilismus das eigene Dasein nicht überhaupt, sondern das, was jetzt noch bleiben könnte, negiert werden“"

57 Etwa Kutzer, in: Wolfslast/Schmidt (Fn. 54), S. 181, 189; Schöch, ebd., S. 163, 169: strafrechtliche Hilfspflicht bereits dann, wenn Freiverantwortlichkeit „offensichtlich zweifelhaft“ ist.

58 Vgl. etwa Duttge u.a., Preis der Freiheit, 2006, S. 96 f.

59 Dazu o. bei Fn. 29

60 Auch in Deutschland wird z.T. die Einführung einer eigenständigen Teilnehmerbestrafung für zulässig gehalten, vgl. Hillgruber, ZfL 2006, Heft 3 (im Erscheinen), Fn. 55; Ingelfinger, Grundlagen und Grenzbereiche des Tötungsverbotes, 2004, S. 224 
bestehenden Verbots bei Einhaltung eben jener „Sorgfaltskriterien“61, die auch die Freigabe der aktiv-direkten Sterbehilfe kontrollierbar machen soll, eine Gleichstellung anderer Art (vgl. Art. 293, 294 ndl-StGB), freilich nur mit der Beihilfe, während die Anstiftung ausnahmslos verboten geblieben ist und daher wertungswidersprüchlich strenger beurteilt wird als die (täterschaftliche) Tötung auf Verlangen. Dagegen sanktioniert das Strafrecht der Schweiz die Teilnahme am Suizid nur unter der zusätzlichen Voraussetzung des Bestehens ,,selbstsüchtiger Beweggründe“ (Art. 115 schw-StGB), was auf den ersten Blick wie ein sympathisch-pragmatischer Mittelweg erscheint, bei näherem Hinsehen sich jedoch auf dem Boden des Autonomiegedankens als bedenkliches Gesinnungsstrafrecht erweist, mit dem sich überdies - wie die Praxis gezeigt hat - das Treiben der bekannten „Sterbehilfeorganisationen" keineswegs eindämmen lässt ${ }^{62}$. Wie die Nationale Ethikekommission der Schweiz im Bereich Humanmedizin in ihrem Bericht vom April des vergangenen Jahres zu Recht herausgestrichen hat, sollte dieses Problem daher besser keine Frage des Strafrechts sein, sondern vielmehr die einer effektiven staatlichen Aufsicht ${ }^{63}$. Entsprechende Kriminalisierungsbestrebungen in Deutschland, insbesondere anlässlich der Eröffnung eines Büros des Vereins „Dignitas“ in Hannover ${ }^{64}$, mit allgemeinerem Bezug aber auch enthalten in zentralen rechtspolitischen Dokumenten der jüngsten Vergangenheit (Bioethik-Kommission Rheinland-Pfalz; Alternativ-Entwurf Sterbebegleitung, Nationaler Ethikrat) ${ }^{65}$, sollten demzufolge auch wegen der nicht überschaubaren Folgewirkungen auf Bereiche jenseits des unmittelbar Geregelten („Einheit der Rechtsordnung“) - noch einmal in Ruhe überdacht werden.

Selbst dort, wo sich ein Arzt als Gehilfe oder Anstifter an einer „freiverantwortlichen" Selbsttötung beteiligt ${ }^{66}$, kennt das deutsche Recht keine straf- oder zivilrechtliche Sanktionsnorm. Allerdings betonen die „Grundsätze der Bundesärztekammer zur ärztlichen Sterbebegleitung“ v. 7. Mai 2004 die ausnahmslose Standeswidrigkeit selbst einer nur randständigen Beteiligung des Arztes an einer Selbsttötung ${ }^{67}$, weil - wie der Präsident der Bundesärztekammer jüngst zu bedenken gegeben hat - „,mit der Methode des assistierten Suizids ... das Verbot der

61 Deutsche Fassung abrufbar unter: www.dgpalliativmedizin.de (Stand: 03.09.2006)

62 So explizit der Züricher Staatsanwalt Brunner in seinem Vortrag anlässlich der Fachtagung der niedersächsischen CDU-Fraktion v. 25.11.2005.

63 Vgl. Nationale Ethikkeommission (Fn. 54), S. 63 und 70; dazu näher Duttge u.a. (Fn. 58), S. 99 ff.; zust. Schreiber, NStZ 2006, 473, 478

64 Siehe o. bei Fn. 10

65 Bioethik-Kommission des Landes Rheinland-Pfaly, Sterbehilfe und Sterbebegleitung. Ethische, rechtliche und medizinische Bewertung des Spannungsverhältnisses zwischen ärztlicher Lebenserhaltungspflicht und Selbstbestimmung des Patienten, 2004, Empfehlung 3, Absatz 2 (S. 133): im Falle der „Mitwirkung bei einer Selbsttötung ... aus Gewinnsucht"; Schöch/Verrel, GA 2005, 553, 582 (m. krit. Bspr. Duttge, GA 2006, 573); Nationaler Ethikrat (Fn. 16), S. 49 f.

66 Wie im „Fall Hackethal“, vgl. OLG München NJW 1987, 2940 ff.

67 Bundesärztekammer, Grundsätze zur ärztlichen Sterbebegleitung v. 7.5.2004, www.bundesaerztekammer.de/30/Richtlinien/ Empfidx/Sterbebegl2004.pdf (Stand: 03.09.2006), Präambel 
Tötung auf Verlangen umgangen werden“ könne ${ }^{68}$. Hinter dieser Sorge steht letztlich aber vor allem ein spezifisches Selbstverständnis, d.h. ein ,berufsethisches Leitbild“, das von der ärztlichen Aufgabe zur „Vorbeugung und Heilung von Krankheiten sowie (der) Linderung von Leiden" geprägt ist ${ }^{69}$. In diesem Lichte kann die Suizidassistenz ,nicht Teil der ärztlichen Tätigkeit sein“70 und wird deshalb auch vom Weltärztebund ${ }^{1}$ verurteilt, mag sie vereinzelt in standesrechtlichen Stellungnahmen wie etwa der Schweiz ${ }^{72}$ oder Großbritanniens ${ }^{73}$ auch ,im Einzelfall“ als akzeptierbar angesehen werden. Da Arztethik und Recht als je eigenständige normative Kreise zu begreifen sind, die sich zwar im Überschneidungsbereich ihres Gegenstandes wechselseitig befruchten (können), ihrer Eigenständigkeit dadurch aber nicht verlustig gehen, darf die deutsche Ärzteschaft in gleicher Weise die Unvereinbarkeit der Suizidbeihilfe mit dem ärztlichen Ethos behaupten, wie es dem Recht unbenommen bleibt, den Unrechtscharakter selbst des ärætlich assistierten Suizids unter Verweis auf das „Selbstbestimmungsrecht“ des Suizidenten in Abrede zu stellen ${ }^{74}$. Soweit der AE-StB 2005 eine „Auflockerung“ des ärztlichen Standesrechts anmahnt in Richtung einer Ausnahmeklausel für Fälle, in denen „nach Ausschöpfung aller therapeutischen Möglichkeiten“ die Suizidbeihilfe der „Abwendung eines unerträglichen und unheilbaren Leidens“" dient ${ }^{75}$, liegt darin eine „Kompetenzanmaßung“, die auch aus Sachgründen keine Zustimmung verdient: Denn in der vorgeschlagenen Fassung („Aussichtslosigkeit“, „,unerträgliches Leiden") ließe sich die Regelung leicht als generalisierbarer Ausdruck des Vorliegens „unwertigen“ menschlichen Lebens (miss-) verstehen, „mithin - ohne Änderung der gewählten Formulierung - gleichsam als ,trojanisches Pferd' wirkend für eine logisch kaum mehr abweisbare Übertragung auf den Bereich der Fremdtötung (auf Verlangen) ${ }^{676}$.

2. Die Straflosigkeit der Mitwirkung an einer vom Sterbewilligen veranlassten Todesherbeiführung steht allerdings unter einem doppelten Vorbehalt: Zum einen bedarf es der hinreichenden „Freiverantwortlichkeit““ des Zu-Tode-Kommenden,

68 Pressemitteilung v. 17.7.2006, www.bundesaerztekammer.de (Stand: 03.09.2006)

69 Beschluss VII., 16. des 109. Deutschen Ärztetages 2006, www.bundesaerztekammer.de/30/ Aerztetag/109_DAET/03Beschluss/Top07/16/index.html (Stand: 03.09.2006)

70 So explizit die Empfehlungen der Schweizerischen Akademie der Wissenschaften. Im Internet unter www.klinikum-nuernberg.de/klinikum/kliniken/zd/cekib/leistungen/ethik/vortraege/

Prof_Reiter-Theil_Suicis_und_Ethik.pdf (Stand: 04.09.2006)

71 Abrufbar unter: www.wma.net/e/policy/e13b.htm (Stand: 03.09.2006)

72 Ebd.

73 Zit. nach: Schildmann/Vollmann, DMW 131 (2006), 1405, 1408

74 Solange der Gesetzgeber die Missachtung des ärztlichen Pflichtenkreises als solche (sog. „,kontraindizierter Heileingriff" $)$ nicht für strafwürdig und -bedürftig hält. De lege ferenda bleibt jedoch überdenkenswert, ob das Vertrauen in eine Ärzteschaft, die dem Leben verpflichtet ist, nicht auch als hochrangiger Belang der Allgemeinheit begriffen werden muss.

75 Schöch/Verrel, GA 2005, 580 f. und 586

76 Duttge, GA 2006, 573, 585 
was sich nach den bereits vorstehend skizzierten Erkenntnissen der Psychiatrie ${ }^{77}$ nur in seltenen Ausnahmefällen annehmen lässt - sofern sich die Prüfung von Rechts wegen nicht in der Feststellung des Nichtvorliegens jugendlicher Unreife, eines Zustands manifester geistiger Erkrankung, einer schweren seelischen Störung oder einer gravierenden Notstandslage (angelehnt an $\iint$ 19, 20, 35 StGB) erschöpft („Exkulpationslösung ${ }^{6}{ }^{78}$, sondern - richtigerweise - weitergehend der positive Nachweis hinreichender Einsichts- und Urteilsfähigkeit bzw. der nötigen „Ernstlichkeit“ bzgl. der Irreversibilität des Selbsttötungsentschlusses (nach Maßgabe der rechtfertigenden Einwilligung bzw. des \ 216 StGB, sog. „Einwilligungslösung $\left.{ }^{6}\right)^{79}$ verlangt wird. Fehlt es daran, so wird sich das äußerlich als straflose Teilnahme (Beihilfe, Anstiftung) an einer Selbsttötung erscheinende Geschehen bei wertender Betrachtung häufig als Fall einer in mittelbarer Täterschaft ( $\int 25 \mathrm{I}$ Alt. 2 StGB) begangenen Fremdtötung darstellen, weil der vermeintliche „Teilnehmer" das Suizidgeschehen dann in Wahrheit meist als „Zentralgestalt ... steuern“80 wird. Umso mehr wächst diesem - zweitens - die (strafrechtliche) „Verantwortung“ für den herbeigeführten Erfolg zu, wenn er die Tötung (in zutreffender Kenntnis des Kausalverlaufs ohne „tatherrschaftsbegründende“ Mitwirkung des „Opfers“") ${ }^{81}$ selbst vornimmt, genauer: dabei „die Herrschaft über den letzten, unwiderruflich zum Tode führenden Akt innehat"82. \ 216 StGB wertet selbst das „ausdrückliche und ernstliche Verlangen des Getöteten zur Tötung“ lediglich als strafmildernden, nicht aber als unrechtsbeseitigenden Umstand („objektive Einwilligungsschranke") und bringt damit zum Ausdruck, dass verantwortungsbegründende „Autonomie“ in einer konsequent personalen Unrechtslehre nicht nur eine (auf gemäßigt indeterministischer Basis verstandene) ${ }^{83}$ „,freie Urheberschaft“ voraussetzt, sondern auch die „Rückführbarkeit des Geschehens auf eine Person als Subjekt des Geschehens" 84 , d.h. sog. „Steuerungsherrschaft" ${ }^{\text {"85 }}$ über den Tathergang. Beispielhaft veranschaulicht: „Straflos ist, wer das Gift mischt oder den

77 Siehe o. bei Fn. 53 ff.

78 In diesem Sinne Bottke, Suizid und Strafrecht, 1982, S. 248 ff.; Roxin, in: Wolter (Hrsg.), 140 Jahre Goltdammer`s Archiv für Strafrecht, 1993, S. 178 f.; ders., in: Roxin/Schroth (Hrsg.), Medizinstrafrecht, 2. Aufl. 2001, S. 104: aus Gründen der Rechtssicherheit; Münchener Kommentar zum Strafgesetzbuch (MK)/Schneider (Hrsg.: Joecks), 2003, Vor \$S 211 ff. Rn 54 ff.

79 Vgl. Geilen, JZ 1974, 145 ff.; Otto, in: Zaczyk (Hrsg.), Wolff-FS 1998, S. 395, 401; Schönke/Schröder/Eser (Hrsg.), Strafgesetzbuch, 27. Aufl. 2006, Vorbem. IS 211 ff. Rn 36; Wessels/Hettinger (Fn. 36), Rn 48 f.

80 MK/Schneider (Fn. 78), Vor \$S 211 ff. Rn 32; Klassiker: „Sirius-Fall“, vgl. BGHSt 32, 38 ff.

81 Zur bisher ungeklärten Frage einer Übertragung des Rechtsgedankens aus $₫ 25$ I Alt. 2 (mittelbare Täterschaft) bzw. \25 II StGB (Mittäterschaft) auf das Opferverhalten vgl. Duttge, in: Schmitz (Hrsg.), Otto-FS 2006 (im Erscheinen).

82 Statt vieler: Roxin, in: Medizinstrafrecht (Fn. 78), S. 104

83 Zur aktuellen Debatte um die normative Relevanz der neurophysiologischen Befunde vgl. aus rechtlicher Perspektive die Kritik bei Hillenkamp, JZ 2005, 313 ff.; Schreiber, in: Honnefelder/Sturma (Hrsg.), Jahrbuch für Wissenschaft und Ethik, Bd. 10 (2005), S. 23 ff.

84 Otto, Grundkurs Strafrecht. Allgemeine Strafrechtslehre, 7. Aufl. 2004, \6 Rn 44

85 Otto, ZStW 87 (1975), $539 \mathrm{ff}$. 
Revolver lädt, mit dem das „Opfer“"86 sich selbst tötet; dagegen ist nach \216 StGB strafbar, wer einen schwerkranken und sterbewilligen Menschen auf dessen Verlangen hin durch eine Injektion oder durch einen Revolverschuss tötet" ${ }^{\text {"87. }}$.

Weil das in \216 StGB verankerte Verbot der sog. aktiv-direkten Sterbehilfe de lege lata für sämtliche Lebenslagen, d.h. auch bei ,aussichtsloser Prognose“"88 strikte Geltung beansprucht, steht es unter dem Eindruck vor allem der niederländischen und belgischen Entwicklungen schon seit längerem im Blickpunkt der Aufmerksamkeit. Zwar ist die Vorschrift von den aktuellen Reformbestrebungen nicht erfasst und wird von den derzeit Verantwortlichen nimmermüde für sakrosankt erklärt ${ }^{89}$; doch begegnen in der (sowohl verfassungs- wie strafrechts-)wissenschaftlichen Debatte neuerdings vermehrt Stellungnahmen, die unter Rückgriff auf entsprechende Empfehlungen bereits in den 80er Jahren des letzten Jahrhunderts ${ }^{90}$ eine begrenzte Freigabe fordern ${ }^{91}$ oder gar meinen, eine Befugnis zur Tötung auf Verlangen für „seltene Ausnahmefälle“ unter Verweis auf den rechtfertigenden Notstand ( $\int 34$ StGB) bereits aus dem geltenden Recht deduzieren zu können ${ }^{92}$. Ingelfinger ${ }^{33}$ hat jedoch überzeugend auf die Problematik von „Notstands-Lösungen“ aufmerksam gemacht, die untrennbar mit einer externen Negativbewertung menschlichen Lebens und Zuschreibung als „lebensunwert" $" 94$ verbunden sind. Von solchem Verdikt möchte sich Lüderssen ausweislich seines kürzlich vorgetragenen Plädoyers „für die Legalisierung der aktiven Sterbehilfe“ fernhalten und betont deshalb nachdrücklich, dass ,,auslösendes Moment“ für dahingehende Überlegungen weder „bevölkerungspolitische“ Aspekte noch die Idee einer „Abwertung durch übermenschliche Lebenskonzepte“, sondern

86 Freiverantwortlich, d.h. mit hinreichender Einsichtsfähigkeit bzw. „Ernstlichkeit“!

87 Roxin, in: Medizinstrafrecht (Fn. 78), S. 104

88 So ausdrücklich BGHSt 37, 376 (Leitsatz 2): „Auch bei einer aussichtslosen Prognose darf Sterbehilfe nicht durch gezieltes Töten, sondern nur ... durch die Nichteinleitung oder den Abbruch lebenserhaltender Maßnahmen geleistet werden...".

89 Z.B. Zypries in ihrer Rede anlässlich der Vorstellung des Abschlussberichts der von ihr eingesetzten Arbeitsgruppe „Patientenautonomie am Lebensende“ (www.bmj.de vom 10.10. 2004); gegen die political correctness verstoßend dagegen Kusch, NJW 2006, 261 ff.

90 In diesem Sinne vor allem der Regelungsvorschlag des „Alternativ-Entwurfs Sterbehilfe“ 1986 (neu abgedr. in: Schöch/Verrel, GA 2005, 553, 557), den freilich der „AE-StB 2005“ aufgegeben hat (ebd., 582 ff.), sowie der 56. DJT (vgl. NJW 1986, 3065, 3073 f.)

91 Vgl. Lindner, JZ 2006, 373 ff.; Lüderssen, JZ 2006, 689 ff.; zuvor bereits Antoine, Aktive Sterbehilfe in der Grundrechtsordnung, 2004, S. 367 ff., 409; Wolfslast, in: Amelung (Hrsg.), Schreiber-FS, 2003, S. $913,922 \mathrm{ff}$.

92 So insbesondere R. Merkel, zuletzt in: Hoyer (Hrsg.), Schroeder-FS, 2006, S. 297, 320 f.; zuvor bereits Chatrilestas, Die Disponibilität des Rechtsgutes Leben in ihrer Bedeutung für die Probleme von Suizid und Euthanasie, 2001, S. 326 f.; Otto, Gutachten D zum 56. DJT, 1986, S. 60; Schmaltr, Rechtsvergleich Deutschland - USA, 2001, S. 66 f.

93 S. $246 \mathrm{ff}$.

94 Dazu unmissverständlich Arthur Kaufmann, Strafrecht zwischen Gestern und Morgen, 1983, S. 146: ,... ist ein Schritt in den Abgrund“. - Eingehende rechtshistorische Analyse zur einflussreichen Schrift von Binding/Hoche („Die Freigabe der Vernichtung lebensunwerten Lebens“, 1920) bei Scbumann, in: Riha (Hrsg.), Die Freigabe der Vernichtung lebensunwerten Lebens, 2005, S. 35 ff. 
ausschließlich „der Respekt vor dem Willen des Lebensmüden“ sei: Dort, wo „zum Beispiel Atemnot mit Erstickungsängsten, aktuelles Delirium mit epileptischen Anfällen, Irreversibilität des Heilungsprozesses im Sinne eines unaufhaltsamen Sterbeprozesses“ anzutreffen sei“, bestehe „die Vermutung (!), dass es im subjektiven Interesse (!) des Patienten liegt, sein Leben zu beenden“; das Prius sei daher stets seine „Autonomie“"95. Von der signifikanten Aufweichung der strikten Orientierung am Patientenwillen durch Übergang zu einer auf vermutete „Interessen“ gestützten Betrachtung einmal abgesehen, ist der Gedanke der Selbstbestimmung bekanntlich auch für die Entwicklung in den Niederlanden fortwährend in den Mittelpunkt der Erwägungen gerückt worden ${ }^{96}$, während selbige in der Sache schon seit langem eher von einer pseudo-objektiven „Güterabwägung“ (Lebenserhaltung versus ,unerträgliche Leiden“) geprägt gewesen ist ${ }^{97}$. Für die von $\int 216$ StGB erfasste Konstellation der ,freiwilligen Euthanasie“ bleibt aber begründungsbedürftig, warum selbst ein noch so vernünftig erscheinender Sterbewunsch nicht gleichermaßen wie bei der passiven Sterbehilfe ${ }^{98}$ den Respekt der Rechtsordnung vor dem Selbstbestimmungsrecht des Patienten aktiviert ${ }^{99}$.

Der Bonner Verfassungsrechtler Hillgruber hat kürzlich die Schutzpflichtdimension der Menschenwürdegarantie (Art. 1 I S. 2 GG) fruchtbar zu machen versucht mit dem Argument, dass im Falle des $\ 216$ StGB der Lebensmüde durch sein Verlangen den mangelnden „Wert“ seines Lebens manifestiere, was der Ausführende und damit Letztverantwortliche „übernehme“, so dass darin im Ergebnis eine mit Art. 1 I GG kollidierende „externe Bewertung eines menschlichen Lebens als lebensunwert“ liege ${ }^{100}$. Diese Bewertung ist aber zugleich von der Sichtweise des Rechtsgutsinhabers getragen, die sich nur dann als irrelevant ausweisen lässt, wenn diesem die Disponibilität entzogen ist. In der Sache verbirgt sich hinter dem Rückgriff auf die Menschenwürdegarantie daher aufs Neue eine Mystifizierung des Lebens im Sinne eines dem Menschen von vornherein entzogenen Gutes, wie es die Formel von der „Heiligkeit“ des Lebens ${ }^{101}$ zum Ausdruck bringt. Mögen die Verfassungsmütter und -väter die aktive Sterbehilfe auch für ganz und gar indiskutabel gehalten haben ${ }^{102}$, ist diese Auffassung jedoch in Art. 2 II GG als

\footnotetext{
95 Lüderssen, JZ 2006, 689 und 692

96 Vgl. etwa Admiraal, in: Kodalle (Hrsg.), Euthanasie - Das niederländische Modell, 2003, S. 40; Wils, Sterben. Zur Ethik der Euthanasie, 1999, S. 147 ff.

97 So explizit Lunshof, in: Thiele (Hrsg.), Aktive und Passive Sterbehilfe. Medizinische, rechtswissenschaftliche und philosophische Aspekte, 2005, S. 112 f.: „Der Gesetzgeber hat sich ... eindeutig für das ,Notlage-Modell' entschieden“; weiterhin Schreiber, in: Korras/Courakis (Hrsg.), AndroulakisFS, 2003, S. 637, 645: „notstandsähnliche Erwägungen“. Zur Entwicklungsgeschichte vgl. Tak, ZStW 113 (2001), 905, 907

$98 \quad \mathrm{Zu}$ dieser u. 4

99 So der Ansatz von Jakobs, Tötung auf Verlangen, Euthanasie u. Strafrechtssystem, 1998, S. 29 ff.

100 Hillgruber, ZfL 2006, Heft 3 (im Erscheinen)

101 Dazu o. bei Fn. 36

102 Dieses entstehungsgeschichtliche Argument stark betonend Hillgruber (Fn. 100).
} 
Rechtliche Typenbildung

sedes materiae zum Umgang mit menschlichem Leben nicht zum Ausdruck gekommen.

So bleiben für die Aufrechterhaltung des strikten Verbots im Wesentlichen nur noch zwei Anknüpfungspunkte, die bereits an anderer Stelle näher beschrieben worden sind ${ }^{103}$ : Der eine gedankliche Strang betont die Gefahren für den Erhalt des Tötungstabus und damit des zentralen Bestandteils einer jeden durch Generalprävention friedenstiftend und -erhaltend wirkenden Rechtsordnung („ordnungsschützende Funktion"). Missbrauchs- und Dammbruchszenarien lassen sich jedoch bei einem genaueren Blick auf die aktuelle Entwicklung in den Niederlanden nicht mehr als bloße Spekulation ${ }^{104}$ abtun: So weisen die im Auftrag der niederländischen Regierung seit 1991 (auf der Basis der alten Gesetzeslage) ${ }^{105}$ erhobenen Daten aus ${ }^{106}$, dass - vorbehaltlich des Dunkelfeldes - allenfalls in jedem zweiten der einschlägigen Fälle die gesetzlich vorgesehene und zur Herstellung von Transparenz schlechterdings unverzichtbare Meldung an die zur Überwachung zuständigen regionalen Kontrollkommissionen erfolgt ist; innerhalb dieses Anteils wurde zudem im Umfang von $82 \%$ gesetzeswidrig kein ärztlicher Kollege konsultiert. Seit 1991 weitgehend stabil ist außerdem die Zahl von jährlich etwa 900 bis 1.000 Fällen einer sog. „,nicht-freiwilligen Euthanasie“, die es nach dem Gesetz - es verlangt ein ausdrückliches Sterbeverlangen „nach reiflicher Überlegung“ - gar nicht geben dürfte. Im Untersuchungszeitraum von 1998 bis 2001 wurden von den Kontrollkommissionen etwa 7.000 Fälle untersucht, aber nur ganze acht (!) beanstandet, was den Verdacht weckt, die Vorschaltung eines justizfernen Gremiums bezwecke eher eine Immunisierung und Abkoppelung solcher Vorgänge von der Ermittlungstätigkeit der Strafverfolgungsbehörden. Inzwischen sind aus den letzten Jahren mindestens 22 Fälle der Tötung neugeborener Kinder bekannt geworden; für Alzheimer- und Demenzkranke gibt es bereits Überlegungen in Richtung einer expliziten Ausweitung des bisherigen Anwendungsbereiches. Aus diesen empirischen Befunden und Beobachtungen hat der Münchner Mediziner Oduncu erst unlängst das resignierende Fazit gezogen, dass die an sich eng begrenzte Tötungsbefugnis offensichtlich in keineswegs nur geringem Ausmaß missbraucht wird und dieser Missbrauch „durch die niederländische de factoLegalisierung [nicht nur] nicht verhindert“, sondern „durch die Duldung ... der Tötungen" erst maßgeblich „gefördert" worden sein könnte ${ }^{107}$. In diesem Lichte ${ }^{108}$ lassen sich weitere „Feldversuche“ um der auf dem Spiele stehenden Men-

\footnotetext{
103 Vgl. Duttge, ZfL 2004, 30, 35 f.

104 Polemisch MK/Schneider (Fn. 78), s 216 Rn 3 Fn. 12: „dumpfe Horror-Szenarien“.

105 Näher zur holländischen Entwicklung: Sagel-Grande, ZStW 111 (1999), 742 ff.

106 Zum Folgenden vgl. die Nachweise in: Duttge u.a. (Fn. 58), S. 20 ff., 54 ff.

107 Vgl. Oduncu, MedR 2005, 437, 443 f.

108 Der jüngste Bericht des „British Medical Journal“ (2006, 1110 ff.) weist überdies ein Anwachsen der Gesamtzahl aktiver Tötungen im dritten Jahr nacheinander aus.
} 
schenleben willen nicht verantworten ${ }^{109}$; wer dabei auf das Bestehen einer illegalen Praxis auch in Deutschland verweist ${ }^{110}$, unterliegt einem naturalistischen Fehlschluss.

Das zweite Argument geht von der Erkenntnis aus, dass schon bei der Selbsttötung das Vorliegen hinreichender Ernstlichkeit und damit die Befähigung zur tödlichen „Selbstbestimmung“ angesichts der Irreversibilität des Entschlusses zumeist mit erheblichen Zweifeln behaftet ist. Umso mehr muss dies aber dann gelten, wenn die Tat nicht selbst verübt, sondern auf eine andere Person delegiert werden soll. Hier stellt sich die grundsätzliche Frage, ob das Abwälzen der letztgültigen Entscheidung über die Herbeiführung des Todes auf einen anderen (qua „Beherrschung des todbringenden Augenblicks“ durch den Tötenden) ${ }^{111}$ nicht im Falle bestehender Handlungsfähigkeit des Sterbewilligen mehr als nur theoretische Zweifel an der Endgültigkeit und „Ernstlichkeit“ des Todesverlangens weckt. Roxin hat in diesem Zusammenhang beachtenswert zu bedenken gegeben: „Viele haben sich die Pistole schon an die Schläfe gesetzt, aber nur wenige hatten den Mut, abzudrücken“112. Für den Regelfall kommt dem Tötungsverbot des $\int 216$ StGB daher die Funktion einer Vorsorge gegenüber nur scheinbar „selbstbestimmten“ Todesverlangen zu („Voreiligkeitsschutz") ${ }^{113}$. Problematisch bleiben allerdings jene Sondergestaltungen, in denen wie im Fall Diane Pretty ${ }^{114}$ aufgrund gänzlicher Handlungsunfähigkeit und evidenter Aussichtslosigkeit der Lage jedenfalls ein sich fortlaufend wiederholender Sterbewunsch immer weniger die Annahme fehlender Freiverantwortlichkeit erlaubt. Hier dem unerträglich Leidenden unter Hinweis auf das allgemeine Tötungsverbot jede Hoffnung auf ein rascheres Ende nehmen zu müssen, erschiene in der Tat als ein Akt haarsträubender Unbarmherzigkeit. Entgegen landläufiger Auffassung bedarf es aber nicht etwa nur aus „moralischen Gründen“115 (welche „Moral“?), sondern von Rechts wegen eines Auswegs aus dem Dilemma. Die Lösung muss jedoch zugleich eine solche sein, mit der die Normgeltung und generalpräventive Kraft des strafrechtlichen Verbots möglichst ungeschmälert erhalten bleibt: Deshalb kann kein generell anwendbarer Rechtfertigungsgrund oder ein Tatbestandsausschluss nach dem „Vorbild“ des $\$ 218a I StGB, aber auch kein allgemeiner Entschuldigungsgrund etabliert werden. Diskussionswürdig ist vielmehr allein jener Weg, den der Bundesgerichtshof in Strafsachen bereits für den Fall eines der verbotenen Einfuhr und Überlassung von Betäubungsmitteln beschuldigten Sterbehelfers beschritten hat ${ }^{116}$ : Dort ge-

109 Auch Schreiber, in: Anhörung vor dem Ausschuß für Rechts- und Verfassungsfragen des Niedersächsischen Landtages, 69. Sitzung v. 18.1.2006, S. 14

110 So zuletzt Lüderssen, JZ 2006, 689, 693

111 Siehe o. Fn. 82

112 Roxin, in: 140 Jahre GA (Fn. 78), S. 177, 184

113 Dazu näher Duttge, ZfL 2004, 30, 34; wie auch MK/Schneider (Fn. 78), \216 Rn 8

114 EGMR NJW 2002, 2851 ff.; s.a. den Zivildienstleistenden-Fall, BGH NStZ 2003, 537 ff.

115 Simon, in: Düwell/Steigleder, Bioethik, 2003, S. 311

116 Vgl. BGHSt 46, 279, 291 
Rechtliche Typenbildung

langte er zu einer Verurteilung mit Strafvorbehalt ( $\$ 59$ StGB), die allerdings im Kontext des $\ 216$ StGB erst bei Einfügung eines (unbenannten) minderschweren Falles (und Anwendung von $\ 47$ Abs. 2 S. 2 StGB) zum Ziele führen kann ${ }^{117}$.

3. Dogmatisch-strukturell zählt zur „aktiven“ Sterbehilfe auch die (gezielte) Gabe schmerzstillender Medikamente, sofern damit ein beschleunigter Todeseintritt einhergehen könnte, der um der Leidensminderung willen ggf. als unvermeidbare Nebenfolge in Kauf genommen wird. Denn in solchem Falle resultierte der lebensverkürzende Effekt auch hier nicht etwa aus der „desintegrierenden“ Wirkung der Erkrankung ${ }^{118}$, sondern aus einer Intervention „,von außen“119, wenngleich im Regelfall nur als nicht auszuschließende Eventualität, kaum je als forensisch beweisbares Faktum ${ }^{120}$. In jüngerer Vergangenheit wird allerdings aufgrund neuerer empirischer Studien bezweifelt, ob sich mit einer korrekt angewandten Symptomlinderung mittels der gebräuchlichen Opiate und Sedativa überhaupt ein derartiger Effekt verbindet; eine Züricher Gruppe von Rechtsmedizinern gelangte erst kürzlich zu dem Resultat, dass „eine lebensverlängernde Wirkung, u.a. wegen der durch die Symptomlinderung erzielten Stressminderung, zumindest ebenso plausibel" sei121. Offenbar besteht hierüber aber bisher keine hinreichende Klarheit, so dass jedenfalls für seltene Fälle auch die Möglichkeit nachteiliger Wirkungen auf den menschlichen Organismus einkalkuliert werden muss ${ }^{122}$. Hiervon gehen im Übrigen ebenfalls die „Grundsätze“ der Bundesärztekammer aus und bestimmen: „Bei Sterbenden kann die Linderung des Leidens so im Vordergrund stehen, dass eine möglicherweise unvermeidbare Lebensverkürzung hingenommen werden darf" ${ }^{\prime \prime 23}$.

$\mathrm{Da}$ ärztliches Handeln nicht auf die Lebenserhaltung beschränkt ist, sondern ebenso die Linderung von Leiden bezweckt ${ }^{124}$, besteht heute auch im juristischen Kontext „nahezu einhelliger Grundkonsens“125 über die Zulässigkeit (und bei Ineffizienz weniger eingriffsintensiver Mittel sogar Gebotenheit) solcher hinsichtlich der Todesfolge nur mit voluntarium indirectum verübten, weil im Gegensatz zur Konstellation des $₫ 216$ StGB in der Hauptsache auf die Leidminderung gerichteten „Sterbehilfe“. Der Bundesgerichtshof in Strafsachen hat fallbedingt den Rahmen des Erlaubten zunächst allerdings (und die Bundesärztekammer ihm

\footnotetext{
117 Näher Duttge, JZ 2006 [im Erscheinen]; s.a. Otto, Gutachten D zum 56. DJT, 1986, S. 93

118 Treffend Höfling, JuS 2000, 111, 113: ,innerorganismischer Desintegrationsprozeß“.

119 Wie hier z.B. auch Schreiber, NStZ 2006, 473, 475

120 Zutreffender Hinweis bei Geilen, Euthanasie und Selbstbestimmung 1975, S. 23

121 Vgl. Bosshard/Faisst (Hrsg.) u.a., Ethik in der Medizin 2006, 120, $123 \mathrm{f}$.

122 Weiterführende Literatur zit. bei Duttge u.a. (Fn. 58), S. 81

123 Bundesärztekammer (o. Fn. 67), Ziff. I, Absatz 3, Satz 2

124 Vgl. § 1 II der Musterberufsordnung für die deutschen Ärztinnen und Ärzte, www.bundesaerztekammer.de/30/Berufsordnung/index.html (Stand: 03.09.2006)

125 BGHSt 46, 279, 284
} 
folgend) auf „Sterbende“ begrenzt ${ }^{126}$, was inzwischen allgemein als zu restriktiv angesehen wird. Die Notwendigkeit einer gesetzlichen Rahmenregelung zeigt sich daher in den jüngeren Stellungnahmen und Empfehlungen (Arbeitsgruppe „Patientenautonomie am Lebensende ${ }^{\text {"127 }}$, Alternativ-Entwurf Sterbebegleitung ${ }^{128}$, Gutachten zum 66. Deutschen Juristentag ${ }^{129}$ ) mit Recht bereits in dem Anliegen, diese sachlich nicht zu rechtfertigende Beschränkung auf die sog. „Terminalphase“ aufzugeben. Zugleich bedarf es jedoch auch einer Grenzziehung „,in subjektiver Hinsicht" ${ }^{\text {"130, }}$ seit die bisherige Verknüpfung mit dem „bedingten Vorsatz“ (d.h. lediglich Inkaufnahme der unvermeidlichen Nebenfolge) nicht mehr selbstverständlich ist und insbesondere Alternativ-Entwurf (AE-StB) und Juristentagsgutachten für eine Einbeziehung auch des sicheren Wissens (sog. dolus directus 2. Grades) ${ }^{131}$ eintreten. Auf diese Frage vermag eine gesetzliche Regelung aber nur dann eine sachlich überzeugende Antwort zu geben, wenn Klarheit herrscht über den Rechtsgrund der Straffreistellung in Abgrenzung zu \216 StGB. Es greift daher wesentlich zu kurz, qua gesetzgeberischem „Basta“ ein Ende der (vermeintlich) „unnötigen strafrechtswissenschaftlichen Diskussion mit ihren unterschiedlichen Theorien ..." anzumahnen ${ }^{132}$. Das Gegenteil ist richtig: Erst eine zutreffende Aufklärung des vorfindlichen Wertekonflikts und insbesondere der Frage einer Betroffenheit vorwiegend des Selbstbestimmungsrechts oder anstelle dessen der ärztlichen Fürsorgepflicht (,medizinische Indikation“) ${ }^{133}$ weist den Weg zu einer sachgerechten gesetzlichen Regelung.

Ist doch der strafrechtsdogmatische Theorienstreit keineswegs nur harmloses Glasperlenspiel: Die wohl h.M. will bekanntlich die lebensverkürzende Leidminderung primär auf der Basis des rechtfertigenden Notstandes ( $\int 34$ StGB) anerkennen, weil - so der Bundesgerichtshof - „die Ermöglichung eines Todes in Würde und Schmerzfreiheit gemäß dem erklärten oder mutmaßlichen Patientenwillen $^{134}$... ein höherwertiges Rechtsgut ... [sei] als die Aussicht, unter schwersten, insbesondere sog. Vernichtungsschmerzen noch kurze Zeit länger leben zu müssen“135. Hier ist nun leicht zu erkennen, dass mit dieser suggestiv auf die Men-

\footnotetext{
126 BGHSt 42, 301, 305; BGHSt 46, 279, 285 spricht allerdings vom „tödlich Kranken“.

127 In Auszügen abgedruckt bei: Duttge u.a. (Fn. 58), S. 103 ff.

128 Schöch/Verrel, GA 2005, 553, 555

129 Verrel (Fn.15), C 103: „Das humane Anliegen der Palliativmedizin erfasst alle Fälle des Erleidens krankheitsbedingter Qualen“.

130 Verrel (Fn.15), C 102

131 Zur klassischen Dreiteilung des Vorsatzes vgl. etwa Roxin, Strafrecht Allgemeiner Teil, Bd. I,

4. Aufl. 2006, \12 Rn $1 \mathrm{ff}$.

132 So aber Schumann, Dignitas - Voluntas - Vita, 2006, S. $81 \mathrm{f}$.

133 In letzterem Sinne Schumann (ebd.), S. 34, 82

134 Insoweit besteht eine Vermischung mit dem Rechtfertigungsgrund der (mutmaßlichen) Einwilligung, dazu sogleich im Text.

135 BGHSt 42, 301, 305; explizit zust. Rosenau, in: Heun/Honecker u.a. (Hrsg.), Evangelisches Staatslexikon, 2006, Sp. 2370; dezidiert auf $\$ 34$ StGB abstellend: Otto, NJW 2006, 2217, 2221
} 
schenwürdegarantie rekurrierenden Formel wiederum eine Dimension von „objektiver" Bewertung menschlichen Lebens nach zugeschriebener "Qualität" (Schmerzfreiheit/Vernichtungsschmerzen) Eingang findet, die auch deshalb in höchstem Maße gefährlich ist, weil sich von hier aus nicht nur (mit dem AE-StB und dem Gutachten zum DJT) die sichere, sondern sogar die gezielte Tötung (sog. dolus directus 1. Grades) ${ }^{136}$ rechtfertigen lässt. Mehr noch: Es wäre in diesem Lichte gar nicht mehr einzusehen, warum überhaupt jemals noch mit der „Giftspritze“ gezögert werden sollte, wenn es darum geht, einen „,unerträglich Leidenden“ endlich von seinen Qualen zu erlösen. Mit anderen Worten würde mit einer Notstandsrechtfertigung der indirekten Sterbehilfe gleichsam ein „Trojanisches Pferd“ geschaffen, mit dem sich die eiserne Türe zur verbotenen Tötung auf Verlangen leicht öffnen ließe. Diese Argumentation ist mitnichten eine nur theoretisch mögliche, sondern ganz im Gegenteil inzwischen auf interessierter Seite schon längst in stetigem Gebrauch ${ }^{137}$.

Dass aus „objektiver“ Warte die Schmerzlinderung den - sonst als „höchstrangig“ bezeichneten - Belang der Lebenserhaltung wesentlich überwiege (vgl. \ 34 S. 1 StGB), bleibt jedoch so lange bloße Behauptung, wie es an einem (abschlieBenden) Katalog von Abwägungskriterien und einem (wenigstens ansatzweise) transparenten Abwägungsmaßstab fehlt. Üblicherweise wird deshalb - mit der Rechtsprechung des Bundesgerichtshofs ${ }^{138}$ - eine solche Abwägung an den „erklärten oder mutmaßlichen Patientenwillen“" gebunden, was aber unvermeidlich die Frage aufwirft, warum dann die Legitimation nicht sogleich in der (mutmaßlichen) Einwilligung gesucht wird ${ }^{139}$. Ausgehend von der Prämisse einer Rechtfertigungsbedürftigkeit jedweden ärztlichen Eingriffs darf die Patientenautonomie auch bei der Schmerzmedikation nicht ausgehöhlt werden; denn welche Leiden zu welchem „Preis“ nicht mehr erduldet werden sollen, kann nur der jeweils Betroffene selbst beurteilen. Den Bezug zur Menschenwürdegarantie richtig gestellt geht es hier also mit dem Freiburger Strafrechtler Frisch um den ,uneinschränkbaren, weil in der Menschenwürde wurzelnden Kernbereich des Selbstbestimmungsrechts" "140. Oder mit dem amerikanischen Rechtsphilosophen Ronald Dworkin: Den Patienten zu nötigen, auf eine Weise zu leben (oder zu sterben), die nach Meinung anderer die „richtige“ ist, bildet einen Akt „menschenverachtender Tyrannei“" ${ }^{\text {“141. }}$

Gegen eine solche „Einwilligungslösung“ wird freilich geltend gemacht, dass sie im Hinblick auf $\int 216$ StGB keine ausreichende Legitimation bewirken könne.

\footnotetext{
$136=$ „Absicht“, vgl. Roxin (Fn. 131), $\$ 12$ Rn 7 ff.

137 So v.a. R. Merkel, zuletzt in: Hoyer (Hrsg.), Schroeder-FS, 2006, S. 297, 321

138 Siehe o. bei Fn. 134; statt vieler etwa MK/Schneider (Fn. 78), Vor \$S 211 ff. Rn 103

139 Wie hier auch Roxin, in: Medizinstrafrecht (Fn. 78), S. 90 f.; Verrel, JZ 1996, 224, 226 f.

140 Frisch, in: Leipold (Hrsg.) Selbstbestimmung in der modernen Gesellschaft aus deutscher und japanischer Sicht, 1997, S. 108; siehe auch zu Art. 1 GG treffend MK/Schneider (Fn. 78), Vor SS 211 ff. Rn 102

141 Dworkin, Die Grenze des Lebens, Abtreibung, Euthanasie und persönliche Freiheit, 1994, S. 208 ff.
} 
Im Vordergrund stehe der Therapiecharakter, der ,zu einem gewissen Autonomieverlust beim Moribunden“ führe ${ }^{142}$. Weil jedoch auch „die alleinige Anwendung des $\int 34$ StGB wegen der problematischen Rechtsgüterabwägung nicht völlig überzeug(e)“, spricht sich der AE-StB für eine Art „Kombination“ von Notstand und Einwilligung aus, wonach die Einwilligung als ,gewichtiger Faktor bei der Rechtsgüterabwägung zugunsten des selbstbestimmten Sterbens in Würde" begriffen werden müsse ${ }^{143}$. Dies wird man jedoch schon aus Gründen der Logik kaum als plausiblen Lösungsansatz ansehen können, weil unerfindlich bleibt, wie zwei für sich (angeblich) nicht taugliche Rechtfertigungsgründe bei ihrer „Zusammenschau“ das Erlaubtsein begründen können sollen. Pointiert: Wenn es an den Voraussetzungen eines Erlaubnissatzes fehlt, kann sich an seiner Nichtanwendbarkeit nicht dadurch etwas ändern, dass auch die Anforderungen eines weiteren Rechtfertigungsgrundes nicht erfüllt sind. In Wahrheit ist eine solche „Rosinentheorie“ vor allem geeignet, die gesetzlichen Anforderungen zu unterlaufen, was insbesondere mit Blick auf den schon erwähnten Erweiterungsvorschlag des AE-StB um den Bereich der sicheren Lebensverkürzung erhellt ${ }^{144}$ : Einer solchen „Tötung durch leidensmindernde Behandlung“, die sich von einer „Leidensminderung durch Tötung“ kaum noch unterscheiden ließe ${ }^{145}$, steht die Einwilligungsschranke des $\int$ 216 StGB entgegen, abgesehen davon, dass die Gabe eines Schmerzmittels mit absehbar tödlicher Wirkung wohl kaum noch mit den Regeln der ärztlichen Kunst verträglich sein dürfte; der Vorschlag des AE-StB läuft daher letztlich doch - gegenteiliger Beteuerung zum Trotz - auf eine diffuse Notstandsrechtfertigung hinaus.

Anders liegt es hingegen, soweit entsprechend dem bisherigen Anwendungsbereich die lebensverkürzende Wirkung nur mehr nicht ausgeschlossen werden kann und um des (hier nun richtig verankerten) therapeutischen Zweckes willen ${ }^{146}$ lediglich als mögliche Folge notgedrungen in Kauf genommen werden soll: Hierdurch wird niemand „zur Tötung bestimmt“ ( $\int 216$ StGB), so dass die Einwilligungssperre insoweit gar nicht greift ${ }^{147}$. Mit anderen Worten: Es ist die begrenzte Wirkkraft der rechtfertigenden (bzw. mutmaßlichen) ${ }^{148}$ Einwilligung, resultierend aus der (sachlich überzeugenden) Wertentscheidung des Gesetzgebers, die dogmatisch die Trennlinie zwischen strafbarer direkter und erlaubter indirekter Sterbehil-

\footnotetext{
142 So bereits Geilen (Fn. 120), S. 23

143 Schöch/Verrel, GA 2005, 553, 574; w. Nachw. bei MK/Schneider (Fn. 78), Vor SS 211 ff. Rn 99

144 Vgl. Schöch/Verrel, GA 2005, 553, 575 f.; ebenso Verrel (Fn. 15), C 102 f.

145 Schreiber, NStZ 2006, 473, 475, spricht zu Recht von einem nur ,sehr schmalen Grat der Unterscheidung“".

146 Die therapeutische Zwecksetzung kommt somit als weiterer Aspekt hinzu, kann jedoch bei Vermeidung von Zwangsbehandlungen die Legitimation allein nicht bewirken.

147 Was bisher allgemein übersehen wird, ausf. Duttge u.a. (Fn. 58), S. 82 ff.

148 Dass die Linderung quälender Schmerzen bei (bloß) nicht ausschließbarer Lebensverkürzung mutmaßlich von allen (noch schmerzempfindungsfähigen) Patienten gewollt sein wird, lässt sich anders als bei sicherer Todesherbeiführung - ohne weiteres annehmen.
} 
Rechtliche Typenbildung

fe erzwingt. Für letztere hingegen - so der AE-StB mit einer im Vordringen befindlichen strafrechtlichen Lehre - einerseits eine Rechtfertigung qua Einwilligung unter Verweis auf $\int 216$ StGB abzulehnen und ,wegen der Unantastbarkeit fremden Lebens“ am Verbot des \216 StGB festzuhalten, andererseits jedoch „im fliegenden Wechsel“ zum „flexibler" handhabbaren Notstand den erlaubten Bereich aktiver Lebensverkürzung (!) auf den direkten Vorsatz ausweiten zu wollen, ist wertungswidersprüchlich.

Die skizzierte Sichtweise führt dagegen die beiden gleichermaßen einschlägigen Belange des Selbstbestimmungsrechts sowie der ärztlichen Fürsorge zu einer harmonischen Einheit zusammen: Die Schmerzmedikation darf nicht ohne Beachtung des Patientenwillens erfolgen; selbiger legitimiert aber keine sichere Tötung, die zugleich dem Gebot des „Wohltuns“ widerspräche. Die vom AE-StB wie auch im Gutachten zum 66. Deutschen Juristentag vorgeschlagene Pflicht zur Dokumentation von Dosierung und Therapieverlauf wie auch zur Konsultation eines weiteren Arztes bei höherer Dosierung („Vieraugenprinzip“) ${ }^{149}$ bilden geeignete Instrumente, um einem Missbrauch in Form einer ,als Schmerzbehandlung getarnten aktiven Sterbehilfe" 150 vorzubeugen. Hiervon kann jedoch auch die sog. „terminale Sedierung“, d.h. eine äußerstenfalls eingesetzte medikamentöse Schmerzlinderung, die das Bewusstsein des Patienten weitgehend oder vollständig ausschließt, nicht einfach unter Verweis auf einen Zurechnungsausschluss qua „erlaubtem Risiko“ bzw. „einverständlicher Fremdgefährdung“'151 ausgenommen werden ${ }^{152}$. Soweit eine solche Sedierung - nicht ausschließbar - lebensverkürzend wirkt, bildet sie einen Sonderfall der - insoweit erlaubten - indirekten Sterbehilfe ${ }^{153}$.

4. Sämtliche der bisher erörterten Konstellationen zeigen das einem „Wertekonflikt"154 immanente gegenläufige Zusammentreffen zentraler medizinethischer Prinzipien, freilich mit divergierender Auflösung: Während im Falle der Tötung auf Verlangen nicht nur die ärztliche Fürsorgepflicht („Wohltun“), sondern vor allem auch das davon gesonderte ${ }^{155}$ Schädigungsverbot und das im juristischen Kontext fundamentale Anliegen einer Erhaltung des Rechtsfriedens und einer widerspruchsfreien Rechtsordnung ${ }^{156}$ in ihrer Wertigkeit insgesamt das Selbstbe-

\footnotetext{
149 Schöch/Verrel, GA 2005, 577, 586; Verrel (Fn. 15), C 109

150 Schöch/Verrel, GA 2005, 553, 578

151 Die nach h.M. - im Unterschied zur eigenverantwortlichen Selbstgefährdung - die Zurechnung gerade nicht ausschließt, dazu auch Duttge, in: Schmitz, Otto-FS 2006 [im Erscheinen].

152 So aber Schöch/Verrel, GA 2005, 553, 575; bzgl. des gesamten Anwendungsbereichs der indirek-

ten Sterbehilfe: Schumann (Fn. 132), S. 33 f.

153 Dazu m.w.N. Duttge u.a. (Fn. 58), S. 86 ff.

154 Vgl. Kreß, Medizinische Ethik, 2003, S. 162

155 Zum „Georgtown-Mantra“ vgl. Beauchamp/Childress, Principles of Biomedical Ethics, 2001:

Respekt gegenüber der patientenseitigen Autonomie, Schädigungsverbot, Gebot des Wohltuns

(„Fürsorge") und Prinzip der Gerechtigkeit.

156 Zutreffend Schumann (Fn. 132), S. 59; zweifelnd dagegen Kun₹, in: Donatsch (Hrsg.), Trechsel-FS 2002, S. 626 f.
} 
stimmungsrecht deutlich überragen ${ }^{157}$, das in seiner lebensweltlichen Substanz aufgrund der Delegation ${ }^{158}$ und hier regelmäßig gegebenen hohen Sicherheit ${ }^{159}$ des (irreversiblen) Tötungsgeschehens ohnehin in besonderem Maße mit Zweifeln behaftet ist, zieht eine Veränderung der Gewichte sowohl bei der indirekten Sterbehilfe ${ }^{160}$ als auch beim assistierten Suizid das gegenteilige Abwägungsergebnis nach sich. In letztgenanntem Fall gewinnt, soweit es nicht an der nötigen „Freiverantwortlichkeit“ fehlt ${ }^{161}$, das Selbstbestimmungsrecht ausschlaggebende Bedeutung, weil es in dessen Kernbereich fällt, wie sich der Einzelne zu sich selbst verhält. Die „Selbstherrschaft“, die sich mit dem Begriff der Selbstbestimmung jedenfalls primär verbindet, verheißt dem Einzelnen die Freiheit, von Außeneinwirkungen möglichst unbeeinflusst ,sein Schicksal selbst in die Hand zu nehmen“162. Dem Einzelnen auch dann, wenn Defizite in seiner Entscheidungsfindung oder Willensbildung nicht mehr feststellbar sind, allein wegen des Ergebnisses jener Entschließung den Respekt zu versagen, käme einem „harten Paternalismus“"163 gleich, der letztlich den Status des Entscheidungsträgers als Subjekt rundweg negiert. In einer freiheitlich verfassten Gesellschaft kann daher von den beiden Ausprägungen jener Haltung, die sich in gleicher Weise mit der „Achtung einer Person"164 verbinden, im Kollisionsfalle nur derjenigen der Vorrang zukommen, die den Respekt vor den Wünschen dieser Person zum Gegenstand hat. Hingegen die Sorge um deren „Woht $\boldsymbol{L}^{6}$ in den Mittelpunkt zu stellen, würde verkennen, dass externe und interne Sicht nicht übereinstimmen müssen (und es regelmäßig auch nicht tun). Mit dem britischen Medizinethiker John Harris: „Ganz gleich, was unserem Leben in unseren Augen seinen Wert verleiht, und ganz gleich, wie sehr sich das von dem unterscheidet, was dem Leben anderer in deren Augen Wert verleiht; unser Leben verliert für uns seinen Wert in dem Maße, in dem wir daran gehindert werden, den Dingen nachzugehen, denen wir nachgehen wollen“, oder kurz gefasst: „Die Autonomie ... [ist essentieller] Teil dessen, was es heißt, das Leben als wertvoll anzusehen" ${ }^{\prime 165}$.

\footnotetext{
157 Der vorstehende Vorschlag eines Schuldspruchs ohne Strafverhängung für seltene Ausnahmefälle (o. bei Fn. 116 f.) nimmt gerade Rücksicht auf die Geltung des Tötungsverbots.

158 Dazu o. bei Fn. 111

159 Zu Recht betont bei Beauchamp, in: Sass (Hrsg.), Medizin und Ethik, 1989, S. 271: „Während die aktive Auslöschung des Lebens der Patientin jede Lebenschance nimmt, bleibt ihr bei einem passiven Abbruch lebensverlängernder Maßnahmen diese Chance erhalten“".

160 Auf ihr bisheriges Anwendungsfeld (dolus eventualis) beschränkt ist das Schädigungsverbot nicht in seinem Kerngehalt betroffen und legen Fürsorgepflicht und Selbstbestimmungsrecht eine insoweit riskante Schmerzmedikation nahe.

161 Zur sonst in Betracht kommenden Fremdtötung in mittelbarer Täterschaft s.o. bei Fn. 80.

162 Harris, Der Wert des Lebens (dt. Ausgabe hrsg. von Ursula Wolf), 1995, S. 281 (Erstausgabe:

The Value of Life, 1985)

163 Dazu näher - und zu Recht ablehnend - R. Merkel, in: Hegselmann/Merkel (Hrsg.), Zur Debatte über Euthanasie, 1991, S. 82 f.

164 Harris (Fn. 162), S. 268

165 Harris (Fn. 162), S. 277
} 
Hierin liegt der tiefere Grund für die im juristischen Kontext inzwischen nahezu unbestrittene Auffassung, dass dem Bestimmungsrecht des Einzelnen über seine „leibliche Integrität“ als „ureigenster Bereich seiner Persönlichkeit" ${ }^{\text {"166 }}$ die Notwendigkeit einer jenseits der ärztlichen Vernunft liegenden Legitimation von Heileingriffen immanent ist. Die Idee einer konzeptionell als „Zwei-SäulenModell“167 darstellbaren „Verantwortungspartnerschaft“168 impliziert, dass dem Patienten jedenfalls in aktuellen Behandlungssituationen ein durchsetzungsfähiges (zivil- wie strafrechtlich ${ }^{169}$ abgesichertes) Vetorecht zukommt, mag eine medizinische Intervention aus ärztlicher Sicht auch noch so sehr (selbst vital) indiziert $\operatorname{sein}^{170}$. Demzufolge sperrt bereits der Ausfall auch nur einer „Säule“ die Zulässigkeit des Eingriffs, so dass eine Therapiebegrenzung sowohl wegen Nicht(-mehr-) Bestehens der medizinischen Indikation als auch bei Ausübung des patientenseitigen Vetorechts nicht nur erlaubt, sondern sogar geboten ist. Dies ist der Bereich der (erlaubten) sog. „passiven Sterbehilfe“, die das (gezielte) Nichteinleiten, die Einstellung oder Reduktion lebenserhaltender Maßnahmen zum Gegenstand hat.

Dabei sollte freilich nicht übersehen werden, dass die betonte Rechtfertigungsbedürftigkeit der (Weiter-)Behandlung eine grundlegende „ethische Differenz zwischen Töten und Sterbenlassen" ${ }^{171}$ impliziert, die sich erst vor dem Hintergrund einer Unterscheidung in Rechts- und Tugendpflichten ${ }^{172}$ (Gerechtigkeit und Menschenliebe) und eines damit - durchaus in Übereinstimmung mit der hippokratischen Tradition (,,primum nil nocere") - verbundenen Vorrangs des Eingriffsverbots erklärt ${ }^{173}$. Die sich von solcher „negativen Verantwortung“ abhebende „positive Verantwortung"“174 löst sich damit aber nicht gleichsam in Luft auf, weil auch im Falle des Nicht- bzw. Nicht-so-Handelns in ausschlaggebender Weise (und im Übrigen ebenso gezielt) auf den Lauf der Dinge Einfluss genom-

166 BVerfGE 52, 131, $171 \mathrm{ff}$.

167 Vgl. Laufs, NJW 2000, 1757, 1760 m.w.N.; siehe auch die Modellbildung bei E. Emanuel/L. Emanuel, Journal of the American Medical Association Bd. 267 (1992), 2221 ff.: paternalistisches, informatives, interpretatives und deliberatives Modell.

168 Treffend Bioethik-Kommission des Landes Rheinland-Pfalz (Fn. 65), S. 30; klassisch: Jaspers, Arzt und Patient, in: Der Arzt im technischen Zeitalter, 1986, S. 19; siehe weiterhin Laufs, in: Laufs/Uhlenbruck (Hrsg.), Handbuch des Arztrechts, 3. Aufl., 2002, \ 61 Rn 1 Fn. 1: ,therapeutisches Arbeitsbündnis“; aber auch Wieland, Strukturwandel der Medizin und ärztlichen Ethik, 1986, S. 58 ff. zum „Strukturwandel der Medizin“ in Richtung einer „Anonymisierung“ des Arzt-Patienten-Verhältnisses.

169 Ungeachtet der Bemühungen um Etablierung eines Sondertatbestands gegen die „eigenmächtige Heilbehandlung“, dazu etwa Schroeder, Besondere Strafvorschriften gegen Eigenmächtige und Fehlerhafte Heilbehandlung?, 1998

170 Grdl. BGHSt 11, $111 \mathrm{ff}$.

171 Verrel (Fn. 15), Gutachten C zum 66. DJT, S. 64

172 Vgl. dazu in kantischem Verständnis anlässlich der Suche nach dem „Absoluten“ im Recht:

Duttge/Löwe, in: Joerden/Byrd (Hrsg.) u.a., Jahrbuch für Recht und Ethik, Bd. 14, 2006 (im Erscheinen)

173 Dazu näher Höffe, Medizin ohne Ethik?, 2002, S. 45, der die Mißachtung dieses Vorrangverhältnisses als „humanitaristischen Fehlschluß“ bezeichnet.

174 Begriffe nach Harris (Fn. 162), S. 61 ff. 
men werden kann: „Denn wenn wir uns dagegen entscheiden, jemanden zu behandeln oder ihm zu Hilfe zu kommen, und dies in dem Wissen, dass diese Person in der Folge sterben oder leiden wird, ist ... ihr Tod oder Leiden in beiden Fällen eine Folge unserer Entscheidung“"175. Dem ärztlichen Gewissen ist dieser Zusammenhang nur allzu bewusst, und aus dem sich hieraus ergebenden Dilemma führt erst die schon eingeführte Erkenntnis, dass in einer auf dem Fundament individueller Freiheit erbauten Rechtsordnung keine überzeugende Rechtfertigung für Zwangsbehandlungen zu finden ist. Dies gilt aber nur für den Fall hinreichender „Mündigkeit", so dass Maßnahmen zur Beförderung einer ,größtmöglichen Autonomie" 176 nicht nur mit der Freiheitsprämisse ohne weiteres vereinbar, sondern aus der Perspektive des Rechts - als Ausdruck sozialstaatlich motivierter „Freiheitsvorsorge“ 177 geradezu denknotwendige Konsequenz derselben sind. Aus der (möglichen) Irreversibilität einer Entscheidung gegen den Einsatz lebenserhaltender Maßnahmen folgt angesichts der hier ganz erheblichen Unwägbarkeiten bei der Feststellung hinreichender „Mündigkeit“ die Maxime größtmöglicher „Risikominimierung “178: „Wo eine Entscheidung von so fundamentaler Bedeutung für eine Person ist“, handelt jeder gerechtfertigt, „der sein Bestes tut, um ... dem anderen dabei zu helfen, sie so autonom wie möglich zu gestalten“"179.

Drei Folgerungen lassen sich aus dieser Grundlegung auf der Prinzipienebene ziehen: Erstens befindet sich der Arzt dort, wo keine hinreichende Sicherheit über das Vorliegen einer „wohlüberlegten“ Entscheidung des Patienten (dessen höchstpersönlichem Wertehorizont entsprechend) besteht, infolge seiner spezifischen Hilfeleistungs- und Fürsorgepflicht nicht in einer Situation nur einseitiger Rechtfertigungsbedürftigkeit, sondern - von der vorherrschenden Sichtweise gerne übersehen ${ }^{180}$ - in einer solchen mit Entscheidungszwang zwischen zwei rechtfertigungsbedürttigen Handlungsalternativen bei asymmetrischer Rechtsgutsgefährdung (Selbstbestimmungs- versus Lebensrecht ${ }^{181}$ ). Dies gilt zweitens vor allem für die besonders problematische Konstellation eines im Entscheidungszeitpunkt einwilligungs- oder gar handlungsunfähigen Patienten. Die aktuelle Rechtspolitik schließt bekanntlich vom Vetorecht des Patienten in einwilligungsfähigem Zustand auf das

\footnotetext{
175 Harris (Fn. 162), S. 92; vgl. auch Birnbacher, Tun und Unterlassen, 1995, S. 350: Über Leben „verfügen heißt doch: die Dauer eines Lebens nach eigenen Vorstellungen zu bemessen ...; in diesem Sinne ist aber auch die passive Sterbehilfe ... ein ,Verfügen' über sein Leben“.

176 Vgl. Harris, ebd.: „Man ist ... in dem Maße autonom, in dem die Entscheidungen, die man trifft, authentisch sind, von anderen nicht behindert werden und so wenig wie möglich mit Mängeln behaftet sind“.

177 Duttge, Zeitschrift für Palliativmedizin, 2006, 48, 51

178 Zu den einzelnen Risiken näher Birnbacher (Fn. 175), S. 349 ff.

179 Harris (Fn. 162), S. 281

180 Z.B. Lipp, Patientenverfügung und Lebensschutz, 2005, S. 12

181 Denn im Falle nicht mit hinreichender Gewissheit gewollter Begrenzung einer medizinisch indizierten Behandlung verletzt der Therapieabbruch fundamental das Recht, weiterleben zu dürfen. Mit einer - fragwürdigen (s.o. bei Fn. 41) - Verabsolutierung des Lebensschutzes hat dies daher nichts zu tun!
} 
Bedürfnis nach einer „Verlängerung“ des Selbstbestimmungsrechts in das Stadium späterer Einwilligungsunfähigkeit mit überzeugungskräftigem Hinweis auf die auch insoweit Beachtung gebietende Rechtssubjektivität eines jeden Menschen ${ }^{182}$. Hier mit dem Instrument der Vorausverfügung („Patientenverfügung“) Abhilfe zu schaffen, um auf diese Weise dennoch an eine authentische Willensbekundung anknüpfen zu können, ist ein nahe liegender Gedanke, der insbesondere auch mangels besserer Alternativen ${ }^{183}$ Unterstützung verdient. Allerdings handelt es sich dabei entgegen einem des Öfteren begegnenden Anschein keineswegs um einen „Königsweg“, sondern eher um die Wahl des kleineren „Übels“. Denn der einer jeden Patientenverfügung immanente „Zeitsprung“ wirft nicht etwa nur hinsichtlich eines möglichen Missbrauchs, sondern vor allem auch hinsichtlich der Frage, ob es sich bei dem Erklärten überhaupt um einen „wohlüberlegten“ und noch immer aktuellen Ausdruck patientenseitiger „Autonomie“ handelt, eine Reihe von Zweifelsfragen auf. Sollen die hiermit einhergehenden Bedenken ${ }^{184}$ auch nur annäherungsweise entkräftet werden, bedarf es zwingend der Implementierung formeller und verfahrensrechtlicher Sicherungen, die bereits an anderer Stelle näher verhandelt worden sind ${ }^{185}$.

Der vorherrschende rechtspolitische Zeitgeist will hingegen auf solche Anforderungen - glücklicherweise vom Schriftformerfordernis meist abgesehen (an Terri Schiavo sei erinnert) ${ }^{186}$ - um der erstrebten „Liberalität“" willen verzichten, insbesondere auch auf eine Pflicht zur Aktualisierung des Verfügten und zur vorausgehenden (insbesondere medizinischen) Beratung. Wie sich freilich aus einer mehr oder minder weit in der Vergangenheit liegenden Perspektive, ohne Kenntnis der künftigen medizinischen Entwicklungen, aber auch der eigenen Situation einschließlich der höchstpersönlichen Wertvorstellungen, bereits eine definitive „Verfügung" über das eigene Schicksal (mit Bindungswirkung für den behandelnden Arzt!) verantworten lassen soll, bleibt unerfindlich und nur auf der Basis eines gänzlich inhaltsleeren Verständnisses von „Selbstbestimmung“ erklärlich, das der skrupulösen Haltung bei den todesgeneigten Willensbekundungen bzw. Handlungen im Falle des Suizids oder eines Verlangens i.S.d. \216 StGB (siehe o. 1., 2.) diametral widerspricht. In rechtlichen Sicherungen nur „unnötig hohe Anforde-

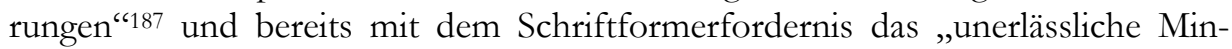

\footnotetext{
182 Bioethik-Kommission des Landes Rheinland-Pfalz (Fn. 65), S. 36: Die Garantie unantastbarer Würde des Menschen gebietet, „dass eine von ihm früher eigenverantwortlich getroffene Entscheidung auch dann noch respektiert wird, wenn er die Fähigkeit zu eigenverantwortlichem Entscheiden inzwischen verloren hat"; ebenso BGHZ 154, 205 (Leitsatz 1).

183 Zur mutmaßlichen Einwilligung sogleich im Text.

184 Dazu auch Schreiber, in: Medizinische Klinik, 2005, 429 ff.

185 Vgl. Duttge, Intensiv- und Notfallmedizin, 2005, 171 ff.; Preis der Freiheit (Fn. 58), S. 29 ff.

186 In diesem Sinne auch der AE-StB 2005, vgl. Schöch/Verrel, GA 2005, 553, 566, sowie das Gutachten zum 66. DJT, vgl. Verrel (Fn. 15), C 82

187 Schöch/Verrel, GA 2005, 553, 567; s.a. Verrel (Fn. 15), C 83: „überflüssiger Filter“.
} 
destmaß an Ernsthaftigkeits- und Missbrauchskontrolle“188 gewährleistet zu sehen (so der AE-StB), ist prinzipienvergessen allein vom gewünschten Resultat diktiert. Dabei sind dahingehende Risiken doch bekanntlich keineswegs auf Fälle der aktivdirekten Sterbehilfe beschränkt ${ }^{189}$. Ein Blick über die Grenzen auf das kürzlich in Kraft getretene österreichische Patientenverfügungsgesetz ${ }^{190}$ sollte daher in der „heißen Phase“ des derzeitigen rechtspolitischen Diskurses noch einmal zu bedenken geben, ob wahre Liberalität nichts anderes meint als bloßes Laissez-faire.

Die dritte Folgerung fordert schließlich die nötige Konsequenz, sobald von einer selbstbestimmten Willensbekundung des Patienten ausgegangen wird, d.h.: Die Reichweite von Patientenverfügungen trotz ihrer grundsätzlichen Anerkennung auf ein bestimmtes Stadium im Vorfeld des Todes (insbesondere: sobald das „Grundleiden einen irreversibel tödlichen Verlauf“" angenommen hat) begrenzen zu wollen ${ }^{191}$, widerspricht der eigenen Prämisse und lenkt die - durchaus berechtigte - Sorge vor Missbrauch und Autonomiedefiziten in falsche Bahnen ${ }^{192}$. Mit Recht betont demgegenüber der AE-StB, dass sich mit der Patientenautonomie das Recht verbindet, ,auf jede Behandlung und damit auch auf die Vornahme ärztlich indizierter und Genesung versprechender Maßnahmen zu verzichten“193. Dies gilt sogar in sachlicher Hinsicht, was der heftig umkämpften Qualifizierung der künstlichen Ernährung (PEG-Sonde) als „gewöhnliche“ oder „außerordentliche“ Behandlung die ihr über lange Zeit hinweg zugeschriebene Relevanz nimmt ${ }^{194}$ : Wenn nämlich jeder ärztliche Eingriff der (ggf. mutmaßlichen) Einwilligung bedarf, dann kann es in der Tat keine „unverzichtbare Basisversorgung“ geben ${ }^{195}$. In zeitlicher Hinsicht kommt dem Kriterium der Todesnähe allerdings eine ausschlaggebende Bedeutung hinsichtlich der zweiten - die „medizinische Indikation“ betreffenden - „Säule“ zu: Rechtlich anerkannt ist, dass in der sog. „Terminalphase“ lebenserhaltende Maßnahmen nicht mehr ,angezeigt“ sind. Allerdings besteht im medizinischen Umfeld offenbar keine rechte Klarheit über Maßstab und Kriterien einer solcherart „physiologischen futiliy“, die Juristen mit dem Begriff der „Aus-

\footnotetext{
188 Schöch/Verrel, GA 2005, 553, 566

189 Vgl. auch Birnbacher (Fn. 175), S. 358: „Die Gefahr der Insinuation besteht bei der passiven Sterbehilfe genauso und dürfte in der Praxis eine bedeutend größere Rolle spielen - nicht nur deshalb, weil Fälle der passiven Sterbehilfe weitaus häufiger sind, sondern auch, weil bei der Nahelegung eines Wunsches nach passiver Sterbehilfe eine sehr viel geringere Hemmschwelle zu überwinden ist".

190 Bundesgesetz über Patientenverfügungen (PVG) v. 8.5.2006, BGBl. I Nr. 55/2006 (abrufbar unter: www.ris.bka.gv.at/) (Stand: 03.09.2006); dazu näher Duttge, ZfL 2006, 3 (im Erscheinen) 191 In diesem Sinne bekanntlich die Enquete-Kommission des Deutschen Bundestages zu „Ethik und Recht der modernen Medizin“, Zwischenbericht: Patientenverfügungen v. 13.9.2004, BT-Drucks. 15/3700; ebenso kürzlich das Zentralkomitee der deutschen Katholiken (ZdK), berichtet in: KNA v. 30.6.2006; i.d.S. - wenngleich mit anderem Sinngehalt - auch BGHZ 154, 205, 215, der sich durch BGHSt 40, 257, 260 gebunden sieht.

192 Dazu näher Duttge u.a. (Fn. 58), S. 23 ff.; weiterhin in: Höfling (Fn. 20), S. 91 ff.

193 Schöch/Verrel, GA 2005, 553, 563

194 So bereits Duttge, GA 2005, 606, 609; wie hier auch Otto, NJW 2006, 2217, 2219

195 Zutreffend Schöch/Verrel, GA 2005, 553, 561
} 
sichtslosigkeit“ oder „Irreversibilität“ kennzeichnen ${ }^{196}$. Die derzeitige Lage ist um so prekärer, als in manchen Stellungnahmen und Berichten innerhalb des medizinischen und medizinethischen Diskurses unter einer „sinnlosen Lebenserhaltung“ mitunter ganz andere Fallgestaltungen verstanden werden, die cum grano salis wiederum Aspekte einer diffusen, u.U. auch durch ökonomische Erwägungen ${ }^{197}$ geprägten Bewertung nach „Lebensqualität“ zum Inhalt haben ${ }^{198}$. Deshalb lehnt der AE-StB zu Recht - und abweichend vom AE-Sterbehilfe 1986199 - auch bei Patienten im „persistent vegetative state“ (sog. apallisches Syndrom, Wachko$\mathrm{ma})^{200}$ einseitige Behandlungsbegrenzungen kategorisch $\mathrm{ab}^{201}$, allerdings allein wegen der diagnostischen und prognostischen Unsicherheiten sowie aufgrund des „fehlenden gesellschaftlichen Konsenses über den Umgang mit dieser Patientengruppe“; für im Wesentlichen zutreffend wird dagegen die Einschätzung gehalten, dass aufgrund der „objektiven Situation“ der „mutmaßliche Wille“ des Betroffenen in die Richtung einer Lebensbeendigung weise ${ }^{202}$.

Weder hier noch in sonstigen Fällen außerhalb der Terminalphase werden aber Umstände feststellbar sein, ,aus denen zuverlässige (!) Rückschlüsse auf die individuellen (!) Einstellungen und Präferenzen des Patienten gezogen werden können“"203. Mag die äußere Lage auch noch so hoffnungslos erscheinen: Stets ist es „das Gesetz des Lebens, das jedes Leben weiterleben will“204. Der frühere Vorsitzende Richter am Bundesgerichtshof Kutzer hat dieser Wendung treffend hinzugefügt: „Wenn die Gesellschaft solche Menschen nicht mehr ernähren will, ohne durch ein ausdrückliches oder wenigstens eindeutig interpretierbares Verlangen des Kranken dazu legitimiert worden zu sein, handelt sie nicht zum Wohle des Patien-

196 Vgl. etwa den Regelungsvorschlag des AE-StB ( 214 I Nr. 4 StGB-E): „,...handelt nicht rechtswidrig, wenn bei nahe bevorstehendem Tod im Hinblick auf den Leidenszustand des Betroffenen und die Aussichtslosigkeit einer Heilbehandlung die Aufnahme oder Fortführung lebenserhaltender Maßnahmen nach ärztlicher Erkenntnis nicht mehr angezeigt ist“; s.a. Verrel (Fn. 15), C 100: „Inkurabilität der Grunderkrankung“ und „unmittelbare Todesnähe“.

197 Z.B. Illhardt, in Heiß (Hrsg.), Kompendium Altersmedizin, 2005, S. 7: „Balance der Ressourcen“.

198 Weiterführende Überlegungen bei Duttge, NStZ 2006, 479 ff.

199 Vgl. $\int 214$ Abs. 1 Nr. 2 StGB-E, abgedr. in: Schöch/Verrel, GA 2005, 553, 557

200 Zum Krankheitsbild näher: Multi-Society Task Force on PVS, The New England Journal of Medicine 1994, 1499 ff.; Nacimiento, DÄ 1997, A-662 ff.

201 Schöch/Verrel, GA 2005, 553, 568 f.; dazu näher Simon, in: Höfling (Fn. 20), S. 103, 107 ff.

202 Entlarvend Thias, Möglichkeiten und Grenzen eines selbstbestimmten Sterbens durch Einschränkung und Abbruch medizinischer Behandlung, 2004, S. 194: „Bei irreversiblem Bewusstseinsverlust ... richtet sich ein objektiviert bestimmter (!) mutmaßlicher Patientenwille auf die Abstandnahme von weiteren lebensverlängernden Maßnahmen. ... [Demgegenüber konstituiert ein falsch verstandener Lebensschutz einen] ... menschlicher Selbstbestimmung (!) und Würde (!) zuwiderlaufenden Zwang zum Weiterleben".

203 Verrel (Fn. 15), C 90; Roxin (in: Medizinstrafrecht [Fn. 78], S. 99) stellt unter Verweis auf die neuere Judikatur fest, dass danach ,auch in solchen Fällen nötig ... [ist], nach konkreten Anhaltspunkten für den mutmaßlichen Willen zu forschen“.

204 Kutzer, in: Härlein u.a. (Hrsg.), Medizin und Gewissen - wenn Würde ein Wert würde, 2002, S. 165,167 
ten, sondern zu ihrem eigenen Wohl“'205. Die vom Zeitgeist getragene206 breite Zulassung nicht-freiwilliger Therapiebegrenzungen mittels der bei solcher Handhabung wahrlich „lebensgefährlichen“ Rechtsfigur der „mutmaßlichen Einwilligung“ sollte daher, nicht zuletzt auch angesichts der niederländischen Situation, dringend überdacht werden. John Harris hat das Problem in drastischen Worten als „Zeichen der Dekadenz einer Gesellschaft“ bezeichnet, „,wenn sie um jeden Preis die Wünsche leidender Menschen, die sterben wollen und gute Gründe dafür besitzen, vereitelt, während sie zugleich weiterhin zulässt, dass viele Menschen, die weiterleben wollen und deren Leben durch medizinische Eingriffe verlängert werden können, eines vorzeitigen Todes sterben“"207. Von größter Wichtigkeit ist daher, nicht zuletzt auch hinsichtlich der nötigen Akzeptanz der Grenzmarkierungen in der Gesellschaft, die Stimmigkeit und Folgerichtigkeit der einzelnen Wertentscheidungen innerhalb eines konsistenten Gesamtsystems zum Umgang mit menschlichem Leben in der letzten Lebensphase. Obgleich die de lege lata vorfindliche Sterbehilfetypologie mitnichten als Resultat eines vorausschauend durchdachten Systematisierungsprozesses bezeichnet werden kann, lässt sich ihr in der Sache (ggf. mit den vorstehenden Korrekturen) eine hohe Plausibilität nicht absprechen. Dass sich die aktuellen Reformbestrebungen im Kern (ungeachtet vereinzelter Klärungen im Detail) vor allem dahin verstehen, „den derzeitigen Rechtszustand transparenter zu machen“ (der mangelnden „Kommunizierbarkeit von Rechtsprechungskasuistik“" wegen) ${ }^{208}$, kann in diesem Lichte nicht mehr überraschen.

\section{Alternative Begrifflichkeiten?}

Gleichwohl häuft sich - wie schon eingangs notiert - die Kritik an der bereits seit längerem etablierten und vorstehend näher erläuterten Terminologie, die offenbar vielen nicht mehr als zeitgemäß gilt und auch in den „Grundsätzen“ der Bundesärætekammer keinen Niederschlag gefunden hat ${ }^{209}$. Im Vordergrund steht dabei die bekannte Schwierigkeit der Abgrenzung zwischen ,aktiver“" und „,passiver“ Sterbehilfe; jedoch wird auch der Begriff der ,,indirekten Sterbehilfe“ als nicht zielführend wie überhaupt schon der Oberbegriff als unangemessene Beschönigung und Verschleierung des Gemeinten betrachtet. In diesem Sinne findet sich erst un-

\footnotetext{
205 Kutzer, ebd.

206 Seltene Ausnahmen: Beckmann (Fn. 45), S. 212 ff.; Höfling, JuS 2000, 111, 116 f.; Schumann (Fn. 132), S. $70 \mathrm{ff}$.

207 Harris (Fn. 162), S. 32 (Einleitung zur deutschen Ausgabe); siehe auch S. 130 f.: „,...offensichtlich (!), dass diese Fälle für illegal erklärt werden sollten“.

208 Verrel(Fn. 15), C 56 und 60; siehe auch Schöch/Verrel, GA 2005, 553, 556: frühere Vorschläge „aktualisieren und fortschreiben, wobei der seitherigen Entwicklung in Rechtsprechung, Wissenschaft und Gesetzgebung Rechnung getragen wird (!)“.

209 Abgesehen von der dort ungenau benannten (verbotenen) ,aktiven Sterbehilfe“.
} 
längst in der Stellungnahme des Nationalen Ethikrats (,Selbstbestimmung und Fürsorge am Lebensende“ ${ }^{210}$ beanstandet, dass der Begriff „Hilfe ... positiv besetzt“ sei, also etwas signalisiere, das „legitim und begrüßenswert“ sei; diese „positive Färbung" werde jedoch im Kontext der ,aktiven Sterbehilfe" problematisch ${ }^{211}$. So mag es bei oberflächlicher Betrachtung in der Tat erscheinen; doch zeigt schon ein genauerer Blick auf die sonst vorfindliche Rechtsterminologie, dass die Verwendung des Begriffs „Hilfeleistung“ keineswegs auf den Bereich sozialadäquaten Verhaltens beschränkt ist (vgl. $\iint 27,257,259$ StGB). Im Übrigen hat die in Deutschland begegnende Scheu vor dem international geläufigen Sprachgebrauch („Euthanasie“) bekanntlich historische Gründe, was auch der Nationale Ethikrat nicht verkennt. In seinen eigenen Vorschlägen weist er zutreffend darauf hin, dass mit dem darüber hinaus in Betracht kommenden Terminus "Sterbebegleitung“ üblicherweise nur „Maßnahmen zur Pflege und Betreuung von Todkranken und Sterbenden“ bezeichnet werden, wozu „körperliche Pflege, das Löschen von Hunger- und Durstgefühlen, Mindern von Übelkeit, Angst, Atemnot, aber auch menschliche Zuwendung und seelsorgerischer Beistand“ zählen²12. Diese „pflegerische Sterbebegleitung "213 ist rechtlich geboten, im Lichte des Lebensschutzes jedoch unproblematisch, so dass mit diesem Begriff ohne Verwischung der Sachunterschiede nicht zugleich die problematischen - auf die Lebenserhaltung des Patienten (möglicherweise) Einfluss nehmenden - ärztlichen Entscheidungen in Bezug genommen werden können. Die Begriffsbildung sollte stets die spezifische Differenz zu benachbarten Sachverhalten kennzeichnen, auch wenn sich alle unter ein gemeinsames übergreifendes Thema fassen lassen ${ }^{214}$. In diesem Sinne besteht eine bedeutsame Divergenz zwischen „Sterbebegleitung“ und „Sterbehilfe“: Der Begleiter beschränkt seine Sorge auf die Begleitumstände des Sterbevorgangs, während der Sterbehelfer unmittelbar Einfluss nimmt ${ }^{215}$. Die Bezeichnung des neuen „Alternativ-Entwurfs Sterbebegleitung“ ist hingegen wohl eher der Abgrenzung zum AE-Sterbehilfe 1986 geschuldet; läge es anders ${ }^{216}$, so gewönnen die Bedenken hinsichtlich einer beschönigenden Begrifflichkeit hier erst an Überzeugungskraft. Wer daher, anders als offenbar der Nationale Ethikrat ${ }^{17}$, auf einen Oberbegriff nicht gänzlich verzichten mag, dem bleibt nur das Festhalten am Gebräuchlichen und Eingeführten.

\footnotetext{
210 Abrufbar unter: www.ethikrat.org/stellungnahmen/stellungnahmen.html (Stand: 03.09.2006)

211 Nationaler Ethikrat (Fn. 16), S. 26

212 Vgl. Nationaler Ethikrat (Fn. 16), S. 28

213 Bioethik-Kommission des Landes Rheinland-Pfalz (Fn. 65), S. $124 \mathrm{ff.}$

214 Sehr zu Recht betont von Beckmann, DRiZ 2005, 252

215 Von Pfäfllin (Recht \& Psychiatrie 2006, 75, 76) daher nicht unplausibel als „Sterbenachhilfe“ bezeichnet.

216 Für einen Wechsel auch des Obergriffs zugunsten der „Sterbebegleitung“ das Gutachten zum 66. DJT, vgl. Verrel (Fn. 15), C 118 (These 2).

217 Der Kritik (S. 26) folgt insoweit kein Alternativvorschlag (vgl. S. 28 f.).
} 
Dem Begriff der ,,indirekten Sterbehilfe“ wird demgegenüber vorgehalten, dass er die im Vordergrund stehende Schmerzbehandlung und Sedierung nicht zum Ausdruck bringe: „Es geht nicht um Hilfe zum Sterben, sondern um Therapien in der Sterbephase“218. Konsequent sprechen AE-StB und Juristentagesgutachten deshalb von „Leidensminderung“ bzw. „leidensmindernden Maßnahmen“, was zwar die erstrebte „Distanz zu strafbaren Patiententötungen“ schafft ${ }^{219}$, jedoch die (potentiell) nachteilige Einwirkung auf die Lebensdauer der Patienten im Unterschied zur Palliativmedizin verschleiert. Inkonsequent behält der AE-StB zudem den Begriff der ,aktiven direkten Sterbehilfe“220 bei, was die Möglichkeit einer „,indirekten“ Sterbehilfe bereits impliziert; Verrel wechselt dagegen folgerichtig zum Begriff der „Tötung auf Verlangen“ über ${ }^{221}$, der freilich die sachgedankliche Verknüpfung zu Krankheitszuständen, die überhaupt an eine „Sterbehilfe“ denken lassen (,aussichtsloser Zustand“, „unerträgliche Leiden“) 222, löst: Erbetene Euthanasie ist doch aber ,alles andere als eine bloße ,Tötung auf Verlangen' im Sinne des $\int 216$ StGB, ... [für die] jedes beliebige, subjektiv noch

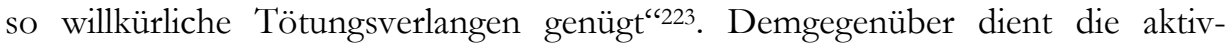
direkte Sterbehilfe nicht weniger als die ,indirekte“ allein der Behebung des „Leidens“" (,zielgerichtete Tötung zur Leidbeendigung“"224, allerdings auf eine (aus den vorstehend beschriebenen Gründen) ${ }^{225}$ nicht verantwortbare und daher von Rechts wegen auch nicht akzeptierte Weise. Der vom Nationalen Ethikrat für die erlaubte Form der Leidminderung vorgeschlagene Begriff der „Therapien am Lebensende" 226 ist dagegen schlechterdings nichts sagend.

Den Kern der Kritik am Terminologischen bildet jedoch schon seit langem die „passive Sterbehilfe“, der vorgehalten wird, den irrigen Anschein einer ,rein naturalistischen Unterscheidung" zur verbotenen aktiven (direkten) Sterbehilfe zu wecken $^{227}$. Zu Missverständnissen führt dies bekanntlich vor allem beim sog. „technischen Behandlungsabbruch“, der - vom Arzt oder einer in die organisatorischen Abläufe des Krankenhausbetriebes eingebundenen Person verübt ${ }^{228}$ unabhängig von dazu erforderlichen „Aktivitäten“ wie z.B. den Energieeinsatz

\footnotetext{
218 Nationaler Ethikerat (Fn. 212), Stellungnahme v. 13.7.2006, S. 27; s.a. Sahm, ZfL 2005, 45, 48

219 Verrel (Fn. 15), C 61

220 Schöch/Verrel, GA 2005, 553, 582

221 Verrel (Fn. 15), C 61; ebenso Nationaler Ethikrat (Fn. 16), S. 29

222 Vgl. Art. 2 Nr. 1 lit. b) des niederländischen Gesetzes über die Kontrolle der Lebensbeendigung auf Verlangen und der Hilfe bei der Selbsttötung v. 11.04.2001, abrufbar unter: www.aerzteblatt.de/ v4/plus/down.asp?typ=PDF\&id=1407 (Stand: 03.09.2006)

223 Geilen (Fn. 120), S. 24; siehe auch Beckmann, DRiZ 2005, 252, 253: „Das Opfer einer Tat gem.

\ 216 StGB kann kerngesund sein“; Kun₹, in: Donatsch (Hrsg.), Trechsel-FS 2002, S. 613, 615

224 Ingelfinger, ZfL 2005, 38, 39

225 Siehe o. 2

226 O. Fn. 218 , S. 28

227 Schöch/Verrel, GA 2005, 553, 560; Verrel (Fn. 15), C 56

228 Vgl. MK/Schneider (Fn. 78), Vor SS 211 ff. Rn 110
} 
beim Abschalten eines Reanimators de jure als Unterlassen („passive Sterbehilfe“) gewertet, im medizinischen Umfeld jedoch signifikant häufig (rechtsirrig) als (verbotene?) aktive Sterbehilfe verstanden wird ${ }^{229}$. Noch keineswegs vorentschieden ist damit jedoch die Frage, ob die Ursache hierfür tatsächlich im Begrifflichen liegt, also gar nicht adäquat zum Ausdruck kommt, was gemeint ist. Erste Zweifel an dieser These rühren aus dem Umstand, dass bei Kennzeichnung eines schädigenden Einwirkens mit dem Zusatz des „Aktiven“ außerordentlich nahe liegt, die Verhaltensmodalität des Unterlassens mit dem Kontradiktum des „Passiven“ zu versehen. Hinzu kommt aber vor allem, dass dieses Verständnis auch der Alltagsmoral alles andere als fremd ist: Man stelle sich etwa nur die Situation vor, am Morgen von seinem Nachbarn erwartungswidrig nicht gegrüßt zu werden. Ungeachtet aller möglichen „Aktivitäten“ bei der Begegnung geschieht die ehrenrührige Missachtung durch ein Unterlassen, der Nachbar bleibt insoweit „passiv“. In diesem Lichte würde sich die Veränderung der juristischen Begrifflichkeit somit vom Alltagsverständnis abkoppeln, ohne mit etwas Besserem aufwarten zu können. Denn die vor allem unter Philosophen beliebte Ersetzung der passiven Sterbehilfe

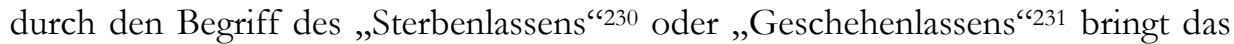
Einbezogensein des ,aktiven“ Behandlungsabbruchs gewiss nicht weniger undeutlich zum Ausdruck. Die anstelle dessen von der Bundesäratekammer bevorzugte „Änderung des Therapieziels“232 ist hochgradig anfällig für eine missbräuchliche Erweiterung des Anwendungsfeldes, weil sich hierunter ohne weiteres auch die aktive, gezielte Tötung subsumieren ließe. Die vom Gutachter zum Deutschen Juristentag favorisierte „Behandlungsbegrenzung “233 erfasst nur das Abstandnehmen von einer bereits eingeleiteten Maßnahme, nicht aber den von vornherein beschlossenen Verzicht hierauf ${ }^{234}$. Der AE-StB versucht sich deshalb mit einer umständlichen - Aufzählung der phänomenologisch vorstellbaren Modalitäten („Beenden, Begrenzen oder Unterlassen lebenserhaltender Maßnahmen“)235, wechselt damit aber vom Rechtlichen zum Faktischen und verliert infolgedessen den normativen Ausgangspunkt der Abgrenzung zwischen ,aktiver Tötung“ und „Unterlassen“ aus den Augen: Denn phänomenologisch besteht zwischen dem Behandlungsabbruch durch einen Arzt bzw. einen beliebigen Dritten kein Unter-

\footnotetext{
$229 \mathrm{Zu}$ den einschlägigen Umfrageergebnissen vgl. die Nachweise o. Fn. 17 f.; siehe auch Sahm, ZfL 2005, 45, 47: „Faktisch wird aktiv gehandelt“.

230 Guckes, Das Argument der schiefen Ebene. Schwangerschaftsabbruch, die Tötung Neugeborener und Sterbehilfe in der medizinethischen Diskussion, 1997, S. 146 ff.; so auch Nationaler Ethikrat (Fn. 16), S. 29

231 Birnbacher (Fn. 175), S. 344

232 Grundsätze der Bundesärztekammer zur ärztlichen Sterbebegleitung (Fn. 67), Ziff. II; zust. Sahm, ZfL 2005, 45, 48

233 Verrel (Fn. 15), C 60

$234 \mathrm{Zu}$ dieser Differenzierung bereits o. bei Fn. $18 \mathrm{f}$.

235 Schöch/Verrel, GA 2005, 553, 584
} 
schied; von letzterem erwarten wir aber keine lebenserhaltenden Maßnahmen ${ }^{236}$, sondern nichtschädigendes Verhalten gegenüber dem Patienten. Hieran gemessen ist das Einwirken auf fremde Rettungshandlungen nicht lediglich ein „Geschehenlassen“ (Unterlassen), sondern ,aktive“ Tötung ${ }^{237}$. Wer dies so sieht, sollte daher besser bei der bisherigen Terminologie bleiben und mit Nachdruck für ein zutreffendes Verständnis der weithin etablierten Typologie eintreten. Es spricht für sich, wenn der Nationale Ethikerat auch für die von ihm vorgeschlagenen Alternativbegriffe am Ende einräumt, dass hierdurch „die Schwierigkeiten, die es bereitet, medizinische Maßnahmen und Entscheidungen am Lebensende angemessen zu beschreiben, nicht restlos beseitigt" werden ${ }^{238}$. Anlass zur Veränderung besteht aber erst, wenn die Vorzüge des Neuen überwiegen.

\section{Ausblick}

Was nach alledem festzuhalten bleibt, ist die Erkenntnis, dass sich die Einzelheiten erst dann erschließen, wenn sie zum Ganzen in Beziehung gesetzt werden. Unser Streben nach ordnenden Schablonen steht immer in Gefahr, die Komplexität des Eigentlichen zu verkürzen. Umso dringlicher ist der Appell, fortwährend wieder den Über-Blick zu suchen, um uns nicht mit verengter Sicht im Labyrinth der Konkreta zu verirren. Die ärztliche Tätigkeit ist mehr als bloßes Handwerk; sie ist eine Kunst, die sich Leib und Seele, mit einem Wort: der Ganzheit des Menschen widmet. Weil dies nur im harmonischen Zusammenwirken des Arztes mit dem Patienten möglich ist, kommt dem Gedanken der Patientenautonomie nicht nur in ihrer Eigenwertigkeit, sondern auch als Grundbedingung für das Erreichen des erstrebten Heilungserfolgs eine herausragende Bedeutung zu. Nur möge niemand glauben, der Patient suche seinen Arzt auf, um ,sich selbst zu verwirklichen“"239. Er ist mehr als nur Konsument, die Medizin mehr als bloße Dienstleis-

\footnotetext{
236 Klassisch Geilen (Fn. 120), S. 22 Fn. 36: „Juristisch ist die Apparatur für den Arzt nichts anderes als sein verlängerter Arm“. - Der hierfür auch gebrauchte Begriff des „Unterlassens durch Tun“ (Roxin, in: Bockelmann [Hrsg.], Engisch-FS 1969, S. 380, 381 ff., 395 ff.) ist allerdings unnötig verwirrend und sollte besser vermieden werden.

237 So die h.M., vgl. etwa Kühl, Strafrecht Allgemeiner Teil, 5. Aufl. 2005, \18 Rn 18; MK/Schneider (Fn. 78), Vor $\int S 211$ ff. Rn 110; Schönke/Schröder/Lenckner/Eisele (Fn. 79), Vorbem. SS 13 ff. Rn 159; a.A. jedoch Gropp, in: Duttge u.a. (Hrsg.), Schlüchter-GS, 2002, S. 173, 179; Otto, Gutachten D zum 56. DJT, 1986, S. 43

238 Nationaler Ethikrat (Fn. 16), S. 29

$239 \mathrm{Zu}$ Recht betont von Schreiber, Anhörung vor dem Ausschuss für Rechts- und Verfassungsfragen des Niedersächsischen Landtages, 69. Sitzung v. 18.1.2006, S. 13: „Der Grund ärztlicher Behandlung ist, Leiden zu mindern und Leben zu verlängern"; siehe auch das Resümee des lesenswerten Buches von Tolmein, Keiner stirbt für sich alleine. Sterbehilfe, Pflegenotstand und das Recht auf Selbstbestimmung, 2006, S. 230: „Es ist ... kein guter Weg, einseitig das Selbstbestimmungsrecht von Patienten zu akzentuieren, wie es gegenwärtig in mehreren Gesetzentwürfen zur Regelung von Vorsorgevollmachten und Patientenverfügungen gemacht wird, aber die außerordentlich prekäre Situation und die enorme Abhängigkeit der Patienten in dieser Phase von Ärzten, von einer guten Therapie, von Hilfsmitteln, von ideeller Unterstützung und moralischem Zuspruch nicht zu berücksichtigen“".
} 
tung, das Leben mehr als eine Aneinanderreihung einzelner Erfolgs- und Glückserlebnisse. Der Tod gewinnt seinen Sinn erst durch das Leben, wie umgekehrt das Leben erst durch den Tod. „Wer Leben will, ohne zu sterben, wird am Ende sterben, ohne gelebt zu haben“" (Louis Evely). 


\title{
Selbstbestimmung bei Wachkoma und Demenz
}

\author{
Hilmar Prange
}

\section{Einleitung}

Vor einiger Zeit sollte an der Harvard Business School durch Schenkung von 20 Millionen Dollar ein Ethik-Lehrstuhl eingerichtet und ein entsprechender Vorlesungszyklus angeboten werden. Ein Gremium von Professoren stellte jedoch fest, dass man ohne vereinbarte absolute Werte nicht über Ethik reden kann. Die Spende wurde zurückgegeben. Die Financial Times kommentierte, dass „die Wurzel des Problems im Verlust des Glaubens an objektive Standards“ zu suchen sei.

Diese Einschätzung kann - jedenfalls in dieser Form - für die medizinischen Wissenschaften nicht zutreffend sein; denn das Vorliegen ethischer Maximen wird in der Heilkunst seit zweieinhalb tausend Jahren als verbindlich akzeptiert. Mag man nun auch die Intention und die Inhaltlichkeit des um 400 v. Chr. entstandenen Hippokratischen Eides zunehmend infrage stellen, allein die Existenz dieses Eides reflektiert schon die Bereitschaft der Ärzte, sich selbst ethische Normen zu geben und sich diesen auch zu unterwerfen. Freilich sind die meisten Einzelaussagen des Eides heute überholt. Man konnte sich einfach vor hundert oder gar vor zwei tausend Jahren nicht vorstellen, dass es eines Tages Intensivstationen geben

1 Hahne P (2005): Schluss mit lustig. Ende der Spaßgesellschaft. Verlag Johannis, Lahr 
wird, auf denen zumindest in Einzelfällen das Sterben verhindert aber ein eigenbestimmtes Leben nicht wieder hergestellt werden kann. Auch haben sich infolge des Faktischen bestimmter gesellschaftlicher Entwicklungen viele medizinethische Wertungen verändert. Die Pervertierung des Hippokratischen Eides im Dritten Reich und in der DDR ${ }^{2}$ förderte zudem die kritische Distanz zu dieser überbrachten ethischen Normierung. Konsequenterweise beschloss der Weltärztebund im Jahre 1949 das Genfer Gelöbnis, welches die Kernpunkte des Hippokratischen Eides übernimmt, jedoch die Ehrfurcht vor dem Leben ganz in den Mittelpunkt ärztlichen Handelns stellt. Die medizinische Entwicklung ging indes weiter. Allein schon durch verbesserte Behandlungsmöglichkeiten stieg die Zahl von Patienten im Wachkoma, ebenso solcher mit Demenz, Locked-in-Syndrom und sog. Minimally Conscious State.

Man muss also an dieser Stelle ernsthaft die Frage stellen, ob solche Zustände wie das Wachkoma ein Danaer Geschenk der modernen Medizin sind. Auf den ersten Blick scheint dies der Fall zu sein. So erbrachten Nachuntersuchungen bei Personen, die während eines Krankenhausaufenthaltes wegen Kreislaufstillstand reanimiert werden mussten, dass lediglich 6\% dieser Patienten den Kreislaufstillstand und die anschließende ,erfolgreiche“ Reanimation ohne erhebliche neurologische Defizite überstanden. Ein etwas größerer Teil der Behandelten verblieb im Wachkoma ${ }^{3}$.

Nach vorsichtigen Schätzungen werden in Europa jährlich 375.000 Personen wegen kardiopulmonaler Zwischenfälle reanimiert ${ }^{4}$. Wenn auch nur $6 \%$ davon ohne nennenswerte neurologische Dauerschäden gerettet werden und ein eigenbestimmtes Leben führen können, so profitieren in jedem Jahr 22.500 Personen uneingeschränkt von der Reanimationsmaßnahme. Angesicht solcher Zahlen soll und darf der Sinngehalt der kardiopulmonalen Reanimation nicht hinterfragt werden. Dem trägt die Konvention für den Schutz. der Menschenrechte und -würde in Biologie und Medizin ${ }^{5}$ von 1996 voll und ganz Rechnung: „When because of an emergency situation the appropriate consent cannot be obtained, any medically necessary intervention may be carried out immediately for the benefit of the health of the individual concerned" (Artikel 8: Notfallsituation).

Das Prinzip der Patientenautonomie, welches in den letzten Dekaden einen dominierenden medizinethischen und rechtlichen Stellenwert erhielt, ist dabei

\footnotetext{
2 Weil F (2006): Ärztliche Ethik mit neuem Inhalt gefüllt. Vom Eid des Hippokrates zur moralischen Verpflichtung eines sozialistischen Arztes. Deutsches Ärzteblatt, 103, B1361-1364

3 Skrifvars MB, Rosenberg PH, Finne P, Halonen S, Hautamäki R, Kuosa R, Niemelä H \& Castrén M (2003): Evaluation of the in-hospital Utstein template in cardiopulmonary in secondary hospitals. Resuscitation, 56, 275-282

4 De Vreede-Swagemakers JJ, Gorgels AP, Dubois-Arbouw WI, van Ree JW, Daemen MJ, Houben LG \& Wellens HJ (1997): Out-of-hospital cardiac arrest in the 1990`s : a population-based study in the Maastricht area on incidence, characteristics and survival. J Am Coll Cardiol, 30, 1500-1505

5 Council of Europe (1996): Convention for the Protection of Human Rights and Dignity of the Human Being with Regard to the Application of Biology and Medicine. Dir/Jur, (96)14. Strasbourg
} 
durchaus berücksichtigt. Es wird unterstellt, dass der Patient die Reanimation will - jedenfalls so fern keine anderen Informationen vorliegen. Das Problem der dadurch zunehmenden Zahl von Personen im Wachkoma versucht man durch Optimierung der Standards für die kardiopulmonale Reanimation ${ }^{6}$ und Verbesserung der Nachsorge der Betroffenen zu reduzieren. Die vermeintliche Unvereinbarkeit zwischen Wachkoma und Patientenautonomie ist damit allerdings nicht aufgehoben.

\section{Terminologische Aspekte}

\subsection{Koma, Wachkoma, Minimally Conscious State}

Unter Koma (synonym: Bewusstlosigkeit) versteht man das Fehlen geistiger Wahrnehmung der Umgebung und seiner selbst - im Englischen „total loss of perception of environment and self“، Ein Komatöser hat praktisch immer geschlossene Augen. Diese werden weder spontan noch auf Anruf oder Schmerzreize geöffnet; Aufforderungen werden nicht befolgt. Bewegungen auf Schmerzreize oder spontan sind im Koma möglich?

Dem davon abzugrenzenden Wachkoma (synonyma: Coma vigile, apallisches Syndrom, persistierender vegetativer Status) liegt eine globale Funktionsminderung der Großhirnrinde (Kortex) zugrunde, wobei das aufsteigende „Wecksystem" der im Hirnstamm lokalisierten Formatio reticularis intakt sein kann. Die Patienten sind nach Abklingen der Akutphase des Schädigungsereignisses wieder wach, öffnen phasenweise die Augen, die ins Leere blicken. Psychischer Kontakt lässt sich nicht herstellen. Typischerweise zeigen die Patienten spontanes oder reflexartiges Augenaufschlagen und Atmen. Manchmal scheinen sie mit geöffneten Augen wach zu sein; dabei imponiert mitunter ein plötzliches Augenrollen. Zu anderen Zeiten sind die Augen geschlossen; die Patienten scheinen zu schlafen. Sie können auf schmerzhafte und außergewöhnliche Reize die zuvor geschlossenen Augen öffnen und die Atmung beschleunigen; Herzfrequenz und Blutdruck steigen an und gelegentlich zeigen sich eine einfache Mimik und Bewegungen. Letztere schließen Kauen, Zähneblecken und Schlucken ein. Unvermittelt oder aufgrund von nicht sprachlichen Geräuschen beobachtet man Reaktionen wie

6 Adams HA, Baumann G, Cascorbi I, Dodt C, Ebener C, Emmel M, Geiger S, Jansens U, Klima U, Klippe HJ, Knoefel WT, Lampl L, Marx G, Müller-Werdan U, Pape HC, Piek J, Prange H, Roesner D, Roth B, Sarrafzadeh A, Standl T, Teske W, Unterberg A, Vogt PM, Werner GS, Windolf J, Zander R, Zerkowski HR \& Flemming A (2006): Kardiopulmonale Reanimation - CRP. Eine Empfehlungen der Interdiziplinären Arbeitsgruppe Schock der Deutschen Interdisziplinären Vereinigung für Intensivmedizin und Notfallmedizin zur praktischen Umsetzung der Richtlinien des European Resuscitation Council 2005. Intensivmedizin und Notfallmedizin, 43, 446-451

7 Haupt WF, Firsching R, Hansen HC, Horn M, Janzen RWC, Neuen-Jacob E, Prange HW, RudolfJ \& Zschocke S (2000): Das akute postanoxische Koma: Klinische, elektrophysiologische, biochemische und bildgebende Befunde. Intensivmed, 37, 597-607 
Greinen, Grunzen, Ächzen, leises Schreien oder Lächeln. Eine kaum reproduzierbare leichte Kopf- oder Augenbewegung in Richtung neuer Geräusche oder optischer Reize ist möglich. Diese Fähigkeiten - vermutlich subkortikalen Ursprungs können auch bei Kindern mit sog. Anenzephalie, deren Wachzustand ohne Bewusstheit erhalten ist, beobachtet werden ${ }^{8}$. Tetraparese und pathologische Bewegungsmuster wie Streck- und Beugespasmen und vegetative Störungen gehören zum Syndrom des Wachkomas. Der Neurologe verifiziert es klinisch mittels bestimmter ,archaischer" Reflexmechanismen, die durch Wegfall der kortikalen Hemmung auftreten, sowie durch die elektrophysiologische Funktionsdiagnostik der Hirnrinde.

Ein Wachkoma ist nicht immer irreversibel. Namentlich nach traumatischer Hirnschädigung kann innerhalb von 12 Monaten, ausnahmsweise auch später, der Zustand durch Wiedererlangen kortikaler Funktionen überwunden werden.

Es gibt bei schwer Hirngeschädigten auch Zustände, die nicht dem Wachkoma zuzuordnen sind, weil zeitweise eine bewusste Reaktion des Patienten zu induzieren ist. Vom American Congress of Rehabilitation Medicine ${ }^{9}$ wurde 1995 der Begriff des „minimal responsive state“ kreiert, der später durch Minimally Conscious State (MCS) ersetzt wurde. Gemeint sind Personen, die durch krankhafte Prozesse oder deren Folgen unfähig sind, Anweisungen korrekt zu befolgen oder zu kommunizieren, die aber unregelmäßig, jedoch wiederholt, deutliche Zeichen von Bewusstheit gegenüber der Umgebung oder ihrer selbst aufweisen. Solche Personen können immer mal wieder visuelle Fixierungen und gefühls- oder bewegungsmäßige Reaktionen zeigen, die unabhängig von der Anwesenheit besonderer gravierender Reize erfolgen, wie z.B. Phasen des Weinens, die ausschließlich durch Stimmen von Familienangehörigen hervorgerufen werden, Befolgen von Anweisungen, Greifen von Gegenständen sowie gestische oder sprachliche Ja-NeinAntworten.

Dem MCS wird als Unterkategorie der akinetische Mutismus ${ }^{10}$ zugeordnet. Er ist gekennzeichnet durch schwere Einschränkungen jeglicher Bewegungsabläufe, des Sprechens und Denkens ohne gleichzeitige Störungen der Wachphase. Spontaneität, Initiative und Spontanbewegungen sind nahezu vollständig verloren. Sprache, Emotionen und Vorstellung - soweit überhaupt vorhanden - sind erheblich reduziert. Das Fehlen von endogen gesteuertem Verhalten lässt es zu, dass

\footnotetext{
8 Faymonville M-E, Pantke K-H, Berré J et al. (2004) : Zerebrale Funktionen bei hirngeschädigten Patienten. Was bedeuten Koma, „vegetative state“, „minimally conscious state“, Locked-in-Syndrom und Hirntod? Anaesthesist, 53, 1195-1202

9 American Congress of Rebabilitation Medicine (1995): Recommendation for use of uniform nomenclature pertinent to patients with severe alterations of consciousness. Arch Phys Med Rehabil, 76, 205209

10 Cains H, Oldfield RC, Pennybacker JB \& Witteridge D (1941): Akinetic mutism with an epidermoid cyst of the third ventricle. Brain, 64, 273-290
} 
die Aufmerksamkeit passiv auf beliebige Umweltreize gerichtet ist, denen der $\mathrm{Pa}$ tient ausgesetzt wird ${ }^{11}$.

Akinetischer Mutismus und MCS sind in der Regel Durchgangszustände hin zur normalen Bewusstheit nach Schädel-Hirn-Traumen und Reanimationen (upgrading) oder hin zum Wachkoma bei fortschreitenden Krankheiten des Zentralnervensystems (ZNS) wie z.B. der Creutzfeldt-Jakob-Krankheit (downgrading).

\subsection{Locked-in-Syndrom ${ }^{12}$}

Dem Locked-in-Syndrom liegt definitionsgemäß eine querschnittsartige funktionelle oder substantielle Unterbrechung der Bahnverbindungen vom Großhirn zum unteren Hirnstamm und zum Rückenmark (und damit zum gesamten Körper) in der ventralen Brückenhälfte zugrunde (Abb. 1). Wir sprechen von einer motorischen De-Efferenzierung des Gehirns. Typischerweise bleiben die Haubenregion mit Formatio reticularis und okulomotorischem Apparat erhalten. Es resultiert eine komplette Lähmung aller unterhalb der Schädigung liegenden Muskelaktivitäten des Körpers, Kopfes und Gesichts. Die Patienten sind sprech- und bewegungsunfähig mit Ausnahme von (vertikalen) Augenbewegungen. Über diese Augen- oder Lidbewegungen kann mittels eines eingeübten Codes mit den Patienten kommuniziert werden; denn bei den Betroffenen sind Wachzustand, Bewusstheit und Denken und damit die Funktion der Hirnrinde (zumeist) unbeeinträchtigt. Ein Locked-in-Syndrom ist reversibel, wenn es durch Krankheiten der peripheren motorischen Nerven hervorgerufen wird. Liegt eine Durchblutungsstörung der Brücke zugrunde, dann ist ein Wiedererlangen der motorischen Funktionen unwahrscheinlich aber nicht ganz unmöglich.

11 Faymonville M-E, Pantke K-H, Berré J et al. (2004), vgl. Anm. 8

12 Plum F \& Posner JB (1983): The diagnosis of stupor and coma. Saunders, Philadelphia 


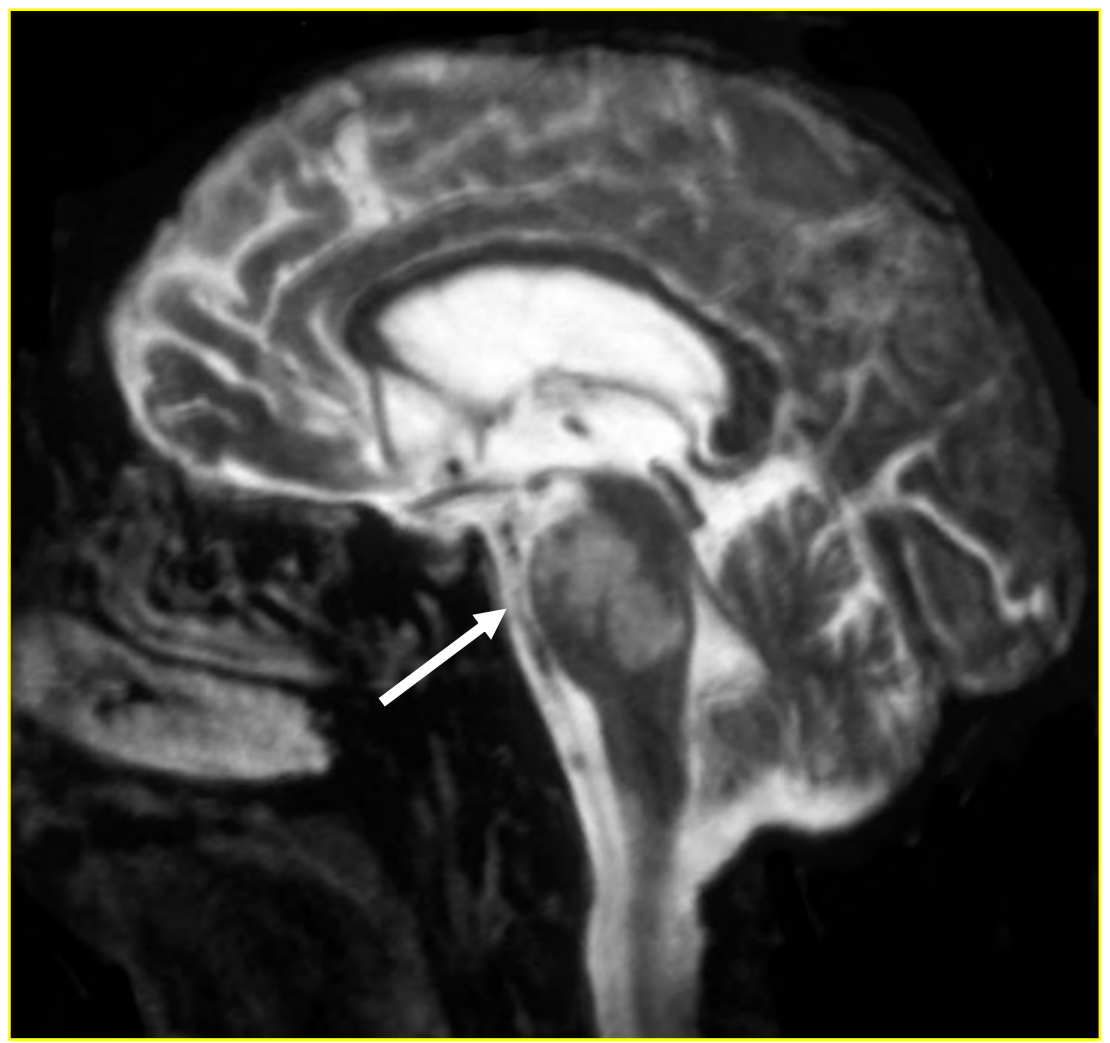

Abb.1: Zentrale Pons-Nekrose nach Thrombose der Basilararterie. Durch unverzügliche medikamentöse Auflösung des Thrombus wurde der Zelluntergang im gesamten Hirnstamm verhindert; die Strukturen der zentrale Ponsregion (Brücke; Pfeil) konnten nicht erhalten werden.

Im nachfolgenden Fallbericht aus DIE ZEIT beschreibt ein Angehöriger die Befindlichkeit eines Patienten mit Locked-in-Syndrom ${ }^{13}$ :

Für R. wurde das Liegen auf den immer gleichen Stellen, selbst bei regelmäßiger Umbettung schon eine Qual, durch Nacblässigkeit des Pflegepersonals verschlimmert. Jeden Tag kam seine Frau in die Klinik, aber sie konnte nicht immer dort sein. „Wenn ich später wiederkam, lag er immer noch so da. "Niemand, der dem schwitzenden Patienten im Sommer eine dünnere Decke gegeben bätte. Niemand, der ibm den Scbweiß von der Stirn wischte, damit er nicht in das ständig offene Auge floss. Niemand, der die Fliegen verscheuchte. Niemand, der das Radio abgestellt hätte, das die Put_frau nach ibrer Arbeit

13 Leicht $\mathrm{R}$ (2005): Das letzte Tabu. Die ZEIT, 44, 27.10.2005 
einfach anließ. R. mochte Jazz. und klassische Musik, die Putzfrau den Dudelsender WDR 4. „Das ist Folter", sagt Frau R....

\subsection{Demenz}

Ein Demenz-Syndrom kann diagnostiziert werden, wenn intellektuelle Störungen in mehreren Bereichen nachweisbar sind und der Schweregrad dieser Störung zu einer erheblichen Beeinträchtigung in der Bewältigung der Alltagsaufgaben führt. Die intellektuellen Störungen betreffen 1. das Gedächtnis und damit das Aufnehmen und Wiedergeben neuer Informationen sowie den Verlust früher erlernter und vertrauter Inhalte; 2. das Denkvermögen - nämlich den Ideenfluss, die Informationsverarbeitung und die Fähigkeit zum rationalen Urteilen; 3. Wesensart und Verhalten mit Störungen der Impulskontrolle, des Sozialverhaltens und der Motivation. Der dritte Punkt tritt gerade im mittleren und späteren Krankheitsverlauf in den Vordergrund.

Alltagspraktische Fertigkeiten wie Waschen, Ankleiden, Essen, persönliche Hygiene sowie die Kontrolle der Körperausscheidungen einschließlich Toilettenbenutzung sind schließlich nicht mehr möglich. Der Betroffene ist im fortgeschrittenen Stadium voll pflegebedürftig und in keiner Weise entscheidungsfähig.

Dementielle Syndrome nehmen vom 65. Lebensjahr an mit zunehmendem Alter in ihrer Häufigkeit exponentiell zu. Das Risiko einer 65-jährigen Frau, in der ihr noch verbleibenden Lebenszeit an einer Demenz zu erkranken, liegt bei 34,5 Prozent, das eines gleichaltrigen Mannes bei 16 Prozent $^{14}$. Die häufigsten Demenzursachen sind Alzheimer Demenz (60 \%), vaskuläre Demenz (15-20\%), LewyKörper-Demenz (1-15\%), fronto-temporale Demenz (Morbus Pick $5 \%$ ), Morbus Parkinson (1\%), Creutzfeldt-Jakob-Erkrankung (1\%) und diverse andere Ursachen (ca. $5 \%$ ). Die Zuordnung in kortikale und subkortikale Demenz folgt kausalen Gesichtspunkten. Bei der kortikalen Demenz (z.B. Alzheimer- und fronto-temporale Demenz) sind schon früh verbales und visuelles Gedächtnis, Sprache und visuell-räumliche Verarbeitung gestört. Subkortikale Demenzen (z.B. bei M. Parkinson und Multipler Sklerose) werden durch verminderte Motivation, Aspontaneität bis hin zur Apathie, Erschöpfbarkeit, psychomotorische Verlangsamung und affektive Indifferenz auffällig.

14 Ott A, Breteler MM, van Harskamp F, Stijnen T \& Hofman A (1998): Incidence and risk of dementia. The Rotterdam Study. Am J Epidemiol, 147, 574-580 
Tafel 1: Demenzdefinition der Arzneimittelkommission der Deutschen Ärzteschaft in Anlehnung an ICD $10^{15}$

Die Störungen von 1 und 2 müssen schwer genug sein, um eine wesentliche Beeinträchtigung der Aktivitäten des täglichen Lebens nach sich zu ziehen. Dauer: mehr als sechs Monate

\section{Störungen des Gedächtnisses}

Aufnahme und Wiedergabe neuerer Informationen

Verlust früher erlernter und vertrauter Inhalte (in späteren Stadien)

\section{Störungen des Denkvermögens}

Störung der Fähigkeit zum vernünftigen Urteilen

Verminderung des Ideenflusses

Beeinträchtigung der Informationsverarbeitung

\section{Störungen der emotionalen Kontrolle \\ Störung des Sozialverhaltens \\ Störung der Motivation}

\section{Zur Frage der Personalität}

Der Wissenschaftliche Beirat der Deutschen Ärztekammer kommentierte 1982 die Definition des Hirntodes mit folgendem Satz: „Mit dem Organtod des Gehirns sind die für jedes personale menschliche Leben unabdingbaren Voraussetzungen, ebenso aber auch alle für das eigenständige körperliche Leben erforderlichen Steuerungsvorgänge des Gehirns endgültig erloschen."16

Zweifelsfrei ist eine Selbstbestimmung nicht mehr möglich, wenn das personale menschliche Leben erlischt. Dieses Erlöschen ist indes ein Prozess, der sich durchaus über längere Zeiträume als eine Stunde oder einen Tag erstrecken kann. Wo liegt aber die Grenze?

Autonomie und Personalität sind inhaltlich und kausal eng verknüpfte Begriffe. Die Attribution des Personalitätsbegriffes lässt sich am besten anhand seiner Entwicklung darlegen. Das lateinische Wort personare (durchtönen) hat seinen Ursprung im antiken Theater: Der Schauspieler ließ Individualität - nämlich ein selbständiges, einmaliges, unverwechselbares Wesen - durch die Maske, hinter der er sich verbarg, durchklingen. Der spätantike Philosoph Boetius (480-524) kons-

\footnotetext{
15 Arzneimittelkommission der deutschen Ärz̧teschaft (2004): Demenz, Empfehlungen zur Therapie der Demenz. 3. Aufl. Arzneiverordnung in der Praxis

16 Stellungnahme des Wissenschaftlichen Beirates der Bundesäratekammer (1982): Kriterien des Hirntodes. Deutsches Ärzteblatt, 79, 45-55
} 
tatierte Persona est naturae rationalis individua substantia, was sich mit „Person ist der unteilbare Selbstand eines geistigen Wesens“ übersetzen lässt.

Descartes (1596-1650) definierte personales Leben in seiner Philosophie des methodischen Zweifeln dahingehend, dass unbezweifelbar nur ist, dass ich bin, weil ich denke. - Also Denken und Zweifeln als wesentliche Merkmale der Personalität?

Bei Kant (1724-1804) impliziert das Person-Sein die Fähigkeiten zur sittlichen Selbstbestimmung, zur Selbstgesetzgebung, zum Erkennen und vernünftigen Handeln, außerdem die Würde sowie die Selbstzweckhaftigkeit.

Durchaus von Kantianischen Ansätzen ausgehend ${ }^{17}$ entwickelte der Utilitarismus im 20. Jahrhundert eine normative Theorie, die auf dem Prinzip Nutzen als moralisches Kriterium beruht. An letzterem soll die Richtigkeit und Falschheit von Handlungen sowie Recht und Unrecht gesellschaftlicher Normen und Institutionen gemessen werden. Namentlich bei Peter Singer ${ }^{18}$ ist die Empfindungsfähigkeit eine Grundlage der utilitaristischen Weltsicht, wobei nichtmenschliche Lebewesen in die moralische Berücksichtigung eingeschlossen werden. Singer sieht damit keinerlei moralische Bevorzugung der Spezies Mensch und setzt den „Speziesismus“ dem Rassismus gleich. Er vertritt die Auffassung „...ist ein Wesen nicht leidensfähig oder nicht fähig, Freude und Glück zu erfahren, dann gibt es nichts zu berücksichtigen." Normale erwachsene Menschen hätten allerdings geistige Fähigkeiten, deretwegen sie unter gewissen Umständen mehr leiden als Tiere. „... Was dieses Argument betrifft, so gehören nichtmenschliche Lebewesen, Säuglinge und schwer geistig behinderte Menschen zur selben Kategorie.“ Der Utilitarismus erkennt zwar die Würde des Menschen an, sieht allerdings das Allgemeinwohl, welches sich aus dem Wohl der Einzelnen zusammensetzt, als höchstes Ziel. Im Alltag würden bei Entscheidungen ständig Vor- und Nachteile für verschiedene Personen größenmäßig verglichen. Alles Tun und Lassen habe sich am Wohle des Menschen zu orientieren. Grundsätzlich wiege aber das Wohl der Vielen mehr als das Wohl des Einzelnen. Dem von Singer vertretenen Präferenzutilitarismus zufolge ist eine Handlung, die der Präferenz irgendeines Wesens entgegensteht, ohne dass diese Präferenz durch entgegengesetzte Präferenzen ausgeglichen wird, moralisch falsch. Wenn also das Tötungsverbot gegen die Präferenz des Betroffenen steht, dann wäre es moralisch falsch. Euthanasie wäre erlaubt, wenn es der Betroffene wünscht.

Bei solchen Erörterungen verliert der Personalitätsbegriff seine Attribution. Nach Singer ist für die Zuerkennung des Lebensrechtes - wie erwähnt - nicht Zugehörigkeit einer biologischen Gattung, sondern allein der Grad an Selbstbewusstsein, Vernunftgebrauch und die Fähigkeit zur Zukunftsplanung, den ein Lebewesen erreicht, ausschlaggebend ${ }^{19}$. So könne die Tötung eines Affen (z.B. im

17 Cornman JW (1975): Perception, common sense, and science. New Haven, Yale University Press

18 Singer P (1993): Praktische Ethik. 2. Aufl. Reclam, Stuttgart

19 Singer $P$ (1993), vgl. Anm. 17 
Rahmen von Tierversuchen) schwerer wiegen als die Tötung eines schwer geistig behinderten Menschen ${ }^{20}$.

Das Komitee für Medizinische Ethik der Britischen Medizinischen Assoziation (BMA) definiert das Wachkoma in den BMA Guidelines on Treatment Decisions for Patients in a Persistent Vegetative State wie folgt ${ }^{21}$ :

- Intakter Schlaf-Wach-Rhythmus

- Erhaltene Funktionen des autonomen Systems von Hypothalamus und Hirnstamm

- Fehlen von reproduzierbaren zielgerichteten oder willkürlichen Reaktionen auf visuelle, akustische taktile oder noxiöse Reize

- Fehlendes Bewusstsein für das Selbst und die Umgebung

- Fehlende Hinweise für eine mentale Aktivität, Kognition, sprachliches Verständnis und sprachliches Ausdrucksvermögen

Ist damit das personale Leben einer Person im Wachkoma erloschen? Der Begriff des Teilhirntodes kam in den 80iger Jahren des 20. Jahrhunderts auf und bezog sich sowohl auf den „kortikalen Tod“ bei Wachkoma als auch auf den „Hirnstammtod“ nach schwersten Schädelhirntraumen. Den Begriff des Teilhirntodes hat man allerdings mehrheitlich aufgrund fehlender kollektiver Akzeptanz (und ethischer Absurdität) abgelehnt, er wurde jedoch kürzlich journalistisch wieder aufgegriffen: „...Zusätzlich ist aber an die Fälle zu denken, in denen der Hirntod schon vollends oder der Teilhirnhirntod („Wachkoma“) eingetreten ist, die Vitalfunktionen des Körpers aber mit maschineller Unterstützung noch länger aufrecht erhalten werden können..." 22 .

Die christlichen Kirchen begründen die Personalität mit der Gottebenbildlichkeit des Menschen; er behält auch im Falle absoluter Pflegebedürftigkeit seine Individualität und Unverwechselbarkeit. Im christlichen Verständnis ist die Würde des Menschen gerade deshalb unantastbar, weil sie nicht in den Leistungen oder der Leistungsfähigkeit des Menschen, sondern in der Beziehung Gottes zu jedem einzelnen Menschen begründet ist ${ }^{23,24}$.

\footnotetext{
20 Siehe auch Schockenhoff E (1993): Ethik des Lebens. Ein theologischer Grundriß. MatthiasGrünewald-Verlag, Mainz, S. 48

21 British Medical Association (BMA) (1994): Guidelines on Treatment Decisions for Patients in a Persistent Vegetative State. London

22 Leicht R. (2005), vgl. Anm. 13

23 Huber W (2002). Pflege und Ethik,. www.ekd.de/senioren/huber_020626_pflege_und_ ethik.html (Stand: 26.09.2006)

24 Sozialprinzipien der Katholischen Kirche (2004), www.studienkolleg-obermarchtal.de/ unterricht/sozialprinzipien_der_katholische.htm (Stand: 26.09.2006)
} 


\section{Kardinalfragen zum Lebensende}

Ehe wir uns nochmals dem Problempunkt „Autonomie des Patienten“ zuwenden, soll auf die Fragen danach, wie Personen mit Wachkoma oder Demenz sterben und wann ein Mensch mit Demenz oder Wachkoma überhaupt sterben darf, eingegangen werden.

Wie schon früher erwähnt, können Patienten mit Wachkoma und Demenz bei guter Pflege und Versorgung längere Zeiträume, auch Jahre, überleben. Häufige Todesursachen bei Demenz sind sich aufpfropfende Alterskrankheiten wie Schlaganfälle, Herzinfarkte, Herzversagen aus anderer Ursache, Tumorleiden und auch Unfälle - z.B. intrakranielle Blutungen als Sturzfolge (Abb. 2). Des Weiteren führen bei Demenzpatienten, insbesondere aber bei Personen im Wachkoma, sog. interkurrente Krankheiten zum Tode. Zumeist handelt es sich um Komplikationen, die sich aus der hochgradigen Pflegebedürftigkeit ergeben. Hierzu gehören rezidivierende Lungenentzündungen und aufsteigende Harnwegsinfekte mit konsekutiver Sepsis, infizierte Lagerungsgeschwüre, Thrombosen und Lungenembolien, Bolustod (unglückliches Verschlucken) sowie Entgleisungen des Wasser- und Elektrolyt-Haushaltes. Fast von selbst stellt sich hier die Frage danach, wie weit bei solchen Komplikationen eine Behandlung zu gehen hat.

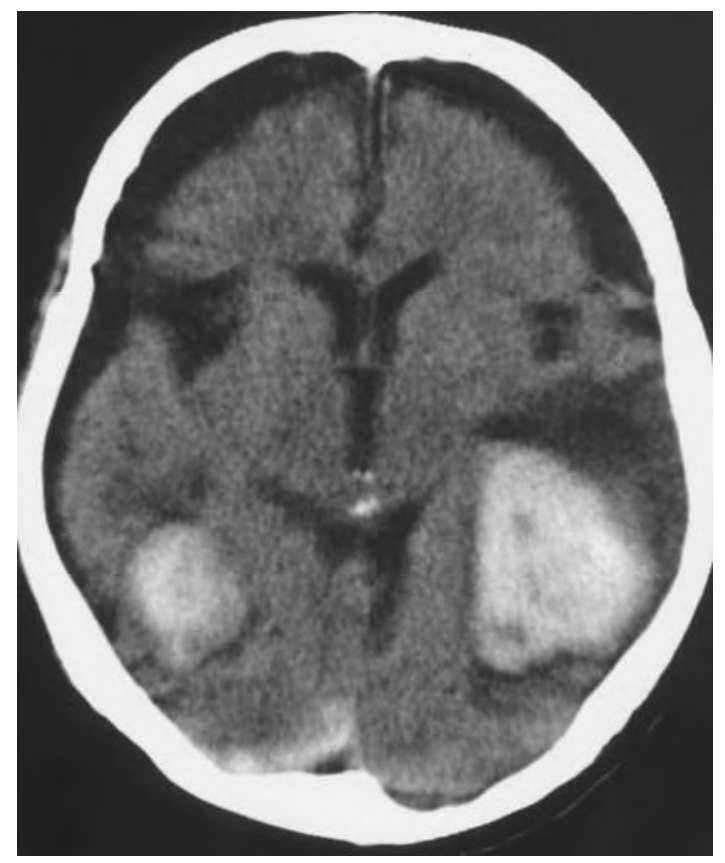

Abb. 2: Beidseitige Gehirnkontusionen nach Sturzereignis(sen) bei 72-jährigen Patienten mit Demenz vom Alzheimer-Typ; zusätzlich beidseitige subdurale Hygrome, offensichtlich als Folge früherer Stürze. 
Der Moraltheologe E. Schockenhoff nimmt dazu wie folgt Stellung:

Grundsätzlich darf jede Therapie zurückgenommen oder ganz abgebrochen werden, wenn sie ibr ursprüngliches Behandlungsziel nicht mehr erreichen kann. Medizinische Maßnabmen, die in keiner Weise mehr der Heilung, sondern nur noch der Verbinderung des Sterbens dienen, in dem sie den bereits verfügten Tod künstlich hinauszögern, fallen nicht mehr unter das Gebot des Lebensschutzes. ${ }^{25}$

Papst Pius XII führte bezogen auf eine ähnliche Konstellation aus:

Der Mensch kann (soll) keine medizinischen ... Maßnahmen an sich vornehmen lassen, die zwar eine Behebung schwerer physischer oder psychischer Beschwerden ... bewirken, gleichzeitig aber die dauernde Auslöschung oder eine dauernde Herabminderung der freien Selbstbestimmung, d.h. der menschlichen Persönlichkeit in ibrer typischen und charakteristischen Funktion bewirken; die den Menschen also zu ... einem lebenden Automaten degradieren. ${ }^{26}$

Nochmals Schockenhoff:

Wo die Grundkrankheit längst zum sicheren Tod geführt hätte und die begonnene Therapie nur noch unnötige Leiden verursacht, gebietet vielmehr die Achtung vor der Würde des Sterbenden ibren Abbruch. ${ }^{27}$

Gilt dieser allseits konsensfähige Satz auch für Personen im Wachkoma, mit schwerer Demenz oder gar im Locked-in-Syndrom? Menschen im Wachkoma verdanken zweifelsfrei der modernen Medizin ihre Existenz. Ein Therapieabbruch ist in Fällen, für die der mutmaßliche Wille unbekannt ist, nicht grundsätzlich unethisch - aber der vielerorts geäußerte Wunsch nach medizinethischen Leitlinien für solche Fälle blieb unerfüllt.

Unlängst wurde eine Studie aus Kanada publiziert, nach der bei 19,5 \% von 851 Patienten chirurgischer und internistischer Intensivstationen die Therapie durch Abstellen der Beatmung beendet wurde. Bis auf 6 Überlebende verstarben früher oder später alle nach der Diskonnektion der Atemschläuche. Einer multivariaten Analyse zufolge waren 4 unabhängige Prediktoren mit der Beendigung der Beatmung assoziiert: 1. Gabe von Katecholaminen (vereinfacht: schwere Kreislaufprobleme), 2. Prognose des leitenden Arztes, dass die Überlebenswahrscheinlichkeit unter $10 \%$ liegt, 3. Prognose des leitenden Arztes, dass der Patient einen Monat nach Verlegung von der Intensivstation wegen kognitiver Defizite nicht das Krankenhaus verlassen können wird, 4. Einschätzung des leitenden Arztes, dass der Patient keine lebenserhaltenden Maßnahmen wünscht ${ }^{28}$. Bei kritischer

25 Schockenhoff E (1993), vgl. Anm. 19

26 HerKorr 7; 1952/53, 73; zitiert nach SchockenhoffE (1993), vgl. Anm.19

27 SchockenhoffE (1993), vgl. Anm. 19

28 Cook D, Rocker G, Marshall J et al. (2003): Withdrawal of mechanical ventilation in anticipation of death in the intensive care unit. New Engl J Med, 349, 1123-1132 
Durchsicht dieser Studie kommt der Verdacht auf, dass die Ärzte, insbesondere die Verantwortlichen sich vorzugsweise von eigenen, auf die Patienten projizierten Präferenzen leiten ließen.

Zweifelsfrei muss bei solchen Konstellationen die Entscheidungsgrundlage immer vom Einzelfall ausgehen. Auf keinen Fall darf jedoch der Arzt vom Heilenden und Lindernden zum Tötenden werden. Also selbst wenn extreme Ausnahmesituationen vorliegen, darf das Tötungsverbot nicht aufgehoben werden eingedenk der Worte von Horst Köhler (Bundespräsident) „nicht durch die Hand eines anderen sollen die Menschen sterben, sondern an der Hand eines anderen. "29 Auch aus diesem Grunde lehnen wir die Maximen des Präferenzutilitarismus ab, die eine Euthanasie auf Wunsch moralisch möglich machen.

Die Aufweichung dieses Standpunktes ist unterdessen vielerorts Realität. Eine anonymisierte europaweite Studie über „End-of-Life Practices“ auf europäischen Intensivstationen erbrachte, dass in 2,2 \% der Todesfälle von Intensivpatienten eine aktive Euthanasie ohne Einverständnis oder Aufforderung durch die Patienten vollzogen wurde - obwohl dies in allen beteiligten Ländern illegal ist ${ }^{30}$.

Die Einstellung deutscher, intensivmedizinisch tätiger Neurologen zu den Fragen Therapieabbruch sowie aktiver und passiver Sterbehilfe wurde von uns in einer Fragebogenaktion untersucht ${ }^{31}$. Im Erhebungsbogen stellten wir die Kasuistik eines Patienten mit Basilaristhrombose, Lysetherapie und verbleibendem Locked-in-Syndrom den Fragen mit Entscheidungsalternativen voran. Von 93 Ärzten aus allen Altergruppen wurde der Fragebogen ausgefüllt und zurück geschickt. Nur $58 \%$ wollten die Therapieentscheidungen detailliert mit dem wachen aber kaum reaktionsfähigen Patienten besprechen; mit den Angehörigen wollten auch nur $87 \%$ solche Gespräche führen. Eine passive Sterbehilfe (unter definierten Bedingungen durch Therapieabbruch) hielten $99 \%$ für ethisch vertretbar. Die Frage „Halten Sie grundsätzlich eine Lebensverkürzung durch aktive Sterbehilfe (durch aktive lebensverkürzende Maßnahmen) in besonders ausgewählten Einzelfällen für ethisch vertretbar?" wurde von $19 \%$ bejaht. Wohlgemerkt - es ging hier nicht um einen vom Patienten oder Betreuer gewünschten oder geforderten Therapieabbruch.

Diese publizierten Studien reflektieren die Unsicherheit der kurativ tätigen Ärzte im Umgang mit dem Lebensende - unabhängig davon, ob eine Willensbekundung des Patienten vorliegt oder nicht. Erhebliche Entscheidungshilfe für den Betreuer und den behandelnden Arzt bringen freilich Patientenverfügung oder vergleichbare rechtlich verbindliche Festlegungen (Vorsorge- und Betreuungs-

\footnotetext{
29 zitiert nach Drieschner F (2005): Ein Mann der dringend sterben wollte. DIE ZEIT, 44, 27.10.2005

30 Sprung CL, Cohen SL, Sjokvist P et al. (2003): End-of-Life practices in European intensive care units. The ethics study. JAMA, 290, 790-797

31 Thiel A, Schmidt H, Prange H \& Nau R (1997): Die Behandlung von Patienten mit Thrombosen der Arteria basilaris und Locked-in-Syndrom. Ein ethisches Dilemma. Nervenarzt, 68, 653-658
} 
vollmachten) mit sich. Sie sollten allerdings zu einem Zeitpunkt erstellt sein, an dem der Betroffene noch eigenbestimmt und entscheidungsfähig war.

Der Nationale Ethikrat (2006) führt zu diesem Punkt aus:

[K]ann ein Patient seinen Willen nicht äußern, ist auf frühere Willenäußerungen des Patienten zurück.zugreifen. Besteht eine Patientenverfügung, kann damit eine eindeutige, rechtlich erheblich Willensäußerung vorliegen. Feblt es an ibr oder bestehen Zweifel an ihrer Geltung für die aktuelle Entscheidungslage, so kann versucht werden, auf den mutmaßlichen Willen des Betroffenen abzustellen. Daraus wird allerdings der Aræzt keine binreichend sichere Entscheidungsgrundlage gewinnen können. Dazu bedarf es vielmehr zusätzlicher Verfahren der Abwägung und Entscheidungsfindung. Können auch dann nicht alle Zweifel ausgeräumt werden, hat die Erhaltung des Lebens Vorrang. Diesen Grundsatz stets zu beachten, ist auch gegen die Gefabren missbräuchlichen Verhaltens beim Sterben lassen eine wirksame V orsorge. .... Allerdings sollte für strafrechtliche und berufsrechtliche Sanktionen kein Raum sein, wenn eine medizinische Behandlung unter Abwägung ihrer Aussichten auf Erfolg, des Leidenszustandes des Patienten und seiner voraussichtlichen Lebenserwartung nicht mehr angezeigt ist und sie deshalb unterlassen, begrenzt oder beendet wird... ${ }^{32}$

\section{Zur Patientenautonomie}

Da niemand verpflichtet ist, zur Erhaltung von Leben und Gesundheit alle erdenklichen Maßnahmen anzuwenden, zumal wenn diese mit erheblichen Einschränkungen von Kommunikationsfähigkeit und Lebensqualität verbunden sind, muss der Arzt den Entschluss eines entscheidungsfähigen Patienten zum Therapieverzicht respektieren ${ }^{33}$. Dieser eher allgemein gehaltene Satz ist wohl für ärztliche Tätigkeiten in kurativem und palliativem Bereich hilfreich, er löst aber keinesfalls alle sich aus der Praxis ergebenden Probleme; hierfür ein Beispiel:

Kasuistik 1: A.I. v. B., $67 \mathrm{~J}$.

Der früher als Aræ̨t tätige 67-jährige Mann leidet unter einer Motoneuronerkrankung, die nach und nach zu einer schlaffen Lähmung der Beine, dann auch der Arme führte. Schließlich bestand das typische Syndrom einer amyotrophischen Lateralsklerose. Die Funktionsstörungen entwickelten sich allerdings sehr langsam. Der Patient war auch bei fortgeschrittener Bebinderung noch lange Zeit selbständig bzw. wenig pflegebedürftig. Die Familie, insbesondere die Ehefrau, übernahm zunehmend seine Pflege und Versorgung. Sie war jetzt auch die gerichtsamtliche Betreuerin. Wegen verschiedener Komplikationen (vor allem Pneumonien) wurden wiederholte Krankenhausaufenthalte notwendig, in letzter Zeit zunehmend.

32 Nationaler Etbikrat (2006): Selbstbestimmung und Fürsorge am Lebensende. Stellungnahme. 13. Juli 2006. Berlin

33 SchockenhoffE (1993), vgl. Anm. 19 
Nach nunmehr 10-jährigem Krankheitsverlauf ist Herrn I. schließlich komplett gelähmt (,plegisch"). Die Bewegung der Beine entspricht Kraftgrad 0/5; der Arme ebenfalls 0/5. An den Hände sind minimale Muskelkontraktionen (Kraftgrad 1/5) noch erkennbar. Sprechen ist seit längerem nicht mehr möglich; lediglich ein leichtes Lächeln kann man bei Ansprache des Patienten wabrnehmen. Schlucken ist beeinträchtigt; Pneumonien häufen sich. Formal entspricht sein Zustand weitgehend dem eines Lockedin-Syndroms. Ernährt wird der Herr I. über eine PEG-Sonde. Zur besseren Lungenpflege hat man jetzt ein Tracheostoma angelegt. Die Kommunikation erfolgt über ein sog. Eye-Gaze-Gerät: Durch Bewegung der Augen setzt der Pat. einen Buchstabendurchlauf in Gang, der durch Augenbewegung auch gestoppt werden kann. Auf diese Weise werden Worte zusammengesetఇt, Wünsche mitgeteilt, schließlich auch Briefe und Gedichte geschrieben.

Unmittelbar vor und nach der Tracheotomie (Kehlkopfschnitt) entstanden die beiden folgenden Gedichte:

20. September [...] (Brief an die Ehefrau zwei Tage vor dem Kehlkopfschnitt):

Der Rest ist Qual, die Hustenzabl verdirbt den Morgen, große Sorgen.

Der Todeskampf hat längst begonnen, das Ende nabt, es ist bald aus.

Das Leben ist gar bald zerronnen, jetat weiß ich nicht mehr ein und aus.

Gib bald mir Medizin zum schlafen, wir können's beide kaum noch schaffen. ich bitt, verzeih, was ich verschuldet, wir haben schon sehr viel erduldet, ich liebe dich so wie du mich.

Tausend danke schön, auf wieder sehn.

Es handelt sich zweifellos um ein Abschiedsgedicht, aus dem man Resignation und vielleicht auch Lebensüberdruss entnehmen könnte.

Zwei Tage nach der Tracheotomie haben sich Stimmung und Einstellung zum Weiterleben geändert:

24. September $[\ldots]$

Wenn Atmung nur noch Qual bedeutet, der Schnitter naht, zur Ernte läutet, dann wähl die Tracheotomie, sie wird fein helfen - aber, wie.

Trachea wird durch Saugen frei.

Dann Atemarbeit Kinderei.

Auf denn, es sei, ich bin bereit. 
Den Husten bin ich wirklich leid.

Ein lichtes grün der Wände begrüßt mich im OP.

Die Schwestern, etwas dunkler, ich still und freundlich seh.

Ich brauch nicht lange warten,

man schiebt mich nach Saal acht,

dort heißt es lange warten,

ich dichte, was gedacht.

Narkose blockt die Unterschrift.

Die Gattin sich ins Auto wirft,

nach dem sie angerufen ist,

ein Missverständnis, schöner Mist.

Sie eilt herbei und unterschreibt,

mir gibt sie dadurch Herzensfreud.

Die Gedichte belegen unserer Auffassung nach eindrucksvoll die Situationsabhängigkeit des Wunsches nach Weiterleben bei Kranken bzw. schwer Pflegebedürftigen. Die Befindlichkeit zum Zeitpunkt der Willensbekundung bestimmt also wesentlich die Entscheidung für oder auch gegen Weiterleben. Die Maxime der ärztlichen Ethik bleibt indes Voluntas aegroti - also dem Wunsch des Betroffenen folgen.

Ist der Arzt damit nur ein Dienstleister? Diese Frage muss entschieden verneint werden. Patientenautonomie kann nur realisiert werden, wenn der autonome Patient ausreichende und glaubwürdige Information über seine eigene physische Situation besitzt. Diese kann einzig und allein vom beratenden bzw. behandelnden Arzt kommen. Nicht hilfreich sind unselektierte Informationen, wie sie beispielsweise das Internet, manchmal auch sog. Selbsthilfegruppen ausgeben. Sie verstören oft Patienten und/oder Betreuer und führen häufig zu Entscheidungen, die irrational oder sogar für den Patienten schädlich sind. In DIE ZEIT ${ }^{34}$ wird mit recht ausgeführt „,...die Kunst, die letzte Verantwortung des Arztes in einem Gesetz hinweg zuregeln, hat noch keiner erfunden."

\section{Assistierter Suizid}

Die ethische Bewertung des Suizids, über die selbst im Nationalen Ethikrat keine übereinstimmende Position erreicht werden konnte, soll im Folgenden nicht Gegenstand der Erörterung sein. Auch möchten wir auf das Tun und Lassen des Notarztes beim Suizidversuch nicht eingehen. Unter dem Gesichtspunkt der Patientenautonomie soll die Frage danach, wie weit ein „Bilanzsuizid“, also ein Suizid, der von einem unheilbaren Kranken in freier Verantwortung und nach ernsthafter Erwägung seiner Perspektiven angestrebt wird, von einer dritten Person,

34 Leicht R (2005), vgl. Anm. 13 
im Zweifelsfall dem Arzt, unterstützt werden darf, angesprochen werden. Dafür möchten wir ein fiktives, aber sehr Praxis bezogenes Fallbeispiel vorstellen:

Kasuistik 2: T. S. 42 J

Der 1953 geborene Zahnarzt bemerkte im Alter von 32 Jahren erstmals passager auftretende Doppelbilder. Zu diesem Zeitpunkt betrieb er erfolgreich eine eigene Praxis. Zwei Jahre später stellten sich Taubheit in den Oberschenkeln und bald auch Schwäche bei längeren Gehstrecken ein. Mit fluktuierender Ausprägung manifestierte sich dann eine Harninkontinen ₹. Die neurologische Diagnostik (Liquoranalytik, Kernspintomographie und evozierte Potentiale) deutete mit hoher Wahrscheinlichkeit auf das Vorliegen einer Multiplen Sklerose hin. Der weitere Verlauf gestaltete sich wie folgt:

1989 spastische Paraparese, Inkontinenz, Rollstuhlpflichtigkeit; Ehefrau und Kinder verlassen ibn - offensichtlich nach heftigen familiären Zerwürfnissen.

1990 Paraesthesien der Hände und Arme, Ataxie, ausgeprägter Tremor; gezielte Bewegungen sind nur eingeschränkt möglich.

1992 Bettlägerigkeit, massiver Adduktorenspasmus mit konsekutivem Dekubitus an den Unterschenkelvorderseiten (Abb. 3a). Erneut kommt es zu sich wiederholenden Lungenentzündungen, Knochenverletzungen durch Stur: aufsteigenden Harnwegsinfekten und Dekubitalulzera (Abb. 3b).

1993 zunehmende Augenmuskel-Lähmungen mit Doppelbildern. Pat. ist nunmehr völlig pflegebedürttig; Suizidversuch mit Flunitrazepam (Robypnol®).

Ab 1994 ist der Pat. unfähig zu lesen und zu essen. Selbst die Lieblingsbeschäftigungen der letzten Jahre - Rauchen und Telefonieren - sind nur noch mit Unterstützung durch andere Personen möglich. Zu diesem Zeitpunkt wünscht T. S. eine Beratung darüber, auf welche Weise ein Suizid erfolgreich durchgeführt werden kann. Er legt sich ein Insulin-Depot an.

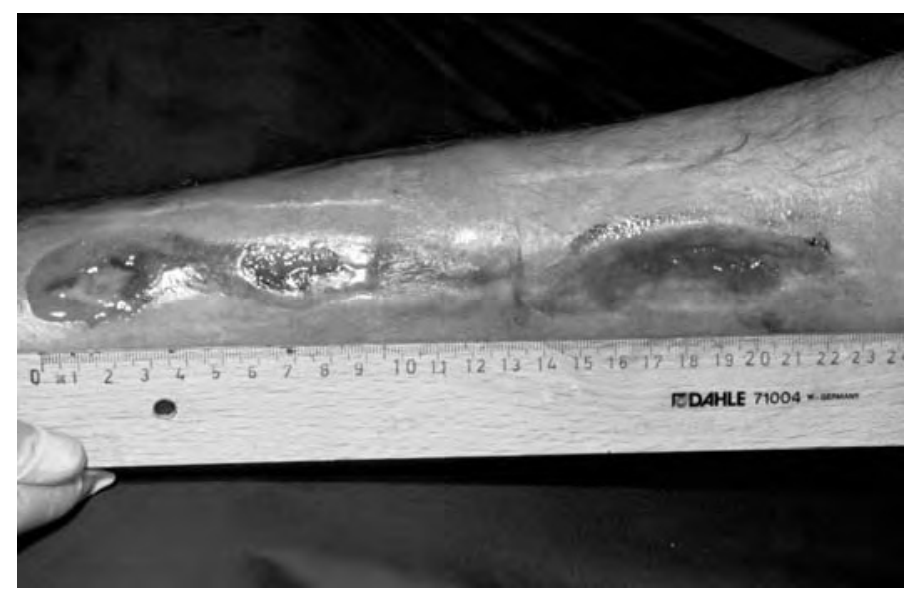

Abb. 3a: Dekubitalulzera an der Schienbeinvorderkannte durch massiven Adduktorenspasmus 


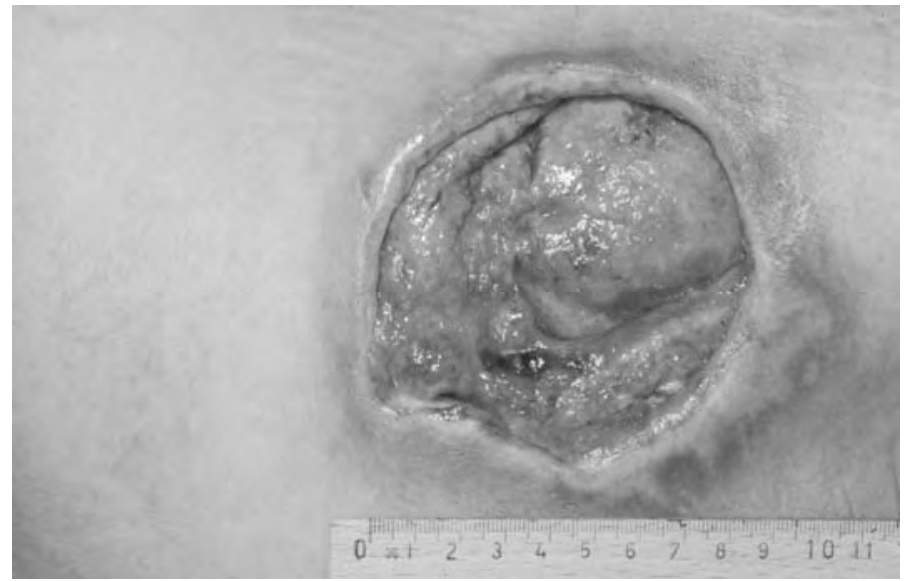

Abb. 3b: Ausgeprägtes Ulkus im Steißbereich bei total pflegebedürftigem MPatienten mit Multipler Sklerose

Im Mai 1995 verstirbt er im bypoglykämischen Schock böchstwahrscheinlich infolge einer Insulinverabfolgung.

Strittig ist hier schon, ob es in einer solchen Situation eine Rettungspflicht gibt. Der Nationale Ethikrat empfiehlt „Zurückhaltung“, die allerdings für den Arzt aufgrund seiner Garantenpflicht nicht unproblematisch sei. Jedenfalls meint die überwiegende Mehrheit des Nationalen Ethikrates, es solle bei der Straflosigkeit der individuellen Beihilfe zur Selbsttötung bleiben. Nach gegenwärtiger Rechtslage handelt straffrei, wer einem freiverantwortlichen Suizidenten ein weniger grausames Mittel verschafft, ihn also unterstützt - allerdings nur, solange er nicht Tatherrschaft übernimmt.

Die Bundesärztekammer vertritt die Ansicht, Suizidhilfe widerspreche dem beruflichen Auftrag des Arztes. Die Bereitstellung von Mitteln für den Suizid sei mit dem ärztlichen Ethos unvereinbar.

Dem ist nach der von uns mitgetragenen Auffassung folgendes entgegenzuhalten:

1. Ärztliche Beihilfe beim oben beschriebenen „Bilanzsuizid“ ist mit dem Gebot, zum Wohle des Patienten zu handeln, vereinbar.

2. Ärztlicher Beistand im Suizid kann dazu beitragen, dass der Patient im Suizid nicht mehr als notwendig leiden muss und dass ein von niemandem gewollter Zustand schwerer Schädigung (z.B. schwere hypoglykämische Hirnschädigung bei obigem Beispiel) statt des Todes eintritt.

3. Die Beurteilung der Entscheidungsfähigkeit und die Nachvollziehbarkeit der Entscheidung des Patienten kann noch am besten von Ärzten gewährleistet werden. 
4. Für manchen Patienten kann allein die Gewissheit, im Falle des Suizids mit der Hilfe eines Arztes rechnen zu können, Kraft und Mut zum weiteren Ertragen seines Leidens bringen.

5. Wenn wir die Autonomie des Patienten wirklich erst nehmen, dann können wir konsequenterweise nicht den von ihm gewünschten „Bilanzsuizid“ ausklammern.

Mehrere Bedingungen müssen dabei erfüllt sein:

a) Der Unterschied zwischen aktiver Sterbehilfe und assistiertem Suizid muss für den unterstützenden Arzt klar und eindeutig sein.

b) Der Arzt darf nicht Tatherrschaft übernehmen und sollte vielleicht auch nicht der bisher Behandelnde sein.

c) Der Arzt darf keinen ökonomischen Vorteil davon haben und darf auf keinen Fall erpressbar werden.

d) Der Arzt muss die Suizidbeihilfe als persönliche Gewissensentscheidung vollziehen und auch die Verantwortung dafür übernehmen.

Vorgenanntes ist nur realisierbar, wenn gesellschaftliche Akzeptanz hierfür besteht.

\section{Schlussfolgerungen}

Eine Selbstbestimmung am Lebensende ist bei Patienten mit Wachkoma und schwerer Demenz nicht möglich. Um dennoch die Autonomie eines Patienten bzw. einer Patientin zu verwirklichen, bedarf es einer Patientenverfügung oder einer vergleichbaren rechtlich verbindlichen Festlegung (Vorsorge- oder Betreuungsvollmacht). Sie sollte konkret und differenziert, schriftlich und zeitnah sein, wobei die Forderung nach Zeitnähe den Verhältnissen anzupassen ist: Eine Aktualisierung kurz vor dem Schädigungsereignis oder -prozess ist ausgesprochen wünschenswert. Da allerdings nur $10 \%$ der Bevölkerung 35 eine Patientenverfügung unterschrieben haben, ist dieses Hilfsmittel zur Realisierung der Patientenautonomie in den meisten Fällen nicht verfügbar. Die dann noch mögliche Ermittelung des mutmaßlichen Patientenwillens ist in der Praxis sehr verbreitet; aber der Grad der Zuverlässigkeit liegt erheblich unter dem einer Verfügung. Viele darauf explorierte Angehörige verstehen den Begriff des mutmaßlichen Patientenwillens nicht und glauben ihre persönliche Meinung ist gefragt oder sie projizieren eigene Erfahrungen und Emotionen in den mutmaßlichen Patientenwillen. In solchen Fällen entsteht für den verantwortlichen Arzt ein Dilemma, weil er nur eine Entscheidung zwischen zwei gleich unangenehmen Möglichkeiten vollziehen kann: Den wenig zuverlässigen Äußerungen der Angehörigen oder der eigenen aus ärztlicher Vernunft und Erfahrung hergeleiteten Vorstellung darüber, was der Patient

35 Jachertz N, Klinkhammer G \& Richter-Kublmann EA (2005): Mutmaßlicher Wille, weitreichende Folgen. Deutsches Ärzteblatt, 102, B996-999 
sich jetzt wohl wünschen würde, zu folgen. Letzteres kann kaum noch mit der Begrifflichkeit der Patientenautonomie vereinbart werden.

Anders ist die Situation beim Locked-in-Syndrom und dem Minimally Conscious State (MCS). Bei diesen Patienten kann der eigene Wille erfragt werden, auch wenn dies manchmal etwas aufwendig ist, weil Wiederholungen, Verständigungs-Codes, oft auch die Hilfe von Angehörigen notwendig werden.

Grundsätzlich hat der Arzt, der eine Patientenverfügung vorfindet, diese ebenso zu berücksichtigen wie den verbindlichen Wunsch eines artikulationsfähigen Kranken, eine leidensverlängernde Heilbehandlung abzubrechen.

Es bleiben viele ungeklärte Fragen, die von der Ärzteschaft zu tragen sind. Der Wunsch nach mehr Rechtssicherheit für Ärzte und betreuende Pflegekräfte wird nicht zu unrecht artikuliert. Dessen ungeachtet trifft folgender Satz zu: „Der Wunsch des Arztes, jedes mögliche Haftungsrisiko zu umgehen, kann auch den Gedanken der medizinischen Aufklärung pervertieren - wenn diese nämlich in einen ,bürokratisch perfektionierten Selbstschutz der Ärzte' verwandelt wird, der das Behandlungsrisiko wieder ganz auf den Patienten zurückverlagert. “36

36 Flöhl R (1982): Der Gesundheitsanspruch des Patienten und seine Grenzen. In: Martini GA (Hrsg): Medizin und Gesellschaft: ethische Verantwortung und ärztliches Handeln. Stuttgart, S. 161 


\title{
Rechtliche Grundlagen der Entscheidung über den Einsatz lebenserhaltender Maßnahmen
}

\author{
Volker Lipp
}

\section{Aktuelle Diskussion}

Aus der schlichten, ethisch oder religiös motivierten Forderung nach einem menschenwürdigen Sterben ist eine Fülle schwieriger Rechtsprobleme entstanden, um deren Lösung in Praxis, Wissenschaft und Gesetzgebung intensiv gerungen wird. Dabei ist mittlerweile ein zentraler Gesichtspunkt allgemein anerkannt: Die grundrechtlich geschützte Patientenautonomie umfasst auch das Recht, sich für oder gegen eine lebenserhaltende ärztliche Behandlung zu entscheiden ${ }^{1}$. Jedoch sind Bedeutung und Tragweite der Patientenautonomie für die so genannte „Sterbehilfe" heftig umstritten.

1 Vgl. nur Taupits, Empfehlen sich zivilrechtliche Regelungen zur Absicherung der Patientenautonomie am Ende des Lebens?, Gutachten A zum 63. Deutschen Juristentag 2000, A 11; Lipp, DRiZ 2000, 231 ff.; Spickhoff, NJW 2000, 2297 ff.; Berger, JZ 2000, 797 ff.; zum grundrechtlichen Schutz der Patientenautonomie: BV erfGE 52, 131 (168, 173 ff.); 91, 1 (29 ff.); Hufen, NJW 2001, 849 (851ff.); zur Anerkennung des Selbstbestimmungsrechts des Patienten im Zivil- und Strafrecht vgl. nur RGZ 68, 431 (433 ff.); BGHZ 29, 46 (49, 54); 106, 391 (397 f.); RGSt 25, 375 (381 ff.); 11, 111 (114); 32, 366 (378). Kritisch aber z.B. Duttge, Preis der Freiheit, 2004, S. 6 ff.; zur rechtspolitischen Diskussion insgesamt: Lipp, Patientenautonomie und Lebensschutz - Zur Diskussion um eine gesetzliche Regelung der „Sterbehilfe“, Göttingen 2005. 
Bereits vor geraumer Zeit haben die Strafgerichte die Einstellung lebenserhaltender Maßnahmen auf Wunsch des Patienten („passive Sterbehilfe“)2 für grundsätzlich zulässig erklärt, ebenso wie die medizinisch gebotene und mit Einwilligung des Patienten erfolgende Gabe von Schmerzmitteln oder anderen Medikamenten, die als Nebenwirkung das Leben des Patienten verkürzen („,indirekte Sterbehilfe ${ }^{\text {( }) ~} 3$. Danach konzentrierte sich die Diskussion darauf, ob und wenn ja, unter welchen Voraussetzungen ein Vertreter die Einstellung lebenserhaltender Maßnahmen verlangen kann, und auf welche Weise jemand Vorsorge bezüglich der ärztlichen Behandlung treffen kann, etwa durch Patientenverfügungen oder Vorsorgevollmachten ${ }^{4}$.

Hierzu sind in den letzten Jahren gleich mehrere obergerichtliche Entscheidungen ergangen, die Bewegung in die Debatte gebracht haben.

Gegenstand gleich zweier Verfahren war der Fall einer Zeugin Jehovas. Sie hatte vorsorglich sowohl bei der Aufnabme ins Krankenhaus als auch in einer früher verfassten Patientenverfügung erklärt, sie lehne eine Bluttransfusion ab. Als wegen einer Komplikation überraschend eine Bluttransfusion notwendig wurde, bestellte das Vormundschaftsgericht (im Folgenden: Vorm G) ihren Ehemann zum Betreuer der bewusstlosen Patientin. Dieser war kein Mitglied der Zengen Jehovas und stimmte der Bluttransfusion zu. Die $V$ erlängerung der Betreuung lehnte das Vorm $G$ ab, weil es mittlerweile erfabren hatte, dass ein Bevollmächtigter vorhanden war. Die Beschwerde gegen die Betreuerbestellung blieb wegen Erledigung obne Erfolg. Das BVerfG nabm die hiergegen eingelegte Verfassungsbeschwerde mangels Erfolgsaussicht nicht an. Das VormG habe die Grundrechte der Patientin nicht verletrt, weil es die Vollmacht nicht gekannt habe. Außerdem sei es um eine Entscheidung über Leben und Tod gegangen 5 . Die Schmerzensgeldklage gegen die Klinik und die behandelnden Ärzte wies das OLG München Anfang 2002 ebenfalls ab6. Die Patientenverfügung sei nicht verbindlich, maßgeblich sei vielmehr die Entscheidung des Ehemanns als Betreuer. Jedenfalls seien die Beklagten im Hinblick auf ihre Gewissensentscheidung entschuldigt, auch habe die Patientin keinen Schaden erlitten.

Das medizinrechtliche Schrifttum kommentierte diese Entscheidungen überwiegend kritisch, weil dadurch die Patientenautonomie ausgehöhlt werde ${ }^{7}$. Noch heftigere Kritik erntete allerdings der 12. Zivilsenat des BGH mit seinem Beschluss vom 17.3.20038. Es war das erste Mal, dass der BGH über den Antrag

\footnotetext{
2 BGHSt 37, 376 (379); 40, 257 (259 ff.)

3 BGHSt 42, 301 (305); 46, 279 (284 f.)

4 Ausführlich Taupitz. (Fn. 1), A 96 ff. m.w.N.; Lipp, FamRZ 2004, 317 ff.; vgl. auch Keilbach, FamRZ 2003, 969 ff.; V ossler, BtPrax 2002, 240 ff.

$5 \quad B V \operatorname{erfG}$ (3. Kammer des 1. Senats) NJW 2002, 206. Der Fall ereignete sich 1992.

$6 \quad$ OLG München NJW-RR 2002, 811 = MedR 2003, 174

7 Ublenbruck, NJW 2003, 1710 (1711 f.); Bender, MedR 2003, 179 f.; Heßler, MedR 2003, 13 ff.;

Rossbruch, PflR 2003, 166 ff.; eher zustimmend dagegen Spickhoff, NJW 2003, 1701 (1709)

8 BGHZ 154, 205 ff. $=$ FamRZ 2003, 748 ff. $=$ BtPrax 2003, 123 ff. $=$ NJW 2003, 1588 ff. $=$ JZ $2003,732 \mathrm{ff}$.
} 
eines Betreuers entscheiden musste, die Einstellung der künstlichen Ernährung eines Komapatienten vormundschaftsgerichtlich zu genehmigen.

Amtsgericht und Landgericht hatten diesen Antrag abgelehnt, weil es dafür im geltenden Recht keine Rechtsgrundlage gebe. Insbesondere könne \1904 BGB nicht analog angewandt werden. Das OLG Schleswig sah es ebenso, legte die Sache aber wegen der anders lautenden Entscheidungen der Oberlandesgerichte Frankfurt ${ }^{9}$ und Karlsruhe ${ }^{10}$ dem BGH vor ${ }^{11}$. Der BGH entschied, dass das Betreunngsrecht ein solches Genehmigungsverfahren in bestimmten Fällen vorsehe, und verwies die Sache zur Durchführung dieses $V$ erfahrens an das Amtsgericht zurück. Bei dieser Gelegenheit äußerte er sich in Form von obiter dicta ${ }^{12}$ u.a. auch zur Zulässigkeit der Einstellung lebenserhaltender Maßnahmen durch den Betreuer sowie zur Verbindlichkeit einer Patientenverfügung. Zur Begründung seines Antrags hatte sich der Betrener nämlich auf die einschlägige Patientenverfügung berufen.

Der Beschluss rief sehr gegensätzliche Reaktionen in der Fachöffentlichkeit hervor. Manche sehen dadurch Rechtssicherheit für Patienten, deren Vertreter und Ärzte geschaffen ${ }^{13}$ und die Rolle des VormG geklärtt ${ }^{14}$. Andere bezeichnen den Beschluss als „Paukenschlag der Konfusion“15, der eine unpraktikable ${ }^{16}$,Justizverwaltung in Schicksalsdingen"17 einführe und die verfassungsrechtlichen Grenzen des Richterrechts überschreite ${ }^{18}$. Ähnlich widersprüchlich sind auch die Einschätzungen, welcher Stellenwert künftig einer Patientenverfügung zukommt ${ }^{19}$.

Vor diesem Hintergrund setzte die Bundesjustizministerin eine Arbeitsgruppe ein und beauftragte sie zu untersuchen, ob zur Sicherung der Patientenautonomie am Lebensende gesetzliche Regelungen erforderlich sind. Der Bericht dieser nach ihrem Vorsitzenden benannten „Kutzer-Kommission“ (im Folgenden: KK) wurde am 10.06.2004 veröffentlicht und war Grundlage für den Referentenentwurf

\footnotetext{
9 OLG Frankfurt FamRZ 1998, 1137; 2002, 575

10 OLG Karlsrube FamRZ 2002, 488

11 OLG Schleswig FamRZ 2003, 554 = NJW-RR 2003, 435

12 Entscheidungsgegenstand war allein die Frage, ob das geltende Recht ein solches Genehmigungsverfahren überhaupt kennt und das VormG daher verpflichtet ist, es auf Antrag des Betreuers durchzuführen, vgl. Lipp, FamRZ 2003, 756; Deutsch, NJW 2003, 1567.

13 Strätling/Sedemund-Adib/Bax/Scharf/Fieber/Schmucker, Entscheidungen am Lebensende in Deutschland, Sonderveröffentlichung zu BtPrax 5/2003, 44, im Internet unter www.bundesanzeiger.de/old/zeitschri/btprax/komm_bgh.pdf (Stand: 30.1.2006)

14 Deutsch, NJW 2003, 1567 f.

15 Höfling/Rixen, JZ 2003, 884 (894)

16 Paebler, BtPrax 2003, $141 \mathrm{ff}$.

17 Hol̨hauer, FamRZ 2003, 991 (992); ähnlich Kutzer, ZRP 2003, 213 ff.: Vormundschaftsrichter als Schicksalsbeamter; kritisch auch Paehler, BtPrax 2003, 141 (143)

18 Hufen, ZRP 2003, 248 ff.; Höfling/Rixen, JZ 2003, 884 (893 f.)

19 Positiv z.B. Borasio/Putz/Eisenmenger, DÄBl. 2003, A 2062 ff.; Perau, RNotZ 2003, 263 (264); kritisch dagegen Bienwald, MDR 2003, 694 (695); Kutzer, ZRP 2003, 213 f.; Rossbruch, PflR 2003, 254 (255); Spickhoff, JZ 2003, 739 (741); Ublenbruck, NJW 2003, 1710; Verrel, NStZ 2003, 449 (451); vgl. zum Ganzen auch Lipp, BtPrax 2004, 18 ff.; Roth, JZ 2004, 494 ff.
} 
Rechtliche Grundlage der Entscheidungsfindung

eines 3. Betreuungsrechtsänderungsgesetzes vom 1.11.2004, der im Bundesjustizministerium konzipiert wurde (im Folgenden: RefE). Parallel dazu erarbeiteten die Bioethik-Kommission des Landes Rheinland-Pfalz (im Folgenden RPf) und die Enquete-Kommission „Ethik und Recht der modernen Medizin“ des Bundestags (im Folgenden: EK) ausführliche Berichte zum Problemkreis der „Sterbehilfe“. Während die „Kutzer-Kommission“ und der Referentenentwurf für eine möglichst weitgehende Anerkennung von Patientenverfügungen eintreten und die Genehmigung durch das VormG für Bevollmächtigte abschaffen und für Betreuer auf Konfliktfälle beschränken wollen, worin die rheinland-pfälzische BioethikKommission außer in der Frage der Genehmigung für Bevollmächtigte übereinstimmt, nimmt die Enquete-Kommission des Bundestags insgesamt eine wesentlich restriktivere Position ein. Die rechtspolitische Debatte ist mittlerweile in vollem Gange ${ }^{20}$.

In dieser Situation erging am 8.6.2005 eine weitere Entscheidung des 12. Zivilsenats des BGH zur Einstellung der künstlichen Ernährung ${ }^{21}$.

Ein Betreuer hatte Klage gegen das Pflegeheim erhoben, weil es sich weigerte, die vom Betreuer in Übereinstimmung mit dem Arzt verlangte Einstellung der künstlichen Ernährung des im Koma liegenden Patienten durchzuführen (,Traunsteiner Fall“). Das OLG München wies die Klage ab 22. Der Heimvertrag verpflichte das Heim zur Lebenserhaltung und nicht zu einer Mitwirkung bei der Sterbehilfe. Auch hätten die Pflegekeäfte und das Heim das Recht, diese Mitwirkung aus Gewissensgründen zu verweigern. Das gelte selbst dann, wenn das Vorm $G$ dies genehmigt habe. Der BGH ließ die Revision $2 u^{23}$. Da der Patient danach verstarb und die Parteien den Rechtsstreit für erledigt erklärten, konnte allerdings kein Urteil in der Sache ergehen. Der BGH hatte gem. I 91 a ZPO nur noch über die Kosten zu entscheiden. In diesem Beschluss äußerte er sich zu zablreichen Fragen der Einstellung einer lebenserhaltenden Bebandlung (,passive Sterbebilfe"), die bereits Gegenstand des Beschlusses vom 17.3.2003 gewesen waren, und präzisierte seine Position ${ }^{24}$.

\footnotetext{
20 Die vorstehend genannten Berichte und Entwürfe und weitere Stellungnahmen sind zusammengestellt auf http://www.aem-online.de/aemaktuell/patientenautonomie.htm (Stand: 30.1.2006); am 8. März 2005 hat die Kammer für öffentliche Verantwortung der Evangelischen Kirche in Deutschland eigene „Überlegungen zum Umgang mit Patientenverfügungen aus evangelischer Sicht“" veröffentlicht, http://www.ekd.de/download/ekd_texte_80.pdf (Stand: 30.1.2006). Ausführlich dazu Lipp, Patientenautonomie (Fn. 1).

21 BGH NJW 2005, 2385 ff.; vgl. dazu Wagenitr, FamRZ 2005, 669 ff.; Lipp, Patientenautonomie (Fn. 1), insbes. S. $53 \mathrm{f}$.

22 OLG München FamRZ 2003, 557; Vorinstanz LG Traunstein NJW-RR 2003, 221

23 BGH, Beschluss v. 24.9.2003 - XII ZR 177/03

24 Vgl. auch Wagenitz, FamRZ 2005, 669 ff.
} 


\section{Grundlegende Fragestellung}

In der Debatte um die „Sterbehilfe“ wird nicht immer sorgfältig genug zwischen der Analyse des geltenden Rechts einerseits und den rechtspolitischen Vorschlägen zur Änderung des geltenden Rechts andererseits unterschieden. Insbesondere das Verfassungsrecht wird gern bemüht, um rechtspolitische Ziele als geltendes (Verfassungs-)Recht darzustellen und ihnen damit die Dignität der Verfassung zu verleihen ${ }^{25}$. Will man sich vergewissern, auf welchen rechtlichen Grundlagen die schwierigen Entscheidungen im konkreten Einzelfall beruhen, muss man sich deshalb zuerst mit den Grundfragen der ärztlichen Behandlung am Ende des menschlichen Lebens befassen ${ }^{26}$ und den gegenwärtigen Stand der Rechtsprechung und Rechtspraxis sorgfältig analysieren.

Die rechtlichen Grundlagen und Grenzen zulässiger „Sterbehilfe“ sind lange Zeit unter dem Gesichtspunkt der Strafbarkeit des Arztes diskutiert worden. Für die ärztliche Behandlung und Versorgung des Patienten am Ende seines Lebens enthält das Strafrecht jedoch nur punktuelle Vorgaben. „Sterbehilfe“ ist daher im Kern eine Frage des Zivilrechts und der - auch grundrechtlich geschützten - Patientenautonomie ${ }^{27}$.

Die ärztliche Behandlung am Ende des menschlichen Lebens ist umso problematischer geworden, je mehr der medizinische Fortschritt die Möglichkeit eröffnet hat, das Leben des Patienten zu erhalten und zu verlängern. Daraus erwächst zugleich die Notwendigkeit zu entscheiden, ob und inwieweit die Errungenschaften der modernen Medizin für die Behandlung des Patienten im konkreten Fall genutzt werden sollen. Die Rechtsordnung muss deshalb festlegen,

1. wer diese Entscheidung treffen kann ${ }^{28}$;

2. nach welchem Maßstab sie getroffen werden muss ${ }^{29}$ und

3. wie diese Entscheidung ggf. um- und durchgesetzt werden kann.

\footnotetext{
25 Vgl. die Berufung auf den - angeblichen - Grundsatz ,in dubio pro vita“ bei EK (Fn. 20), S. 37; Storr, MedR 2002, 436 (441), oder die Abwägung zwischen Lebensschutz und Selbstbestimmungsrecht bei EK (Fn. 20), S. 38 ff.; Duttge (Fn. 1), S. 7, 9; Stackmann, MedR 2003, 490 (491 f.); dagegen zutreffend Hufen, NJW 2001, 849 (851 ff.); RPf(Fn. 20), S. 9, 27 ff.

26 Ebenso Taupitz (Fn. 1), A 10 ff.; Spickhoff, NJW 2000, 2297 ff.; Berger, JZ 2000, 797 ff.

27 Vgl. die Nachweise oben Fn. 1; vgl. auch RPf(Fn. 20), S. 27 ff.

28 Diese Frage ist angesichts der auch grundrechtlich verbürgten Patientenautonomie vorrangig, vgl. Lipp, DRiZ 2000, 231; Taupitz (Fn. 1), A 11; Wagenitz, FamRZ 2005, 669 (672).

29 Darauf konzentriert sich die strafrechtliche Diskussion, weil für die Strafbarkeit des Arztes die Rechtfertigung des Behandlungsabbruchs aufgrund des Willens des Patienten ausschlaggebend sei, vgl. Otto, ZfL 2002, 42 ff.; Verrel, NStZ 2003, 449 (452 f.).
} 


\section{3. Ärztliche Behandlung am Lebensende: Hilfe im Sterben und Hilfe zum Sterben}

\subsection{Rechtliche Struktur der ärztlichen Behandlung}

Für die Versorgung und Behandlung eines Menschen am Ende seines Lebens gilt dasselbe wie für jede andere ärztliche Behandlung. Praktisch gesehen kommen Arzt und Patient nach Untersuchung und Diagnose gemeinsam zur Entscheidung, eine bestimmte Behandlung durchzuführen oder zu unterlassen ${ }^{30}$. Rechtlich spiegelt sich die dialogische Struktur des Behandlungsprozesses in der Verpflichtung des Arztes wider, den Patienten an diesem Prozess kontinuierlich zu beteiligen und über die Bedeutung und Tragweite der vorgeschlagenen Schritte zu informieren ${ }^{31}$. Aus der fachlichen Kompetenz des Arztes folgt die rechtliche Abgrenzung der Verantwortungsbereiche: Der Arzt trägt die Verantwortung für die fachgerechte Untersuchung, Diagnose und Indikation für oder gegen eine bestimmte Behandlung und hat den Patienten hierüber jeweils aufzuklären. Eine vorherige Aufklärung kann zweierlei erreichen: Zum einen kann sie, wie jede ärztliche Aufklärung, den Patienten über seine Krankheit und die möglichen Behandlungsalternativen informieren und ihn so in die Lage versetzen, sich zu entscheiden. Zum anderen kann sie dem später behandelnden Arzt das Verständnis einer Patientenverfügung erleichtern. Unter dem erstgenannten Gesichtspunkt dient die Aufklärung der Wahrnehmung des Selbstbestimmungsrechts des Patienten; auf sie kann der Patient deshalb auch verzichten ${ }^{32}$. Der Patient entscheidet dann eigenständig, ob er in eine bestimmte ärztliche Maßnahme, sei es zur Untersuchung oder zur Behandlung, einwilligt oder ob er diese ablehnt ${ }^{33}$.

Daraus folgt, dass der Arzt kein eigenständiges Behandlungsrecht hat. Recht und Pflicht zur Behandlung ergeben sich allein aus dem Auftrag des Patienten. Jede ärztliche Maßnahme bedarf darüber hinaus der gesonderten Einwilligung des Patienten, weil damit das Selbstbestimmungsrecht des Patienten über seine Person betroffen ist. Diese Einwilligung muss vor Beginn der Maßnahme eingeholt werden und setzt eine entsprechende Aufklärung durch den Arzt voraus ${ }^{34}$. Widerruft der Patient seine Zustimmung, wird die weitere Behandlung unzulässig 35 .

\footnotetext{
30 Burchardi, Festschrift H.-L. Schreiber, 2003, S. 615 (617); Borasio/Putz/Eisenmenger, DÄBl. 2003, A 2062 (2064); Wagenitr, FamRZ 2005, 669 (670)

31 Deutsch/Spickboff, Medizinrecht, 5. Aufl. 2003, Rn. 16, S. 187; Ublenbruck/Laufs, in:

Laufs/Uhlenbruck, Handbuch des Arztrechts, 3. Aufl. 2002, §52 Rn. 9; Taupitz. (Fn. 1), A 12 ff.;

Wagenits, FamRZ 2005, 669 (670); Lipp, FamRZ 2004, 317 (318); RefE (Fn. 20), S. 7

32 Vgl. nur Taupitz (Fn. 1), A 28 ff.

33 Vgl. nur die Nachweise in Fn. 31

34 BGHZ 29, 46 (49 ff.); BGH NJW 1980, 1333; BGH NJW 1993, 2372 (2373 f.); vgl. dazu ausführlich Voll, Die Einwilligung im Arztrecht, 1996, S. 7 ff.; Wagenits, FamRZ 2005, 669 (671); Laufs, in: Laufs/Uhlenbruck (Fn. 31), \ 61 Rn. 14 f., \ 63

35 Vgl. hierzu BGH NJW 2005, 2385 (2386); Wagenitr, FamRZ 2005, 669 (671)
} 
Die rechtliche Grundstruktur der ärztlichen Behandlung missachten all diejenigen, die fragen, ob der Abbruch der Behandlung zulässig ist ${ }^{36}$. Sie verkehren damit die Legitimationslast für eine ärztliche Behandlung in ihr Gegenteil. Denn nicht der Verzicht, sondern die Aufnahme der Behandlung, nicht ihr Abbruch, sondern ihre weitere Durchführung bedürfen der Einwilligung des Patienten ${ }^{37}$. Auch eine lebensverlängernde Maßnahme ist nur zulässig, wenn und solange ihr der Patient zustimmt. Lehnt der Patient die Behandlung ab, müssen Arzt, Pflegepersonal und Klinik bzw. Pflegeheim dem Folge leisten ${ }^{38}$. Dabei kommt es nicht darauf an, ob sich die Entscheidung des Patienten in den Augen des Arztes (oder eines anderen) als vernünftig oder unvernünftig darstellt ${ }^{39}$. Behandelt er den Patienten gegen dessen Willen, begeht er eine Körperverletzung.

Die Ablehnung der Behandlung ist dabei jederzeit möglich, kann also auch nach Beginn der Behandlung erfolgen. Ein vertraglicher Verzicht auf den Widerruf einer einmal erteilten Einwilligung ist wegen ihres Persönlichkeitsbezugs ausgeschlossen $^{40}$. Das verkennt das OLG München im Traunsteiner Fall, wenn es den vom Patienten bzw. Betreuer gewünschten Abbruch der künstlichen Ernährung wegen des Heimvertrages für unbeachtlich hält ${ }^{41}$. Zu Recht hat demgegenüber der BGH in seinem Beschluss vom 8.6.2005 ausgeführt, dass das Recht des Patienten zur Bestimmung über den eigenen Körper einem antizipierten Verzicht (hier: in einem Heimvertrag) nicht zugänglich sei ${ }^{42}$. Vielmehr bedarf jede neue ärztliche Maßnahme stets seiner Einwilligung.

Allerdings verleiht die Patientenautonomie dem Patienten nur ein Abwehrrecht gegen eine vom Arzt vorgeschlagene Behandlung, verschafft ihm aber keinen Anspruch auf eine bestimmte Behandlung. Es ist keine Frage der Patientenautonomie, sondern der allgemeinen Rahmenbedingungen der Behandlung, ob z.B. eine bestimmte Behandlungsmethode von der Krankenkasse finanziert wird oder ob sie in dem Krankenhaus, in dem der Patient liegt, überhaupt verfügbar ist ${ }^{43}$. Vor allem kann der Arzt eine an sich mögliche Behandlung verweigern, für die

\footnotetext{
36 Vgl. z.B. EK (Fn. 20), 37; Duttge (Fn. 1), S. 9, 53

37 BGHZ 154, 205 (210 f., 212); BGHSt 37, 376 (378); BGH NJW 2005, 2385 (2386); Lipp, BtPrax 2002, 47, und FamRZ 2004, 317 (318); Wagenitz, FamRZ 2005, 669 (671); Hufen, NJW 2001, 849 (850); Taupitz (Fn. 1), A 18, 44; RPf(Fn. 20), S. 28; unzutreffend daher der Ansatz bei Stackmann, MedR 2003, 490 (492); Storr, MedR 2002, 436 (438), die zwischen Lebensschutz und Selbstbestimmungsrecht abwägen.

38 Zutreffend BGH NJW 2005, 2385 (2386)

39 RGSt 25, 375 (378 f.); BGHSt 11, 111 (114); BGH NJW 1980, 1333 (1334); BGH NJW 1980, 2751 (2753); BGHZ 90, 103 (105 f.); BGH NJW 2005, 2385 (2386); Lipp, Freiheit und Fürsorge: Der Mensch als Rechtsperson, 2000, S. $60 \mathrm{ff}$.

40 Kobte, AcP 185 (1985), 105 (137 f.); Deutsch/Spickhoff(Fn. 31), Rn. 197; Ublenbruck/Kern, in: Laufs/Uhlenbruck (Fn. 31), J 71 Rn. 1, \ 81 Rn. 7

41 OLG München FamRZ 2003, 557 (558)

42 BGH NJW 2005, 2385 (2385)

43 Zur Verteilungsproblematik vgl. Spickhoff, NJW 2000, 2297 (2298); Taupitz. (Fn. 1), A 25 ff.
} 
Rechtliche Grundlage der Entscheidungsfindung

keine medizinische Indikation besteht ${ }^{44}$. Ist eine Maßnahme gar kontraindiziert, darf er sie unter keinen Umständen durchführen, und zwar auch nicht auf ausdrücklichen Wunsch des Patienten ${ }^{45}$.

An diesen Zusammenhang von tatsächlicher Verfügbarkeit einer bestimmten Maßnahme, ihrer medizinischen Indikation und der Einwilligung des Patienten knüpft der BGH an, wenn er ausführt, dass für eine Einwilligung (im Fall des BGH: des Betreuers als gesetzlichem Vertreter des Patienten) erst dann Raum sei, wenn der Arzt eine Behandlung ,anbiete"46. Damit wird deutlich, dass die Konkretisierung des medizinischen Korridors ${ }^{47}$ bzw. das Erarbeiten einer medizinischen Indikation im konkreten Fall ${ }^{48}$ zwar im Gespräch mit dem Patienten erfolgt ${ }^{49}$, aber letztlich allein in den Verantwortungsbereich des Arztes fällt ${ }^{50}$.

\section{2 „Hilfe im Sterben“ und „Hilfe zum Sterben“}

Liegt ein Patient im Sterben, hat also der Sterbeprozess bereits begonnen, ist eine lebensverlängernde Behandlung (z.B. mit den Mitteln der Intensivmedizin) nicht mehr indiziert ${ }^{51}$. Ihr Unterlassen bedeutet keine Tötung des Patienten durch den Arzt. Geboten ist vielmehr ärztliche Hilfe und Begleitung im Sterbeprozess, die so genannte „Hilfe im Sterben“52. Diese Änderung des Behandlungsziels muss zwar nach allgemeinen Grundsätzen zwischen Arzt und Patient besprochen werden. Die Entscheidung gegen lebenserhaltende Maßnahmen beruht jedoch auf der vom Arzt festgestellten und verantworteten fehlenden medizinischen Indikation, nicht auf einem Widerspruch des Patienten gegen diese Maßnahmen ${ }^{53}$.

Hält der Arzt dagegen eine Maßnahme aus medizinischer Sicht für indiziert, obliegt es dem Patienten zu bestimmen, ob, wie lange und in welcher Weise er behan-

44 Laufs, NJW 1998, 3399 (3400); Schöne-Seiffert, Referat auf dem 63. DJT, 2000, K 53, 57; Spickhoff, NJW 2000, 2297 (2298); Taupitz. (Fn. 1), A 23 f.; Heyers, Passive Sterbehilfe bei entscheidungsunfähigen Patienten und das Betreuungsrecht, 2001, S. 29 ff.

45 OLG Karlsrube MedR 2003, 104 ff.; OLG Düsseldorf VersR 2002, 611; OLG Köln VersR 2000, 492; Deutsch/Spickhoff(Fn. 31), Rn. 12

46 BGHZ 154, 205 (225)

47 Taupitz.(Fn. 1), A 24

48 Spickhoff, NJW 2003, 1701 (1709)

49 Borasio/Putz/Eisenmenger, DÄBl 2003, A 2062 (2064)

50 Ankermann, MedR 1999, 387 (389); Dodegge/Fritsche, NJ 2001, 176

51 Vgl. die Grundsätze der Bundesärztekammer zur ärztlichen Sterbebegleitung vom 30.4.2004, Ziff. I., DÄBl 2004, A 1298; Schreiber, Festschrift Deutsch, 1999, S. 773 ff.; zu dieser „objektiven“ Grenze der Behandlungspflicht auch Opderbecke/Weißauer, MedR 1998, 395 (397); Saliger, KritV 2001, $382 \mathrm{ff}$.

52 BGHSt 40, 257 (260); zur rechtspolitischen Diskussion über die „Sterbehilfe“ in den verschiedenen Phasen: Lipp, Patientenautonomie (Fn. 1), S. 16 ff.

53 Ankermann, MedR 1999, 387 (389); Lipp, in: May/Geißendörfer/Simon/Strätling, Passive Sterbehilfe: besteht gesetzlicher Regelungsbedarf?, 2002, S. 37 (52 f.); MünchKomm/Schwab, BGB, 4. Aufl. 2002, \1904 BGB Rn. 38. Die Bedeutung der medizinischen Indikation übersieht die Kritik von Duttge (Fn. 1), S. 50. Da es hierbei um generelle Aussagen über den medizinischen Wert einer Behandlung geht, liegt darin keine unzulässige Bewertung des individuellen Lebens der Patienten. 
delt und versorgt werden will. Lehnt der Patient eine angebotene lebenserhaltende Maßnahme ab, oder widerruft er seine früher erteilte Einwilligung, darf der Arzt diese Maßnahme nicht durchführen ${ }^{54}$. Stirbt deshalb der Patient, liegt darin keine Tötung durch den Arzt, weil der Arzt gar nicht mehr behandeln darf. Es ist also kein Fall der „aktiven Sterbehilfe“, d.h. der Tötung auf Verlangen (\$216 StGB). Vielmehr handelt es sich um die „Hilfe zum Sterben“ genannte Einstellung der Behandlung auf Wunsch des Patienten. Die rechtliche Zulässigkeit der „Hilfe zum Sterben" ist also nichts anderes als die Kehrseite des Selbstbestimmungsrechts des Patienten 5 .

Die Unterscheidung zwischen „passiver Sterbehilfe“ als „Hilfe beim Sterben“ in der unmittelbaren Sterbephase und der „Hilfe zum Sterben“ in allen anderen Fällen ${ }^{56}$ verweist demnach auf die unterschiedlichen Gründe für die Einstellung der lebenserhaltenden Maßnahmen: Im Sterbeprozess ist sie zulässig, weil es an einer medizinischen Indikation fehlt, hat der Sterbevorgang noch nicht eingesetzt, darf eine indizierte lebenserhaltende Maßnahme nur mit Willen des Patienten unterlassen oder abgebrochen werden ${ }^{57}$.

Der 1. Strafsenat des BGH hat darauf bereits 1994 in dem so genannten Kemptener Fall hingewiesen. Er hat dort die Sterbephase, in der der Arzt auf lebensverlängernde Maßnahmen verzichten darf (zu ergänzen: weil sie nicht mehr indiziert sind), in Anlehnung an die damaligen Richtlinien der Bundesärztekammer zur Sterbehilfe dahingehend umschrieben, dass das Grundleiden des Patienten irreversibel ist, einen tödlichen Verlauf genommen hat und der Tod in kurzer Zeit eintreten wird ${ }^{58}$. Damit sollte die Sterbephase von den Situationen abgegrenzt werden, in denen ein Abbruch der lebenserhaltenden Behandlung nur mit Willen des Patienten zulässig ist („Hilfe zum Sterben“). Dieses Abgrenzungskriterium hat der 12. Zivilsenat des BGH in seinem Beschluss vom 17.3.2003 als objektive strafrechtliche Grenze für die Zulässigkeit der Sterbehilfe missverstanden: Lägen diese Voraussetzungen nicht vor, sei die Einstellung der lebenserhaltenden Maßnahme rechtswidrig ${ }^{59}$. Eine solche Grenze existiert jedoch nicht; sie wurde vom 1. Strafsenat auch nicht aufgestellt ${ }^{60}$. Sie wäre im Übrigen ein verfassungswidriger Eingriff

\footnotetext{
54 Vgl. hierzu zutreffend BGH NJW 2005, 2385 (2386); vgl. auch Wagenitz, FamRZ 2005, 669 (671)

55 Zum Vorstehenden ausführlich Lipp, in: Passive Sterbehilfe (Fn. 53), S. 41 f.; Hufen, NJW 2001, 849 (851); ebenso RPf(Fn. 20), S. 31

56 Vgl. BGHSt 40, 257 (260) und ihm folgend das OLG Frankfurt NJW 1998, 2747 (2748)

57 Vgl. zur rechtspolitischen Diskussion über die „Sterbehilfe“: Lipp, Patientenautonomie (Fn. 1), S. 16 ff.

58 BGHSt 40, 257 (260), unter Hinweis auf die (damaligen) Richtlinien der Bundesärztekammer für die Sterbehilfe, Ziff. II.d., MedR 1985, 38. Die heutigen Richtlinien (Fn. 51), Ziff. I, beschreiben sie als ,irreversibles Versagen einer oder mehrerer vitaler Funktionen, bei denen der Eintritt des Todes in kurzer Zeit zu erwarten ist".

59 BGHZ 154, 205 (214 ff.)

60 Vgl. BGHSt 40, 257 (260 f.); Kutzer, ZRP 2003, 213 f.; ebenfalls kritisch gegenüber dieser Einschränkung, wenngleich ohne zu erwähnen, dass BGHSt 40, 257 ff. die „Hilfe zum Sterben“ ausdrücklich für zulässig erklärt hat, auch Höfling/Rixen, JZ 2003, 884 (885ff.); Spickhoff, JZ 2003, 739
} 
in das Selbstbestimmungsrecht des Patienten, weil er dann gegen seinen Willen zwangsweise behandelt würde ${ }^{61}$. Der Patient hat das Recht, jede lebenserhaltende Maßnahme abzulehnen. Die Aussage des 12. Zivilsenats in diesem Beschluss, „Sterbehilfe“ sei nur im Falle eines ,irreversibel tödlich verlaufenden Grundleidens" zulässig, ist daher unzutreffend ${ }^{62}$. Als obiter dictum ${ }^{63}$ bindet es freilich die Rechtspraxis nicht. Es braucht deshalb nicht relativiert ${ }^{64}$, sondern sollte bei nächster Gelegenheit vom Senat selbst aufgegeben werden ${ }^{65}$.

Der 12. Zivilsenat des BGH hat in seinem Beschluss vom 8.6.2005 einen ersten Schritt in diese Richtung unternommen. Dort heißt es, dass die strafrechtlichen Grenzen einer Sterbehilfe im weiteren Sinn („Hilfe zum Sterben“) bislang „,nicht hinreichend geklärt" erscheinen ${ }^{66}$. Damit gibt der 12. Zivilsenat zwar seine Ansicht über die strafrechtliche Grenze der „Sterbehilfe“ auf, die er im Jahre 2003 aus der Entscheidung des 1. Strafsenats im Kemptener Fall zu Unrecht herausgelesen hat. Allerdings fehlt derzeit eine ausdrückliche höchstrichterliche Feststellung, dass eine solche Grenze nicht existiert; die wünschenswerte Klarheit ist damit noch nicht erreicht ${ }^{67}$.

\section{Der einwilligungsunfähige Patient}

Problematisch wird die Beteiligung des Patienten am Behandlungsprozess, wenn er einwilligungsunfähig ist. Der Arzt kann dann weder die Diagnose mit dem Patienten besprechen, noch ihn über die Möglichkeiten einer Behandlung aufklären. Der Patient selbst fällt dann als Partner des Arztes im Behandlungsprozess aus. Vor allem aber kann der Patient seine Entscheidung über Ob, Wie und Dauer der Behandlung nicht mehr selbst treffen. Die fehlende tatsächliche Fähigkeit zur Selbstbestimmung lässt allerdings das Recht des Patienten zur Selbstbestimmung

(740 f.); Strätling/Sedemund-Adib/Bax/Scharf/Fieber/Schmucker (Fn. 13), 16 ff.; Verrel, NStZ 2003, 449 (451); anders dagegen EK (Fn. 20), S. 20 („Ausnahmefall“); Duttge (Fn. 1), S. 45 ff., 52 f.

61 Hufen, ZRP 2003, 248 (252)

62 Kutzer, ZRP 2003, 213 f.; Lipp, FamRZ 2003, 756

63 Dazu oben Fn. 12

64 Vgl. die Versuche einer „,weiten“ Interpretation bei Bübler/Stoly, FamRZ 2003, 1622 f.; oder die Beschränkung auf Fälle, in denen kein Wille des Patienten feststellbar ist, bei Habne, FamRZ 2003, 1619 (1620 f.).

65 Z.B. indem man den „,irreversibel tödlichen Verlauf des Grundleidens“ dahingehend versteht, dass die Krankheit ohne Behandlung zum Tod führt (so Habne im Interview in der FAZ v.

18.7.2003). Diese Voraussetzung ist nämlich stets erfüllt, wenn es um die Ablehnung einer lebenserbaltenden ärztlichen Maßnahme und damit um Sterbehilfe geht (Taupits, Protokoll des Nationalen Ethikrats v. 11.6.2003, S. 17, abrufbar unter http://www.ethikrat.org/texte/pdf/Forum_Patient_2003-0611_Protokoll.pdf (Stand: 30.1.2006)).

66 BGH NJW 2005, 2385 (2386)

67 Vgl. zur rechtspolitischen Diskussion: Lipp, Patientenautonomie (Fn. 1), S. 16 ff. 
nicht entfallen ${ }^{68}$. Das Recht des Patienten zur Teilnahme am Behandlungsprozess bleibt daher ebenso bestehen wie sein Recht, einer vorgeschlagenen Untersuchungs- oder Behandlungsmaßnahme zuzustimmen oder sie abzulehnen. Der Patient kann dieses Recht jedoch nicht mehr selbständig ausüben.

In dieser Situation sind zwei Möglichkeiten denkbar: Entweder hat der Patient selbst schon zuvor, d.h. bevor er einwilligungsunfähig wurde, erklärt, ob er den nunmehr vom Arzt vorgeschlagenen Maßnahmen zustimmt, oder der Patient hat sich dazu nicht geäußert. Im zweiten Fall muss ein anderer an Stelle des Patienten die nötigen Entscheidungen treffen. Hierzu ist in erster Linie die vom Patienten bestimmte Vertrauensperson berufen, sei es der Arzt ${ }^{69}$ oder ein Bevollmächtigter, andernfalls der Betreuer als gesetzlicher Vertreter ${ }^{70}$. Erst wenn in einem Notfall noch nicht einmal eine Eilentscheidung des an Stelle eines Betreuer tretenden VormG nach \1846 BGB herbeigeführt werden kann, darf und muss der Arzt, zusätəlich zu seiner Aufgabe als behandelnder Arzt und ohne vom Patienten dazu berufen zu sein, auch die Rolle des Wahrers des Patienteninteresses übernehmen $^{71}$. Sein Handeln ist dann nach den Grundsätzen der Geschäftsführung ohne Auftrag ( $\iint 677$ ff. BGB) bzw., soweit es um den Eingriff in die körperliche Integrität geht, aufgrund einer mutmaßlichen Einwilligung des Patienten gerechtfer$\operatorname{tigt}^{72}$.

\section{Die Patientenverfügung}

Für die stellvertretende Erklärung eines Bevollmächtigten oder Betreuers, oder für eine Notfallentscheidung des Arztes ist aber nur Raum, wenn der Patient selbst nichts erklärt hat. Die vorrangige Frage ist daher, inwieweit jemand schon im Vorfeld das $\mathrm{Ob}$ und Wie seiner späteren Behandlung festlegen kann. Hierüber wird derzeit rechtspolitisch heftig debattiert ${ }^{73}$. Im Folgenden geht es dagegen um das geltende Recht.

\subsection{Erscheinungsformen der Patientenverfügung}

Vorausverfügungen werden allgemein akzeptiert, wenn sie vom Patienten im Gespräch mit dem ihn später behandelnden Arzt erklärt werden, also beispielsweise

\footnotetext{
68 Lipp, DRiZ 2000, 231 (233 f.); Höfling, JuS 2000, 111 (113 f.); Hufen, NJW 2001, 849 (850 ff.); vgl. auch 12. Zivilsenat BGH im Traunsteiner Fall: BGH NJW 2005, 2385 (2386)

69 $K K$ (Fn. 20), S. 15. Diese Möglichkeit wird häufig übersehen.

70 \1896 II 2 BGB legt den Vorrang des Bevollmächtigten fest.

71 Zum Vorrang der Vertreterbestellung BGHZ 29, 46 (52); BGH NJW 1966, 1855 (1856); Lipp, BtPrax 2002, 47 (51) m.w.N.

72 Deutsch/Spickhoff(Fn. 31), Rn. 83

73 Siehe oben Abschnitt 1. Vgl. im Übrigen zur rechtspolitischen Diskussion: Lipp, Patientenautonomie (Fn. 1), S. 25 ff.
} 
im Vorfeld einer unter Narkose erfolgenden Behandlung ${ }^{74}$. Als „Patientenverfügung“ bezeichnet man eine Vorauserklärung typischerweise jedoch erst dann, wenn sie unabhängig von einer konkreten Behandlungssituation erfolgt. Sie richtet sich deshalb meist auch nicht an einen bestimmten einzelnen Arzt, den der Patient bereits kennt, sondern ganz generell an alle Ärzte, Pflegekräfte usw., die den Patienten künftig einmal behandeln werden. Die Patientenverfügung ist, mit anderen Worten, typischerweise eine allgemeine Erklärung, die an einen noch unbestimmten Adressaten gerichtet ist. Wer sie abgibt, will dem jeweiligen Aržt gegenüber erklären, er stimme bestimmten Maßnahmen zu bzw. wesentlich häufiger, er lehne sie $\mathrm{ab}^{75}$.

Allerdings stellt die eben skizzierte Form nur eine unter vielen Erscheinungsformen „der“ Patientenverfügung dar. Neben derartigen antizipierten Erklärungen werden auch Dokumente als Patientenverfügungen bezeichnet, in denen jemand seine Wünsche, Einstellungen und Werthaltungen darlegt, die dann in der konkreten Behandlungssituation erst konkretisiert werden müssen ${ }^{76}$.

\subsection{Rechtliche Verbindlichkeit der verschiedenen Formen von Patien- tenverfügungen}

Legt jemand in einer Patientenverfügung seine Wünsche, Einstellungen und Werthaltungen dar, die dann in der konkreten Behandlungssituation erst konkretisiert werden müssen, enthält sie keine verbindliche Erklärung des Patienten für seine spätere Behandlung, sondern Mitteilungen über seine Wünsche und Vorstellungen, die als Indizien darüber Auskunft geben, wie er in der aktuellen Situation entschieden hätte (mutmaßlicher Wille) ${ }^{77}$. Diese Mitteilung von Wünschen, Wertvorstellungen o.ä. erzeugt keine unmittelbare Rechtswirkung. In dieser Situation muss daher erst eine andere Person, d.h. ein Vertreter, der Arzt oder ein Angehöriger, den mutmaßlichen Willen des Patienten feststellen und umsetzen ${ }^{78}$. Eine solche „,narrative“ Patientenverfügung ist von vorneherein als Mitteilung des Patienten über sich selbst und seine Vorstellungen gedacht und als solche zu beachten, d.h. als Information und Indiz für andere (Vertreter, Arzt usw.) bei der Ermittlung des mutmaßlichen Willens des Patienten. Für den Betreuer legen das SS 1901 III 1

74 Deutsch/Spickhoff(Fn. 31), Rn. 199; Wagenity, FamRZ 2005, 669 (671)

75 Taupitz.(Fn. 1), A 105 f. m.w.N.; Schwab, Festschrift Henrich, 2000, S. 511 (516 f., 529 f.)

76 Vgl. z.B. Sass/Kielstein, Patientenverfügung und Betreuungsvollmacht, 2001, S. 50 ff., 58; Wagenitr, FamRZ 2005, 669 (671)

77 Viele verstehen ,die“ Patientenverfügung generell als ein solches Indiz, vgl. z.B. Spann, MedR 1983, 13 ff.; Laufs, NJW 1997, 1609 (1616); ders., NJW 1998, 3399 (3400); Spick.hoff, NJW 2000, 2297 (2301 f.); Deutsch/Spickhoff(Fn. 31), Rn. 513 ff.; Roxin, Strafrecht Allgemeiner Teil, 3. Aufl. 1997, J 13 Rn. 66

78 Vgl. zu den „Schwachpunkten“ bei der Ermittlung des mutmaßlichen Willens Wagenitz, FamRZ 2005, 669 (672) 
und 2, 1901a BGB bzw. \1901 II BGB ausdrücklich fest, und zwar unabhängig von der Einhaltung bestimmter Wirksamkeitsvoraussetzungen ${ }^{79}$.

Zunehmend hat sich jedoch die Einsicht durchgesetzt, dass eine Patientenverfügung auch mehr enthalten kann als bloße Indizien für den mutmaßlichen Willen des Patienten: Sie kann eine eigene Erklärung des Patienten enthalten, mit der er sein Selbstbestimmungsrecht im Hinblick auf eine künftige Behandlung ausübt ${ }^{80}$. Dieser Ansicht hat sich mit dem obiter dictum des 12. Zivilsenats ${ }^{81}$ nun auch der $\mathrm{BGH}$ angeschlossen. Eine derartige Patientenverfügung stellt eine vorweggenommene Einwilligung bzw. Ablehnung des Patienten dar, für die dieselben Grundsätze gelten wie für jede andere Einwilligung. Sie ist damit eine an den Arzt gerichtete Erklärung, die keiner bestimmten Form bedarf und auch ohne Aufklärung durch den Arzt wirksam ist, weil der Patient darauf verzichtet hat ${ }^{82}$. Sie bindet zwar den Arzt, nicht aber den Patienten selbst, kann also jederzeit widerrufen oder geändert werden. Eine derartige Patientenverfügung gilt demnach solange, bis der Patient seine Erklärung selbst ändert ${ }^{83}$. Sie darf daher nicht mit dem spekulativen Argument unterlaufen werden, dass der Patient vielleicht etwas anderes gewollt haben könnte ${ }^{84}$. Ihre rechtliche Verbindlichkeit endet vielmehr erst dann, wenn konkrete Anhaltspunkte für eine Willensänderung des Patienten vorliegen ${ }^{85}$.

Diese Grundsätze für eine antizipierte Einwilligung bzw. Ablehnung ergeben sich aus den allgemein anerkannten Regeln, die für die Einwilligung in eine ärztliche Maßnahme und für deren Ablehnung gelten. Sie gelten deshalb unabhängig davon, wie man die umstrittene Frage nach der Rechtsnatur der Einwilligung beantwortet ${ }^{86}$.

79 Palandt/Diederichsen, BGB, 63. Aufl. 2004, \1901 BGB Rn. 5

80 So z.B. die Richtlinien der Bundesärztekammer (Fn. 51), Ziff. IV. und V.; vgl. schon die Handreichungen der BÄK für Ärzte zum Umgang mit Patientenverfügungen, DÄBl. 1999, A 2720, Ziff. 1.1.; ebenso RPf(Fn. 20), S. 35 ff.; Taupitz. (Fn. 1), A 105 ff.; Lipp, DRiZ 2000, 231 (234); Palandt/Diederichsen (Fn. 79), vor $\$ 1896$ BGB Rn. 9; Eisenbart, Patienten-Testament und Stellvertretung in Gesundheitsangelegenheiten, 2. Aufl. 2000, S. 47 ff.; Röver, Einflußmöglichkeiten des Patienten im Vorfeld medizinischer Behandlung, 1997, S. 79 ff.; Schaffer, BtPrax 2003, 143 (146 f.); aus medizinethischer Sicht May, Autonomie und Fremdbestimmung, 2. Aufl. 2001, S. 185 ff.; im Ergebnis ähnlich, wenngleich weiterhin auf den mutmaßlichen Willen abstellend, Schreiber, Festschrift Deutsch, 1999, S. 773 (782)

81 BGHZ 154, 205 (210 f.); zum Charakter der Aussage als obiter dictum oben Fn. 12

82 Vgl. Taupitz.(Fn. 1), A 28 ff.; Wagenitr, FamRZ 2005, 669 (671)

83 Dazu Lipp, in: Passive Sterbehilfe (Fn. 53), S. 43 f.; ebenso die Handreichungen der BÄK für Ärzte zum Umgang mit Patientenverfügungen, DÄBl. 1999, A 2720, Ziff. 6

84 Taupitz (Fn. 1), A 41, S. 106 ff.; Wagenitz, FamRZ 2005, 669 (671)

85 Ebenso die Richtlinien der Bundesärztekammer (Fn. 51), Ziff. IV.; Eisenbart (Fn. 80), S. 67; Baumann/Hartmann, DNotZ 2000, 594 (608 ff.); KK (Fn. 20), S. 17 (etwas anders allerdings S. 8, zu Recht kritisch deshalb an dieser Stelle Müller-Busch in Fn. 1); anders z.B. Taupitz (Fn. 1), A 115 86 Roth, JZ 2004, 494 (497 f. mit Fn. 53); Erman/Holz̧̧auer, BGB, 11. Aufl. 2004, \1901a BGB Rn. 6 f.; unzutreffend deshalb der Ansatz von Duttge (Fn. 1), S. 14 ff. 


\subsection{Notwendigkeit der Auslegung}

Übt der Patient sein Selbstbestimmungsrecht aus und erklärt im Voraus sein Einverständnis oder seine Ablehnung, ist das zugleich mit der Last der Selbstverantwortung verbunden ${ }^{87}$. Wie bei jeder anderen Einwilligung in eine Behandlung oder bei deren Ablehnung trägt der Patient auch bei einer Erklärung in Gestalt einer Patientenverfügung das Risiko, dass er sie nicht mehr rechtzeitig ändern $k^{k}{ }^{8} n^{88}$. Dieses Risiko beruht weder auf einer Selbstbindung des Patienten, noch auf dem Verkehrsschutz zugunsten des Arztes oder anderer Adressaten ${ }^{89}$. Die Rechtsordnung kennt bei der Einwilligung weder das eine noch das andere ${ }^{90}$. Die Änderungslast ist vielmehr Folge des Umstands, dass ein einmal erklärter Wille des Patienten Geltung und Beachtung verlangt, bis der Patient selbst seinen Willen für andere erkennbar ändert ${ }^{91}$ bzw., falls er dazu nicht mehr in der Lage ist, konkrete Anhaltspunkte dafür bestehen, dass er seinen Willen geändert hätte ${ }^{92}$.

Bevor man danach fragt, ob die Patientenverfügung die anstehende Behandlungssituation erfasst oder ob der Patient seinen erklärten Willen geändert hat, ist daher stets zu prüfen, ob er dieses Risiko überhaupt eingehen und eine an Arzt und Pflegepersonal gerichtete verbindliche Erklärung (z.B. durch Gebrauch eines Formulars für eine Patientenverfügung ${ }^{93}$ ) abgeben oder ob er (z.B. im Gespräch mit Angehörigen oder Freunden) lediglich seine Wünsche und Ansichten mitteilen wollte 94 . Wollte er jedoch eine verbindliche Anweisung erteilen, ist sie für den vorgesehenen Fall rechtlich bindend.

Jede Patientenverfügung bedarf daher der Auslegung, d.h. der Feststellung ihres Inhalts dahingehend, ob der Patient eine verbindliche Erklärung abgeben wollte, für welche Fälle sie gedacht ist und welche Anweisungen der Patient hierfür gegeben hat ${ }^{95}$. Diese Auslegung ist Aufgabe derjenigen, an die sich eine Patientenverfügung richtet, d.h. der an einer Behandlung beteiligten Ärzte und Pflegekräfte, aber auch des Vertreters des Patienten oder seiner Angehörigen ${ }^{96}$. Sie dürfen sich dabei nicht auf den Text des Dokuments oder auf die mündliche Äußerung als

87 Taupitz (Fn. 1), A 13

88 Darauf weist hin Diederichsen, Festschrift Schreiber (Fn. 30), S. 635 (648); vgl. auch Wagenits, FamRZ 2005, 669 (671)

89 So aber Diedericbsen, Festschrift Schreiber (Fn. 30), S. 635 (648)

90 Vgl. Kobte, AcP 185 (1985), 105 (137 ff.); Baumann/Hartmann, DNotZ 2000, 594 (607);

Deutsch/Spickhoff (Fn. 31), Rn. 197; Roth, JZ 2004, 494 (496 f.)

91 Roth, JZ 2004, 494 (497); Lipp, FamRZ 2004, 317 (320); EK (Fn. 20), S. 18. Die Notwendigkeit, dass der Wille des Patienten geäußert worden bzw. erkennbar ist, betont zu Recht RPf(Fn. 20), S. 35

92 Vgl. dazu die Nachweise bei Fn. 85

93 Zu kritisch gegenüber Formularen deshalb Roth, JZ 2004, 494 (498)

94 Vgl. die Handreichungen der BÄK zum Umgang mit Patientenverfügungen, DÄBl. 1999, A 2720, Ziff. 1.1. und 3.3.; Verrel, MedR 1999, 547 (548 f.)

95 Zur Notwendigkeit der Auslegung Palandt/Diederichsen (Fn. 79), vor $\ 1896$ BGB Rn. 9; Röver (Fn. 80), S. 162; Roth, JZ 2004, 494 (498 ff.); EK (Fn. 20), S. 18

96 Roth, JZ 2004, 494 (500 ff.) 
solche beschränken, d.h. sie dürfen die Patientenverfügung nicht einfach wörtlich nehmen. Sie müssen vielmehr danach fragen, was der Patient damit erklären wollte. Bei dieser Feststellung des Patientenwillens müssen sie alle Informationen über den Patienten berücksichtigen, die ihnen bekannt geworden sind, und sich darüber hinaus, soweit dies in der konkreten Situation möglich ist, weitere Informationen verschaffen. Vor diesem Hintergrund haben sie dann die Patientenverfügung auszulegen $^{97}$. Enthält ein Dokument neben der antizipierten Erklärung der Einwilligung in bestimmte Maßnahmen oder ihrer Ablehnung auch Mitteilungen über persönliche Wertvorstellungen, Lebenseinstellungen usw., sind diese als Auslegungshilfe deshalb ebenso heranzuziehen wie die Auskünfte von Angehörigen oder des Hausarztes ${ }^{98}$.

\section{Patientenverfügung und Vertreter des Patienten}

In allen eingangs geschilderten Fällen lag nicht nur eine Patientenverfügung vor ${ }^{99}$, sondern die Patienten hatten auch jeweils einen Betreuer als gesetzlichen Vertreter (§ 1902 BGB). Das wirft zwei Fragen auf: Erstens, ob das VormG einen Betreuer überhaupt bestellen darf, und zweitens, ob ein Betreuer oder ein Bevollmächtigter an der Behandlung beteiligt werden muss, wenn der Patient eine Patientenverfügung verfasst hat ${ }^{100}$.

\subsection{Bestellung eines Betreuers trotz Patientenverfügung?}

Hat der Patient in einer Patientenverfügung seine Einwilligung bzw. seine Ablehnung bereits erklärt, ist die Bestellung eines Betreuers insoweit nicht erforderlich (\$ 1896 II 2 BGB). Notwendig bleibt die Betreuung jedoch für alle anderen, nicht in der Patientenverfügung vorweggenommenen Erklärungen und Entscheidungen, also z.B. für die Entscheidung, in welchem Krankenhaus und von welchem Arzt der Patient behandelt werden soll, für die vermögensrechtliche Seite der Behandlung usw. ${ }^{101}$ Die Patientenverfügung macht daher die Bestellung eines Betreuers in den meisten Fällen auch dann nicht entbehrlich, wenn sie eine in der konkreten Behandlungssituation einschlägige antizipierte Einwilligung oder Ablehnung des Patienten enthält102.

\footnotetext{
97 Zu den Auslegungsgrundsätzen Roth, JZ 2004, 494 (499 ff.)

98 Vgl. KK (Fn. 20), S. 23 f.

99 Vgl. BVerfG NJW 2002, 206; BGHZ 154, 205; zum Traunsteiner Fall BGH NJW 2005, 2385 und LG Traunstein NJW-RR 2003, 221; Putz, Die Schwester/Der Pfleger 2003, 226 (227). Allerdings wird der Inhalt der Patientenverfügung nicht mitgeteilt. Es ist daher offen, ob sie eine eigene Erklärung des Patienten enthält. Das Berufungsurteil des OLG München FamRZ 2003, 557 ff. erwähnt die Patientenverfügung nicht.

100 Vgl. zur rechtspolitischen Diskussion Lipp, Patientenautonomie (Fn. 1), S. 39 f.

101 Lipp, BtPrax 2002, 47 (51 f.); Diederichsen, Festschrift Schreiber (Fn. 30), S. 648 mit Fn. 88. Das übersieht z.B. Vosseler, BtPrax 2002, 295 (296)

$102 \mathrm{Zu}$ pauschal daher EK (Fn. 20), S. 44: Ein Betreuer sei stets zu bestellen.
} 
Notwendig wird die Bestellung eines Betreuers auch für die Erklärung der Einwilligung selbst, wenn der Arzt die Patientenverfügung und die darin enthaltenen antizipierten Erklärungen zwar kennt, aber Zweifel an ihrer Gültigkeit oder Anwendbarkeit hat und sich deshalb nicht an diese Erklärung des Patienten halten will. Erweisen sich diese Bedenken als berechtigt, muss ein Vertreter des Patienten über die Einwilligung in die Behandlung entscheiden. Meint der Arzt, dass der Patient seine Ansicht geändert hat, muss er daher beim VormG die Bestellung eines Betreuers anregen. In Eilfällen kann das VormG nach \1846 BGB selbst entscheiden. Nur falls auch dies zu spät käme, darf der Arzt die von ihm für notwendig erachtete Maßnahme - freilich auf sein Risiko - sofort durchführen ${ }^{103}$.

\subsection{Beteiligung eines Vertreters an der Behandlung?}

Hat der Patient eine Vertrauensperson formgerecht ( $\int 1904$ II BGB) bevollmächtigt, wird diese Vollmacht in aller Regel alle Behandlungsentscheidungen umfassen. Ebenso wird das VormG einem Betreuer regelmäßig die Gesundheitssorge zuweisen, ohne die in der Patientenverfügung genannten Fälle hiervon auszuschließen. Der Aufgabenkreis der Gesundheitssorge wird demnach regelmäßig sowohl die Entscheidung einschließen, ob lebensverlängernde Maßnahmen eingeleitet oder fortgesetzt werden sollen ${ }^{104}$, als auch die Durchsetzung einer entsprechenden Patientenverfügung.

Das geschieht nicht nur aus praktischen Gründen, sondern ist im Falle der Bestellung eines Betreuers auch mit dem Erforderlichkeitsgrundsatz des \1896 II 1 BGB vereinbar. Wie der Traunsteiner Fall zeigt, müssen diese Behandlungssituationen vom Aufgabenkreis des Betreuers umfasst sein, damit er prüfen kann, ob die Patientenverfügung noch dem Willen des Patienten entspricht, und damit er sie dann ggf. gegenüber Arzt, Pflegepersonal, Klinik oder Pflegeheim durchsetzen kann ${ }^{105}$.

Ein Vertreter in Gesundheitsangelegenheiten hat jedoch nicht nur die Aufgabe, einer vom Arzt vorgeschlagenen Behandlung zuzustimmen oder sie abzulehnen. Er hat darüber hinaus die Rechte und Interessen des Patienten im gesamten Behandlungsprozess wahrzunehmen, also bereits bei der einleitenden Untersuchung und Diagnose sowie bei der Erarbeitung der Indikation. Dementsprechend ist er von Anfang an am Behandlungsprozess zu beteiligen, und zwar auch dann, wenn eine Patientenverfügung vorliegt.

\footnotetext{
103 Lipp, in: Passive Sterbehilfe (Fn. 53), S. 54 f.; Taupitz (Fn. 1) A 120

104 BGH NJW 2005, 2385 (2385 f.); BGHZ 154, 205 (214); Diederichsen, Festschrift Schreiber (Fn. 30), S. 646; MünchKomm/Schwab (Fn. 53), \1904 BGB Rn. 38. Es geht daher nicht um einen Aufgabenkreis „Sterbehilfe“, wie manche meinen, vgl. z.B. Damrau/Zimmermann, Betreuungsrecht, 3. Aufl. 2001, J 1904 BGB Rn. 42; Stackmann, MedR 2003, 490 (493 f.); Wagenitr, FamRZ 2005, 669 (672)
}

105 Vgl. BGH NJW 2005, 2385 (2385) 
Teilweise wird der Vertreter jedoch generell für ungeeignet gehalten, den Willen und die Interessen des Patienten sachgerecht wahrzunehmen ${ }^{106}$. Abgesehen davon, dass pauschales Misstrauen hier ebenso wenig angebracht ist wie blindes Vertrauen, führt jedenfalls kein Weg daran vorbei, dass eine andere Person anstelle des einwilligungsunfähigen oder gar bewusstlosen Patienten dessen Rechte und Interessen im Behandlungsprozess wahrnehmen muss. Die Frage lautet daher nicht, ob jemand den Patienten vertreten soll, sondern wer. Wenn es keinen besonderen Vertreter des Patienten gäbe, bliebe allein der Arzt. Damit würden jedoch die Rechte des Patienten nicht gestärkt, sondern der Einsicht desjenigen überantwortet, gegen den sie u.U. geltend gemacht werden müssten. Für die Regelungen des geltenden Rechts, die für die Bestellung eines besonderen Vertreters Sorge tragen, sprechen daher die besseren Gründe ${ }^{107}$.

\subsection{Bedeutung der Patientenverfügung für den Vertreter}

Macht eine Patientenverfügung auch die Beteiligung eines Vertreters am Behandlungsprozess nicht entbehrlich, so hat sie für den Vertreter und seine stellvertretende Entscheidung zentrale Bedeutung. Hat der Patient in seiner Patientenverfügung die Entscheidung über die Behandlung schon getroffen, ist der Vertreter an diesen Willen des Patienten gebunden. Für den Bevollmächtigten ergibt sich dies aus $\int 665$ BGB $^{108}$, für den Betreuer hat dies der BGH in der Entscheidung vom 17.3.2003 ausdrücklich klargestellt ${ }^{109}$. Die so genannte „Wohlschranke“ des \ 1901 III 1 BGB greift hier also nicht ein ${ }^{110}$.

Dieser Wille des Patienten muss allerdings erst noch verwirklicht werden, indem z.B. Arzt und Pflegepersonal über die Patientenverfügung informiert oder zu ihrer Beachtung aufgefordert werden ${ }^{111}$. Der BGH beschreibt deshalb die Aufgabe des Betreuers zutreffend damit, dass er „dem Willen des Betroffenen gegenüber Arzt und Pflegepersonal in eigener rechtlicher Verantwortung (...) Ausdruck und Geltung zu verschaffen" hat ${ }^{112}$. Entsprechendes gilt für den Bevollmächtigten, der die Patientenverfügung als Weisung des Patienten umzusetzen hat ${ }^{113}$. Dementsprechend müssen Betreuer wie Bevollmächtigter mit haftungs- und strafrechtlichen Folgen rechnen, falls sie gegen diese Verpflichtung verstoßen. Darüber hin-

\footnotetext{
106 Statt vieler vgl. Duttge (Fn. 1), S. 40 ff., insbes. S. 41 („trügerische Hoffnung, dass der Vertreter sich an den Willen des Patienten halte").

107 Zum Vorstehenden ausführlich Lipp, Freiheit und Fürsorge (Fn. 39), S. 48 ff.

108 Vgl. Bamberger-Roth/Czub, BGB, 2003, 』 665 BGB Rn. 1

109 BGHZ 154, 205 (217); vgl. Schwab, Festschrift Henrich (Fn. 75), S. 517 f.; Bienwald, MDR 2003, 694; Baumann/Hartmann, DNotZ 2000, 594 (609 f.)

110 Lipp, DRiZ 2000, 231 (234); i.E. ebenso Roth, JZ 2004, 494 (495 in Fn. 14 a.E.)

111 Vgl. dazu aus klinischer Sicht Strätling/Sedemund-Adib/Bax/Scharf/Fieber/Schmucker(Fn. 13), 28 ff.

112 BGHZ 154, 205 (211). Ähnlich: BGH NJW 2005, 2385 (2385)

113 Zur Weisung vgl. nur Palandt/Sprau (Fn. 79), \665 BGB Rn. 1 f.; Bamberger-Roth/Cqub (Fn. 108), \ 665 BGB Rn. 1
} 
aus kann das VormG gegenüber dem Betreuer Aufsichtsmaßnahmen ergreifen (\$S 1908i, 1837, 1908b BGB).

$\mathrm{Zu}$ Recht stellt deshalb der 12. Zivilsenat des BGH in der Entscheidung vom 8.6.2005 fest, dass der Vertreter den Patienten vertritt ${ }^{114}$. Seine Anordnungen und Erklärungen sind für den Arzt, das Pflegepersonal etc. bindend. Eine eigene Prüfungskompetenz der Ärzte und Pflegekräfte besteht nicht. Ein „Durchgriff“ auf die Patientenverfügung als Erklärung des Patienten oder als Indiz für den mutmaßlichen Willen des Patienten sind dem Arzt und allen anderen grds. verwehrt. Diese Bindung findet nach allgemeinen Grundsätzen des Rechts der (gesetzlichen) Vertretung ihre Grenze bei einem Missbrauch der Vertretungsmacht, d. h. wenn der Vertreter sich nicht an den Willen des Patienten hält und dies für den Arzt evident ist. Ist dies nicht offensichtlich, besteht aber der Verdacht, dass der Betreuer seine Pflichten verletzt, insbesondere sich nicht an den Willen des Patienten hält, kann jedermann - also auch der Arzt - das VormG anrufen, das entweder einen Vollmachts- bzw. Kontrollbetreuer einsetzen ( $\int 1896$ III BGB) oder in Eilfällen selbst tätig werden kann (\$S 1908 i I 1, 1846 BGB).

In der Missachtung der Patientenverfügung der Zeugin Jehovas sowohl durch den Ehemann als Betreuer als auch durch die Ärzte liegt daher die eigentliche Problematik dieses Falles. Falls der Betreuer keine Anhaltspunkte für eine Willensänderung der Patientin hatte (vgl. \1901 III 2 BGB) ${ }^{115}$, handelte er pflichtwidrig, als er der Bluttransfusion zustimmte. Da die Ärzte die Patientenverfügung kannten, war für sie diese Pflichtwidrigkeit offensichtlich. Sie durften sich daher auf die Vertretungsmacht des Ehemanns nicht verlassen ${ }^{116}$ und behandelten die Patientin somit gegen deren erklärten Willen ${ }^{117}$.

\subsection{Mutmaßlicher Wille und subjektives Wohl des Patienten}

Fehlt es an einer Erklärung des Patienten gegenüber dem Arzt, etwa weil die Patientenverfügung keine antizipierte Erklärung über die vorgesehene Maßnahme enthält, sondern nur allgemeine Wünsche oder Wertvorstellungen mitteilt, muss der Vertreter selbst über die Einwilligung in die Aufnahme oder Fortführung der lebenserhaltenden Maßnahme entscheiden. Dabei haben sich der Bevollmächtigte nach \ 665 BGB, der Betreuer gemäß \ 1901 III BGB nach den Wünschen des Pati-

114 BGH NJW 2005, 2385 (2385); vgl. auch Wagenitr, FamRZ 2005, 669 (672)

115 Zu dieser Problematik Lipp, DRiZ 2000, 231 (234 mit Fn. 36); Schwab, Festschrift Henrich (Fn.

75), S. $517 \mathrm{f}$.

116 Nach den für einen Missbrauch der gesetzlichen Vertretungsmacht geltenden Regeln, vgl.

MünchKomm/Schwab (Fn. 53), \ 1901 BGB Rn. 20; Lipp, Freiheit und Fürsorge (Fn. 39), S. 184 ff., 187 f. Das übersieht das OLG München MedR 2003, 174 (178)

117 Die Entscheidung des Betreuers war jedoch nicht Gegenstand des Verfahrens vor den Vormundschaftsgerichten und damit auch nicht der Verfassungsbeschwerde. Das obiter dictum des BVerfG NJW 2002, 206 (207) ist gleichwohl i.E. zutreffend, weil der Betreuer kein an die Grundrechte gebundener Hoheitsträger ist (dazu Lipp, Freiheit und Fürsorge [Fn. 39], S. 118 ff.). Das übersieht die $E K$ (Fn. 20), S. 15, 37, wenn sie daraus ableitet, die Reichweite einer Patientenverfügung könne eingeschränkt werden. 
enten zu richten. Davon dürfen sie nur abweichen, falls der Wunsch zu sterben ausnahmsweise krankheitsbedingt ist ${ }^{118}$. Kennt der Vertreter die Wünsche des Patienten nicht, muss er die Angelegenheit mit dem Patienten besprechen, um ihm die Möglichkeit zur Selbstbestimmung zu erhalten ${ }^{119}$.

Nur wenn die Maßnahme dringend ist und nicht aufgeschoben werden kann, darf der Vertreter sofort entscheiden. Für den Bevollmächtigten ist dann nach $\$ 665 BGB der mutmaßliche Wille des Patienten maßgeblich ${ }^{120}$. Gleiches gilt bei der Notfallbehandlung für den Arzt ${ }^{121}$. Der mutmaßliche Wille des Patienten ist deshalb nicht mit dessen tatsächlich geäußertem Willen gleichzusetzen ${ }^{122}$. Es handelt sich vielmehr um einen Entscheidungsmaßstab für denjenigen, der anstelle des Patienten treuhänderisch dessen Selbstbestimmungsrecht ausübt'123. Für den Betreuer nennt $\int 1901$ II BGB als Ziel und Maßstab das vom Patienten her zu bestimmende, also das subjektive Wohl. Danach hat der Vertreter neben den Wünschen auch die Vorstellungen des Patienten, d.h. seine Lebensentscheidungen, Wertvorstellungen und Überzeugungen zu berücksichtigen. Soweit er sie nicht kennt, hat er ggf. Familienangehörige und andere Bezugspersonen danach zu fragen. Der Rückgriff auf die Interessenlage des Patienten ist ihm nur gestattet, wenn er die Wünsche und Vorstellungen des Patienten nicht feststellen kann ${ }^{124}$. Auch „,narrative“ oder nicht hinreichend konkrete Patientenverfügungen, die keine antizipierte Erklärung über die konkrete Behandlung gegenüber dem Arzt darstellen, sind daher vom Vertreter des Patienten bei seiner Entscheidung zu beachten, weil sie die subjektiven Wünsche und Vorstellungen des Patienten dokumentieren ${ }^{125}$.

Trotz der unterschiedlichen gesetzlichen Formulierungen für Beauftragte bzw. Bevollmächtigte einerseits und Betreuer andererseits handelt es sich demnach beim mutmaßlichen Willen und beim subjektiven Wobl des Patienten in der Sache um denselben Maßstab ${ }^{126}$. Der Vertreter hat sich daran zu orientieren, wie der Patient selbst entschieden hätte, d.h. an dessen (subjektiven) Wünschen und Vorstellun-

\footnotetext{
118 \665 S. 1 BGB (Abweichung von der Weisung im Interesse des Auftraggebers) bzw. \ 1901 III 1 BGB (Wohl des Betreuten); näher dazu Baumann/Hartmann, DNotZ 2000, 594 (608ff.); Lipp, BtPrax 2002, 47 (49) m.w.N.

119 \665 BGB bzw. \1901 III 3 BGB

120 Palandt/Sprau (Fn. 79), \665 BGB Rn. 7; Bamberger-Roth/Cqub (Fn. 108), \665 BGB Rn. 12

121 Vgl. nur Deutsch/Spickhoff (Fn. 31), Rn. 83

122 Insofern zutreffend Höfling/Rixen, JZ 2003, 884 (892 f.); Duttge (Fn. 1), S. 53 ff.

123 RPf (Fn. 20), S. 51 ff.; Lipp, FamRZ 2004, 317 (321 f.)

124 Dazu Hahne, FamRZ 2003, 1619 (1621); Lipp, BtPrax 2002, 47 (49 f.); anders Betreuungsrecht. Systematischer Praxiskommentar (BtKomm)/Roth, 2003, Teil D Rn. 11, der trotz \ 1901 II und III BGB allein auf das objektive Wohl abstellt. Der $B G H$ ließ ausdrücklich offen, wie hier zu entscheiden sei, $B G H Z$ 154, 205 (218 f.)

125 Röver (Fn. 80), S. 116; Schwab, Festschrift Henrich (Fn. 75), S. 518

126 Baumann/Hartmann, DNotZ 2000, 504 (609 f.); vgl. auch RPf(Fn. 20), S. 51 ff., wo allerdings nicht stets sorgfältig genug zwischen dem mutmaßlichen Willen als Entscheidungsmaßstab und der mutmaßlichen Einwilligung als Rechtfertigungsgrund für das ärztliche Handeln in Notfällen unterschieden wird (dazu sogleich im Text).
} 
gen und an dessen (objektiven) Interessen, nicht aber an den Wertvorstellungen der Allgemeinheit, wie der BGH zu Recht betont ${ }^{127}$.

Mangels einer antizipierten Erklärung des Patienten kann sich sein Vertreter nur an dessen Wünschen und an seinem subjektiv verstandenen Wohl orientieren, wie es \ 1901 II und III BGB für den Betreuer bzw. \ 665 BGB für den Bevollmächtigten vorschreiben. Die einzige Alternative dazu wäre eine Orientierung am rein objektiv verstandenen Wohl im Sinne einer Ausschöpfung aller medizinisch indizierten Maßnahmen. Diese Alternative hat der Gesetzgeber des Betreuungsgesetzes ausdrücklich abgelehnt und statt dessen die Regelung der \ 1901 II und III BGB eingeführt, um dem Betreuten ein größtmögliches $\mathrm{Maß}$ an Selbstbestimmung auch ,innerhalb“ einer Betreuung zu ermöglichen und damit den verfassungsrechtlichen Vorgaben zu genügen ${ }^{128}$. Die Kritik am mutmaßlichen Willen des Patienten ${ }^{129}$ ist daher in der Sache nicht gerechtfertigt. Soweit sie sich jedoch gegen die mutmaßliche Einwilligung als Rechtfertigungsgrund für das Handeln des Arztes richtet ${ }^{130}$, macht sie eindringlich klar, warum ein solches Handeln ohne Einschaltung eines Vertreters nur in Notfällen infrage kommen kann ${ }^{131}$.

Mutmaßlicher Wille und subjektives Wohl des Patienten sind daher letztlich ein und dasselbe. Sie drücken die Verpflichtung des Vertreters aus, aus Sicht des Patienten zu entscheiden. Sind die individuellen Präferenzen des Patienten weder bekannt noch zu ermitteln, kann sich der Vertreter nur an den Interessen des Patienten orientieren. Bei seiner Entscheidung, ob eine (weitere) lebenserhaltende Behandlung im Interesse des Patienten liegt, muss der Vertreter dessen erkennbare Interessen abwägen. Auch hier ist allerdings kein Raum für die Regel ,in dubio pro vita“, denn angesichts der Menschenwürdegarantie und ihres Primats kann eine solche Regel nur lauten: ,,in dubio pro dignitate“"132.

\section{Die Problematik der vormundschaftsgerichtlichen Genehmigung}

Damit stellt sich die weitere Frage, ob der Vertreter die Patientenverfügung ohne weiteres selbst umsetzen kann oder ob er dafür eine Genehmigung des VormG benötigt, wenn er lebenserhaltende Maßnahmen wie z.B. die künstliche Ernährung

\footnotetext{
127 BGH NJW 2005, 2385 (2385); BGHZ 154, 205 (218 f.); ebenso OLG Karlsrube FamRZ 2002, 488 (490, 492); RPf(Fn. 20), S. 51 ff., gegen BGHSt 40, 257 (263)

128 Lipp, Freiheit und Fürsorge (Fn. 39), S. 17 f., 149 ff.

129 Höfling/Rixen, JZ 2003, 884 (892 f.); Duttge (Fn. 1), S. 53 ff.; Wagenitr, FamRZ 2005, 669 (672)

130 Vgl. Höfling/Rixen, JZ 2003, 884 (892 nach Fn. 106) und Höfling, JuS 2000, 111 (116)

131 Dazu oben Abschnitt 4

$132 \operatorname{RPf}$ (Fn. 20), S. 54 f.
} 
des Komapatienten über eine PEG-Sonde ablehnt oder ihre Einstellung verlangt ${ }^{133}$.

\subsection{Genehmigungserfordernis für die Ablehnung einer lebenserhaltenden Maßnahme?}

Heftig umstritten ist dabei zunächst, ob das geltende Betreuungsrecht eine solche Genehmigung durch das VormG überhaupt vorsieht. Dies hatten mehrere Oberlandesgerichte im Einklang mit dem 1. Strafsenat des BGH entsprechend \ 1904 I BGB bejaht ${ }^{134}$. Demgegenüber hielten dies zahlreiche Untergerichte und auch das OLG Schleswig für ausgeschlossen, weil es dafür an einer Rechtsgrundlage fehle ${ }^{135}$. Der 12. Zivilsenat des BGH hat daraufhin auf Vorlage des OLG Schleswig entschieden, dass in bestimmten Fällen eine Genehmigung durch das VormG erforderlich ist. Dies begründet er jedoch nicht mit einer entsprechenden Anwendung des $\int 1904$ BGB, sondern gewinnt das Genehmigungserfordernis im Wege der Rechtsfortbildung ${ }^{136}$.

Aus diesem unterschiedlichen methodischen Weg ergeben sich jedoch keine Unterschiede in der Sache, weder in der sachlichen Begründung des Genehmigungserfordernisses noch in dessen praktischen Folgen ${ }^{137}$ : Eine Genehmigung des VormG ist erst erforderlich, wenn der Vertreter die Entscheidung über die lebenserhaltende Maßnahme trifft, d.h. der Arzt sie anbietet, weil sie aus seiner Sicht möglich und geboten ist, der Vertreter sie jedoch ablehnen möchte ${ }^{138}$. Umgekehrt ausgedrückt ist damit eine solche Genehmigung des VormG nicht erforderlich, wenn der Betreuer und der behandelnde Arzt sich gemeinsam gegen die Vornahme einer lebenserhaltenden Maßnahme entscheiden, weil in diesem Fall keine Konfliktsituation in Form eines Widerstreits von ärztlicher Empfehlung und Betreueranordnung vorliegt ${ }^{139}$.

Die Genehmigung ist eine Außengenehmigung, d.h. Voraussetzung für die Wirksamkeit der Entscheidung des Vertreters. Bis zur Genehmigung darf der Arzt

\footnotetext{
133 So lag der Sachverhalt im Traunsteiner Fall, vgl. BGH NJW 2005, 2385. Zur rechtspolitischen Diskussion um die vormundschaftsgerichtliche Genehmigung und zum Konsil Lipp, Patientenautonomie (Fn. 1), S. 47 ff.

134 BGHSt 40, 257 (261 f.); OLG Frankfurt a.M. NJW 1998, 2747 (2748) und FamRZ 2002, 577 (578); OLG Karlsrube FamRZ 2002, 488 (491); ebenso z.B. Deutsch/Spickhoff, Medizinrecht (Fn. 31), Rn. 511; Saliger, KritV 81 (1998), 118 (122 ff.); Taupitz (Fn. 1), A 87; Fröschle, JZ 2000, 72 (79 f.); Heyers (Fn. 44), S. 251 ff.; Lipp, in: Passive Sterbehilfe (Fn. 53), S. 49 ff.

135 OLG Schleswig FamRZ 2003, 554 ff.; ebenso z.B. Alberts, BtPrax 2003, 139 ff.; Wagenitr, FamRZ 2005, 669 (672); Schwab, Festschrift Henrich (Fn. 75), S. 523 f.

136 BGHZ 154, 205 (219 ff.); vgl. auch BGH NJW 2005, 2385 (2385 a. E.)

137 Letzteres sieht der 12. Zivilsenat ebenso - weshalb keine Anfrage beim 1. Strafsenat gem. \ 132 II, III 1 GVG erfolgte, vgl. BGHZ 154, 205 (228 f.). Die methodischen Fragen können im vorliegenden Zusammenhang deshalb dahinstehen; vgl. dazu z.B. Hufen, ZRP 2003, 248 f.

138 BGHZ 154, 205 (225 f.); bestätigt in BGH NJW 2005, 2385 (2385 a. E.); Lipp, in: Passive Sterbehilfe (Fn. 53), S. 52 f.

139 BGH NJW 2005, 2385 (2385 f.)
} 
deshalb die lebenserhaltende Maßnahme durchführen ${ }^{140}$. Dies beruht entweder auf einer weiterhin geltenden, weil nicht widerrufenen früheren Einwilligung oder, z.B. wenn die Behandlung erstmals aufgenommen werden soll, auf der mutmaßlichen Einwilligung des Patienten bzw. einer Entscheidung des VormG nach \1846 $\mathrm{BGB}$, weil der Betreuer an einer Entscheidung gehindert ist ${ }^{141}$.

Das Genehmigungserfordernis dient - wie \ 1904 BGB ${ }^{142}$ - der präventiven Kontrolle des Vertreters und damit dem Schutz des Selbstbestimmungsrechts des Patienten $^{143}$. Zugleich schafft die Genehmigung Rechtssicherheit für den Vertreter und die anderen Beteiligten, namentlich für den Arzt und das Pflegepersonal ${ }^{144}$. Das Genehmigungsverfahren unterliegt denselben Regeln wie die Genehmigung nach \1904 BGB145: Zuständig ist der Richter (\ 14 I Nr. 4 RPflG), der sich vom Zustand des Betroffenen einen persönlichen Eindruck zu verschaffen (\69d I 2 FGG) und ein Sachverständigengutachten einzuholen hat ( $\$$ 69d II FGG). Das VormG hat dabei allein die Rechtmäßigkeit der Entscheidung des Vertreters zu prüfen, also insbesondere, ob die weitere Behandlung dem erklärten oder mutmaßlichen Willen des Patienten widerspricht ${ }^{146}$.

Das Genehmigungserfordernis beschränkt allein die Rechtsmacht des Vertreters. Es ist daher kein Eingriff in das Grundrecht des Patienten auf Leben oder in sein Selbstbestimmungsrecht ${ }^{147}$. Vielmehr dient es dem Schutz des Patienten und seiner Grundrechte durch ein Verfahren, in dem der Wille des Patienten durch ein neutrales Gericht festgestellt wird ${ }^{148}$. Es gewährleistet damit, dass eine medizinisch indizierte lebenserhaltende Maßnahme nur mit dem Willen des Patienten eingestellt wird. Damit schützt es ihn zugleich davor, dass der Vertreter seine Entscheidungsbefugnis über Leben und Tod missbraucht, indem dem Patienten z.B. ein Sterbewunsch nur unterstellt und eine lebenserhaltende Maßnahme aus sachfremden Gründen vorenthalten wird.

\footnotetext{
140 BGHZ 154, 205 (225 f.)

141 Siehe z.B. Wagenitz/Engers, FamRZ 1998, 1256 (1257); Steffen, NJW 1996, 1581; Fröschle, JZ 2000, 72 (80). Die Ansicht des BGH, dies ergebe sich bereits aus der Genehmigungspflicht, erspart dem VormG lediglich eine besondere Anordnung zu Beginn des Genehmigungsverfahrens.

142 OLG Karlsrube FamRZ 2002, 488 (491); OLG Frankfurt a.M. FamRZ 2002, 577 (578); Lipp, in: Passive Sterbehilfe (Fn. 53), S. 49 ff.; Saliger, JuS 1999, 16 (18); Deutsch/Spickhoff(Fn. 31), Rn. 512

143 BGHZ 154, 205 (216 f., 223, 227). Das dort neben dem Selbstbestimmungsrecht genannte Grundrecht auf Leben und die Menschenwürde haben keine eigenständige Bedeutung, denn sie gewährleisten ebenfalls die Selbstbestimmung des Patienten über seine Behandlung am Lebensende, vgl. Hufen, NJW 2001, 849 (851 f.); Höfling, JuS 2000, 111 (114)

144 BGHZ 154, 205 (218 f.: Arzt, Pflegepersonal, 227: Betreuer); ebenso z.B. OLG Karlsruhe FamRZ 2002, 488 (490); Bauer, BtPrax 2002, 60 (62)

145 Zum folgenden BGHZ 154, 205 (224 ff.)

146 BGHZ 154, 205 (225 f.); ebenso OLG Karlsrube FamRZ 2002, 488 (491); Lipp, in: Passive Sterbehilfe (Fn. 53), S. 52; Taupitz.(Fn. 1), A 90 f.; Fröschle, JZ 2000, 72 (79 f.)

147 So aber z.B. Alberts, BtPrax 2003, 139 (140); Karliczek, FamRZ 2002, 578 (579); Storr, MedR 2002, 436 (439)

148 BGHZ 154, 205 (223); ebenso OLG Frankfurt FamRZ 2002, 575 (577); Saliger, KritV 1998, 118 (125, 134); Taupitz. (Fn. 1), A 82 f.; Lipp, in: Passive Sterbehilfe (Fn. 53), S. 52 f.
} 
Verfassungsrechtlich problematisch ist jedoch, dass auch derjenige Patient während des Genehmigungsverfahrens (weiter-) behandelt wird, bei dem sich später herausstellt, dass er die Behandlung ablehnt. Dieser Eingriff in seine Patientenautonomie ist notwendig mit dem obligatorischen Genehmigungsverfahren verbunden. Er wird allerdings gerechtfertigt durch die Notwendigkeit, den vom Vertreter zunächst nur behaupteten Willen des Patienten in einem gerichtlichen Verfahren festzustellen, wenn gerade diese Behauptung (z.B. vom Arzt) bestritten wird ${ }^{149}$.

Der BGH hat die Genehmigung im Falle des Betreuers nicht aus \ 1904 BGB abgeleitet, sondern aus einer „Gesamtschau des Betreuungsrechts“. Demzufolge ist unklar, ob dasselbe auch für den Bevollmächtigten gilt, der nur durch \ 1904 II BGB einer Genehmigungspflicht bei Behandlungsentscheidungen unterworfen wird. Da die Lösung des BGH auf eine analoge Anwendung des \ 1904 BGB hinausläuft und die angeführten Gründe für eine Genehmigung auch dann Geltung beanspruchen, wenn ein Bevollmächtigter den Patienten vertritt, dürfte für den Bevollmächtigten letztlich nichts anderes gelten.

\subsection{Genehmigung nur im Konfliktfall?}

Umstritten sind, in welchen Fällen eine Genehmigung des VormG eingeholt werden muss ${ }^{150}$. Der BGH hatte 2003 zunächst festgestellt, dass der Betreuer an eine Patientenverfügung gebunden ist. Danach führt er aus, das Genehmigungsverfahren eröffne die Möglichkeit der Feststellung, dass die gewünschte Einstellung der Behandlung dem in der Patientenverfügung niedergelegten Willen des Patienten entspreche. Das VormG müsse dann die Einstellung der Behandlung genehmigen $^{151}$. Abschließend heißt es allgemein, der Genehmigungsvorbehalt sei auf Konfliktfälle beschränkt, in denen der Betreuer die vom Arzt angebotene Behandlung ablehne ${ }^{152}$. Diese Position hat er in seinem jüngsten Beschluss vom 8.6.2005 präzisiert $^{153}$.

Akzeptiert der Arzt seinerseits die Patientenverfügung als verbindliche Erklärung des Patienten über die von ihm an sich für geboten erachtete Behandlung, liegt der vom BGH vorausgesetzte Konfliktfall zwischen Arzt und Vertreter eindeutig nicht vor. Da der Patient seinen Willen bereits erklärt hat und niemand Zweifel an dessen Geltung äußert, ist eine Genehmigung weder aus rechtlichen noch aus praktischen Gründen erforderlich ${ }^{154}$. Gleiches gilt, wenn zwar keine

\footnotetext{
149 Hufen, ZRP 2003, 248 (251 f.); diese Rechtfertigung übersieht Alberts, BtPrax 2003, 139 (140)

150 Hufen, ZRP 2003, 248 (250 f.); Kutzer, ZRP 2003, 213 f.; Paebler, BtPrax 2003, 141 (143); Rossbruch, PflR 2003, 254 (255 f.); Alberts, BtPrax 2003, 139 (140). Auch ich habe den Beschluss zunächst in dieser Weise verstanden (Lipp, FamRZ 2003, 756).

151 BGHZ 154, 205 (218)

152 BGHZ 154, 205 (227)

153 BGH NJW 2005, 2385 (2385f.)

154 Lipp, FamRZ 2003, 756; Strätling/Sedemund-Adib/Bax/Scharf/Fieber/Schmucker (Fn. 13), 27; Habne, FamRZ 2003, 1619 (1622); vgl. schon Scheffen, ZRP 2000, 313 (315); kritisch demgegenüber Verrel, NStZ 2003, 449 (450)
} 
antizipierte Erklärung des Patienten vorliegt, Arzt und Vertreter jedoch darin übereinstimmen, dass der Patient der lebenserhaltenden Maßnahme nicht zugestimmt hätte. Auch hier fehlt es an einem Konflikt über den letztlich entscheidenden mutmaßlichen Willen des Patienten ${ }^{155}$. Damit besteht kein Grund, der die vorläufige Behandlung während eines Genehmigungsverfahrens rechtfertigen würde. Ein Genehmigungserfordernis wäre in diesem Fall ein verfassungswidriger Eingriff in die Patientenautonomie, weil es zwangsläufig eine Behandlung gegen den Willen des Patienten während der Dauer des Genehmigungsverfahrens zur Folge hat ${ }^{156}$.

Hält der Arzt die Patientenverfügung dagegen - anders als der Vertreter - für unwirksam oder für nicht einschlägig, dann kommt es zum Konflikt. Damit wird ein Verfahren erforderlich und die damit verbundene Verzögerung in der Verwirklichung des Patientenwillens unvermeidbar. Die Behandlung des Patienten während des Verfahrens ist verfassungsrechtlich unbedenklich ${ }^{157}$, weil gerade der Wille des Patienten im Streit ist. Dem BGH ist deshalb darin zuzustimmen, dass hier ein Genehmigungsverfahren erforderlich und auf Antrag des Vertreters einzuleiten ist ${ }^{158}$.

Hat der Patient selbst die lebenserhaltende Maßnahme im Wege einer antizipierten Erklärung abgelehnt und versucht der Vertreter lediglich, diese Erklärung durchzusetzen, ist für eine materielle Genehmigungspflicht jedoch kein Raum ${ }^{159}$. Der Vertreter ruft hier das VormG nur deshalb an, weil er eine Chance sieht, den Willen des Patienten auf diesem Weg schneller und einfacher zu verwirklichen als mit einer Klage vor den allgemeinen Zivilgerichten ${ }^{160}$. Der Unterschied zwischen der auch hier bestehenden Möglichkeit eines Genehmigungsverfahrens und der abzulehnenden Pflicht des Betreuers, eine Genehmigung einzuholen, wird bedeutsam, wenn der Vertreter die antizipierte Erklärung auf andere Weise durchzusetzen versucht. Wäre eine Genehmigung zwingend vorgeschrieben, würde der Vertreter sowohl pflichtwidrig als auch ohne Vertretungsmacht handeln, wenn er etwa eine Unterlassungsklage vor den Zivilgerichten erhebt.

Fraglich war bisher auch, ob auf Antrag des Vertreters ein Verfahren einzuleiten ist. Im Beschluss vom 8.6.2005 hat der BGH ausdrücklich klargestellt, dass das VormG nur dann zu einer Entscheidung berufen ist, wenn der einen einwilligungsunfähigen Patienten behandelnde Arzt eine lebenserhaltende oder -verlän-

\footnotetext{
155 Stackmann, MedR 2003, 490 (496 unter c.). Im Traunsteiner Fall stand der Wille des Patienten außer Streit (LG Traunstein, NJW-RR 2003, 221), wurde also auch von Pflegepersonal und Heim nicht bezweifelt. Eine Genehmigung des VormG war deshalb entbehrlich.

156 Hufen, ZRP 2003, 248 (251 f.); Alberts, BtPrax 2003, 139 (140)

157 Hufen, ZRP 2003, 248 (251 f.)

158 BGHZ 154, 205 (210, 227); BGH NJW 2005, 2385 (2385 a. E.); Borasio/Putz/Eisenmenger, DÄBl. 2003, A 2062 (2064); Habne, FamRZ 2003, 1619 (1621 f.)

159 Das VormG kann nur die Erklärung des Betreuers genehmigen, nicht die des Patienten; vgl. auch Höfling/Rixen, JZ 2003, 884 (891 f.); Lipp, FamRZ 2003, 756

160 Dazu sogleich Abschnitt 8
} 
gernde Maßnahme für medizinisch geboten oder vertretbar erachtet und sie „anbietet", der Betreuer dieses Angebot aber ablehnt ${ }^{161}$. Im Traunsteiner Fall hatten sich aber sowohl der Betreuer als auch der behandelnde Arzt gegen eine weitere künstliche Ernährung entschieden. Lediglich das Pflegeheim wollte die künstliche Ernährung fortsetzen. Dieser Fall fällt nach dem BGH aber zu Recht nicht unter die Kontrollzuständigkeit des VormG.

\section{Durchsetzung der Patientenverfügung}

Der Traunsteiner Fall zeigt, dass das vormundschaftsgerichtliche Genehmigungsverfahren kein Königsweg zur Durchsetzung einer Patientenverfügung ist. Auch wenn das VormG bestätigt, dass die Patientenverfügung maßgeblich ist, werden dadurch Arzt und Pflegepersonal, Klinik und Pflegeheim nicht gerichtlich verpflichtet, z.B. die künstliche Ernährung durch eine PEG-Sonde zu unterlassen ${ }^{162}$. Mit anderen Worten: Die Entscheidung des VormG verschafft dem Patienten bzw. dem Vertreter keinen vollstreckbaren Titel gegenüber Arzt, Pflegepersonal, Klinik oder Pflegeheim. Dazu muss der Vertreter die Rechte des Patienten vor den allgemeinen Zivilgerichten verfolgen ${ }^{163}$. Seine Befugnis hierzu folgt aus der Befugnis zur Durchsetzung der Patientenverfügung, ist also ebenfalls Bestandteil des die fragliche Behandlung umfassenden Aufgabenkreises (Betreuer) bzw. der Vollmacht ${ }^{164}$. Eine Genehmigung ist nach dem eben Gesagten nur erforderlich, wenn ein Konflikt hinsichtlich des letztlich allein maßgeblichen Willens des Patienten besteht ${ }^{165}$. Streiten die Parteien - wie im Traunsteiner Fall ${ }^{166}$ - nicht über den Willen des Patienten, sondern über andere Fragen, ist das Genehmigungsverfahren deshalb nicht vorgreiflich ${ }^{167}$.

Sowohl im Schmerzensgeldprozess der Zeugin Jehovas als auch im Traunsteiner Fall hatten sich die beklagten Ärzte und die Klinik bzw. das Pflegeheim darauf berufen, sie müssten die Patientenverfügung bzw. die Anweisung des Betreuers nicht befolgen, weil es ihrem Gewissen widerspreche, einen Patienten verhungern

\footnotetext{
161 BGH NJW 2005, 2385 (2385 f.). Dagegen noch unklar, da nicht entscheidungserheblich: BGHZ 154, $205(210,227)$

162 Insoweit zutreffend OLG München FamRZ 2003, 557 (559)

163 Eine ärztliche Maßnahme gegen den Willen des Patienten verletzt seinen Körper bzw. sein allgemeines Persönlichkeitsrecht und begründet neben den quasinegatorischen Unterlassungs- und Beseitigungsansprüchen analog \1004 I BGB und deliktischen Schadensersatzansprüchen nach \ 823 I BGB auch Schadensersatzansprüche wegen Verletzung des Behandlungs- bzw. Pflege- oder Heimvertrags gem. SS 280 I, 241 II, 249 I BGB, vgl. zur Arzthaftung nur Deutsch/Spickhoff (Fn. 31), Rn. 122 ff. (allgemein), S. 265 ff. (bei unwirksamer Einwilligung).

164 Ähnlich Höfling/Rixen, JZ 2003, 884 (890 f.)

165 A. A. Wagenity, FamRZ 2005, 669 (672), der sich dabei auf die Entscheidung des BGH vom 17.3.2003 (BGHZ 154, 205 (224 f.) stützt.

166 Oben Fn. 155

167 Insoweit zutreffend OLG München FamRZ 2003, 557 (559)
} 
zu lassen. Dem sind die Gerichte im Fall der Zeugin Jehovas und die Vorinstanzen im Traunsteiner Fall gefolgt ${ }^{168}$. Die Gerichte haben übersehen, dass es nicht um den Anspruch des Patienten auf die Durchführung einer ärztlichen Maßnahme (wie z.B. eines Schwangerschaftsabbruchs) geht, sondern um das Vetorecht des Patienten, die angebotene künstliche Ernährung abzulehnen ${ }^{169}$. Auch das Grundrecht der Gewissensfreiheit oder ihre Menschenwürde gibt Ärzten, Pflegenden oder den Einrichtungen, in denen sie arbeiten, kein Recht auf die künstliche Ernährung eines Patienten mittels einer PEG-Sonde gegen dessen Willen ${ }^{170}$. Durch die Entscheidung des 12. Zivilsenats des BGH vom 8.6.2005 im Traunsteiner Fall ist dies nun höchstrichterlich klargestellt ${ }^{171}$.

\section{Ausblick}

Seit dem Beschluss des BGH vom 8.6.2005172 dürfen viele Fragen als geklärt betrachtet werden, die sich im Zusammenhang der Einstellung lebenserhaltender Maßnahmen stellen. Eine gewisse Ausnahme gilt wegen der dort höchstrichterlich geäußerten Zweifel weiterhin für die so genannten „strafrechtlichen Grenzen der Sterbehilfe im weiteren Sinn“. Diese Zweifel sind zwar unbegründet, wie die Lektüre der einschlägigen Entscheidung des 1. Strafsenats des BGH zeigt ${ }^{173}$; die von der Praxis gewünschte Klarheit über die rechtlichen Grundlagen der Entscheidungsfindung dürfte sich jedoch erst nach einer weiteren höchstrichterlichen Entscheidung oder infolge eines klärenden Wortes des Gesetzgebers einstellen.

\footnotetext{
168 OLG München NJW-RR 2002, 811 - Zeugin Jehovas; OLG München FamRZ 2003, 557; Vorinstanz LG Traunstein NJW-RR 2003, 221 - Traunstein

169 Vgl. BGHZ 154, 205 (224 f.); Taupitz. (Fn. 1), A 23; und oben Abschnitt 3.1

170 Hufen, ZRP 2003, 248 (252)

171 BGH NJW 2005, 2385

172 BGH NJW 2005, 2385 ff.

173 Zu BGHSt 40, 257 ff. siehe oben bei Fn. 58
} 


\title{
Die Praxis der aktiven Sterbehilfe in den Niederlanden und Belgien
}

\author{
Alfred Simon
}

\section{Einleitung}

Seitdem der Schweizer Sterbehilfeverein Dignitas im September 2005 einen deutschen Verein mit Büro in Hannover eröffnet hat, ist die Diskussion über Sterbehilfe in Deutschland voll im Gange. Während zahlreiche Vertreter aus Politik, Kirche, Ärzteschaft und anderen gesellschaftlich einflussreichen Institutionen empört reagierten, rief der damalige Hamburger Justizsenator Roger Kusch dazu auf, neben der in Deutschland straffreien Beihilfe zum Suizid, auch die aktive Sterbehilfe unter bestimmten Voraussetzungen zu ermöglichen. Kusch verwies dabei auf eine Umfrage des Meinungsforschungsinstituts Forsa, nach der 74 Prozent der Deutschen eine Zulassung der aktiven Sterbehilfe befürworten. Eine von der Deutschen Hospizstiftung in Auftrag gegebene Umfrage des Meinungsforschungsinstituts Emnid kam hingegen zu einem ganz anderen Ergebnis: Dieser Umfrage zufolge befürworten nur 35 Prozent der Deutschen die aktive Sterbehilfe, während sich 56 Prozent für Palliativmedizin und Hospizarbeit aussprechen.

Ähnlich instrumentalisiert wie die öffentlichen Meinungsumfragen ist der Verweis auf die Sterbehilfepraxis in den Niederlanden und Belgien. In beiden 
Ländern sind seit einigen Jahren Gesetze in Kraft, die es Ärzten ermöglichen, das Leben schwerkranker Menschen auf deren Verlangen hin straffrei zu beenden. Während Befürworter der aktiven Sterbehilfe den offenen und transparenten Umgang mit dem Thema in diesen Ländern als vorbildlich loben, sehen Gegner ihre schlimmsten Befürchtungen bestätigt. Ein nüchterner Blick auf die gesetzlichen Regelungen und die Praxis der Sterbehilfe in den Niederlanden und Belgien zeigt jedoch, dass weder die kritiklose Befürwortung noch die pauschale Verurteilung angemessene Reaktionen darstellen.

\section{Die Praxis der aktiven Sterbehilfe in den Niederlanden}

Das niederländische Parlament hat im Frühjahr 2001 ein Gesetz beschlossen, das Ärzten ermöglicht, unter Einhaltung gesetzlich vorgeschriebener Sorgfaltskriterien straffrei aktive Sterbehilfe zu leisten. Damit wurde eine seit Jahren existierende und von den Gerichten offiziell geduldete Praxis strafrechtlich geregelt.

\subsection{Gesetzliche Regelung}

Das neue Gesetz regelt sowohl die Lebensbeendigung auf Verlangen als auch die Hilfe bei der Selbsttötung. Beide Handlungen bleiben strafbar, es sei denn, der Arzt hält die gesetzlich vorgeschriebenen Sorgfaltskriterien ein und meldet den nicht natürlichen Tod einer der regionalen Kontrollkommissionen.

Die Sorgfaltskriterien beinhalten, dass der Aržt

a) zu der Überzeugung gelangt ist, dass der Patient seine Bitte freiwillig und nach reiflicher Überlegung gestellt hat,

b) zu der Überzengung gelangt ist, dass der Zustand des Patienten aussichtslos und sein Leiden unerträglich ist,

c) den Patienten über seine Situation und über dessen Aussichten aufgeklärt hat, d) gemeinsam mit dem Patienten zu der Überzengung gelangt ist, dass es für dessen Situation keine andere annehmbare Lösung gibt,

e) mindestens einen anderen, unabhängigen Aržt zu Rate gezogen hat, der den Patienten untersucht und schriftlich zu den unter den Buchstaben a bis d genannten Sorgfaltskriterien Stellung genommen hat, und

f) bei der Lebensbeendigung oder der Hilfe zur Selbsttötung mit medizinischer Sorgfalt vorgegangen ist. ${ }^{1}$

Es gibt fünf Kontrollkommissionen, die die Einhaltung dieser Sorgfaltskriterien bei jedem gemeldeten Fall von Sterbehilfe überprüfen. Eine Kommission

\footnotetext{
1 zitiert nach: FAQ Sterbehilfe 2001, hrsg. von der Abteilung Auslandsinformation des Ministeriums für auswärtige Angelegenheiten in Zusammenarbeit mit dem Ministerium für Gesundheit, Gemeinwobl und Sport und dem Ministerium der Justiz in den Niederlanden.
} 
besteht aus einer ungeraden Zahl von Mitgliedern, darunter in jedem Fall ein Jurist, der zugleich Vorsitzender ist, ein Arzt und ein Ethiker. Die Mitglieder der Kommission werden vom Justiz- und vom Gesundheitsministerium gemeinsam für sechs Jahre ernannt.

Die Kontrollkommissionen sind bereits seit November 1998 tätig. Während ihr Urteil zunächst nur beratende Funktion für die Staatsanwaltschaft hatte, muss eine Kommission nach dem neuen Gesetz die Staatsanwaltschaft nicht mehr einschalten, wenn sie der Auffassung ist, dass die oben genannten Sorgfaltskriterien beachtet worden sind.

Die strafrechtliche Regelung der aktiven Sterbehilfe beruht auf einem Meldeverfahren, das bereits im Juni 1994 in die allgemeinen Verwaltungsanordnungen der Niederlande (Artikel 10 des Gesetzes über die Leichenbestattung) aufgenommen worden ist. Dieses sieht folgende Schritte vor:

- Der Arzt meldet seine Sterbehilfehandlung als „nicht natürlichen Tod“ dem zuständigen Leichenbeschauer und erstellt anhand eines vorgegebenen Fragenkatalogs einen detaillierten Bericht.

- Der Leichenbeschauer erstellt selbst einen Bericht und schickt diesen an den Staatsanwalt, der die Leiche zur Bestattung freigeben muss.

- Der regionalen Kontrollkommission gehen sowohl der Bericht des Arztes als auch der des Leichenbeschauers zu. Außerdem erhält sie die Erklärung des zweiten Arztes und, sofern vorhanden, die Patientenverfügung des Verstorbenen.

- Die Kontrollkommission überprüft die Handlungsweise des Arztes anhand der Sorgfaltskriterien. Gelangt sie zu der Überzeugung, dass der Arzt sorgfältig gehandelt hat, wird er nicht strafrechtlich verfolgt.

- Kommt die Kommission zu dem Ergebnis, dass der Arzt die Sorgfaltskriterien nicht eingehalten hat, teilt sie dies der Staatsanwaltschaft und der regionalen Gesundheitsbehörde mit. Beide Instanzen prüfen dann, ob und, wenn ja, welche Schritte gegen den Arzt unternommen werden müssen.

\subsection{Entwicklung der Sterbehilfepraxis}

Dem neuen Gesetz geht eine langjährige, und seit Mitte der 1980er Jahre von den Gerichten geduldete Sterbehilfepraxis voraus. Hier einige der wichtigsten Meilensteine in der Entwicklung dieser Praxis:

1973

Eine Ärztin muss sich vor Gericht verantworten, weil sie das Leben ihrer eigenen Mutter auf deren ausdrücklichen und wiederholten Wunsch hin beendet hat. Das Gericht in Leeuwarden würdigt die Motive der Ärztin, sieht sich aufgrund der 
Gesetzeslage jedoch genötigt, die Frau zu einer bedingten Haftstrafe zu verurteilen ${ }^{2}$.

\section{4}

Die niederländische Ärztekammer veröffentlicht ihren Standpunkt über Euthana$\mathrm{sie}^{3}$. In dem Dokument wird die Lebensbeendigung auf Verlangen nicht als eine unrechtmäßige Handlung betrachtet, sondern als eine Tat, die zu den Aufgaben eines Arztes gehört. Das Dokument enthält ferner Richtlinien für Ärzte, die mit dem Wunsch von Patienten nach Sterbehilfe konfrontiert werden. Die in diesem Zusammenhang genannten Sorgfaltskriterien entsprechen im Kern bereits denen, die nun im Strafgesetzbuch festgelegt wurden.

Im selben Jahr fällt das oberste Gericht der Niederlande ein richtungsweisendes Urteil, das die Grundlage für die in der Folge praktizierte Duldung von Sterbehilfehandlungen legt. Nach Ansicht des Hohen Rates stellt die Konfliktsituation, in der sich der Arzt aufgrund seiner Verpflichtung gegenüber dem Verlangen des Patienten auf der einen und seiner Verpflichtung gegenüber dem Gesetz auf der anderen Seite befindet, eine Form „höherer Gewalt“ dar. Das niederländische Strafrecht sieht vor, dass in einem solchen Fall von Bestrafung abgesehen werden kann ${ }^{4}$.

\section{5}

Die Staatskommission Euthanasie ${ }^{5}$ spricht sich in ihrem Bericht für eine Legalisierung der Lebensbeendigung auf Verlangen und der Hilfe bei der Selbsttötung durch den Arzt aus. Die Kommission wurde 1982 auf Antrag des Gesundheitsund des Justizministeriums beauftragt, einen Gesetzesvorschlag für die Regelung von Sterbehilfehandlungen auszuarbeiten. Die Vorschläge der Staatskommission werden von einer Mehrheit im Parlament unterstützt, ihre Umsetzung scheitert aber am Widerstand der in Koalition mit den Konservativen regierenden Christdemokratischen Partei.

2 vgl. Leenen HJJ (1989): Dying with dignity: developments in the field of euthanasia in the Netherlands. Medicine and Law, 8, 517-526

3 Koninklijke Nederlandse Maatschappij tot bevordering van de Geneeskunst (KNMG) (1984): Standpunkt inzake euthanasie. Medisch Contact, 39, 990-998. Die englischsprachige Übersetzung ist u.a. abgedruckt in der von der KNMG herausgegebenen Textsammlung: Euthanasia in the Netherlands (1993): Utrecht, S. 8-22

4 vgl. Welie JVM (1992): The medical exception: physicians, euthanasia and the Dutch criminal law. The Journal of Medicine and Philosophy, 17, 419-437

5 Staatscommissie Euthanasie (1985): Rapport inzake euthanasia. Den Haag [Englische Zusammenfassung: Anonymus (1987): Final Report of the Netherlands State Commission on Euthanasia: an English Summary. Bioethics, 1, 163-174] 


\section{0 er Jabre}

Die Regierung beauftragt eine Kommission, die medizinische Vorgehensweise im Hinblick auf Entscheidungen am Lebensende zu untersuchen. Der Bericht der Remmelink-Kommission ${ }^{6}$ führt 1991 zu einem neuen Gesetzesvorschlag, der 1993 von beiden Kammern des niederländischen Parlaments angenommen wird und 1994 in Kraft tritt. Das Gesetz sieht keine Änderung des Strafrechtes vor. Im Wesentlichen wird das oben beschriebene Meldeverfahren, auf das sich Ärztekammer und Staatsanwaltschaft bereits 1990 geeinigt hatten, übernommen und dem Gesetz über die Leichenbestattung beigefügt, wodurch dieses den formellen Status eines Gesetzes erhält.

\subsection{Empirische Daten}

Die Kontrollkommissionen geben einen jährlichen Tätigkeitsbericht heraus ${ }^{7}$. Diesem Bericht zufolge wurden im Jahr 2004 insgesamt 1933 Sterbehilfehandlungen gemeldet. In 1765 Fällen ging es um Lebensbeendigung auf Verlangen, in 143 Fällen um Beihilfe zum Suizid und in 25 Fällen um eine Kombination von beidem. Die überwiegende Mehrzahl der durch aktive Sterbehilfe oder Beihilfe zum Suizid aus dem Leben geschiedenen Patienten litt an einer Tumorerkrankung ( $\mathrm{n}=$ 1713). In den allermeisten Fällen wurde die Sterbehilfehandlung durch den Hausarzt $(\mathrm{n}=1697)$ zu Hause beim Patienten ( $\mathrm{n}=1585)$ durchgeführt. In Krankenhäusern und Pflegeheimen hingegen kam Sterbehilfe nur sehr selten vor. In drei der insgesamt 1933 sah die Kontrollkommission die Sorgfaltskriterien als nicht erfüllt an; die Akten wurden an die Staatsanwaltschaft und den zuständigen Gesundheitsinspektor weitergeleitet.

Vergleicht man diese Zahlen mit den Ergebnissen einer landesweiten Studie aus dem Jahre 2001, die etwa 3500 Fälle von Lebensbeendigung auf Verlangen und weitere 300 Fälle von Beihilfe zum Suizid zu Tage brachte, so ist davon auszugehen, dass nur etwa die Hälfte der tatsächlichen Sterbehilfehandlungen den Kontrollkommissionen gemeldet werden.

Zwei aktuelle Entwicklungen verdienen ferner Beachtung: 2004 wurde erstmals ein Fall von Lebensbeendigung auf Verlangen bei einem Alzheimerpatienten gemeldet. Obwohl der Patient noch längere Zeit hätte leben können, sah die zuständige Kontrollkommission die Sorgfaltskriterien als erfüllt an. Anfang 2005 wurde bekannt, dass zwischen 1997 und 2004 insgesamt 22 Neugeborene mit Spina bifida oder Hydrozephalus auf Verlangen der Eltern getötet wurden. In allen Fällen wurden die Ermittlungen seitens der Staatsanwaltschaft eingestellt. Eine Gruppe von Pädiatern und Staatsanwälten hat bereits im Jahr 2002 das „Pro-

\footnotetext{
6 Benannt nach ihrem Leiter, dem Vorsitzenden des niederländischen Höchstgerichtes, Prof. J. Remmelink.

7 Regionale toetsingscommissies euthanasie (2005): Jaarverslag, http://www.toetsingscommissieseuthanasie.nl/Images/jaarverslagRTE2005_tcm9-1973.pdf (Stand: 12. Juli 2006)
} 
tokoll von Groningen" erarbeitet, in dem medizinische und rechtliche Kriterien für die aktive Sterbehilfe bei schwerkranken Neugeborenen benannt werden ${ }^{8}$. Pressemitteilungen zufolge plant das Niederländische Justizministerium ein Gesetz, das auch diese Form der Sterbehilfe unter bestimmten Voraussetzungen ermöglichen soll.

\section{Die Praxis der aktiven Sterbehilfe in Belgien}

Als zweites Land der Welt hat Belgien im Mai 2002 ein Gesetz beschlossen, das die Lebensbeendigung auf Verlangen durch Ärzte ermöglicht.

\subsection{Gesetzliche Regelung}

Das belgische Gesetz ist dem niederländischen in großen Teilen ähnlich: Wie in den Niederlanden ist ein freiwilliges, wohl überlegtes, andauerndes und unbeeinflusstes Verlangen des Patienten nötig. Das physische oder psychische Leiden des Patienten muss unerträglich, andauernd und unheilbar sein. Der Arzt muss sich vor Durchführung der Sterbehilfe mit einem zweiten unabhängigen Arzt beraten und die durchgeführte Handlung einer Bundeskommission melden.

Es gibt aber auch einige entscheidende Unterschiede: Zunächst ist der behandelnde Arzt verpflichtet, dem Patienten die Möglichkeiten der Palliativmedizin aufzuzeigen. Zudem ist er gehalten, Kontakt zu den Angehörigen und einem möglicherweise vorhandenen Pflegeteam aufzunehmen und mit diesen über das Begehren des Patienten zu sprechen. Ferner verlangt das Gesetz, dass der Wunsch nach Sterbehilfe schriftlich fixiert und zu den Krankenakten gelegt wird. Ist der Patient dazu nicht mehr in der Lage, so übernimmt dies eine volljährige Person seiner Wahl. Es besteht auch die Möglichkeit einer antizipierten Sterbehilfeerklärung. Der in der Öffentlichkeit am meisten wahrgenommene Unterschied besteht jedoch darin, dass der Patient nach belgischem Recht nicht in absehbarer Zeit sterben muss. Es genügt bereits, wenn er unheilbar krank ist, sein Tod aber zeitlich noch weit entfernt. In diesem Fall ist zwischen dem schriftlich dokumentierten Wunsch nach Sterbehilfe und der Durchführung derselben eine Wartefrist von einem Monat vorgeschrieben.

\subsection{Empirische Daten}

Im September 2004 hat die Bundeskommission für die Kontrolle und Evaluation des Euthanasie-Gesetzes erste Zahlen veröffentlicht: Demnach wurden von September 2002 bis Dezember 2003259 Fälle von Lebensbeendigung auf Verlangen gemeldet. In 91,5 Prozent dieser Fälle war der Patient als terminal, in 8,5 Prozent

$8 \quad$ Verhagen E \& Sauer PJJ (2005): The Groningen Protocol - Euthanasia in Severely Ill Newborns. N Engl J Med, 352;10, 959-962 
als nicht terminal eingestuft. 41 Prozent der Sterbehilfehandlungen wurden zu Hause beim Patienten, 54 Prozent in Krankenhäusern und 5 Prozent in Pflegeheimen durchgeführt. 80 Prozent der gemeldeten Fälle ereignete sich im flämischen Teil Belgiens, 20 Prozent im wallonischen. Dieses überraschende Ergebnis dürfte auf die kulturellen Unterschiede dieser beiden Landesteile zurückzuführen sein.

Nach einer Studie von Deliens et al. ${ }^{9}$ gab es 1998 ca. 640 Fälle von Lebensbeendigung auf Verlangen in Flandern. Da bis Dezember 2003 - also 15 Monate nach Inkrafttreten des Gesetzes - in ganz Belgien nur 259 Fälle gemeldet wurden, muss man davon ausgehen, dass - ähnlich wie in den Niederlanden - die Mehrzahl der Sterbehilfefälle von den belgischen Ärzten nicht gemeldet wird.

\section{Moralische Bewertung}

Die moralische Bewertung der Sterbehilfepraxis in den Niederlanden und Belgien hängt natürlich von der eigenen Wertvorstellung ab. Für den gesellschaftlichen Diskurs, dem sich - wie die einleitenden Beispiele gezeigt haben - auch Deutschland in zunehmenden Maße stellen muss, erscheint es sinnvoll, die individualethische Frage nach der moralischen Zulässigkeit der aktiven Sterbehilfe und der Beihilfe zum Suizid im Einzelfall und die sozialethische Frage nach den möglichen Folgen einer liberaleren Sterbehilfepraxis zu unterscheiden.

\subsection{Die individualethische Perspektive}

Jeder von uns hat ein Interesse daran, nicht von einem anderen Menschen getötet $\mathrm{zu}$ werden. Dieses Interesse ist so grundlegend, dass es durch ein grundgesetzlich festgeschriebenes Recht auf Leben geschützt wird. Dieses Recht kann durch kein anderes Interesse verdrängt werden: So darf ein Mensch im Normalfall auch dann nicht getötet werden, wenn nur durch die Tötung dieses Menschen - etwa unter Nutzung seiner Organe - mehrere andere Leben gerettet werden können.

Wie aber sieht es aus, wenn der Betroffene selbst die Beendigung seines Lebens wünscht? Das Recht auf Leben enthält keine Pflicht zu leben. Ebenso wie das aktive und das passive Wahlrecht keine Pflicht enthalten, zur Wahl zu gehen oder sich als Kandidat zur Wahl zu stellen, sondern nur die Aufforderung, verantwortungsvoll mit diesem Recht umzugehen, kann aus dem Recht auf Leben nur die moralische Aufforderung zu einem verantwortungsvollen Umgang mit dem eigenen Leben abgeleitet werden. Ob der wohlüberlegte Wunsch nach Beendigung des eigenen Lebens angesichts eines unheilbaren und vom Betroffenen selbst als unerträglich empfundenen Leidens dieser Aufforderung entspricht, kann in letzter Konsequenz nur vom Betroffenen selbst beurteilt werden.

9 Deliens L et al. (2000): End-of-life decisions in medical practice in Flanders, Belgium: a nationwide survey. The Lancet, 356, 1806-1811 
Wenn aber jemand beschlossen hat, sein Leben zu beenden, so stellt es auch keine Verletzung des Lebensrechts dar, wenn andere ihm auf seinen ausdrücklichen Wunsch hin bei der Durchführung seines Beschlusses helfen. Das Recht auf Leben schließt also die Möglichkeit der Suizidbeihilfe und der Tötung auf Verlangen nicht kategorisch aus (begründet umgekehrt aber auch keinen Anspruch auf Sterbehilfe).

\subsection{Die sozialethische Perspektive}

Die Tatsache, dass die Beihilfe zum Suizid und die Tötung auf Verlangen im Einzelfall moralisch zulässig sein können, bedeutet jedoch nicht, dass beide Formen der Sterbehilfe auch gesetzlich erlaubt sein sollen. Denn bei der Gesetzgebung sind über die individualethische Perspektive hinaus auch die sozialethischen Auswirkungen eines Gesetzes mit zu bedenken.

Mögliche Probleme einer liberaleren Sterbehilfegesetzgebung sind:

- das Problem der Authentizität: Verbirgt sich hinter dem Sterbehilfewunsch tatsächlich der Wunsch nach dem eigenen Tod oder drückt er nur aus, dass der Patienten unter den gegebenen Bedingungen (z.B. belastende Symptome, soziale Vereinsamung) nicht weiterleben möchte? Birgt folglich eine liberalere Sterbehilfegesetzgebung nicht die Gefahr, dass Beihilfe zum Suizid und Tötung auf Verlangen durchgeführt werden, obwohl die Möglichkeiten der Palliativmedizin noch nicht ausgeschöpft sind?

- das Problem der Freiwilligkeit: Kann ausgeschlossen werden, dass das Verlangen nach aktiver Sterbehilfe nicht von Dritten beeinflusst ist? Stellt die gesellschaftliche Akzeptanz der Beihilfe zum Suizid und der Tötung auf Verlangen nicht eine implizite Aufforderung für ein „sozialverträgliches Frühabsterben" dar?

- mögliche Missbrauchs- und Dammbruchgefahren: Welche - nicht beabsichtigten - gesellschaftlichen Entwicklungen setzen wir mit einer Legalisierung der aktiven Sterbehilfe in Gang? Besteht nicht die Gefahr, dass die Kriterien für eine erlaubte Tötung mit der Zeit ausgeweitet werden?

Es muss nicht sein, dass die genannten Gefahren tatsächlich eintreten. Entscheidend für die Debatte um eine gesetzliche Liberalisierung der Sterbehilfe ist jedoch die Frage, ob ihr Eintreten durch gesetzliche Bestimmungen (weitgehend) verhindern werden kann. Die Antwort darauf kann nicht von einer Person allein gegeben werden, sondern setzt eine gesellschaftliche Wertentscheidung voraus.

\section{Schlussbemerkung}

Die gesetzlichen Reglungen in den Niederlanden und Belgien verfolgen das Ziel, die Praxis der aktiven Sterbehilfe, die nachweislich auch in anderen Ländern existiert, transparenter zu gestalten, und die Lebensbeendigung auf Verlangen als letz- 
ten Ausweg für schwerstkranke Menschen zu ermöglichen, deren Leiden durch andere Maßnahmen nicht mehr zu lindern ist. Dieses Ziel ist - unabhängig davon, wie man persönlich dazu stehen mag - als Versuch, moralisch verantwortlich mit diesem Problem umzugehen, zu respektieren.

Die Praxis in beiden Ländern zeigt jedoch, dass dieses Ziel nur zum Teil erreicht wurde. Ein grundlegendes Problem besteht in der geringen Meldebereitschaft der Ärzte. Selbst in den Niederlanden, wo aktive Sterbehilfe seit gut 20 Jahren von den Gerichten geduldet praktiziert wird, wird nur etwa jede zweite Sterbehilfehandlung den Kontrollkommissionen gemeldet. Man darf annehmen, dass insbesondere jene Fälle, in denen die Sorgfaltskriterien nicht in vollem Umfang erfüllt sind, nicht gemeldet werden. Dies stellt die Funktion der Kontrollkommissionen und mir ihr die Effektivität der gesetzlichen Regelungen als solche erheblich in Frage. Ein weiteres Problem besteht in der schleichenden Ausweitung der „Indikation“ zur Sterbehilfe. Während aktive Sterbehilfe ursprünglich als Ultima-Ratio-Lösung für Patienten gesehen wurde, deren Leiden auf keine andere Weise mehr gelindert werden kann, zeigt die Praxis, dass die aktive Sterbehilfe im Falle ihrer gesetzlichen Zulassung nicht auf diese enge Patientengruppe beschränkt bleibt. Die gesellschaftliche Akzeptanz dieser Lösung führt vielmehr dazu, dass sie auch von anderen (Patienten-)Gruppen (z.B. psychisch Kranken, Dementen, Eltern für ihre behinderten Neugeborenen) eingefordert wird.

Ob diese Erfahrungen in Summe nun für oder gegen eine gesetzliche Zulassung der aktiven Sterbehilfe sprechen, kann und muss jeder für sich beurteilen. Ich persönlich respektiere den Weg, den die Niederländer und Belgier in dieser Frage eingeschlagen haben, lehne ihn aber für Deutschland ab. 


\section{„Terminale Sedierung“ - Ausweg im Einzelfall, Mittelweg oder schiefe Ebene?}

Christof Müller-Busch

\section{Im Schlafen sterben - ein Kriterium für ,gutes Sterben"?}

Die „Medikalisierung des Sterbens“ ${ }^{\text {“1 }}$ hat die Frage über die Verantwortung für den Sterbeprozess zu einem öffentlichen Thema gemacht, in dem über ein „gutes und in Würde Sterben" verstärkt reflektiert und diskutiert wird². Dabei ist zu berücksichtigen, dass die Vorstellungen gesunder Menschen über ein „gutes Sterben“ sich von denen, die sich als Sterbenskranke in Konfrontation mit dem Tode auseinandersetzen deutlich unterscheiden ${ }^{3}$. Ebenso finden sich im Vergleich zu Ge-

\footnotetext{
Illich I (1995): Die Nemesis der Medizin. Verlag CH Beck, München

2 Clark D (2002): Between hope and acceptance: the medicalisation of dying. BMJ, 324, 905-907

3 Proot IM, Abu-Saad HH, ter Meulen RH, Goldsteen M, Spreeuwenberg C \& Widdershoven GA (2004): The needs of terminally ill patients at home: directing one's life, health and things related to beloved others. Palliat Med, 18(1), 53-61
} 
sunden bei Angehörigen und Pflegenden, die Sterbende betreuen andere Vorstellungen, mit denen die Qualität des Sterbens beschrieben wird ${ }^{4}$.

Durch die Möglichkeiten der künstlichen Ernährung und Beatmung, der Aufrechterhaltung von Organfunktionen bis zum Ersatz lebenswichtiger Organe werden Zeitdauer und Art des Sterbens weitgehend von medizinischen Entscheidungen bestimmt. Übertherapie, Aktionismus oder nur symbolhaftes Handeln - aus welchem Grunde auch immer - ist besonders in Deutschland ein viel zu wenig problematisiertes, aber doch verbreitetes Phänomen. Hinzu kommt, dass zu den Kriterien, wann der Tod akzeptiert werden muss und Sterben ,zugelassen“ werden kann, unter betroffenen Patienten, Angehörigen und Ärzten häufig kein Konsens gesucht wird.

So ergeben sich durch die Möglichkeiten, den Todeszeitpunkt zu manipulieren, auch Interessenskonflikte, die das Sterben nicht mehr als autonome Leistung am Ende des Lebens erscheinen lassen, sondern als eine zunehmend von menschlich und moralisch Zumutbarem geprägte Ausnahmesituation. Unter diesen Bedingungen ist es auch schwieriger geworden, den Beginn des Sterbens unvoreingenommen zu erkennen und anzuerkennen. Ein „natürliches Sterben“ als autonome Leistung am Ende des Lebens oder infolge einer unheilbaren Erkrankung erscheint innerhalb des medizinischen Systems kaum noch möglich. Es findet als eine zunehmend von menschlich und moralisch Zumutbarem geprägte Ausnahmesituation statt, so dass Sterbsituationen für alle Beteiligten häufig auch als Dilemmasituationen empfunden werden. Dennoch wird unter den Bedingungen der modernen Medizin inzwischen auch verstärkt auf ein ,gutes Sterben“ bzw. den „guten Tod“ geachtet, so dass sich die Ermöglichung eines guten Sterbens auch als praktische medizinische Aufgabe stellt. Hospizbewegung und Palliativmedizin haben in den letzten Jahren wesentlich dazu beigetragen, dass sterbenskranken und sterbenden Menschen inzwischen mehr Aufmerksamkeit entgegengebracht wird. Auch wenn in der Umsetzung palliativer Konzepte noch erhebliche strukturelle Defizite bestehen, ist es ein wichtiges Anliegen der Palliativmedizin durch eine optimale Schmerz- und Symptomlinderung die letzte Lebensphase bzw. die Sterbesituation so erträglich und annehmbar zu machen, dass dadurch den zunehmenden Forderungen nach einer Legalisierung von Euthanasie oder assistiertem Suizid eine praktische Alternative entgegengesetzt wird. In diesem Zusammenhang hat auch die Möglichkeit einer gezielten Sedierung am Lebensende eine zunehmende Bedeutung bekommen. Durch die medikamentösen Möglichkeiten der Bewusstseinsdämpfung zur Leidensminderung aus den unterschiedlichsten Gründen ist auch die Frage aufgetaucht, ob eine Sedierung mit einer andauernden Bewusstseinsdämpfung oder -ausschaltung bis zum Tode nicht nur als Therapieoption bei anders nicht zu kontrollierenden Symptomen eingesetzt werden sollte,

4 Steinhauser KE, Clipp EC, McNeill M, Christakis NA, McIntyre LM \& Tulsky JA (2000): In search of a good death: Observations of patients, families, and providers. Ann Int Med, 132, 825-832 
sondern u. U. auch dann, wenn unter bestimmten Bedingungen ein ,in den Tod schlafen" gewünscht wird, so dass die ethische Bewertung einer Sedierung am Lebensende im Zusammenhang mit der Frage der Selbstbestimmung am Lebensende bzw. des Sterbezeitpunkts sehr kontrovers diskutiert wird. Vorzustellen sind aber auch Szenarien, in denen eine Sedierung auch in nicht terminalen Erkrankungssituationen, ja, sogar ohne Erkrankung als Möglichkeit einer wunscherfüllenden Medizin zur Anwendung gelangt ${ }^{5}$. Nicht auszuschließen ist zudem, dass die Möglichkeiten einer „terminalen Sedierung“ bis zum Tode auch von manchen als Ausweg angesehen werden, wenn bei unerträglichem Leid und aussichtsloser Prognose aktive Sterbehilfe erwogen wird.

Der weniger im Hinblick auf ihre Beschränkung auf terminale Krankheitsstadien aber evtl. in ihrer Intentionalität missverständliche Begriff „,terminale Sedierung" 6,7 wird in der kontrovers geführten Diskussion über Indikationen, Voraussetzungen, Formen und Ziele der Sedierung am Lebensende am häufigsten verwendet, auch wenn einige Experten den Begriff „palliative Sedierung“ oder palliative Sedierungstherapie zur Verdeutlichung der therapeutischen Intention bevorzugen 8, 9 . Eine einheitliche Terminologie konnte bisher nicht gefunden werden, was auch auf die unterschiedlichen ideologischen Standpunkte verweist, unter denen das Thema einer Sedierung am Lebensende behandelt wird.

Die Auseinandersetzung mit der Problematik Sedierung in terminalen Erkrankungssituationen begann für mich mit dem Prämedikationsgespräch bei einer 44jährigen Patientin, Ehefrau eines Rechtsanwalts, die eine Dauersedierung bis zum Tode forderte, falls die Durchführung der von ihr gewünschten palliativen Operation bei fortgeschrittenem Tumorleiden nicht möglich sei. Sie verlangte eindringlich, sie dann nicht mehr aus der Narkose aufwachen zu lassen. Die medizinischen, rechtlichen und weltanschaulichen Fragen, die in Konfrontation mit solchen Wünschen in Grenzsituationen entstehen, erfordern nicht nur Respekt vor den Werten anderer, sondern auch die Auseinandersetzung mit allen sich daraus ergebenden Problemen.

Auch in der Palliativsituation wird der Wunsch nach einem Dauerschlaf bis zum Tode, „im Schlaf zu sterben“, „nicht mehr aufzuwachen“ oder „eingeschläfert zu werden“, von Betroffenen und Angehörige nicht nur wegen unerträglicher Symptome, sondern auch aufgrund der Aussichtslosigkeit der Prognose und einer

$5 \quad$ Neitzke $G$ \& Frewer A (2004): Sedierung als Sterbehilfe? Zur medizinethischen Kultur am Lebensende. Ethik in der Medizin, 16, $323-333$

6 Byock IR (1994): When suffering persists. J Palliat Care, 10, 8-13

7 Enck RE (1991): Drug induced terminal sedation for symptom control. Am J Hosp Palliat Care, 8, 3-5

8 Morita T, Hirai K \& Okazaki Y (2002): Preferences for Palliative Sedation Therapy in the Japanese General Population. J Pall Med, 5, 375-386

9 Rousseau P (2001): Existential suffering and palliative sedation: A brief commentary with a proposal for clinical guidelines. Am J of Hosp and Pall Care, 18, 151-153 
sinnlosen Lebensperspektive in den letzten Jahren zunehmend häufiger geäußert ${ }^{10}$, so dass sich die Frage stellt, ob mit der sog. terminalen Sedierung nicht doch eine verdeckte Form der Euthanasie praktiziert wird, wenn in aussichtslosen Krankheitssituationen keine ausreichenden Maßnahmen zur Leidenslinderung und Unterstützungsmöglichkeiten gefunden werden und eine gezielte Lebensbeendigung aus rechtlichen, situativen und moralischen Erwägungen nicht durchgeführt werden kann.

Allerdings gibt es in der palliativmedizinischen Betreuung sterbenskranker Menschen auch einen breiten Konsens, dass im Bemühen um eine adäquate Symptomlinderung bei Patienten mit fortgeschrittenen Krebserkrankungen und anderen Erkrankungen im Endstadium auch der Einsatz von Sedativa in Extremsituationen angezeigt und notwendig ist. Trotz vieler Fortschritte in der Symptomkontrolle gilt ,terminale Sedierung“ auch in palliativmedizinischen Einrichtungen als wichtige therapeutische Option am Lebensende ${ }^{11}$, die zunehmend angewendet wird, so dass sich die Frage stellt, ob dies einem stärkeren Bedürfnis der Patienten oder einer veränderten Einstellung von Ärzten und Einrichtungen zur Frage einer guten Sterbegleitung zuzuschreiben ist. Bisher fehlen jedoch allgemein akzeptierte Richtlinien zu Indikation, Technik und Dokumentation einer Sedierung im Rahmen der palliativen Betreuung am Lebensende, so dass es sicherlich notwendig ist Stellenwert und Grenzen dieser Therapieoption zu verdeutlichen und durch Transparenz Fehlentwicklungen zu vermeiden.

\section{Medizinische Probleme und Konflikte in Grenzsituationen}

Trotz der von der Bundesärztekammer im Jahre 2004 überarbeiteten Grundsätze zur ärztlichen Sterbebegleitung ${ }^{12}$ und der Handreichungen für Ärzte zum Umgang mit Patientenverfügungen ${ }^{13}$ bestehen bei vielen Ärzten fachliche und ethische Unsicherheiten um die Grenzen ärztlicher Behandlungspflicht und die Bedeutung des Patientenwillens in konkreten Entscheidungssituationen. Hinzu kommen Bedenken und Wissensdefizite, wie, wann und in welchem Rahmen „palliative“ Maßnahmen am Ende des Lebens durchgeführt werden können. Die Problembereiche, mit denen sich Ärzte in der Vorphase des Todes auseinandersetzen müssen, spielen in der ärztlichen Aus- und Weiterbildung so gut wie keine Rolle. Sie

\footnotetext{
10 Müller-Busch HC, Jehser T \& Andres I (2003): Sedation in palliative care - a critical analysis of 7 years experience. BMC Palliat Care, 2, 2

11 Müller-Busch HC (2006): Palliative Sedierung am Lebensende. In : Deutsche Krebsgesellschaft (Hrsg.): Kurzgefasste Interdisziplinäre Leitlinien 2006. Zukschwerdt Verlag, München, S. 622-624

12 Bundesär:tekammer (2004): Grundsätze der Bundesärztekammer zur ärztlichen Sterbebegleitung. Dtsch Arztebl, 101, 1075-1077

13 Bundesärrtekammer (1999): Handreichungen für Ärzte zum Umgang mit Patientenverfügungen. Dtsch Arztebl, 96, 2720-2721
} 
lassen sich folgendermaßen charakterisieren: 1. Beurteilung der Prognose, 2. Umgang mit Selbstbestimmung, 3. Verzichtsentscheidungen, 4. Sterbebegleitung und 5. Umgang mit Ressourcen. Mangelnde Bereitschaft, diese Problembereiche bei Entscheidungen am Ende des Lebens zu berücksichtigen, kann, ja sie muss zu Konflikten führen, wenn in der Betreuung sterbenskranker Menschen unterschiedliche Meinungen über Wege und Zielvorstellungen in der letzten Lebensphase aufeinander treffen ${ }^{14}$ und die Grundprinzipien, die das Verhalten und Handeln in solchen Grenzsituationen bestimmen sollen, nicht gekannt bzw. beachtet werden.

Probleme und Konflikte in Grenzsituationen zwischen Leben und Tod haben in der Regel drei Dimensionen: eine philosophisch-religiöse, durch die allgemein anerkannte ethisch-moralische Grundsätze, aber auch unterschiedliche Erkenntniswege und Sichtweisen zum Ausdruck kommen, eine rechtliche, durch die gesellschaftliche und wirtschaftliche Rahmenbedingungen berücksichtigt werden und die im Einzelfall einen Schutz gewährleisten soll, sowie eine medizinische, in denen sich die prognostischen Kriterien sowie therapeutische Möglichkeiten und Erfahrungen für konkrete Behandlungssituationen manifestieren.

\section{Sedierung am Lebensende - eine palliativmedizinische Therapieoption}

Das Grundanliegen der Palliativmedizin ist es - wie es die Pioniere der modernen Palliativmedizin und Hospizidee Derek Doyle und Cicely Saunders einmal formuliert haben -, dem Leben bei fortgeschrittenen Erkrankungen nicht mehr Zeit, sondern der verbleibenden Zeit mehr Leben zu geben ${ }^{15,16}$ und das Sterben nicht hinauszuziehen ${ }^{17}$. Palliativmedizin konzentriert sich auf Situationen, in denen eine Erkrankung nicht mehr heilbar, irreversibel ist. Allerdings geht es in der Palliativmedizin nicht nur darum, mit der besonderen fachlichen Kompetenz einer neuen Spezialdisziplin, Maßnahmen zur Linderung belastender Symptome, z. B. von Schmerzen durchzuführen, so dass trotz fortgeschrittenen Erkrankungsstadiums eine optimale Lebensqualität für die Restzeit des Lebens erzielt wird, sondern auch durch psychosoziale Begleitung und evtl. spirituellen Beistand die existentiellen Fragen, die sterbenskranke Menschen beschäftigen, aufzunehmen und sich mit

14 Müller-Busch HC, Klaschik. E, Oduncu FS, Schindler T \& Woskanjan S (2003): Euthanasie bei unerträglichem Leid ? Eine Studie der Deutschen Gesellschaft für Palliativmedizin zum Thema Sterbehilfe im Jahre 2002. Z Palliativmed, 4, 75-84

15 Doyle D, Hanks GWC \& MacDonald N (eds.) (1993): Oxford Textbook of Palliative Medicine. Oxford Univ Press, New York

16 Patt RB (1996): Put life into their days, not just days into their life. Am J of Hospice \& Palliative Care, 13(5), 13-18

17 Saunders C (1978): The philosophy of terminal care. In: Saunders C (ed.): The management of terminal disease. Edward Arnold, London 
ihnen auseinander zu setzen. Ein wichtiges Anliegen einer umfassenden palliativen Betreuung ist es auch, Orientierungen $\mathrm{zu}$ finden, wenn das Leben aussichtslos erscheint und Perspektiven zu entwickeln, wenn scheinbar keine Hoffnung mehr besteht. Gezielte Lebensverkürzung gehört nicht in den Verantwortungsbereich der Palliativmedizin. Die Möglichkeiten und ethischen Prinzipien der Palliativmedizin werden besonders in Deutschland als angemessene Antwort auf die Forderungen nach einer gesetzlichen Regelung der Euthanasie wie in den Niederlanden oder Belgien angesehen ${ }^{18}$, so dass die Förderung der Palliativmedizin eine wichtige politische Aufgabe in der Auseinandersetzung mit der sog. aktiven Sterbehilfe darstellt.

\section{Ethische Aspekte und Autonomie}

Die Europäische Gesellschaft für Palliative Care (EAPC) weist in ihrem Positionspapier zur Euthanasie und ärztlich assistiertem Suizid darauf hin, dass „terminale“ oder „palliative“ Sedierung von der Euthanasie deutlich unterschieden werden muss. Bei der Euthanasie ist es die Intention, den Patienten zu töten, das Vorgehen besteht darin, ein tödliches Medikament zu verabreichen und das erfolgreiche Ergebnis ist der sofortige Tod. Die Intention von terminaler Sedierung ist es, unerträgliches Leiden zu lindern; das Vorgehen besteht darin, ein sedierendes Medikament zur Symptomkontrolle einzusetzen und der Erfolg dieser Maßnahme ist die Linderung der belastenden Symptome. In der Palliativmedizin kann eine milde Sedierung therapeutisch eingesetzt werden, die den Wachheitszustand des Patienten oder seine Kommunikationsfähigkeit nicht wesentlich beeinträchtigt. In seltenen Fällen kann das klar formulierte therapeutische Ziel nur durch eine tiefe Sedierung erreicht werden, mit der Folge, dass die Bewusstseinslage des Patienten stark eingeschränkt wird. In solchen Fällen muss die Tiefe der Sedierung regelmäBig neu überdacht werden. Zudem werden sedierte Patienten kontinuierlich überwacht und künstlich ernährt oder hydriert, falls dies im Krankheitsverlauf indiziert sein sollte. Generell ist eine tiefe Sedierung immer nur für einen bestimmten Zeitraum vorgesehen ${ }^{19}$. Diese Definition der „terminalen Sedierung“ verdeutlicht, dass es sich um eine situativ steuer- und kontrollierbare Therapieoption zur Symptomkontrolle und nicht um ein den Tod des Patienten intendierendes Verfahren handelt.

Die Ermöglichung und Begleitung eines - sicherlich nicht immer leichten und einfachen - autonomen Sterbens als Vollendung einer autonomen Lebensgestaltung, benötigt umfassende fürsorgliche Hilfe in der Sterbesituation, die von Re-

18 Klaschike E, Nauck F, Ostgathe C \& Hoffmann-Menzel H (2002): Palliativmedizin - eine ärztliche Verpflichtung. Anaesthesiol Reanim, 27, 68-74

19 Materstvedt LJ, Clark D, Ellershaw J, Forde R, Boeck. Gravgaard AM, Müller-Busch HC, Porta I, Sales J \& Rapin CH (2003): Euthanasia and physician-assisted suicide: a view from an EAPC Ethics Task Force. Pall Med, 17, 97-101 
spekt aber auch empathischer Verantwortung geprägt sein sollte, wobei die gezielte Herbeiführung des Todes, z.B. durch Euthanasie, eigentlich dem Autonomieprinzip widerspricht, da sie Autonomie zerstört und nicht fördert. Deshalb sollten in der medizinethischen Diskussion um den Stellenwert einer Sedierung am Lebensende die von Beauchamp und Childress entwickelten Prinzipien, Autonomie, Benefizienz, Non-Malefizienz und Gerechtigkeit ${ }^{20}$, stärker in ihrem Spannungsverhältnis zueinander und weniger in der von der Autonomie dominierten starren hierarchischen Zuordnung Berücksichtigung finden - im Einzelfall sicherlich mit unterschiedlicher Gewichtung.

\section{Sedierung am Lebensende - Definition - Formen - Grundlagen}

Viele der in der Palliativmedizin eingesetzten Medikamente haben meist temporär und bei hohen Dosen auftretende, sedierende Nebenwirkungen. In der Regel sind diese auch als sekundäre Effekte bezeichneten Nebenwirkungen unerwünscht. Gelegentlich ist jedoch eine Benommenheit oder Sedierung als primärer Effekt erwünscht, z.B. bei extremer Unruhe bzw. in Situationen, in denen die Symptome anderweitig nicht kontrolliert werden können. Bei der primär intendierten Sedierung können verschiedene Formen der Bewusstseinsdämpfung unterschieden werden: oberflächlich bis tief, mit schnellem oder allmählichem Wirkungseintritt, intermittierend oder kontinuierlich ${ }^{21}$. Neben Häufigkeit und Indikation werden auch Form und u. U. damit verbundene Nebeneffekte einer Sedierung am Lebensende in einem hohen Maße von konzeptionellen Einstellungen, professioneller Erfahrung in Symptomkontrolle, ambulanten oder stationären medizinischen Versorgungsstrukturen sowie kulturellen und religiösen Haltungen bestimmt ${ }^{22}$.

Die von einer internationalen Consensus Group erarbeitete Definition der palliativen oder terminalen Sedierung: „Einsatz von Medikamenten (in der Regel Benzodiazepine +/- Opioide iv. oder sc.) zur Symptomkontrolle in der Terminalphase mit der Absicht, das Bewusstsein so weit zu dämpfen, dass bis zum Tode weitgehend Beschwerdefreiheit besteht.“. Bewusstseinsdämpfung ist die Methode, das gewünschte Ziel: die Beschwerdelinderung ${ }^{23}$.

20 Beauchamp TL \& Childress JF (1994): Principles of biomedical ethics. 3rd Edition. Oxford Univ Press, New York

21 Morita T, Tsunoda J, Inoue S, et al. (2000): Terminal sedation for existential distress. Am J Hospice Palliat Care, 17, 189-195

22 Radbruch L (2002): Reflections on the use of sedation in terminal care. EJPC, 9, 237-239

23 Die demnächst erscheinende Übersichtsarbeit der Konsensusgruppe: De Graeff A \& Dean M (2006):

Palliative Sedation Therapy in the last weeks of life - a literature review and recommendations for standards. J Palliat Med, in publ. 
Einigkeit besteht darüber, dass der Zeitpunkt des Einsatzes einer terminalen Sedierung auf terminale oder finale Krankheitsstadien beschränkt werden sollte ${ }^{24}$. Über den Zeitpunkt, in welchem Stadium einer Erkrankung eine solche Maßnahme intermittierend oder mit bis zum Tode andauernder kontinuierlicher Bewusstseinsdämpfung indiziert ist und ob eine Ernährungs- oder Flüssigkeitstherapie fortgesetzt werden soll, gehen die Meinungen auseinander ${ }^{25}$. Besonders die Indikation zur Flüssigkeitstherapie in der terminalen Krankheitsphase wird mehr von kulturell, institutionell und religiös bestimmten Überzeugungen als von einer klinischen Evidenz über den Nutzen einer solchen Behandlung bestimmt, so dass Ängste und Präferenzen der Betroffenen und Angehörigen sorgfältig berücksichtigt werden sollten. Hinzu kommen häufig auch ethische und professionell normative Grundsätze zur Ernährungs- und Flüssigkeitstherapie in der Endphase des Lebens $^{26}$.

Die Einwilligung des Patienten und seiner Angehörigen wird als wichtige und notwendige Voraussetzung einer Sedierung am Lebensende angesehen. Besonders bei Patienten mit Erkrankungen, bei denen in der Endphase u. U. schwer zu kontrollierende oder mit starken Ängsten verbundene Symptome erwartet werden können, sollte die Möglichkeit einer Sedierung in der Sterbephase frühzeitig und mit allen Beteiligten erörtert werden. Dennoch kann in Notsituationen z.B. im Finalstadium einer Erkrankung mit massiven Blutungen, extremer Unruhe und Erstickungsangst eine Sedierung zur Beruhigung der Sterbephase auch ohne explizite Einwilligung des Betroffenen angezeigt sein. Im Interesse einer nachvollziehbaren Indikation sollte die Entscheidung allerdings besonders sorgfältig dokumentiert werden und transparent sein. Unter Berücksichtigung der prognostischen Unsicherheit einer Vorhersagbarkeit der Grenzlinie zwischen Leben und Tod sowie der besonders bei fortgeschrittenen „unheilbaren“ Erkrankungen von medizinischen Entscheidungen und Maßnahmen bestimmten Verläufe, berührt die Frage des Einsatzes von Sedativa am Lebensende auch die Frage, ob dadurch möglicherweise eine Lebensverkürzung bewirkt wird, die - wenn sie als unbeabsichtigte Nebenfolge der Sedierung auftritt - unter der Doktrin des Doppeleffektes bzw. als eine Form der sog. indirekten Sterbehilfe bewertet würde ${ }^{27}$.

Es soll in diesem Zusammenhang nochmals betont werden, dass das Ziel der Sedierung nicht notwendigerweise die Herbeiführung des Tiefschlafs oder eines komatösen Zustandes bis zum Tode ist, sondern eine optimale Symptom- und Angstminderung durch eine angemessene Dosierung der Sedativa und Dauer der

24 Cherny N, Coyle N \& Foley K (1996): Guidelines in the care of the dying cancer patient. Hematol Oncol Clin North Am, 1, 261-286

25 Jansen LA \& Sulmasy DP (2002): Sedation, alimentation, hydration, and equivocation: careful conversation about care at the end of life. Ann Intern Med, 136, 845-849

26 Viola RA, Wells GA \& Petersen J (1997): The effects of fluid status and fluid therapy on the dying: a systemic review. J of Palliat Care, 13, 41-52

27 Boyle J (2004): Medical ethics and double effect: the case of terminal sedation. Theor Med Bioeth, 25, 51-60 
Sedierung. Die Kombination mit Opiaten und anderen Medikamenten sollte die angestrebten Behandlungsziele berücksichtigen, sofern diese für eine gute Symptomkontrolle in der Terminalphase indiziert sind. Die Sedierungstiefe kann durch eine intermittierende und situativ angepasste Dosistitrierung an den Schlaf-WachBedarf des Patienten adaptiert werden und am Tag eine entspannte (eingeschränkte) Kommunikation erlauben. Eine milde Sedierung schließt eine orale Nahrungsund Flüssigkeitsaufnahme nicht aus. In einer eigenen Untersuchung konnten 2/3 der sedierten Patienten noch Flüssigkeit und $13 \%$ Nahrung zu sich nehmen, bei 1/3 der Patienten erfolgte die intravenöse Flüssigkeitszufuhr im Verlauf der Sedierung nach klinischen Zeichen von Durst ${ }^{28}$. Sedierung am Lebensende kann und darf personelle Betreuung, Nähe und mitfühlendes Verständnis nicht ersetzen. Die sorgfältige Kontrolle und Dokumentation der Sedierungstiefe und -dauer sowie vitaler Parameter erhöht nicht nur die Transparenz dieser wichtigen medizinischen Behandlungsmöglichkeit in der Sterbebegleitung, sondern sollte auch die Aufmerksamkeit für die besonderen Bedürfnisse Sterbender fördern und den Angehörigen die Angst und das Leiden in einer auch für sie oft „unerträglichen“ Abschiedssituation erleichtern.

\section{Sedierung am Lebensende - Gefahren, Indikationen und Risiken}

Die Möglichkeit des Missbrauchs einer Sedierung bei sterbenskranken und sterbenden Menschen ist besonders durch Medienberichte aus den Niederlanden aber auch in Deutschland ins Blickfeld geraten, nachdem „,terminale Sedierung“ mit dem Ziel der Lebensbeendigung in zunehmender Häufigkeit bei schwerstkranken Patienten eingesetzt wurde, um den strengen Formalien der dortigen Euthanasiegenehmigungsverfahren zu entgehen ${ }^{29}, 30$. So wurde in einer repräsentativen Umfrage in den Niederlanden festgestellt, dass fast die Hälfte der Ärzte bereits einmal eine „terminale Sedierung” durchgeführt hatte. In jedem sechsten Fall geschah dies in der expliziten Absicht, den Tod des Patienten herbeizuführen ${ }^{31}$.

Die Frage, welche Bedingungen und Symptome eine Bewusstseinsdämpfung bzw. -ausschaltung bis zum Eintritt des Todes rechtfertigen bzw. ob das Sterben durch eine medikamentöse Sedierung oder sogar Narkose anästhesiologisch begleitet werden soll und darf, wird aus palliativmedizinischer Sicht kontrovers dis-

\footnotetext{
28 Rousseau P (2001), vgl. Anm. 9

29 Kolfschooten v F (2003): Dutch television report stirs up euthanasia controversy. Lancet, 361, 1352-1353

30 Dablkamp J, Fröblingsdorf $M$ \& Ludwig U (2003): Tiefschlaf bis zum Tod. Der Spiegel, 41, 206-208

31 Rietjens $J A C$, van der Heide A, V rakeking AM, Onwuteaka-Philipsen BD, van der Maas PJ \& van der Wal G (2004): Physician Reports of Terminal Sedation without Hydration or Nutrition for Patients Nearing Death in the Netherlands. Ann of Int Med, 141, 178-185
} 
kutiert $^{32}$. Während in der Intensivmedizin ein weitgehender Konsensus besteht, dass eine Sedierung auch in der Sterbesituation fortgesetzt wird ${ }^{33}$, wird von Kritikern der palliativen oder terminalen Sedierung befürchtet, dass für Abschied und Sinnfragen, die Menschen in der Begegnung mit Sterben und Tod immer wieder bewegen, durch technische Lösungen immer weniger Raum bleibt ${ }^{34}$ und sich durch die Medikalisierung des Sterbens auch die Elemente und Vorstellungen eines guten Todes verändern.

Persistierende, therapieresistente Symptome werden in den meisten Übersichten als Hauptindikationen für eine Sedierung in terminalen Krankheitsstadien genannt. Dazu gehören therapieresistentes agitiertes Delir (39\%), Atemnot (38 $\%$ ), Schmerz (22\%), Angst und Stress (21\%), akute Blutungen (9\%) sowie Übelkeit und Erbrechen $(6 \%)^{35}$. In einer in Großbritannien durchgeführten Umfrage wurden von 61 Palliativmedizinern bei über $50 \%$ der sedierten Patienten mehrere Symptome als Grund genannt ${ }^{36}$. Auch wenn in verschiedenen Übersichten ,existentielles Leid“ als wichtige Indikation zur „terminalen Sedierung“ genannt wird, ist es im Vergleich zu den mehr körperlichen Symptomen noch schwieriger Häufigkeitsangaben zu psychosozialen Belastungsfaktoren als Indikation für eine Sedierung zu finden, zumal die Bereitschaft eine Sedierung am Ende des Lebens in emotionalen und psychosozialen Stresssituationen in Erwägung zu ziehen, stark von strukturellen Voraussetzungen bzw. institutionellen und kulturellen Einstellungen im Umgang mit der letzten Lebensphase bestimmt wird ${ }^{37,38}$. In verschiedenen retrospektiven und prospektiven Untersuchungen wurde die Häufigkeit einer Sedierung in der Terminalphase bei Patienten mit unheilbaren Erkrankungen zwischen 8 und $57 \%$ der verstorbenen Patienten angegeben ${ }^{39}$.

Während z.B. in einer anonymen norwegischen Untersuchung nur $22 \%$ der antwortenden Ärzte eine Sedierung bei Sterbenden durchgeführt hatten und therapieresistente Schmerzen als Hauptgrund angegeben wurden ${ }^{40}$, kommt eine palli-

32 Tännsiö T (2000): Terminal sedation - a possible compromise in the euthanasia debate? Bull Med Eth, $163,13-22$

33 Hawryluck LA, Harvey WRC, Lemieux-Charles L \& Singer PA (2002): Consensus guidelines on analgesia and sedation in dying intensive care unit patients. BMC Medical Ethics, 3, 3,

http://www.biomedcentral.com/1472-6939/3/3 (Stand: 26.09.2006)

34 Craig G (2002): Terminal sedation. CMQ, www.catholicdoctors.org.uk/CMQ/

Feb_2002/terminal_sedation.htm (Stand: 26.09.2006)

35 de Graeff A, Strasser F: Indications and conditions for palliative sedation. International Consensus Group on Terminal Sedation - Diskussionspapier (unveröffentlicht), http://www.eapcnet.org/forum/ (Stand: 26.09.2006)

36 Chater S, Viola R, Paterson J, et al. (1998): Sedation for intractable distress in the dying - a survey of experts. Palliat Med, 12, 55-269

37 Cowan JD \& Walsh D (2001): Terminal sedation in palliative medicine-definition and re-view of the literature. Support Care Cancer, 9, 403-407

38 Porta Sales J (2001): Sedation and terminal care. Eur J Palliat Care, 8, 97-100

39 Müller-Busch HC (2004): Sterbende sedieren? DMW, 129, 701-704

40 Forde R, Olaf G, Aasland OG, Falkum E, Brewik H \& Kaasa S (2001): Palliative sedation to the dying patient. Tidsskr Nor Lægeforen, 121, 1085-1088 
ative oder terminale Sedierung nach Untersuchungen in Spanien und Italien in bis zu $57 \%$ der Sterbenden zum Einsatz ${ }^{41}$. Eine eigene Untersuchung über den Einsatz von sedierenden Medikamenten in den letzten 48 Stunden des Lebens zeigte eine Gesamtinzidenz von 14,5 \% in den Jahren 1995 - 2002 mit zunehmender Häufigkeit in den letzten drei Jahren. Gleichzeitig zeigte sich, dass sich der zunächst mehr bei „körperlichen“ Symptomen erfolgte Einsatz von Sedativa in den letzten Jahren zugunsten von mehr psychosozialen Indikationen verschoben hatte, während z.B. Schmerz insgesamt nur in 2,5 \% für die Entscheidung zur Sedierung ausschlaggebend war ${ }^{42}$.

Die Möglichkeit, durch eine Sedierung bei schwerstkranken Patienten schwerste therapierefraktäre Leidenszustände effektiv zu lindern, diese Therapieoption aber unter Umständen zu missbrauchen, um den Todeseintritt medizinisch zu beschleunigen, hat die Notwendigkeit klarer ethischer Kriterien verdeutlicht ${ }^{43}$. Die Respektierung und u. U. Förderung von Autonomie ist ein wesentliches Element der Palliativbetreuung, so dass auch im Zusammenhang mit der Frage der sog. „terminalen Sedierung“ die Berücksichtigung des Autonomieprinzips von zentraler Bedeutung ist. Autonomes Sterben ist nicht nur ein moralisches Recht, sondern auch eine jedem Menschen zugehörige Fähigkeit ${ }^{44}$. In sterbenahen Situationen angemessene und willensorientierte Entscheidungen zu finden und das Handeln an ethischen Prinzipien zu orientieren bedeutet auch, die professionell fachbestimmte Herangehensweise an die komplexen Fragen am Lebensende zurückzustellen zugunsten einer Haltung, die auch in schwierigen Situationen von der Bereitschaft geprägt ist, ,gemeinsame Verantwortungspartnerschaft" für das Sterben und dessen Qualität zu übernehmen und nicht diese Verantwortung einseitig zu verlagern. Nicht Autonomie in Konfrontation mit Paternalismus ist das Leitprinzip, das der Patient einklagt, sondern Respekt, Redlichkeit, Solidarität und Mitgefühl. Die Szenarien in der Nähe des Todes benötigen nicht Menschenwürde durch mehr Selbstbestimmung, sondern Menschenwürde in Schwäche ${ }^{45}$.

\section{Zusammenfassung}

Sedierung am Lebensende ist eine effektive medizinische Therapieoption in Extremsituationen „unerträglichen Leids“. Die Indikation zum Einsatz wird in be-

\footnotetext{
41 Ojeda Martin M, Navarro Marrero A \& Gomez Sancho M (1997): Sedacion y enfermoa on-cologico terminal. Med Pal, 4, 101-108

42 Rousseau P (2001), vgl. Anm. 9

43 Materstvedt LJ \& Kaasa S (2001): The ethics of terminal sedation. Poster $7^{\text {th }}$ Congress of EAPC. Palermo 2001, http://www.medisin.ntnu.no/ims/kreft/slb/Dokument/Abstract/ materstvedt_og_kaasa_Palermo_29_mars.pdf (Stand: 13.02.2004)

44 Woods S (2002): Respect for autonomy and palliative care. In: Ten Have H, Clarke D (eds.): The ethics of palliative Care - European Perspectives. Open University Press, Buckingham

45 Sass HM \& Kielstein R (2003): Die Patientenverfügung in der Praxis. Lit Verlag, Münster
} 
sonderer Weise auch von den Wertvorstellungen der Beteiligten bestimmt. Die Sedierungstiefe und -art sollte individuellen Bedürfnissen angepasst werden, die Durchführung erfordert Aufklärung aller Beteiligten und ,informierte Zustimmung“. Die Möglichkeit des Missbrauchs einer Sedierung zur Beschleunigung des Sterbens erfordert nicht nur fachliches Wissen über Indikation, Art und Voraussetzungen ihres Einsatzes, sondern auch hohe ethische Kompetenz und Einfühlungsvermögen in die Bedürfnisse und Nöte sterbenskranker Menschen. Frühzeitige Aufklärung, Kommunikation über Ziele und Absichten einer terminalen Sedierung, Richtlinien zur Durchführung, Dokumentation und Transparenz sind notwendig und sollten dazu beitragen, den Stellenwert der Sedierung im Rahmen der palliativen Betreuung am Lebensende zu verdeutlichen. Die durch eine medikamentöse Bewusstseinsdämpfung intendierte Symptomlinderung sollte von einer gezielten Beschleunigung des Todes deutlich unterschieden werden - auch wenn mit dem Einsatz von Sedativa eine unbeabsichtigte Lebenszeitverkürzung verbunden sein kann, jedoch nicht notwendigerweise sein muss. Nicht die Sedierung soll zum Tode führen, sondern die zugrunde liegende Erkrankung. Die erkenntnismethodisch und philosophisch interessante Frage, unter welchen Voraussetzungen eine künstliche Bewusstseinsdämpfung am Ende des Lebens auch als Qualitätsmerkmal eines guten Sterbens angesehen werden kann, sollte weiter diskutiert werden. 


\section{Menschenwürdig sterben. Die Diskussion um die Sterbehilfe aus der Sicht der evangelischen Kirche und der evangelischen Ethik}

Reiner Anselm

\section{1. Überscharfe Reaktionen und falsche Alternativen}

Als die Schweizer Organisation „Dignitas“ im Herbst 2005 in Hannover ein Büro eröffnete, ließ die Reaktion der evangelischen Landesbischöfin nicht lange auf sich warten: Eine gewerbsmäßig organisierte Praxis der Beihilfe zum Suizid widerspreche zutiefst christlichen Grundsätzen, sie sei moralisch verwerflich und solle darum auch gesetzlich verboten werden. Die Auseinandersetzung um „Dignitas“, verbunden mit der Forderung des damaligen Hamburger Justizministers Roger Kusch, zu diesem Zeitpunkt immerhin noch Mitglied der CDU, nach einer Liberalisierung der Sterbehilfe ${ }^{1}$ nahmen die Kirchen zum Anlass, mit Nachdruck ihre Position zu diesem Themenkreis in der Öffentlichkeit zu vertreten und sich dabei als Vertreterinnen eines umfassenden Lebensschutzes zu präsentieren: So hatte etwa die Hamburger Bischöfin Maria Jepsen - ebenfalls im Hamburger Abendblatt - kurz zuvor konstatiert: „Als Kirche vertreten wir die Auffassung, dass man

1 Roger Kusch: Leben und Sterben in Würde. Hamburger Abendblatt vom 11.10.2005 
sich nicht das Leben nehmen darf. Das wäre gegen Gottes Willen.“ Dementsprechend unterscheiden sich die vielfältigen kirchlichen Reaktionen und Verlautbarungen mitunter im Ton, nicht aber in der Sache: Man setzt sich vehement für eine Verbesserung der palliativmedizinischen Versorgung ein, lehnt aber jede Form der aktiven Sterbehilfe ab. Die Linderung individuellen Leids wird ausdrücklich gefordert, jedes Eingreifen aber abgelehnt, das dazu geeignet scheint, den Charakter des Todes als eines Geschicks zu negieren. So heißt es in der jüngsten Stellungnahme zu dieser Problematik, einer Entschließung der Landessynode der evangelisch-lutherischen Kirche in Bayern, die sich dabei eine Resolution des Diakonischen Werks Bayern zu Eigen machte: „Für die Diakonie liegt menschliches Leben von seinem Beginn bis zu seinem Ende in der Hand Gottes, der ihm Würde und Sinn verleiht. Es widerspricht darum unserem Glauben, das Sterben durch aktives Eingreifen vorzeitig zu beenden. Wir treten dafür ein, dass Menschen in Krankheit und Sterben begleitet und ihre Schmerzen gelindert werden. “2 Und in der im letzten Frühjahr verabschiedeten Stellungnahme der Kammer für öffentliche Verantwortung der EKD heißt es analog: „Nach christlichem Verständnis darf der Tod eines Menschen nicht herbeigeführt, sondern muss abgewartet werden. Denn Christen sehen sich selbst als Geschöpfe Gottes. Geburt und Tod liegen somit in Gottes Hand, der Tod wird (ebenso wie die Geburt) als ein Geschick verstanden.“3

So eindeutig diese Positionsbestimmung wirkt, so große Probleme wirft sie doch auch auf. Dabei soll hier unerörtert bleiben, welche Funktion solche religiös fundierten Argumente in einem politischen Diskurs beanspruchen können ${ }^{4}$. Aus theologischer Perspektive ist vielmehr die grundsätzlichere Frage von Interesse, welche Geltung solche Aussagen vor dem Hintergrund der christlichen Tradition beanspruchen können. Dabei ist zunächst festzuhalten, dass die apodiktische Formel, dass der Tod eines Menschen auf keinen Fall herbeigeführt werden kann, zumindest ein Novum in der christlich-ethischen Theoriebildung darstellt, für das gesonderte Gründe angeführt werden müssten. Zwar kennen Bibel und christlicher Glaube, ebenso wie die meisten religiösen Traditionen, einen besonderen Wert des menschlichen Lebens. Doch ein solcher absoluter Lebensschutz, wie er in der jüngsten Erklärung der EKD proklamiert wird, kann sich weder auf das Schriftzeugnis noch auf die Tradition berufen: Die biblischen Texte sind hier ebenso uneindeutig wie die überkommenen ethischen Positionsbestimmungen in

2 Zit. nach Amtsblatt für die Ev.-luth. Kirche in Bayern Nr. 1, 1.1.2006, 2

3 Sterben hat seine Zeit. Überlegungen zum Umgang mit Patientenverfügungen aus evangelischer Sicht. Ein Beitrag der Kammer für Offentliche Verantwortung der Evangelischen Kirche in Deutschland, Hannover 2005, 12

4 Auf diese Problematik der EKD-Stellungnahme Sterben hat seine Zeit hat bereits Klaus-Michael Kodalle hingewiesen: Ders. (2005): Die Stellungnahme der Evangelischen Kirche zum Stellenwert der Patientenverfügung. Kritische Anmerkungen. ZEE, 49, 223-229; weiter gehend zu dieser gerade auch in den USA im Anschluss an John Rawls Schriften zum politischen Liberalismus intensiv geführten Debatte vgl. Jürgen Habermas (2005): Zwischen Naturalismus und Religion. Philosophische Aufsätze. Frankfurt/M., bes. S. $127 \mathrm{ff}$. 
Theologie und Kirche. Denn zumindest der Beruf des Soldaten wurde und wird als durchaus vereinbar mit dem christlichen Glauben angesehen, und auch im Blick auf die Todesstrafe wurde deren Zulässigkeit lange Zeit selbstverständlich konstatiert. Auch die Beurteilung des Selbstmords ist schon in der biblischen Tradition keineswegs eindeutig (vgl. 2 Sam 19,1; 2 Kön 16,18; Mt 27,5), und auch in der ethischen Theoriebildung der Christentumsgeschichte ist die Einschätzung dazu ebenso kontrovers wie in der Philosophie.

Der theologische Grund für diesen keineswegs so absoluten Lebensschutz dürfte darin liegen, dass die christliche Urteilsbildung dem irdischen Leben nur einen vorläufigen Status beigemessen hat. Gerade die Vorstellung vom Menschen als Geschöpf Gottes bringt ja auch zum Ausdruck, dass der Mensch als Geschöpf Gottes eben nicht mit diesem identisch ist. Leben ist darum endlich und eben nicht göttlich. Umgriffen wird diese Vorstellung von der Endlichkeit des Lebens freilich von der Vorstellung von der Auferstehung der Toten und dem ewigen Leben. Aber gerade aus der Gegenüberstellung von ewigem und irdischem Leben ergibt sich eine differenzierte Sicht des irdischen Lebens, dessen Wert keineswegs so absolut gesehen wurde und auch nicht so absolut zu sehen ist, wie das in manchen kirchlichen Stellungnahmen zur Sterbehilfe derzeit den Eindruck macht. Für den christlichen Glauben ist vielmehr die Unterscheidung zentral zwischen diesem und dem künftigen Leben, zwischen irdischem und ewigem Leben.

Insbesondere das Johannesevangelium gruppiert seine Schilderung des Auftretens Jesu um diese Vorstellung vom wahren Leben, das in der Person Jesu Christi aufscheint und doch in unübersehbarem Kontrast steht $\mathrm{zu}$ allen Vorstellungen eines bloß irdischen Lebens. Schon im Prolog wird deutlich, dass Jesus selbst das Leben ist, eine Aussage die dann in 14,6 in der Aussage gipfelt: Ich bin der Weg, die Wabrheit und das Leben (vgl. Joh 11,25). Diejenigen, die um ihn sind, missverstehen dies allerdings ebenso gründlich wie konsequent: Für sie ist Leben immer nur etwas diesseitiges, und darum können etwa Nikodemus (Joh 3, 1-21) oder die Samaritanerin (Joh 4) Jesu Kommen und das in ihm aufscheinende zukünftige Leben überhaupt nicht richtig deuten.

Es ist nicht ohne Ironie, vielleicht sogar nicht ohne Tragik, dass die Kirchen sich in den vergangenen Jahren selbst auf die Seite derer geschlagen haben, die das Leben nur mehr aus seiner diesseitigen Perspektive wahrnehmen möchten - und damit gegenwärtig möglicherweise auch einer Selbstsäkularisierung des Christentums Vorschub leisten. Denn wo von einem unbedingten Wert irdischen Lebens die Rede ist, verliert die christliche Hoffnung nach demjenigen Leben, das unsere Erfahrung und damit auch unsere Sterblichkeit überschreitet und umgreift, seinen Rückhalt.

Um hier nicht falsch verstanden zu werden: der Verweis auf ein Leben im Jenseits darf nicht dazu dienen, den Wert des irdischen Lebens klein zu reden, so wie wir das derzeit oft erschreckend in den Indoktrinatonsriten für Selbstmordattentäter erleben. Die Alte Kirche hatte durchaus Mühe, einem fanatischen Märtyrertum 
Einhalt zu gebieten. Gleichzeitig aber muss auch darauf geachtet werden, dass ein starker Akzent auf den Schutz des irdischen Lebens nicht zu einer Vergötterung des Diesseits und einer Relativierung des Jenseits führt. Schließlich gilt es auch zu bedenken, dass das irdische Leben selbst nicht unter der Hand einen göttlichen Charakter bekommt, dem gegenüber sich der einzelne Mensch in seiner Lebensführung unbedingt verpflichtet zu sehen hätte ${ }^{5}$.

\section{Theologie als Kunst des Abwägens}

Vor diesem Hintergrund besteht die Kunst der theologischen Reflexion für eine Urteilsbildung im Bereich der Sterbehilfe und der Sterbebegleitung darin, die Balance zwischen verschiedenen Fehldeutungen zu wahren. Von der Balance zwischen Diesseits und Jenseits, zwischen irdischem und ewigem Leben war dabei schon die Rede. Ebenso aber gilt es auch die Balance zu wahren zwischen dem Gedanken, dass wir unser Leben Gott verdanken und dem Postulat menschlicher Freiheit. „Zwar ist nach biblischem Verständnis Gott allein Herr über Leben und Tod. Aber das Handeln Gottes und dasjenige des Menschen sind kategorial ebenso voneinander $\mathrm{zu}$ unterscheiden wie aufeinander zu beziehen."6 Insbesondere lässt sich aus den Aussagen über das Handeln Gottes nicht unmittelbar auf das Handeln des Menschen schließen, ebenso wenig wie aus den Bestimmungen über die Selbstverortung im Glauben, aus Sätzen, die das Verhältnis des einzelnen Gläubigen gegenüber Gott zum Gegenstand haben, direkt Kriterien zur Klassifikation und Beurteilung von Handlungen abgeleitet werden können. Gott als den Herrn über Leben und Tod zu benennen kann daher noch nicht zu Aussagen darüber führen, wo die Grenzen zwischen dem Zulässigen und dem zu Unterlassenden liegen. Die Rede vom Tod als „Geschick“ führt dabei leicht in die Irre7. Denn ganz offenkundig kann mit dieser Rede ja nicht gemeint sein, die Praxis der Unfallrettung und der notärztlichen Versorgung in Frage zu stellen. Wer vom Tod als „Geschick“ spricht, der müsste auch eine plausible Antwort bieten können auf die Theodizee-Problematik. Oder warum sollte der Tod eines Kindes bei einem Autounfall nicht ebenso dem Willen Gottes entsprungen sein wie die Pflicht eines Schwerstkranken, gegen seinen verlorenen Lebensmut das Ende des eigenen Sterbeprozesses abzuwarten? Kirchliche Stellungnahmen sind hier häufig von einer

\footnotetext{
5 Interessanterweise spielte diese Unterscheidung schon bei der Bildung eines evangelischen Urteils zur Frage des Schwangerschaftsabbruchs eine Rolle; vgl. dazu exemplarisch Hans-Christian v. Hase (1962): Tage im Buch des Lebens. Das Recht des Ungeborenen - theologisch gesehen. In: Anstösse. Berichte aus der Arbeit der Evangelischen Akademie Hofgeismar, 104-113, 111. Hase konstatierte, die Frau sei „nicht Sklavin eines ,Allebens', sondern verantwortlicher Mensch“. 6 Verantwortung für das Leben. Eine evangelische Denkschrift zu Fragen der Biomedizin. Im Auftrag des Evangelischen Oberkirchenrats A. und H.B. der Evangelischen Kirche A. und H.B. in Österreich erarbeitet von Ulrich H. J. Körtner in Zusammenarbeit mit Michael Bünker, Wien 2001, Ziff. 3.1 .2

7 Vgl. dazu insbesondere auch die kritischen Anfragen von Klaus-Michael Kodalle (wie Anm. 4), $224 \mathrm{f}$.
} 
problematischen Inkonzinnität gekennzeichnet, die letztlich einem mythischen Weltbild entspricht, in dem auf der Ebene individuellen Handelns alles durch Gott determiniert erscheint. Ein solches Denken steht aber keineswegs im Einklang mit dem Gedanken der Welt als Schöpfung, sondern in unübersehbarem Kontrast dazu. Die Welt als Schöpfung zu begreifen bedeutet, zwischen Schöpfer und Geschöpf, aber auch zwischen Gott und Natur zu unterscheiden. Aus der Geschöpflichkeit des Menschen ist die Verantwortung des Menschen abzuleiten, nicht aber der Imperativ, sich vollends in sein Schicksal zu ergeben. In dieser Fähigkeit zu Freiheit und zur Verantwortung ist zugleich die dem Menschen eigene Würde begründet. Insofern ist es eine unzulässige Verkürzung der Problematik, wenn das Orientierungskriterium der Menschenwürde nur mit einer Ablehnung der Sterbehilfe in Verbindung gebracht wird.

Damit ist der dritte Bereich angesprochen, in dem die theologische Reflexion und damit auch die theologische Ethik nach einem wohl balancierten Ausgleich suchen müssen: zwischen Fürsorglichkeit und Freiheit. Dabei ist, der vorherrschenden Tradition der evangelischen Ethik entsprechend, in den Äußerungen der evangelischen Kirchen die Perspektive der Fürsorglichkeit prädominant. Wenn ich recht sehe, dann ist die Position der evangelischen Kirche im Blick auf die Sterbehilfe und auch die Reichweite von Patientenverfügungen ganz wesentlich durch das Bemühen gekennzeichnet, dem schwachen und verlöschenden Leben Schutz zu verleihen. Diese Perspektive der Fürsorglichkeit verbindet auch die durchaus unterschiedlichen, ja widersprüchlichen Positionen im Protestantismus in Bezug auf den Schutz des Lebens: War es in den 1970er Jahren das Engagement für die Frauen, in denen man die schwächsten Glieder in der Diskussion um den Schwangerschaftsabbruch sah, so wandelte sich dies unter dem Eindruck einer drohenden technischen Verfügungsgewalt über den Embryo, die sodann auch im Protestantismus zu einem strikten Embryonenschutz und einer teilweisen Revision der Einstellung gegenüber dem Schwangerschaftsabbruch führte. Derzeit fokussiert sich die Debatte auf die schwer Kranken und Sterbenden, denen gegenüber die Kirche als Fürsprecherin agieren möchte. Die strikte Ablehnung der Sterbehilfe soll frühzeitig ein Bollwerk aufbauen gegenüber möglichen gesellschaftlichen Tendenzen, auf schwer Kranke Druck auszuüben, doch möglichst früh aus dem Leben zu scheiden und so niemandem zur Last zu fallen; mehr noch: Sie soll auch die Einzelnen vor sich selbst und der verkehrten, weil sich gegen das eigene Leben wendenden Selbstbestimmung schützen. Dabei verfälltt man jedoch leicht in eine Haltung, die nun die Fürsorglichkeit gegen die Selbstbestimmung des Einzelnen ausspielen möchte ${ }^{8}$.

Glanz und Elend des Menschen liegen in seiner Fähigkeit, das eigene Leben gestalten zu können und gestalten zu müssen. Die besondere Brisanz der Diskussion um die Sterbehilfe in der Gegenwart resultiert nun eben daraus, dass das

8 Vgl. Sterben hat seine Zeit, $14 \mathrm{ff}$. 
unter den Bedingungen der Moderne Sterben selbst zu einem zu gestaltenden Prozess geworden ist. Die Fortschritte der Medizin haben es ebenso wie die Individualisierung und Pluralisierung von Lebensformen und Lebensentwürfen mit sich gebracht, dass die Behandlungsmöglichkeiten und damit auch die Entscheidungsnotwendigkeiten in der letzten Lebensphase signifikant zugenommen haben. Darum stellen sich die Fragen von Endlichkeit, Sterben und Tod in modernen Gesellschaften als praktische Fragen, die auf Gestaltungsmöglichkeiten und Handlungsoptionen abheben - und genau hier entzünden sich die Konflikte. Diese Konflikte liegen dabei auf zwei verschiedenen Ebenen: Sie betreffen die Art der Gestaltung des Sterbeprozesses und zugleich auch die Frage, wer das Deutungsmonopol für diese Gestaltung innehat. Waren es in der frühen Moderne die klassischen Professionen (Ärzte, Priester, Juristen), die nicht nur ein Deutungsmonopol über den Sterbeprozess innehatten, sondern auch ein spezifisches Ethos bei ihrer Klientel hervorbringen konnten, werden die Sterbenden nun selbst zu Akteuren. Die daraus resultierende Selbstbestimmung wird im Bereich von Kirche und Theologie zutiefst als ambivalent wahrgenommen, ebenso wie der Protestantismus seit jeher zur Freiheit des Einzelnen ein gespaltenes Verhältnis einnahm. Im Engagement der Kirchen für die Patientenverfügung wird diese Ambivalenz deutlich sichtbar, insofern hier zum einen die Berechtigung und Notwendigkeit individueller Gestaltung akzentuiert wird, zum anderen aber zugleich durch die Ausarbeitung und, wichtiger noch, die breite Verteilung einer Musterverfügung die individuelle Gestaltungsmöglichkeit rückgebunden werden soll an eine kirchlich ausgearbeitete Vorlage.

Angesichts der vielfältigen Szenarien am Lebensende und der eben skizzierten Notwendigkeit, das eigene Sterben als eine individuelle Gestaltungsaufgabe zu begreifen, kann es nicht verwundern, dass auch in der christlichen Patientenverfügung letztlich der Respekt vor der Individualität des Sterbevorgangs die entscheidende Rolle einnimmt. Gleichsam als ein integrierendes Paradigma formuliert die von den beiden großen Kirchen herausgegebene Vorlage: „Ich möchte in Würde und Frieden sterben können“. Die Orientierung an der Würde soll dabei offenbar als Maßstab auch für diejenigen Entscheidungssituationen dienen, die in den einzelnen vorgeschlagenen Handlungsanweisungen nicht geregelt werden konnten. Jedoch: In zahlreichen erläuternden Publikationen, Verlautbarungen zur Thematik, besonders in der bereits zitierten Stellungnahme der Kammer für öffentliche Verantwortung der EKD wird gegenüber der grundsätzlichen Ausrichtung an den individuellen Vorstellungen des Einzelnen versucht, eine normierende Interpretation für die Formel ,in Würde sterben“ zu gewinnen. Demgegenüber möchte ich das Individualisierungsparadigma stärker akzentuieren und plausibel machen, dass es auf die Frage, was es bedeutet, in Würde zu sterben, nur eine Antwort gibt: keine - und das heißt natürlich: keine allgemeingültige. Was würdiges Sterben bedeutet, ist so vielfältig wie die korrespondierenden Lebensentwürfe. Aber genau darin liegt nun offenbar doch eine Antwort auf die von mir gestellte Frage: Wür- 
diges Sterben bemisst sich offenbar an den Vorstellungen über ein würdiges Leben. Von dieser These aus möchte ich versuchen, mich der Frage nach dem würdigen Sterben und damit auch der Frage nach der Sterbehilfe genauer anzunähern.

\section{Orientierung an der Menschenwürde als Brücke zwischen Leben und Sterben}

Wer auf die Würde als maßgeblichen Orientierungspunkt auch beim Sterben rekurriert, schlägt damit offenbar eine Brücke zwischen Leben und Sterben. Im Sterben soll, so lautet die implizite Weisung, leitend sein, was auch für das Leben als oberste Orientierungsnorm gilt, nämlich die Ausrichtung an der Würde des Menschen. Unbeschadet dessen, dass hierbei noch vollkommen offen ist, welche Verhaltensformen sich mit der Würde vereinbaren lassen, lässt sich daher schon eine erste Präzisierung vornehmen. Würdiges Sterben bedeutet, die Kontinuität zu wahren zwischen dem Leben und dem Sterben. Auch angesichts der äußersten, nicht mehr gestaltbaren Grenze unserer Existenz sollen dieselben Maßstäbe gelten, wie sie auch zuvor für die eigene Lebensführung charakteristisch waren. Über den Begriff der Würde wird somit die Brücke geschlagen zwischen den etablierten Verhaltensformen, Normen und Werten, die die Identität des Einzelnen im Leben beschrieben haben, und den besonderen Herausforderungen, die durch die Situation der Todesnähe neu gestellt werden. Die Orientierung an der Menschenwürde soll somit die Stetigkeit im Handeln und Erleiden auch in der Situation des Sterbens sicherstellen. Diese Stetigkeit ist ja auch sonst ein wichtiges Charakteristikum unserer ethischen Entscheidungsfindung: Vor die Frage gestellt, wie wir uns in einer bestimmten, neuen Situation verhalten sollten, fragen wir danach, wie wir uns bislang in einer ähnlichen Konstellationen verhalten haben. Damit versuchen wir, unser Handeln möglichst evolutionär weiterzuentwickeln: Neues muss sich als integrierbar in bisherige Entscheidungen erweisen können und gleichzeitig darf die eigene Vergangenheit sich nicht als Hemmschuh für die Weiterentwicklung des eigenen Lebensentwurfs gerieren. In dieser Perspektive lässt sich „würdiges Sterben“ dekodieren als die Aufforderung, Sterben in Kontinuität und Stetigkeit zum eigenen Leben zu gestalten.

Zugleich mit dieser Beschreibung werden jedoch weitere inhaltliche Konturen sichtbar, was mit „würdigem Sterben“ gemeint ist. Denn im Sterben nach den Maßregeln zu handeln, die auch für die eigene Lebensführung bestimmend waren, ist gleichbedeutend mit dem Respekt vor dieser höchstindividuellen Situation. Die Binsenweisheit, dass jeder seinen Tod sterben muss, gewinnt darin andere, deutlichere Konturen. Sie bedeutet dann nämlich, dass das Sterben nicht nach deindividualisierenden, gleichförmigen Prozessregeln erfolgen soll. Die Aussage, dass jeder seinen eigenen Tod sterben muss, ist darum nicht nur als eine deskriptive Aussage zu verstehen, sondern als eine normative. Wenn Luther davon sprach, 
dass der Glaube immer nur ein individueller sein könne, weil wir im Sterben auch unvertretbar seien, weil eben jeder seinen eigenen Tod sterben müsse ${ }^{9}$, so gilt das auch für die ethischen Problemlagen am Ende des Lebens. Würdiges Sterben heißt, Raum für eine individuelle Gestaltung des Sterbens zu lassen. Wenn ich recht sehe, ist dieser Grundsatz von der Rechtsprechung nachdrücklicher rezipiert worden als von der Ethik, gerade auch der kirchlich formulierten Ethik. Denn während hier häufig die grundsätzliche, über einzelnen Interessen liegende Schutzfunktion des Rechts eingeklagt wird, erweisen sich die Juristen als überzeugte Verfechter individueller Autonomie auch in Grenzsituationen. Sie betonen im Anschluss an das Konzept der Menschenwürde die Individualität medizinethischer Entscheidungen und wollen das Recht lediglich als die magna charta des Einzelnen, als Schutz gegen überbordende Interessen der Allgemeinheit verstanden wissen ${ }^{10}$.

Sterben ist eine höchstindividuelle Angelegenheit. Durch die moderne Medizin wird - und daraus resultiert wohl auch die Ambivalenz, die ihr entgegengebracht wird - auf der einen Seite die Phase des Sterbens mit den entsprechenden Notwendigkeiten individueller Entscheidungen erst erzeugt; auf der anderen Seite stellt jedoch die Medizin diese Individualisierung selbst auch immer wieder in Frage. Denn die moderne Medizin operiert ja mit der Hypothek einer grundsätzlichen Paradoxie: Sie konnte die unbestreitbaren Fortschritte nur dadurch erreichen, dass sie vom Einzelnen in seiner Individualität abstrahierte und die Vorgänge seines Körpers nach dem Paradigma eines maschinengleichen Regelkreises verstehen lernte. Angesichts der Möglichkeiten moderner Intensivmedizin wächst jedoch die Befürchtung, als Patient zum Objekt technischer Möglichkeiten und

9 So etwa in Predigt am Sonntag Invocavit am 9. März 1522. In: Ders. (21964): Luther deutsch. Hrsg. von Kurt Aland. Bd. 4: Der Kampf um die reine Lehre. Göttingen, S. 61

10 Diese Sicht prägt die Rechtsprechung in dieser Angelegenheit schon seit längerem: So hält das OLG München 1987 fest: „Das Selbstbestimmungsrecht des Patienten schließt auch die Selbstbestimmung zum Tode ein. Hinsichtlich lebensverlängernder Maßnahmen bindet der vom urteilsfähigen Patienten ausgesprochene Verzicht den Arzt auch dann, wenn der Patient im voraussehbaren Verlauf der Krankheit das Bewusstsein verliert und keine wesentliche Veränderung der seiner Erklärung zugrunde liegenden tatsächlichen Umstände erkennbar ist, weil die Entscheidung gerade auch für dieses Stadium getroffen wurde, wie auch umgekehrt die Einwilligung zum Heileingriff nicht ihre rechtfertigende Wirkung mit Eintritt der Bewusstlosigkeit verliert"; OLG München, JA 1987, 579, 583. 1991 konstatiert der 3. Strafsenat des BGH: „Kann der todkranke Patient nicht mehr selbst entscheiden und wird für ihn auch kein Pfleger bestellt, [...] so ist sein mutmaßlicher Wille und nicht das Ermessen der behandelnden Ärzte rechtlicher Maßstab dafür, welche lebensverlängernden Eingriffe zulässig sind und wie lange sie fortgesetzt werden dürfen. Die Ausschöpfung intensivmedizinischer Technologie ist, wenn sie dem wirklichen oder anzunehmenden Patientenwillen widerspricht, rechtswidrig“; BGHSt 37, 376, 378. 1999 nimmt das LG München I den Gedanken vom Sterben als „höchstpersönlicher Angelegenheit“ auf; LG München I, 13 T 478/99. Zur Problematik vgl. insbes. Frank Saliger (2004): Sterbehilfe und Betreuungsrecht. Medizinrecht, 22, 237-245 sowie die Zusammenstellung bei Klaus Ulsenheimer (2000): Aktive und passive Sterbehilfe aus der Sicht der Rechtsprechung. Internist, 41, 648-653. Kritisch zu dieser Tendenz: Axel W. Bauer (2003): Futility-Debatte in der Onkologie. Ein ethisches und rechtliches Problem. Onkologe, 9, 1325-1334. Zum Ganzen vgl. auch die instruktiven Ausführungen bei Volker Lipp (2000): Freiheit und Fürsorge: Der Mensch als Rechtsperson. Zur Funktion und Stellung der rechtlichen Betreuung im Privatrecht (=Jus Privatum 42). Tübingen, S. 164ff. 
ärztlicher Forscherinteressen zu werden. Darum wird im Interesse der eigenen Selbstbestimmung der Ruf nach den Grenzen für intensivmedizinische Maßnahmen laut. Die intensive Diskussion um Betreuungsverfügungen und Patiententestamente ist ein deutliches Indiz dafür. Würdiges Sterben steht hier ganz offenbar als Chiffre für die Forderung, selbst Herr des Prozesses zu bleiben. Das Sterben hat damit zugleich Anteil an den Individualisierungsprozessen, die für moderne Gesellschaften charakteristisch sind. Auch dieser Aspekt dürfte sich unschwer abbilden lassen auf das Paradigma, das Sterben nach der Maßgabe des eigenen Lebensentwurfs zu gestalten.

Vor diesem Hintergrund erscheint auch nachvollziehbar, dass die Auseinandersetzungen um Intensivmedizin und deren Grenzen nur einen Teilbereich der mit der Individualisierung des Sterbens verbundenen Konsequenzen abdecken. Gerade im Blick auf die Bestattungspraxis sind hier intensive Kontroversen geführt worden, spätestens seit den Debatten um das am 4. Juni 2003 verabschiedete neue Bestattungsgesetz für Nordrhein-Westfalen. Bereits 1998 hatte eine vom Institut für Demoskopie Allensbach durchgeführte Repräsentativumfrage „Tod und Grabkultur" als Ergebnis erbracht, dass ein Viertel aller Deutschen ihre Grabstätte ohne behördliche Einschränkungen selbst gestalten möchte, $20 \%$ möchten Gräber auf ihrem Privatgrundstück anlegen ${ }^{11}$. Ohne Zweifel spiegelt diese Ablehnung behördlicher Normierung der Bestattungspraxis auch die zunehmende Individualisierung des Sterbens, und zwar, noch einmal sei es betont, einer Individualisierung in deskriptiver und normativer Perspektive.

\section{Die bewusste Gestaltung des Sterbens als Teil des eigenen Lebensentwurfs}

Würdiges Sterben ist ein Sterben, das sich als integrierbar in die eigene Lebensführung erweist. Nur unter diesen Bedingungen erscheint es möglich, den Tod als Teil des Lebens - und zwar des eigenen Lebens - zu begreifen und schließlich, wenn man das überhaupt so ausdrücken kann, auch als persönlichen Tod annehmen zu können. „Sie starb, wie sie es sich immer gewünscht hatte“ - lautet die entsprechende Beschreibung eines als würdig empfundenen Sterbeprozesses. Dieses Begreifen des Sterbens als des individuellen Sterbens stellt eine große Herausforderung dar, weil wir es - glücklicherweise - gewohnt sind, das Sterben zwar als zum Leben, nicht aber als zum eigenen Leben gehörig zu verstehen. Möglicherweise ist es sogar so, dass das Wissen um die eigene Sterblichkeit und den eigenen Tod gar nicht in den eigenen Lebensvollzug integriert werden kann. „So konnte“, schrieb Sigmund Freud, ,in der psychoanalytischen Schule der Ausspruch gewagt werden: Im Grund glaube niemand an seinen eigenen Tod oder, was dasselbe ist:

11 Tod und Grabkultur - eine Repräsentativumfrage des Instituts für Demoskopie Allensbach, im Auftrag des „forums grabkultur“, durchgeführt im Mai 1998 an 2177 Befragten, 34 f. 
im Unbewussten sei jeder von uns von seiner Unsterblichkeit überzeugt.“12 Es gehört zu den Errungenschaften der Moderne, dass es gelungen ist, das Bewusstsein eigener Sterblichkeit weitgehend auf Distanz zu halten. Sicher, Sterben und Tod ist gerade in einer medialisierten Welt allgegenwärtig. Aber für jeden einzelnen gilt doch, was Leo Tolstoj im Tod des Iwan Iljitsch 1886 so anschaulich beschreibt: Jenes bekannte Beispiel für Syllogismen - alle Menschen sind sterblich. Caius ist ein Mensch. Also ist Caius sterblich - ,war ihm sein ganzes Leben hindurch rechtmäßigerweise lediglich als auf Caius anwendbar vorgekommen, keinesfalls aber auf ihn, Iwan Iljitsch, selber. Jenes war der Mensch überhaupt, und für diesen war das Gesetz völlig gerechtfertigt; er indes war nicht Caius und ebenso wenig der Mensch an sich. Caius, der war in der Tat sterblich, und wenn er starb, so war es ganz in der Ordnung; ich dagegen, ich, Wanja, ich Iwan Iljitsch, mit allen meinen Gedanken und Gefühlen, - bei mir ist es nun einmal eine ganz andere Sache." Über das Sterben nachzudenken ist offenkundig etwas anderes als über das eigene Sterben zu reflektieren - und diese Differenz mahnt noch einmal dazu, die Individualität des Sterbegeschehens und seiner Gestaltung ernst zu nehmen. Dabei gibt es freilich eine konstitutive Grenze der Reflexion. Wir können immer nur aus der Perspektive des Lebens auf das Sterben und auch den Tod blicken. Ludwig Wittgenstein hat darum mit der ihm eigenen Präzision festgehalten: „Der Tod ist kein Ereignis des Lebens. Den Tod erlebt man nicht. “13 Alles Nachdenken über den Tod und das Sterben kann daher nur die Gestaltung des Lebens zum Gegenstand haben. Und, in Aufnahme und Weiterführung des zuvor Gesagten: Das Nachdenken über das eigene Sterben und den eigenen Tod kann nur dazu anleiten, das eigene Sterben als Teil des eigenen Lebens zu gestalten. Insofern scheint es mir auch wenig hilfreich zu sein, im Blick auf die kontroverse Unterscheidung und Bewertung von aktiver und passiver Sterbehilfe die Grenzlinie dort zu ziehen, wo der Tod im einen Fall als Tat, im anderen Fall als Ereignis wahrgenommen wird: Während das „Warten auf den Tod“ als ethisch unproblematisch klassifiziert wird, steht dessen bewusstes Herbeiführen in der Kritik. Möchte man die spezifische Spitze des Menschenwürdearguments ernst nehmen, dann muss die Gestaltung des Sterbens bis hin zum Herbeiführen des eigenen Todes gerade als Respekt vor der Würde des Einzelnen auch im Sterben gedeutet werden. Denn seit seiner detaillierteren Ausarbeitung in der Renaissancephilosophie markiert das Menschenwürdeparadigma ein Verständnis des Menschen, der sich nicht nur als das Produkt seiner natürlichen Gegebenheiten begreift, sondern seinen Lebensentwurf dem eigenen Willen gemäß zu gestalten weiß. Wenn heute häufig mit dem Verweis auf den Respekt vor der Menschenwürde oder die Unantastbarkeit der Menschenwürde das Würdeargument als Widerlager gegen zu weit reichende Handlungsspiel-

12 Sigmund Freud: Zeitgemäßes über Krieg und Tod. In: Ders. (1974): Studienausgabe (Conditio humana. Ergebnisse aus den Wissenschaften vom Menschen). Bd. IX. Frankfurt/M., S. 49 13 Ludwig Wittgenstein: Tractatus-logico-philosophicus 6.4311, zit. nach ders. (1984): Werke in acht Bänden. Frankfurt /M., Bd. 1, S. 84 
räume des Menschen in Anschlag gebracht wird, bedeutet dies gerade keine Befestigung der Würdevorstellung, sondern die Unterminierung ihrer eigenen Grundlagen. Wohl verbietet das Würdeargument staatliche Eingriffe in die Sphäre der einzelnen Persönlichkeit - die Rechtsordnung darf dem Einzelnen nicht eine bestimmte Gestaltung des Lebens aufzwingen, von der Berufswahl bis zum Austragen einer Schwangerschaft. Diese Einschränkung kann aber nicht auf das Verhältnis des Einzelnen zu sich selbst und zu seinem Lebensentwurf übertragen werden. Hier bedeutet der Hinweis auf die Menschenwürde, das eigene Leben eben nicht nur als Schicksal, sondern als Gestaltungsaufgabe zu begreifen. Zu dieser Möglichkeit und Notwendigkeit, das eigene Leben zu gestalten, gehört es aber auch, das eigene Sterben angesichts des Bewusstseins individueller Endlichkeit zu gestalten. Erst aus dem mit der Würdevorstellung verbundenen Postulat der Freiheit ergibt sich überhaupt die Möglichkeit verantwortbaren, sittlich zu qualifizierenden Handelns - gemeinsam übrigens mit der Möglichkeit auch des fehlerhaften und schuldhaften Handelns. Freiheit und Verantwortung, Würde und Schuld sind jeweils komplementäre, aufeinander bezogene Begriffe. Das bedeutet freilich, dass die Orientierung an der Würde des Menschen nicht automatisch schon das gute Handeln nach sich zieht. In der theologischen Tradition ist dies in der Rede von Freiheit und Sünde zum Ausdruck gebracht worden, die beide mit der Geschöpflichkeit des Menschen gegeben sind. Insofern kann es - scheinbar paradox - durchaus sein, dass die Orientierung an der Würde des Einzelnen ein Schuldhaftwerden nach sich zieht. Doch diese Paradoxie wäre nur um den Preis einer anderen, weiterreichenden Paradoxie aufzulösen, der Paradoxie nämlich, im Namen eines guten Lebens auf die Freiheit des Einzelnen in Respekt vor seiner Würde zu verzichten.

\section{Das eigene Sterben unter den Bedingungen moderner Medizin}

Würdiges Sterben, so hatte ich gesagt, bedeutet, das Sterben der eigenen Gestaltung zu unterwerfen und es nicht einfach als ein zu Erduldendes, als fremdbestimmende Macht zu begreifen. Dabei führt jedoch, wie bereits angesprochen, die Wahrnehmung dieser Gestaltungsaufgabe unter den Bedingungen der Moderne unweigerlich in eine Paradoxie: Die Möglichkeiten, das eigene Leben als Aufgabe und nicht nur als Schicksal zu begreifen, sind in hohem Maße abhängig von einer Sichtweise, die den Menschen eben nicht als ein Subjekt und damit als Träger von Würde wahrnimmt, sondern ihn gerade als Objekt auffasst. Das gilt für die vielfachen Verobjektivierungsprozesse des täglichen, arbeitsteiligen Lebens, in denen wir einander stets nur als Mittel dienen, es gilt aber in besonderem Maße für die Medizin selbst, in der Fortschritte gerade durch die Verobjektivierung der Einzelnen zu Körpern, nicht zuletzt zu Studienobjekten, erreicht werden. In diesem 
Sinne ist übrigens auch darauf hinzuweisen, dass die beiden Paradigmen der Patientenautonomie und des ärztlichen Paternalismus keineswegs unvereinbare Gegensätze darstellen. Vielmehr kann es gerade Ausdruck von Selbstbestimmung und Autonomie sein, sich der paternalistisch-verobjektivierenden Struktur der Medizin zu unterstellen - um der Wahrung oder Wiederherstellung eigener Autonomie willen. Diese Struktur wird so lange akzeptiert, solange sie vereinbar ist mit der Intention des Patienten, das medizinische System in der Absicht in Anspruch zu nehmen, seine aus der Würde abgeleiteten Freiheits- und Handlungsspielräume zu erweitern. Allerdings kann das medizinische System dieser Intention gerade am Ende des Lebens nicht mehr gerecht werden. Professionalisierung im Sinne einer Erweiterung von Handlungsspielräumen schlägt dann um in eine Entmündigung des Patienten: Was seiner Freiheit und Würde dienen sollte, droht beide zu negieren.

Diese Paradoxie nötigt dazu, die Erwartungen und Rollenzuschreibungen im Blick auf Sterbende und Kranke präziser zu bedenken. Denn solange Sterbende als Kranke aufgefasst werden, scheint eine Deautonomisierung im Interesse der Wiederherstellung ihrer Gesundheit legitim. Wo die Bevormundung der Sterbenden durch das System der Intensivmedizin jedoch kritisiert wird, geschieht das gerade aus dem Bewusstsein heraus, dass eine solche Negierung des Respekts vor der Gestaltung des eigenen Lebens nicht mehr hinzunehmen ist. Sterbende sind jedoch keine Kranken; zwar stirbt ein Teil der Menschen als Kranke, dennoch ist das Sterben Bestandteil menschlicher Existenz und nicht eine pathologische, therapierbare Devianz. Dementsprechend gelten - in Analogie zu chronisch Kranken - andere Regeln und mit ihnen muss anders umgegangen werden. Bei Patienten, die schon eine längere Krankheitsgeschichte hinter sich haben, kommt es zu ReNormalisierungseffekten, die die handlungsleitende Bedeutung der Krankheit zurücktreten oder auch gar nicht erst zum Vorschein kommen lassen. Dieses Phänomen ist mit den Kategorien von „Tabuisierung“ und „Verdrängung“ nur unzureichend beschrieben. Vielmehr gehört es zu den notwendigen Akkomodationsleistungen eines unheilbar Kranken, die alltagsstörende Dimension einer Krankheit zurücktreten zu lassen.

Folgt man dem Gesundheitsbegriff der phänomenologischen Soziologie, demzufolge man Gesundheit immer dann hat, wenn ihr Fehlen nicht auffällt und andere Alltagspraktiken nicht stört ${ }^{14}$, ist es für einen chronisch oder final Kranken notwendig, die Krankheit zum Bestandteil des eigenen Alltags werden zu lassen und gerade darin das Sterben als einen Teil des eigenen Lebens aufzufassen. Im Sinne meiner Ausgangsthese wäre mithin auch hier ein würdiges Sterben nach den leitenden Vorstellungen des eigenen Lebens zu bemessen, von der Integration in die Lebensvorstellungen des Einzelnen.

14 Vgl. Uta Gerbardt: Lebensweisen und Gesundheitsorientierungen: Methodologische Probleme. In: Reinhard Gawatz \& Peter Novak (Hrsg.) (1993): Wissenschaftliche und alltagspraktische Gesundheitskonzepte. Ulm, S. 73-96, S. 84 
Diese Alltagsintegration ist selbst jedoch ein vielschichtiger Prozess, und dabei kann in der letzten Lebensphase der Alltag durchaus die Wirklichkeit der Klinik sein. Deutlich sichtbar ist dies etwa an der mehrschichtigen Semantik, die das Postulat „Ich möchte zu Hause sterben“ bei final Kranken erfährt: Die große Diskrepanz zwischen diesem häufig geäußerten Wunsch und den tatsächlichen Sterbeorten kann nicht adäquat mit dem Verweis auf die mangelnde Sensibilität des Medizinsystems erklärt werden; vielmehr wird offenbar das Krankenhaus selbst im Verlauf der Krankheit zum Ort des Alltags und damit zum Zuhause. Aus der Perspektive der Patienten scheint es darum offenbar konsequent zu sein, nun auch das Sterben selbst als Aufgabe des Medizinsystems zu begreifen. Die konstant hohen Zustimmungsraten zur (aktiven) Sterbehilfe dürften hierin ihre Ursache haben. Eigene Untersuchungen an 272 Tumorpatienten in Thüringen haben ergeben, dass in der Perspektive der Betroffenen der Wunsch nach Sterbehilfe, und zwar sowohl nach aktiver wie auch nach passiver und indirekter Sterbehilfe, korreliert mit dem Bestreben, sich als Sterbender wie ein Patient zu verhalten und sich damit in das medizinische System zu integrieren. Der Wunsch nach Sterbehilfe lässt sich demnach nicht als Wunsch nach dem Ausstieg aus der medizinischen Versorgung interpretieren, sondern vielmehr als Folge einer konsequenten Einordnung in das System der Medizin, als Adaptionsleistung an die nunmehr für den einzelnen terminal Kranken geltenden Rahmenbedingungen. Dieses Sterben kann in der Fluchtlinie meiner hier vorgestellten Argumentation als ein würdiges Sterben begriffen werden.

Allerdings - das sollte nach dem Vorangegangenen deutlich sein - kann aus einer anderen Perspektive das Sterben in der Klinik auch wahrgenommen werden als der Inbegriff eines unwürdigen Sterbens. Das ist in der Regel dann der Fall, wenn durch das Fehlen eines geregelten Statusübergangs zwischen der Krankenund Sterbendenrolle es die systemischen Bedingungen in der klinischen Sterbesituation unmöglich machen, dass das Sterben als Teil des Lebens aufgefasst werden kann. Dieses Defizit ist es, das häufig irreführend als Phänomen der Todesverdrängung in der Gesellschaft beschrieben wird. Tatsächlich ist es zusammen mit der Entwicklung der modernen Medizin zu einer Medikalisierung des Sterbens gekommen, die dazu führt, dass immer mehr Menschen als Kranke, das heißt als Patienten sterben. Sie müssen dabei - häufig gemeinsam mit ihren Angehörigen erleben und auch erleiden, dass das Medizinsystem nur wenige Rezeptoren für die Bedürfnisse des Sterbens bereitstellen kann, bis dahin, dass es nur selten gelingt, die entsprechenden Statusübergänge adäquat, d.h. eingebettet in einen deutenden und entlastenden Ritualzusammenhang, zu gestalten. 


\section{Nicht normieren, sondern deuten: Die Paradoxie der Gestaltung des eigenen Sterbens und der christliche Glaube}

Wenn würdiges Sterben im Kern bedeutet, das Sterben als Teil des eigenen, individuellen Lebens zu begreifen und zu gestalten, dann muss jedoch abschließend noch auf eine unausweichliche Frage eingegangen werden: Wie kann etwas als Teil von Individualität und Persönlichkeit aufgefasst werden, das die Zerstörung und Negation dieser Person zur Folge hat? An dieser Stelle scheint mir die bleibende Bedeutung religiöser Deutungsangebote des Todes zu stehen. Religiöse Begleitung des Sterbens muss zum Ausdruck bringen, dass die Integrität der Person nicht mit ihrem Tod endet. Dies besagt in ihrem Kern die christliche Rede von der Auferstehung, und in diesem Zeichen steht auch der christliche Trauerritus. Dabei ist es von zentraler Bedeutung, dass diese Deuteangebote nicht andemonstriert und schon gar nicht zum allgemeinen Leitsatz erhoben werden können. Sie können lediglich helfen, für sich selbst eine Perspektive zu gewinnen, die es möglich macht, die zerstörende Kraft des Todes in eine individuelle Auffassung vom Leben zu integrieren. So gilt auch hier: Ob das Sterben ein würdiges Sterben ist, bemisst sich letztlich an der höchstindividuellen Frage, ob der Einzelne für sich zu der Einschätzung gelangen kann, dass das Angebot einer Integration des Sterbens in das Leben, das etwa die religiöse Kultur bereit hält, für ihn eine Perspektive bereithält, die die Macht des Todes mitsamt der ihm entgegentretenden Bedrohung seiner eigenen Personalität und Würde bricht und übersteigt. Mit der Plausibilität einer solchen Deutungsvorstellung steht und fällt die Einschätzung des Sterbens als ein würdiges Sterben, mit ihr steht und fällt aber auch die Bedeutung der Religion. Vielleicht hatte Paulus dies vor Augen, wenn er an die Gemeinde in Korinth schrieb: Wenn aber Christus nicht von den Toten auferstanden ist, dann ist alles nichtig (1 Kor 15, 14). 


\section{Sterben wo und wie? Palliativmedizin - die vierte Dimension in der modernen Gesundheitsversorgung}

\section{Dietrich Kettler}

\section{Einleitung}

Die gegenwärtige Gesundheitsversorgung der Bevölkerung geschieht in drei unterschiedlichen dem Gesundheitszustand zugeordneten Abschnitten: der Prävention zur allgemeinen Verhütung oder zumindest Verzögerung des Auftretens von Krankheiten, der Kuration, Heilung bereits aufgetretener Erkrankungen und der Rehabilitation, das an die Akutversorgung anschließende Bemühen, die noch verbleibenden krankheitsbedingten Zustände zu beseitigen oder zumindest zu mildern. Nicht selten ist dieses ein lang dauernder Prozess mit je nach der Primärerkrankung erfolgreichem Ergebnis, der im Extremfall aber auch in völliger Pflegebedürftigkeit enden kann.

Nicht vorgehalten werden hingegen Strukturen und deren Finanzierung für die mit zunehmender Alterung der Bevölkerung stetig steigende Zahl von Patienten, die aufgrund ihres Krankheitsverlaufs nicht mehr definitiv geheilt werden können und unpassend oft auch als ,austherapiert" bezeichnet werden. Derartige Erkrankungszustände, z. B. ein progredientes Tumorleiden, das auf therapeutische Maß- 
nahmen nicht mehr anspricht, aber auch viele extrem belastende Endphasen anderer Erkrankungen (z. B. die chronische obstruktive Lungenerkrankung, die Terminalphase der Herzinsuffizienz und verschiedene neurologische degenerative Erkrankungen) finden in dem Gesundheitssystem unserer Gesellschaft keinen angemessenen Platz. Im Vorwort zur 2. Auflage ihres Lehrbuchs Palliativmedizin schreiben Husebø und Klaschik: „Zwei Drittel aller Sterbenden sind älter als 75 Jahre. Von ihnen sterben $40 \%$ mit einer Tumorerkrankung. Die Mehrzahl aller alten Patienten hat ein chronisches Leiden. In ihrer letzten Lebensphase leben und sterben Menschen häufig unter Bedingungen, die menschenunwürdig sind. Sie erhalten eine inkompetente medizinische und pflegerische Versorgung, weit weg von Hospizen, Palliativstationen oder ,Palliative Care (qualifizierte ambulante Versorgung). "

Es ist nur folgerichtig, dass viele Menschen die aktive Sterbebegleitung einem Lebensende unter Schmerzen und Würdelosigkeit einschließlich des Verlustes, ihre Selbstbestimmung realisieren zu können, vorziehen. In unseren Nachbarländern Belgien und den Niederlanden wurde diese Möglichkeit, die aktive Sterbehilfe (Tötung auf Verlangen), unter bestimmten Voraussetzungen legalisiert ${ }^{2}$. Aber auch in Deutschland waren nach einer FORSA-Umfrage $81 \%$ der Bevölkerung und einer Umfrage der Zeitschrift Stern mehr als $60 \%$ für die Möglichkeit einer aktiven Beendigung des eigenen Lebens. Die Diskussion über dieses Thema ist in vollem Gange, besonders was den assistierten Suizid und die Reichweite von Patientenverfügungen angeht. Die Palliativmedizin stellt sich als vierte Säule - neben Prävention, Kuration und Rehabilitation - unseres Gesundheitssystems der Herausforderung, unerträgliches Leiden unter jedwedem Verzicht auf kurative Maßnahmen zu mildern, Patienten in der letzten bedeutenden Phase des Lebens, dem Sterben, zu begleiten und auf diese Weise das Verlangen nach aktiver Beendigung des Lebens weitgehend zu vermeiden.

Dem verständlichen Wunsch der Mehrheit der Bevölkerung nach einer symptomarmen Sterbephase, die oft bis zu mehrere Monate und gelegentlich ein bis zwei Jahre dauert, und insbesondere nach einem Sterben unter würdigen Bedingungen steht das gegenwärtige Gesundheitssystem in unserem Land überwiegend hilflos gegenüber. Die Situation in großen amerikanischen Krankenhäusern wurde beispielhaft in der weltweit beachteten „Study to Understand Prognoses and Preferences for Outcomes and Risks of Treatment (SUPPORT) "3 untersucht. Die Support-Studie ergab folgende Kernergebnisse:

- Zwischen Ärzten und Patienten bzw. deren Familien fand keine ausreichende Kommunikation über das bevorstehende Sterben und medizinische Entscheidungen statt.

\footnotetext{
Husebo S \& Klaschik E (2000): Palliativmedizin. Grundlagen und Praxis. Berlin

2 S. Beitrag von A. Simon in diesem Band.

3 The Support Principal Inverstigator (1995): The study to Understand Prognoses and Preferences for Outcomes and Risks of Treatments (Support). JAMA, 274, 1591-1598
} 
- $38 \%$ der Patienten wurden auf Intensivstationen behandelt, z. T. mit maschineller Beatmung.

- $50 \%$ der Patienten hatten während der Sterbephase mittelstarke oder starke Schmerzen.

Eine darauf folgende 2. Phase der Intervention zur Verbesserung der Situation durch Einsatz von Support-Teams, die den Ärzten, dem Pflegepersonal, den Patienten und ihren Familien beratend zur Seite standen, erbrachte leider keine Verbesserung der beklagenswerten Situation. Das Fazit der Autoren war:

- Bei schwerkranken und sterbenden Patienten ist die Schmerzlinderung im Krankenhaus häufig insuffizient.

- Oft liegt vermeidbares Leiden vor.

- Häufig wird vergebliche Intensivtherapie durchgeführt.

- Die Interventionsstudie zur Verbesserung der Lebensqualität Sterbender verfehlte unter den gegebenen Bedingungen ihr Ziel.

Diese Studie erregte die amerikanische Öffentlichkeit in ungewöhnlichem Ausmaß, was sich in folgenden Schlagzeilen der führenden Presseorgane ausdrückte:

- Wünsche sterbender Patienten werden ignoriert, schwerkranke Patienten leiden unter der unpersönlichen Atmosphäre und dem starren Krankenhaussystem (Washington Post).

- Ärzte kennen nicht die Wünsche und Bedürfnisse sterbender Menschen (New York Times).

- Terminal care - too painful, too prolonged (Newsweek).

- Gnade eines guten Todes bleibt vielen Patienten verwehrt (Washington Post Health).

- Ungelöste Probleme, die Behandlung am Lebensende zu verbessern (JAMA).

Die Frage mag erlaubt sein, ob diese erschütternde Beschreibung der Krankenhausszene nur typisch für die US-amerikanische Situation ist oder nicht vielmehr den deutschen Verhältnissen entspricht.

Ohne dass entsprechende Daten für Deutschland vorliegen, kann nach vielerlei Erfahrungen von Patienten auf der einen und Behandlern auf der anderen Seite festgestellt werden, dass die Unterschiede zu den USA nicht so groß sind, wie man hoffen möchte.

Und wenn diese Zustände auch in unserem Gesundheitswesen im ambulanten wie im stationären Sektor Realität sind, ist dann nicht der Ruf nach Sanktionierung der aktiven Beendigung unerträglichen Leidens gerechtfertigt?

Die Alternative allerdings wäre, für viele Patienten unerträgliche Zustände am Lebensende durch eine Veränderung der Situation hin zu einem humanen Gesundheitssystem zu ersetzen, das den Menschen von der Geburt bis zum Tode in einem ganzheitlichen Ansatz und unter den verschiedenen Bedingungen seines Lebens und Leidens akzeptiert und dafür entsprechend adaptierte Versorgungskonzepte vorhält. 
Die moderne Medizin einschließlich des Medizinstudiums ist ganz überwiegend auf die Kuration ausgerichtet. Das Versagen der Kuration wird oft als Niederlage der Behandelnden empfunden. Der betriebsmäßige Charakter des Krankenhauses verstärkt die Tendenz zur kurativen Medizin und lenkt dadurch die Aufmerksamkeit von kranken Menschen selbst weg. Dies gilt natürlich besonders für den sterbenden Patienten, der in unserem Gesundheitsbetrieb keinen rechten Platz mehr findet. Seine Ansprüche liegen in einem Bereich, der durch die kurative Medizin nicht ausreichend abgedeckt wird.

An die Stelle der nicht mehr möglichen Heilung muss ein anderes Paradigma treten, das in der Palliativmedizin zusammengefasst ist. Die Palliativmedizin, die ihren Ausgang von der Hospiz- und Palliativbewegung in England nahm, wo Dame Cicely Saunders 1967 das St Christopher's Hospice gründete, wird von der WHO (1990) wie folgt definiert:

„Palliativmedizin ist die aktive, ganz̧heitliche Behandlung von Patienten mit einer progredienten, weit fortgeschrittenen Erkrankung und einer begrenzten Lebenserwartung zu der Zeit, in der die Erkrankung nicht mehr auf eine kurative Behandlung anspricht.

Die Beherrschung von Schmerzen, anderen Krankheitsbeschwerden, psycho-sozialen und spirituellen Problemen besitat höchste Priorität.

Die Behandlung strebt die bestmögliche Lebensqualität an, das Sterben soll weder beschleunigt noch binausgezögert werden. "

Palliativmedizin verfolgt Ziele, die sich auf einer herkömmlichen Krankenstation oder in einer Arztpraxis nicht oder nur ungenügend realisieren lassen. Sie

- respektiert die Würde des Menschen im Leben, im Sterben und danach,

- sorgt für Linderung von Schmerzen und weiteren belastenden Symptomen,

- integriert psychische und spirituelle Bedürfnisse,

- bietet Unterstützung an, damit das Leben der Patienten bis zum Tod so aktiv wie möglich sein kann,

- bietet der Familie während der Erkrankung des Patienten aber auch in der Trauerphase Unterstützung an,

- bejaht das Leben und sieht das Sterben als einen normalen Prozess an,

- will den Tod weder beschleunigen noch hinauszögern.

Diese kursorisch beschriebenen Leitsätze der Palliativmedizin können innerhalb unterschiedlicher Konzepte realisiert werden. In Deutschland haben sich ambulante Versorgungsdienste etabliert, welche - häufig durch Pflegekräfte - die Betreuung Sterbender in der häuslichen Umgebung vor allem mit schmerztherapeutischen Maßnahmen übernehmen. Leider werden diese sehr hilfreichen Dienste von den Krankenkassen bisher gar nicht oder nur sporadisch finanziert, so dass Spenden größeren Umfangs und ehrenamtliche Unterstützung Voraussetzung dafür sind. Aus leidvoller Erfahrung ist bekannt, dass die an sich sehr lobenswer-

4 WHO (1990): Cancer Pain Relief and Palliative Care. Report of a WHO Expert Committee. Technical Report Series No 804. Geneva 
ten Prinzipien des Ehrenamtes und des Sponsorings die Existenz der ambulanten Versorgung, z. B. an unter extremen Tumorschmerzen leidenden Patienten, nicht auf Dauer sichern können.

Jahr für Jahr gerät z. B. das 1996 sehr erfolgreiche, gemeinsam von der Ärztekammer Niedersachsen und unserem Zentrum durchgeführte Göttinger Projekt zur ambulanten regionalen Versorgung von Tumorschmerzpatienten in finanzielle Not. Die Krankenkassen verweigern die Übernahme in die Regelversorgung mit dem Hinweis, dass die schmerztherapeutische Versorgung der Bevölkerung im Budget der Kassenärztlichen Vereinigungen für die ambulante Patientenversorgung schon enthalten sei. Die Finanztöpfe der KV sind aber ihrerseits schon maximal ausgeschöpft.

Das Problem stellt sich dementsprechend so dar, dass es ein ambulantes palliativmedizinisches Angebot bisher flächendeckend gar nicht gibt und ohne zusätzliche Finanzierung auch nicht geben wird. Übrigens ein treffendes Beispiel für die Ignoranz unserer gesundheitspolitisch Verantwortlichen. Immerhin hat der Sachverständigenrat für die konzertierte Aktion im Gesundheitswesen konstatiert: „Die Linderung von Schmerzen stellt eine der wesentlichen ärztlichen Aufgaben als Voraussetzung für die Erhaltung der Lebensqualität dar. Das Ziel der Schmerzfreiheit bzw. der effektiven Schmerzreduktion kann bei der überwiegenden Mehrheit der Personen mit krebsbedingten Schmerzen erreicht werden. Es gibt jedoch hinreichend sichere Hinweise, dass Schmerzen bei vielen Krebskranken in Deutschland nicht ausreichend behandelt werden."

Auch in der stationären Versorgung durch ärztlich geleitete Palliativstationen, die sich von der Pflegeeinrichtung Hospiz unterscheiden, ist Deutschland im europäischen Vergleich eines der Entwicklungsländer. Mehrere Studien haben ergeben, dass der Bedarf an Palliativbetten in Deutschland um ein Vielfaches hinter dem Bedarf von etwa 30-50 Betten pro 1 Million Einwohner zurückliegt. Besserung ist nur ansatzweise in Sicht. Unter diesen Umständen einer katastrophalen palliativmedizinischen Unterversorgung mit ambulanten, stationären Strukturen und Pflegeeinrichtungen ist es nicht verwunderlich, wenn die Diskussion um die Sterbehilfe aktuell bleibt.

\section{Was kann die Palliativmedizin leisten?}

Es gilt der auf Cicely Saunders zurückgehende Grundsatz: „Nicht dem Leben mehr Tage hinzuzufügen, sondern den verbleibenden Tagen mehr Leben geben.“ Das Behandlungsziel ist Lebensqualität und nicht Lebensquantität.

Insofern kann die Palliativmedizin auch als das gegenwärtig beste Angebot der Sterbebegleitung angesehen werden. Palliativmedizin versucht all jenen Argumenten entgegenzuwirken, welche die in Umfragen Befragten veranlassten, für die aktive Sterbehilfe zu votieren. Dazu zählte insbesondere die Angst vor unerträglichen Leiden, Schmerzen, Einsamkeit, Entwürdigung und Autonomieverlust am 
Lebensende sowie auch die Sorge, Angehörigen und Behandlern zur Last zu fallen.

Die häufigsten Symptome von Patienten mit unheilbaren Erkrankungen, die in eine Palliativstation eingewiesen wurden, waren nach eigenen und anderen Untersuchungen und nach der Häufigkeit des Auftretens geordnet: allgemeine Schwäche, Schmerz, Verdauungsprobleme, Unruhe, Übelkeit, Atemnot, Anämie, Appetitlosigkeit, Gewichtsverlust und Verwirrtheit. Schmerztherapie ist also ein herausragendes therapeutisches Erfordernis. Aber auch für die Therapie der anderen genannten Symptome gibt es entsprechende gut erprobte Therapieansätze.

Wichtigste und ganzheitliche Maßnahme neben der Behandlung der genannten Symptome ist aber die Betreuung des Patienten mit seinen vielfältigen psychosozialen und spirituellen Problemen, die z. B. für die zahlreich erforderlichen Gespräche einen hohen Personaleinsatz, insbesondere im Pflegebereich, erfordern. Vorrangiges Ziel der Palliativmedizin ist es, dem Patienten eine gute Lebensqualität und schließlich auch das Sterben zu Hause in seiner familiären Umgebung zu ermöglichen. Palliativstationen und Hospize sind dabei insofern behilflich, als sie im Falle eines Therapieversagens oder einer Konfliktsituation als Zufluchtsort jederzeit kontaktiert werden können, manchmal auch, um hier in vertrauter Umgebung zu sterben. Vorrangiges Ziel bleibt es aber, eine qualitativ gesicherte ambulante palliativmedizinische Versorgung zu etablieren, die ein würdiges, symptomarmes und betreutes Sterben im Kreis der Angehörigen und in vertrauter häuslicher Umgebung ermöglicht.

In der nunmehr länger als 15 Jahre bestehenden Palliativstation Göttingen wurden über 1500 Patienten betreut. Nachdem diese sich oft in einem desolaten Aufnahmezustand befanden, wurden die bereits beschriebenen allgemeinen und symptomatischen Maßnahmen eingeleitet. Die Frage nach aktiver Sterbehilfe wurde danach praktisch nicht mehr gestellt, von wenigen Ausnahmen abgesehen, bei denen Patienten trotz Betreuung und Schmerzarmut aus Gründen einer allgemeinen Lebensmüdigkeit den Wunsch nach Beendigung ihres Lebens äußerten. Ähnliche Erfahrungen wurden im ambulanten Göttinger SUPPORT-Projekt gesammelt.

Auch hier war der Wunsch nach Beendigung des Lebens durch aktive Maßnahmen eine absolute Rarität. Wie weit schon heute Sterbehilfe auf einer Palliativstation gehen kann, wird im Beitrag von Müller-Busch in diesem Buch über die terminale (palliative) Sedierung dargelegt.

\section{Entwicklung der Palliativmedizin in Deutschland}

Palliativmedizin ist die Antwort auf die nicht adäquate Versorgung am Lebensende. Es ist oft nicht der Tod selbst, sondern die Angst vor einem unerträglichen Lebensende mit andauernder Übertherapie, schlechter Symptomkontrolle, Autonomieverlust und Entwürdigung, die Pioniere wie Cicely Saunders in England 
1967 dazu bewog, das St Christopher's Hospice zu gründen. Seitdem sind in zahlreichen Ländern vergleichbare Aktivitäten entstanden. In Deutschland wurde 1986 das erste stationäre Hospiz gegründet und 1983 die erste Palliativstation an der chirurgischen Universitätsklinik Köln, der später auch die Mildred Scheel Akademie zur Aus-, Fort- und Weiterbildung in der Palliativmedizin angeschlossen wurde. Abweichend vom britischen Vorbild sind die Hospizbewegung und die Entwicklung der Palliativmedizin, die zwar inhaltlich verbunden und dem gleichen Ethos verpflichtet sind, unterschiedliche Wege gegangen. Hospize, national durch die Bundesarbeitsgemeinschaft Hospiz (BAGH) vertreten, sind Pflegeeinrichtungen und werden medizinisch durch niedergelassene Ärzte versorgt. Ehrenamtliche Tätigkeiten spielen eine herausragende Rolle.

Merkmale eines Hospizes:

- Ziel ist die Versorgung mit Verbesserung der Lebensqualität.

- Voraussetzung für die Aufnahme ist, dass die ambulante Versorgung nicht möglich, die stationäre Krankenhausaufnahme nicht nötig ist.

- Ein Hospiz ist organisatorisch, wirtschaftlich und baulich selbstständig.

- Die medizinische Versorgung geschieht durch den Hausarzt.

- Es liegt eine Mischfinanzierung vor.

- Entlassung oder Betreuung der Patienten in der Terminalphase.

Eine Palliativstation wird nach folgenden Kriterien betrieben:

- Ziel ist die aktive, palliativmedizinische Behandlung.

- Voraussetzung für die Aufnahme ist die Krankenhausbehandlungsbedürftigkeit.

- Die Palliativstation ist eine eigenständige Krankenhausstation.

- Sie steht unter ärztlicher Leitung.

- Die Liegedauer ist begrenzt.

- Die Finanzierung erfolgt durch die Krankenkassen.

Hingegen findet die Palliativmedizin im ambulanten und vor allem stationären Bereich als Teil der ärztlich geleiteten und verantworteten medizinischen Versorgung statt. Palliativmedizin wird durch die der Arbeitsgemeinschaft der Wissenschaftlichen Medizinischen Fachgesellschaften (AWMF) angehörenden Deutschen Gesellschaft für Palliativmedizin repräsentiert (DGP). Die DGP ist multidisziplinär ausgerichtet. Ihr gehören alle Berufsgruppen einschließlich der Palliativpflege an, die in der Palliativmedizin tätig sind. Mit den gegenwärtig existierenden 130 stationären Hospizen und 115 Palliativstationen wird etwa die Hälfte des Bedarfs gedeckt. 


\section{Bedarf und Defizite in der palliativmedizinischen Versorgung in Deutschland}

Aus einer Überschlagsrechnung von Klaschik ${ }^{5}$ ergibt sich, dass: 350.000 maligne Neuerkrankungen/Jahr erwartet werden können. Davon werden primär $55 \%$ einer kurativen und $45 \%$ einer palliativen Versorgung zugeführt. Geht man bei den kurativ behandelten Patienten von einer 15\%igen Rezidivrate aus, so verbleiben $40 \%$ geheilte und $60 \%=210.000$ palliativ zu versorgende Patienten pro Jahr. Unter der Annahme, dass $10 \%$ dieser 210.000 Patienten im letzten Jahr ihrer Erkrankung ein Palliativbett benötigen und die durchschnittliche Liegezeit 13 Tage beträgt, so ergibt sich bei einer 80\%igen Stationsauslastung ein Bedarf von 33 Palliativbetten/1 Million Einwohner. Die vorhandene Zahl an Palliativbetten dürfte hingegen weniger als die Hälfte des Bedarfs decken.

Im Vergleich zur stationären Versorgung sind die Defizite in der ambulanten Palliativversorgung weitaus gravierender. Das vielfach geforderte Motto ,ambulant vor stationär" wird hier in keiner Weise realisiert, eine eigenständige Finanzierung der ambulanten Palliativmedizin durch die gesetzlichen Krankenkassen ist nicht existent und wird von diesen auch abgelehnt. Bislang unüberwindliches Hindernis für die z. B. nach der Gesundheitsstrukturreform mögliche Etablierung von ambulant-stationären integrierten Versorgungsstrukturen ist $\mathrm{u}$. a. die sektorale Finanzierung des deutschen Gesundheitswesens, so dass gegenwärtig nur sehr wenige und häufig unvollständige Konzepte umgesetzt wurden. Im Jahr 2005 hat die DGP vorgeschlagen, flächendeckende „Ambulante Hospiz- und Palliativzentren (AHPZ)“ als organisatorisch und wirtschaftlich selbständige Einheiten zur Versorgung von Palliativpatienten zu schaffen, die im Sinne einer Beratungstätigkeit, spezialisierten Versorgung und auch Krisenintervention innerhalb von 30 Minuten erreichbar sind. In einer ersten Schätzung geht man für Deutschland von mehr als 300 solcher Zentren aus.

Das niedersächsische Gutachten aus dem Jahr 2004 „Palliativversorgung in Niedersachsen" ${ }^{6}$ sieht hingegen keine eigenständigen Palliativdienste oder Palliative-Care-Teams zusätzlich zu den bestehenden Leistungserbringern vor. Stattdessen werden auf Kreisebene organisatorische virtuelle Palliativstützpunkte ohne eindeutige rechtliche, organisatorische und wirtschaftliche Ausprägungen vorgeschlagen - ein wahrlich seltsamer Vorschlag ohne Chance auf Umsetzung.

\footnotetext{
5 Klaschik E (2002): Palliativmedizin - integraler Bestandteil der Hochleistungsmedizin. In: Aulbert E, Klaschik E, Kettler D (Hrsg.): Beiträge zur Palliativmedizin. Bd. 5. Schattauer, Stuttgart, S. 48-51

6 Buser K et al. (2004): Palliativversorgung in Niedersachsen - Bestandsaufnahme und Empfehlungen zur Weiterentwicklung, cdl.niedersachsen.de/blob/images/C8578778_L20.pdf (Stand: 27.09.2006)
} 


\section{Palliativmedizin in Göttingen}

Das bereits erwähnte Gutachten zur Palliativversorgung in Niedersachsen kommt in seiner Zusammenfassung zu folgender Aussage:

„Wir empfehlen nicht die Einrichtung von Palliativstationen in den Krankenhäusern. Eine Ausnahme bildet eine akademisch ausgerichtete Palliativstation im sog. PalliativZentrum an einer niedersächsischen Universitätsklinik. Auf Grund der dort vorhandenen umfangreichen national und international renommierten palliativmedizinischen Kompetenz empfeblen wir als Standort die Universitätsklinik Göttingen ".7

Eine solche Auszeichnung hat naturgemäß eine längere Vorgeschichte. Nach mehreren Projekten zur Evaluierung der regionalen Tumorschmerzversorgung wurde 1991 ein vom Bundesministerium für Gesundheit gefördertes bundesweites Projekt zur stationären Palliativmedizin begonnen, das ab 1998 als einzige derartige niedersächsische Einrichtung in die Regelversorgung übernommen wurde und bis heute in erweitertem Umfang fortbesteht.

1996 kam ein bundesweit außerordentlich beachtetes BMG-Projekt „Support“ zur ambulanten Betreuung terminaler Tumorpatienten in vier südniedersächsischen Landkreisen hinzu, das gemeinsam von der Ärztekammer Niedersachsen und dem Göttinger Anaesthesiezentrum durchgeführt wurde und zunächst 2002 mangels Weiterfinanzierung auslief. Das Projekt ist als ambulante Ergänzung zur Palliativstation wegen seiner integrativen Rolle bei der prä- und poststationären Betreuung heute ein unverzichtbarer Bestandteil der Palliativszene in Göttingen. Anzumerken bleibt aber, dass seit dem Auslaufen der Förderung von SUPPORT im Jahr 2002 dessen Finanzierung Jahr für Jahr in Frage gestellt ist und nur durch Spenden mühsam und in sehr reduzierter Form aufrechterhalten werden kann ${ }^{8}$.

Hoffnung ist aber aus anderen Gründen angebracht. Mit Hilfe der Deutschen Krebshilfe entsteht gegenwärtig im Universitätsklinikum ein stationäres Palliativzentrum, das auch Heimat des bisherigen ambulanten Supportteams wird. Zur Etablierung dieses vorbildlichen universitären Zentrums gehört auch eine eigenständige W3-Professur für Palliativmedizin, für die ein Berufungsverfahren gerade erfolgreich abgeschlossen wurde.

Erwähnt werden soll auch die besondere Förderung der universitären Palliativmedizin durch einen Göttinger Bürgerverein und eine eigens eingerichtete Stiftung Palliativmedizin, die die Finanzierung des künftigen Palliativzentrums flankierend unterstützen.

\footnotetext{
Buser K et al. (2004), vgl. Anm. 6

8 Ensink FBM, Bautz MT, Hanekop GG (2001): Optimierung der ambulanten palliativmedizinischen Betreuung terminal kranker Tumorpatienten am Beispiel SUPPORT - ethisch zu präferierende Alternative zur Forderung nach aktiver Sterbehilfe. Anästhesiol Intensivmed Notfallmed Schmerzther, 36, 530-537
} 


\section{Palliativmedizin - Antwort auf den Ruf nach aktiver Sterbehilfe?}

Wir treffen hier zwangsläufig auf das Leitthema der Ringvorlesung: Selbstbestimmung am Lebensende. Palliativmedizin ist als medizinisches Konzept in ungewöhnlichem Maße in der Diskussion. Medizinische Verbände, Ärztekammern, beide christliche Kirchen und politische Instanzen jedweder Provenienz äußern sich zur Bedeutung der Palliativmedizin - allerdings vornehmlich als willkommenes Instrument zur Abwehr der in Umfragen mehrfach befürworteten aktiven Sterbehilfe und der Beihilfe zum Suizid, die durch die Einrichtung eines Büros in Hannover der Schweizerischen Sterbehilfeorganisation Dignitas noch aktualisiert wurde.

Erfahrene Hospizler und Palliativmediziner erleben diese etwas vordergründige Diskussion durchaus mit gemischten Gefühlen und die Frage ist erlaubt, warum erst jetzt die erwähnten gesellschaftlichen Institutionen sich diesem Problem öffnen. Seit nunmehr 10 Jahren vermissen wir ein Ohr und die Entscheidungsbereitschaft bei den Krankenkassen, Sozialministerien und teilweise auch bei ärztlich-medizinischen Institutionen, sich um das Anliegen sterbenskranker Menschen effektiv zu kümmern. Stattdessen äußern sich der Bundespräsident, Bundestag, BMG, Länderparlamente, Parteien und die Kirchen nahezu täglich positiv zur Palliativmedizin ohne dass eine entsprechende Finanzierung erkennbar wird.

Schlagwortartig soll das, was gegenwärtig an hoffentlich ernst gemeinten aktuellen Verlautbarungen zur Palliativmedizin existiert, hier zitiert werden:

Enquete Kommission des Deutschen Bundestages Ethik und Recht der Modernen Medizin. Zwischenbericht „Verbesserung der Versorgung schwerstkranker und sterbender in Deutschland durch Palliativmedizin und Hospizarbeit ${ }^{\text {(9): }}$

- Empfehlung zur gesetzlichen Regelung des Anspruchs auf eine bedarfsgerechte Palliativmedizin

- Freistellung von Angehörigen für die Sterbebegleitung

- Einführung von ambulanten Palliative Care Teams

- Palliativmedizin - künftiges Pflicht- und Prüfungsfach in der ärztlichen Ausbildung und Krankenpflegeausbildung einschließlich der Altenpflegeausbildung

- Stärkung und Sicherung der Hospizarbeit

- Verbesserung der Finanzierung der ambulanten und stationären Palliativversorgung

Der Nationale Ethikrat hat in seiner gerade veröffentlichten Stellungnahme zu „Selbstbestimmung und Fürsorge am Lebensende ${ }^{\text {"10 }}$ postuliert:

9 Enquete Kommission Etbik und Recbt der Modernen Medirin (2005): Zwischenbericht „Verbesserung der Versorgung schwerstkranker und sterbender in Deutschland durch Palliativmedizin und Hospizarbeit", www.bundestag.de/parlament/gremien/kommissionen/archiv15/ethik_med/ berichte_stellg/05_06_22_zwischenbericht_palliativmedizin.pdf (Stand: 27.09.2006) 
1. Jeder unheilbar kranke und sterbende Mensch hat Anspruch darauf, unter menschenwürdigen Bedingungen behandelt, gepflegt und begleitet zu werden.

2. Bei allen Maßnahmen der Sterbebegleitung und der Therapien am Lebensende ist der Wille des Betroffenen maßgebend.

3. Jedem unheilbar kranken und sterbenden Menschen muss eine ausreichende palliativmedizinische Versorgung gewährt werden. Ärzte sollten dabei Aspekte der Lebensqualität des Patienten über solche der maximalen Verlängerung seines Lebens stellen dürfen, ohne strafrechtliche Verfolgung befürchten zu müssen.

4. Eine ausreichende stationäre und ambulante Versorgung in Pflegeheimen, Palliativstationen und Hospizen ist dringend geboten.

5. Das Angebot an interdisziplinärer Aus- und Fortbildung für Ärzte und Pflegende im Umgang mit schwer kranken und leidenden Patienten und mit Sterbenden bedarf der Verstärkung.

6. Das ehrenamtliche Engagement bei der Sterbebegleitung sollte gefördert und unterstützt werden.

7. Angehörigen sollte eine kompetente Beratung über Pflege- und Versorgungsmöglichkeiten von schwer kranken Menschen zur Verfügung stehen.

8. Es sollten arbeitsrechtliche Freistellungsansprüche eingeräumt werden, um nahe stehenden Personen die Begleitung des Sterbenden zu ermöglichen, wie es in einigen anderen europäischen Ländern bereits realisiert ist.

Insgesamt erfahren wir also für die Palliativmedizin massive Unterstützung; angesichts der bestehenden Defizite ist diese auch sehr willkommen. Und die Gesundheitsministerin Ulla Schmidt plant eine Zur-Verfügung-Stellung von etwa 250 Millionen Euro für eine ,gute und flächendeckende Palliativmedizin, ein Gebot der Humanmedizin“.

Und auf europäischer Ebene finden sich in der Empfehlung Rec (2003) 24 des Ministerkomitees des Europarates zur „Strukturierung der palliativmedizinischen und pflegerischen Versorgung ebenfalls" wegweisende Aussagen und Forderungen zur palliativmedizinischen Versorgung und zur Selbstbestimmung am Lebensende ${ }^{11}$.

Welche Motivation den zunehmenden Bemühungen und Verlautbarungen zu Gunsten der Palliativmedizin auch zu Grunde liegen mag, es bleibt zu hoffen, dass die betroffenen, oft unerträglich leidenden Patienten in naher Zukunft daraus ihren Nutzen ziehen können. Die Zeit für die effiziente Etablierung der vierten Dimension im Gesundheitsweisen, der Palliativmedizin, ist dafür reif.

\footnotetext{
10 Nationaler Ethikrat (2006): Stellungnahme „Selbstbestimmung und Fürsorge am Lebensende“, www.ethikrat.org/stellungnahmen/pdf/Stellungnahme_Sterbebegleitung.pdf (Stand: 27.09.2006)

11 Council of Europe: Empfehlung Rec (2003) 24 des Ministerkomitees an die Mitgliedsstaaten zur Strukturierung der palliativmedizinischen und -pflegerischen Versorgung. Deutsche Übersetzung und Publikation: Deutsche Gesellschaft für Palliativmedizin. Eigenverlag, Bonn
} 


\title{
Autorenverzeichnis
}

\author{
Prof. Dr. Reiner Anselm
}

Lehrstuhl für Ethik, Theologische Fakultät, Georg-August-Universität Göttingen, Platz der Göttinger Sieben 2, 37073 Göttingen

Prof. Dr. Gunnar Duttge

Zentrum für Medizinrecht und Institut für Kriminalwissenschaften, Juristische Fakultät, Georg-August-Universität Göttingen, Goßlerstraße 19, 37073 Göttingen

Prof.(em.) Dr. Hannes Friedrich

Hermann-Föge-Weg 1, 37073 Göttingen

Prof.(em.) Dr. Dr. h.c. Dietrich Kettler

Medizinische Fakultät, Georg-August-Universität Göttingen, Robert-Koch-Straße 40, 37075 Göttingen

Prof. Dr. Volker Lipp

Zentrum für Medizinrecht und Institut für Privat- und Prozessrecht, Juristische Fakultät, Platz der Göttinger Sieben 6, 37073 Göttingen

Prof. Dr. Christof Müller-Busch

Abteilung Anästhesie, Schmerztherapie und Palliativmedizin,

Gemeinschaftskrankenhaus Havelhöhe, Kladower Damm 221, 14089 Berlin

Prof. Dr. Hilmar Prange

Abteilung Neurologie, Medizinische Fakultät, Georg-August-Universität

Göttingen, Robert-Koch-Straße 40, 37075 Göttingen

Dr. Alfred Simon

Akademie für Ethik in der Medizin e.V., Humboldtallee 36, 37073 Göttingen 



\title{
Weitere im Universitätsverlag Göttingen erschienene Titel:
}

\author{
Eva Schumann \\ Dignitas - Voluntas - Vita \\ Überlegungen zur Sterbehilfe aus rechtshistorischer, \\ interdisziplinärer und rechtsvergleichender Sicht
}

\begin{abstract}
Entgegen der heute üblichen Verengung der Sterbehilfediskussion auf die „Selbstbestimmung am Lebensende" fordert Eva Schumann ein Umdenken im Umgang mit schwerkranken und sterbenden Menschen. Um deren Bedürfnissen gerecht zu werden, ist nach ihrer Auffassung ein flächendeckender Zugang zu ambulanten und stationären Palliativeinrichtungen, eine Verbesserung der Betreuung in Altenund Pflegeheimen sowie Angebote zur stärkeren Einbindung von Angehörigen in die Pflege erforderlich. Diese Forderungen stehen am Ende einer kritischen Würdigung der unter dem Begriff der Sterbehilfe diskutierten Fallgruppen der Hilfe im Sterben (indirekte Sterbehilfe) einerseits und der Hilfe zum Sterben (aktive und passive Sterbehilfe) andererseits. Unter Einbeziehung historischer, interdisziplinärer und rechtsvergleichender Aspekte wird eindringlich dargelegt, dass die formaljuristische Differenzierung zwischen den einzelnen Fallgruppen der Sterbehilfe nicht hinreichend die soziale Dimension des Sterbens berücksichtigt.
\end{abstract}

2006, DIN A 5, Softcover, 102 Seiten | 10 Euro

ISBN-10: 3-938616-49-0

ISBN-13: 978-3-938616-49-9

Volker Lipp

Patientenautonomie und Lebensschutz

Zur Diskussion um eine gesetzliche Regelung der „Sterbehilfe“

Die rechtlichen Grundsätze für die „Sterbehilfe“ sind seit langem in Wissenschaft, Praxis und Rechtsprechung umstritten. Ende 2004 haben das Bundesjustizministerium und die Enquete-Kommission „Ethik und Recht der modernen Medizin“ des Bundestags Gesetzentwürfe vorgelegt, die sich in zentralen Fragen erheblich unterscheiden. Erfolgreich wird ein Gesetz nur sein, wenn man sich zuvor Klarheit verschafft, worum es bei der „Sterbehilfe“ geht und wie die geltende Rechtslage ist. Volker Lipp untersucht deshalb eingehend das geltende Recht der „Sterbehilfe“ und setzt sich auf dieser Grundlage mit den aktuellen Gesetzgebungsvorschlägen detailliert auseinander.

2005, DIN A 5, Softcover, 92 Seiten | 11 Euro

ISBN-10: 3-930457-74-1 